\title{
18. PALEOMAGNETISM AND ACCUMULATION RATES OF SEDIMENTS AT SITES 576 AND 578, DEEP SEA DRILLING PROJECT LEG 86, WESTERN NORTH PACIFIC1
}

\author{
G. Ross Heath, Donna H. Rea, and Shaul Levi, Oregon State University ${ }^{2}$
}

\begin{abstract}
The upper sections of Deep Sea Drilling Project Sites $576\left(32^{\circ} 21.4^{\prime} \mathrm{N}, 164^{\circ} 16.5^{\prime} \mathrm{E}\right)$ and $578\left(33^{\circ} 55.6^{\prime} \mathrm{N}, 151^{\circ} 37.7^{\prime} \mathrm{E}\right)$ both have stable detrital remanence that can be correlated with the standard reversal stratigraphy. Site 576 contains all reversals above the base of the Gilbert Epoch ( $5 \mathrm{~m} . \mathrm{y}$.) at about $25 \mathrm{~m}$, whereas Site 578 contains a remarkable section of about 60 reversals extending to Anomaly $5 \mathrm{~B}(15 \mathrm{~m} . \mathrm{y}$.) at about $150 \mathrm{~m}$ sub-bottom depth. In both cases, the paleomagnetic stratigraphy breaks down when accumulation rates drop below $2 \mathrm{~m} / \mathrm{m}$.y. At both sites, authigenic manganiferous clays deposited from 70 to $16 \mathrm{~m}$.y. ago accumulated at about $0.4 \mathrm{~m} / \mathrm{m}$.y. Similarly, at both sites, the Pleistocene pulse of eolian debris increased accumulation rates by about $6 \mathrm{~m} / \mathrm{m} . \mathrm{y} .{ }^{2}$. From 16 to $2 \mathrm{~m} . \mathrm{y}$. ago, however, sediment accumulated at Site 578 about five times as rapidly as at Site 576, apparently because of augmented input to the western site by bottom currents.
\end{abstract}

\section{INTRODUCTION}

\section{Geologic Setting}

Prior paleomagnetic studies of the sediments in the vicinity of Deep Sea Drilling Project (DSDP) Site 576, based on piston cores collected during cruise Vema-36 (Barton and Sopher, 1982), have shown that deposition has been extremely uniform (core to core variations in sedimentation rates are only about $6 \%$ over $10,000 \mathrm{~km}^{2}$ ). In contrast, Site 578 lies close to an east/west boundary across which there is a several-fold increase in sedimentation rate from south to north (Jacobi et al., this volume). Because of the stable remanence of the Vema-36 samples and the generally poor preservation of siliceous microfossils, we anticipated that dating of the Leg 86 sections would depend heavily on paleomagnetic stratigraphy. In addition, we were interested in the degree to which the paleomagnetic results from giant piston core LL44-GPC-3, taken north of Hawaii (Fig. 1; Prince et al., 1980) would resemble those at the lithologically similar Site 576 , some $40^{\circ}$ of longitude to the west.

The lithology of the Site 576 sediments is relatively simple. Above $27 \mathrm{~m}$, the section is dominated by yellowbrown pelagic clay rich in quartz (Leinen, this volume). This clay is terrigenous debris derived from Asia and carried to the site by upper atmosphere westerlies (Janecek, this volume). Similar material dominates late Cenozoic pelagic clays across the Pacific (Leinen and Heath, 1981; Moore and Heath, 1978).

The sediments between 27 and $56 \mathrm{~m}$ are very dark brown "slick" clays depleted in quartz and enriched in ferromanganese oxyhydroxides. These clays accumulated very slowly (less than $1 \mathrm{~m} / \mathrm{m}$.y.; Doyle and Riedel, this volume) and are dominated by authigenic components (silicates as well as oxyhydroxides). Below $56 \mathrm{~m}$, the clays

\footnotetext{
${ }^{1}$ Heath, G. R. Burckle, L. H., et al., Init. Repts. DSDP, 86: Washington (U.S. Govt. Printing Office)

2 Address: (Heath) College of Ocean and Fishery Sciences, University of Washington, Seattle, WA 98195; (Rea, Levi) College of Oceanography, Oregon State University, Corvallis, OR 97331 .
}

are interbedded with carbonate ooze derived either from fluctuations in the width of the Late Cretaceous equatorial carbonate zone or from turbidites originating on nearby topographic highs.

The deeper part of the section at Site 578 is comparable to Site 576. Above $77 \mathrm{~m}$, however, the section consists of gray green clay with variable amounts of biogenic silica and numerous thin layers of volcanic ash and dark greenish gray indurated clay that may be altered basic ash. These sediments are reduced (authigenic pyrite is common) and are richer in organic carbon than are the Site 576 deposits.

The yellow-brown clays analogous to the surficial deposits at Site 576 extend from 77 to $125 \mathrm{~m}$. The dark brown "slick" clays complete the section from 125 to $176 \mathrm{~m}$, terminating against chert overlain by a few silicified foraminifers. Unlike Site 576, the basal sediments are not calcareous, suggesting that at Site 578, biogenic sediments (now silicified to chert) gave way abruptly to pelagic clay.

\section{Methodology}

Magnetic samples were taken at $10-$ or $20-\mathrm{cm}$ intervals in visually undisturbed sections of core using a new sampling system designed by R. Karlin. This sampler produces strikingly less disturbance of the magnetic samples and of the surrounding sediment left behind in the core, particularly in stiff clays, than does the conventional technique of pressing $2 \times 2 \times 2 \mathrm{~cm}$ plastic boxes into the split sediment surface. Each sample was extracted from the core with a thin-walled, sharpened, $2 \times$ $2 \mathrm{~cm}$ stainless-steel tube. The bottom of the resultant square sample was trimmed of disturbed sediment and extruded into a standard $2 \times 2 \times 2 \mathrm{~cm}$ plastic cube by means of a tightly fitting plastic piston. The cube was trimmed to a flat surface and capped. Sample cubes were grouped into packages of 50 , each package being individually wrapped in Saran ${ }^{\otimes}$ wrap, then seawater-saturated paper toweling, then another layer of Saran ${ }^{\star}$ wrap to minimize water loss. Packages were shipped back to 


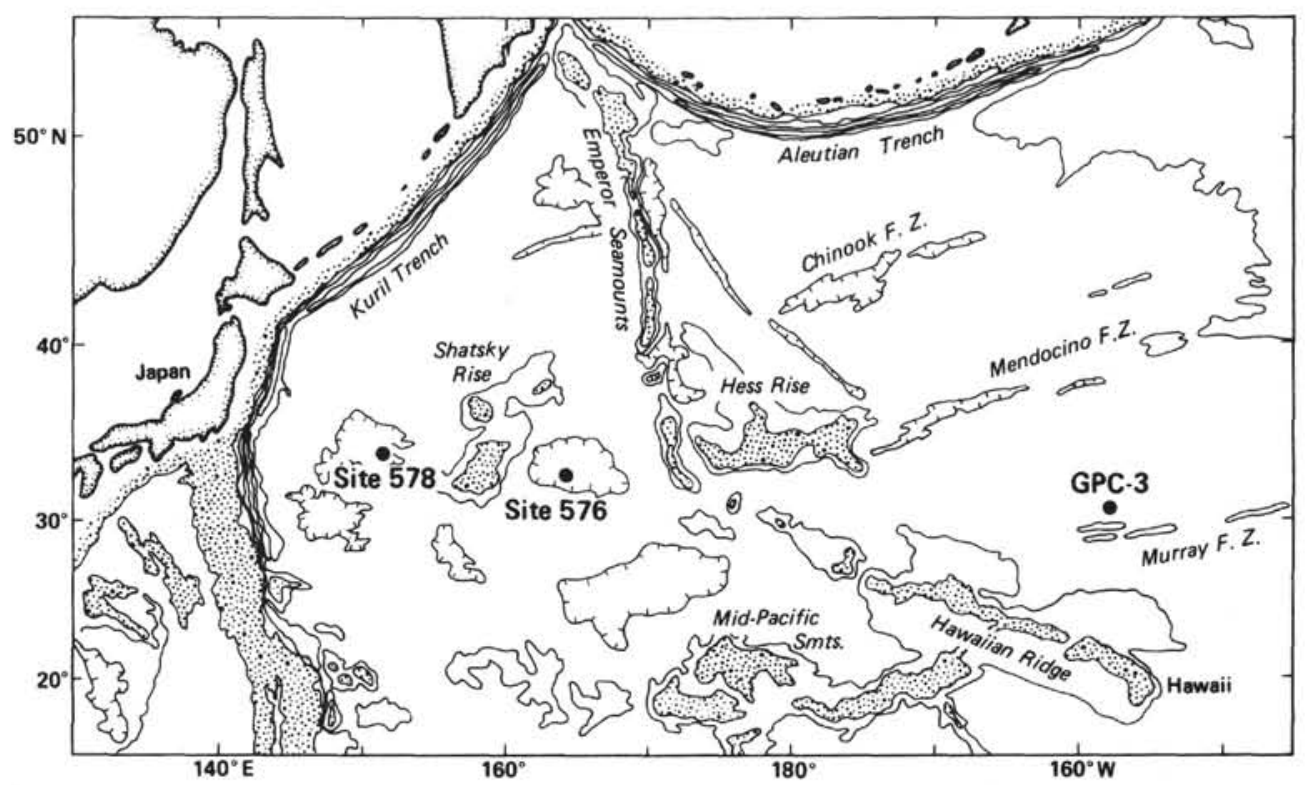

Figure 1. Location of Sites 576 and 578 and giant piston core LL44-GPC-3 relative to the generalized bathymetry of the western North Pacific (after Chase, 1975). Areas shallower than $4 \mathrm{~km}$ stippled; $5 \mathrm{~km}$ contour plain; $6 \mathrm{~km}$ contour hachured.

Oregon State University in carrying cases lined with mumetal to minimize exposure to extraneous magnetic fields.

Samples were weighed wet (for later water-content determinations) before being subjected to paleomagnetic analysis on a Digico magnetometer. Most samples were run for natural remanent magnetization (NRM), and again after alternating field (AF) demagnetization at 100 and 200 Oe. Where the remanence appeared unstable, additional measurements at higher demagnetization levels (up to $800 \mathrm{Oe}$ in some cases) were taken in an effort to characterize the original detrital remanence. The reported inclinations and declinations are based on the 100-Oe data, or on vector averages of higher demagnetization levels, depending on the behavior of intensities and directions as a function of demagnetization intensity.

\section{RESULTS}

\section{Site 576}

Tables 1 to 4 list the magnetic data for Holes 576 and 576B. Figure 2 and Table 5 show the polarity profile based on the combined results, versus the latest standard polarity scale of Berggren et al. (in press). The reversal stratigraphy is interpretable through most of the Gilbert Epoch, the base of the Thvera Event being the last reliable boundary.

Most of the reversals were picked on the basis of the inclination data, although confirmed by $180^{\circ}$ declination changes. The absolute declinations are unknown because of difficulties with the shipboard core-orienting device and rotation of the hydraulic piston core (HPC) barrel as it is driven ahead of the bit (Fig. 3).

A much more serious problem in constructing Figure 2 arose from the depth discrepancies and the condition of HPC cores from Holes 576 and 576B. The alignment of lithologic as well as magnetic boundaries suggests that Cores 576-2 and 576-3 sampled the same interval and that the top $4 \mathrm{~m}$ in Hole 576B was not recovered in Hole 576 (see Site 576 chapter, this volume). In addition, the normally magnetized intervals below the Kaena and Mammoth events are repeated in Core 576B-3, suggesting that the HPC "bounced" while sampling this interval. Such an occurrence, like the presence of "flow-in" structures in some of the cores, is indicative of significant piston motion as the HPC penetrated the sediment. We estimate that about $20 \%$ of the core recovered is so deformed as to be stratigraphically suspect. Based on the magnetic and lithologic correlations between Holes 576 and 576B, however, we believe that the composite section of Figure 2 approaches the in situ stratigraphy. There is some residual uncertainty about the thickness of the reversed section between the Olduvai Event and the base of the Matuyama Epoch (due to a small interval that lies in the gap between Cores 576-2 and 576-4 and in a disturbed section of Core 576B-2).

Based on the radiolarian stratigraphy (Morley, this volume), neither hole recovered the surficial sediments. The core-top age of 280,000 yr. (Core 576B-1), obtained by extrapolating the polarity stratigraphy (Fig. 2), agrees well with Morley's estimate of $200,000-300,000$ yr.

Below $25 \mathrm{~m}$, the reversal stratigraphy becomes uninterpretable. At this depth, the lithology changes from brown to very dark brown clay, and the sedimentation rate drops below $1 \mathrm{~m} / \mathrm{m}$.y. The average $J_{100} / J_{0}$ value also decreases abruptly (Fig. 4), suggesting that the ratio of magnetically unstable authigenic oxyhydroxides to stable detrital magnetic minerals has become so large that the detrital remanence is submerged in the "noise" of chemical remanence. A similar breakdown in stable remanence at this lithologic boundary has been recorded in other North Pacific cores (Opdyke and Foster, 1970; Kent and Lowrie, 1974; Prince et al., 1980).

The NRM profile (Fig. 4) is noisy at all depths, with a tendency to higher values below about $29 \mathrm{~m}$ and more 
Table 1. Magnetic properties of samples from Hole 576.

\begin{tabular}{|c|c|c|c|c|c|c|c|c|c|c|c|c|}
\hline 号 & ؛ั & 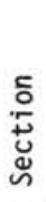 & 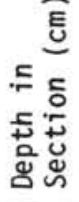 & 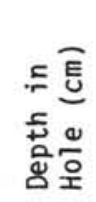 & 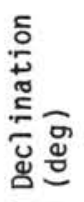 & 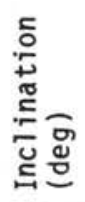 & NRM (emu) & ${ }^{J} 100$ (emu) & ${ }^{3} 100^{/ J_{0}}$ & ${ }^{J}{ }_{200}(\mathrm{emu})$ & ${ }^{3} 200^{/ J_{0}}$ & Note \\
\hline 1 & 1 & 1 & 11 & 415 & 299 & 58 & $0.4230 E-04$ & $0.3750 E-04$ & 0.887 & $0.3193 E-04$ & 0.755 & A $1-2$ \\
\hline 3 & 1 & 1 & 31 & 435 & 322 & 49 & $0.3368 E-04$ & $0.2584 E-04$ & & $0.2353 E-04$ & 0. & A $1-2$ \\
\hline 5 & 1 & 1 & 51 & 455 & 301 & 53 & $0.2309 \mathrm{E}-04$ & $0.2153 E-04$ & 32 & $0.1843 E-04$ & & A $1-2$ \\
\hline 6 & 1 & 1 & 61 & 465 & 292 & 46 & $0.3882 E-04$ & $0.2892 \mathrm{E}-04$ & & $3160 E-04$ & & A $1-4$ \\
\hline 7 & 1 & 1 & 71 & 475 & 300 & 58 & $0.4271 \mathrm{E}-04$ & $0.3785 E-04$ & 0.886 & $0.3269 E-04$ & 0.765 & A $1-2$ \\
\hline 9 & 1 & 1 & 91 & 495 & 277 & 57 & $0.3416 E-04$ & $0.2769 E-04$ & 0.811 & $0.2254 E-04$ & 0.660 & A $1-2$ \\
\hline 11 & 1 & 1 & 111 & 515 & 305 & 48 & $0.2838 E-04$ & $0.2542 E-04$ & 0.896 & $0.2078 E-04$ & 0.732 & A $1-2$ \\
\hline 13 & 1 & 1 & 131 & 535 & 300 & 56 & $0.3474 E-04$ & $0.3488 E-04$ & 1.004 & $0.2855 E-04$ & 0.822 & A $1-2$ \\
\hline 14 & 1 & 1 & 141 & 545 & 300 & 58 & $0.4269 E-04$ & $0.3895 E-04$ & 0.912 & $0.3424 E-04$ & 0.802 & A $1-2$ \\
\hline 15 & 1 & 2 & 6 & 555 & 298 & -24 & $0.5103 E-04$ & $0.4962 E-04$ & 0.972 & $0.3957 E-04$ & 0.776 & A $1-2$ \\
\hline 16 & 1 & 2 & 16 & 565 & 313 & 66 & $0.4576 E-04$ & $0.3802 E-04$ & 0.831 & $0.3414 \mathrm{E}-04$ & 0.746 & A $1-2$ \\
\hline 17 & 1 & 2 & 22 & 575 & 330 & 60 & $0.4128 E-04$ & $0.3667 E-04$ & .888 & $0.3102 E-04$ & 0.751 & A $1-2$ \\
\hline 19 & 1 & 2 & 41 & 595 & 310 & 68 & $0.3731 \mathrm{E}-04$ & $0.3434 E-04$ & 0.920 & $0.2829 E-04$ & 0.758 & A $1-2$ \\
\hline 21 & 1 & 2 & 61 & 615 & 360 & 62 & $0.3926 E-04$ & $0.3524 E-04$ & 0.897 & $E-04$ & 0.767 & A $1-2$ \\
\hline 23 & 1 & 2 & 81 & 635 & 3 & 62 & -04 & $E-04$ & 0.880 & 0.3 & & A $1-2$ \\
\hline 24 & 1 & 2 & 91 & 645 & 33 & 63 & $E-04$ & $E-04$ & 884 & 0 . & 25 & A $1-2$ \\
\hline 26 & 1 & 2 & 101 & 655 & 40 & 66 & 0 . & 0.14 & .816 & 0 & 0.686 & A $1-3$ \\
\hline 25 & 1 & * & 7 & 668 & 186 & -35 & $0.2550 E-04$ & $0.1928 E-04$ & 0.756 & $0.1476 E-04$ & 0.579 & A $1-2$ \\
\hline 27 & 2 & 1 & 6 & 701 & 263 & -54 & $0.1146 E-04$ & $0.1382 E-04$ & 1.206 & $0.1246 E-04$ & 1.088 & A $1-2$ \\
\hline 29 & 2 & 1 & 26 & 721 & 297 & -56 & $0.1599 E-04$ & $0.2161 E-04$ & 1.351 & $0.1818 E-04$ & 1.137 & A $1-2$ \\
\hline 31 & 2 & 1 & 46 & 741 & 339 & -42 & $0.2154 \mathrm{E}-04$ & $0.2321 \mathrm{E}-04$ & 1.078 & $0.1857 E-04$ & 0.862 & A $1-2$ \\
\hline 33 & 2 & 1 & 66 & 761 & 21 & -53 & $0.1397 E-04$ & $0.1635 E-04$ & 1.170 & $0.1383 E-04$ & 0.990 & A $1-2$ \\
\hline 35 & 2 & 1 & 86 & 781 & 30 & -50 & $0.1191 \mathrm{E}-04$ & $0.1252 E-04$ & 1.051 & $0.1037 E-04$ & 0.871 & A $1-2$ \\
\hline 36 & 2 & 1 & 96 & 791 & 47 & -51 & $0.2534 E-04$ & $0.2473 E-04$ & 0.976 & $0.2016 E-04$ & 0.796 & A $1-4$ \\
\hline 37 & 2 & 1 & 106 & 801 & 44 & -47 & $0.2758 E-04$ & $0.2896 \mathrm{E}-04$ & 1.050 & -04 & 0.901 & A $1-2$ \\
\hline 39 & 2 & 1 & 126 & 821 & 65 & -53 & $=-04$ & $B E-04$ & 1.129 & -04 & 27 & A \\
\hline 41 & 2 & 1 & 146 & 841 & 68 & -52 & 0 & 0 & 1.254 & 0. & & -2 \\
\hline 43 & 2 & 2 & 16 & 861 & 97 & -51 & $E-04$ & -04 & .252 & 0 & 119 & -2 \\
\hline 44 & 2 & 2 & 26 & 871 & 99 & -51 & 0.1 & $E-04$ & .026 & 0 . & 859 & A $1-2$ \\
\hline 45 & 2 & 2 & 36 & 881 & 263 & 19 & $E-$ & 05 & 263 & & 0 & L3 \\
\hline 46 & 2 & 2 & 46 & 891 & 292 & 47 & $0.1105 E-$ & 0.82 & 0.742 & 0.5 & 0.535 & -2 \\
\hline 47 & 2 & 2 & 56 & 901 & 273 & 53 & $E-$ & $B E-04$ & .838 & & 666 & \\
\hline 49 & 2 & 2 & 76 & 921 & 278 & 54 & $81 E-$ & $E-04$ & .904 & 4 & 794 & -4 \\
\hline 51 & 2 & 2 & 96 & 941 & 302 & 54 & E- & $3572 E-04$ & .893 & 0 & 1 & -2 \\
\hline 53 & 2 & 2 & 116 & 961 & 267 & -38 & $0.2869 E-05$ & $0.6663 E-06$ & .232 & 0.5 & 194 & -4 \\
\hline 55 & 2 & 2 & 136 & 981 & 143 & -57 & $0.1954 \mathrm{E}-04$ & $0.2169 E-04$ & 1.110 & -04 & 0.893 & $1-2$ \\
\hline 57 & 2 & 3 & 6 & 1001 & 163 & -53 & $0.1966 E-04$ & $0.2029 E-04$ & .032 & 0 & 868 & A $1-2$ \\
\hline 59 & 2 & 3 & 26 & 1021 & 158 & -45 & $0.2738 E-04$ & $0.2710 E-04$ & 0.990 & $0.2090 E-04$ & 0.763 & A $1-2$ \\
\hline 61 & 2 & 3 & 46 & 1041 & 172 & -52 & $0.2014 E-04$ & $0.2078 E-04$ & 1.032 & $0.1792 \mathrm{E}-04$ & 0.890 & A $1-2$ \\
\hline 63 & 2 & 3 & 66 & 1061 & 173 & -65 & $0.1663 E-04$ & $0.1853 E-04$ & 1.115 & $0.1531 E-04$ & 0.921 & A $1-2$ \\
\hline 65 & 2 & 3 & 86 & 1081 & 183 & -49 & $0.1428 E-04$ & $0.1609 E-04$ & 1.126 & $0.1311 \mathrm{E}-04$ & 0.918 & A $1-2$ \\
\hline 67 & 2 & 3 & 106 & 1101 & 174 & -50 & $0.1109 E-04$ & $0.1149 E-04$ & 1.037 & $0.1006 E-04$ & 07 & A $1-2$ \\
\hline 69 & 2 & 3 & 126 & 1121 & 171 & -49 & $0.2528 E-04$ & $0.2484 E-04$ & 0.982 & $0.2173 E-04$ & 60 & A $1-2$ \\
\hline 71 & 2 & 3 & 146 & 1141 & 196 & -59 & $0.2699 E-04$ & $0.2627 E-04$ & 0.973 & 0.2 & 58 & A $1-2$ \\
\hline 73 & 2 & 4 & 16 & 1161 & 166 & -43 & $E-04$ & $0.2224 E-04$ & 0.957 & 0 & & \\
\hline 75 & 2 & 4 & 36 & 81 & 170 & -51 & 218 & 4 & 2 & & 10 & A $1-2$ \\
\hline 77 & 2 & 4 & 56 & 1201 & 171 & -53 & 0 & & 44 & 4 & 0.875 & A $1-2$ \\
\hline 79 & 2 & 4 & 76 & 1221 & 177 & -49 & $0.2970 E-04$ & $0.2775 E-04$ & 0.934 & $0.2457 E-04$ & 0.827 & A $1-2$ \\
\hline 81 & 2 & 4 & 96 & 1241 & 180 & -56 & $0.2503 E-04$ & $0.2525 E-04$ & 1.009 & $0.2141 \mathrm{E}-04$ & 0.855 & A $1-2$ \\
\hline 83 & 2 & 4 & 116 & 1261 & 182 & -48 & $0.2596 E-04$ & $0.2720 \mathrm{E}-04$ & 1.048 & $0.1986 E-04$ & 0.765 & A $1-2$ \\
\hline 85 & 2 & 4 & 136 & 1281 & 174 & -50 & $0.1689 E-04$ & $0.1946 E-04$ & 1.152 & $0.1533 E-04$ & 0.907 & A $1-2$ \\
\hline 87 & 2 & 5 & 6 & 1301 & 172 & -46 & $0.2641 \mathrm{E}-04$ & $0.2578 E-04$ & 0.977 & $0.2275 E-04$ & 0.862 & A $1-2$ \\
\hline 89 & 2 & 5 & 26 & 1321 & 168 & -59 & $0.2302 E-04$ & $0.2427 E-04$ & 1.054 & $0.2055 E-04$ & 0.892 & A $1-2$ \\
\hline 91 & 2 & 5 & 46 & 1341 & 156 & -48 & $0.1699 E-04$ & $0.1856 E-04$ & 1.092 & $0.1477 E-04$ & 0.869 & A $1-2$ \\
\hline 93 & 2 & 5 & 66 & 1361 & 159 & -51 & $0.2477 \mathrm{E}-04$ & $0.2514 \mathrm{E}-04$ & 1.015 & $0.2028 \mathrm{E}-04$ & 0.819 & A $1-2$ \\
\hline 95 & 2 & 5 & 86 & 1381 & 158 & -59 & $0.1651 \mathrm{E}-04$ & $0.1850 E-04$ & 1.121 & $0.1448 E-04$ & 0.877 & A $1-2$ \\
\hline 96 & 2 & 5 & 96 & 1391 & 162 & -67 & $0.1218 E-04$ & $0.1443 E-04$ & 1.185 & $0.1066 \mathrm{E}-04$ & & \\
\hline 97 & 2 & 5 & 106 & 1401 & 287 & -69 & $0.7365 E-05$ & $0.1077 E-04$ & 1.463 & $0.8607 E-05$ & 1.169 & A $1-2$ \\
\hline 98 & 2 & 5 & 116 & 1411 & 289 & -71 & $0.2168 E-04$ & $0.2138 E-04$ & 0.986 & $0.1733 E-04$ & 0.799 & A $1-2$ \\
\hline 99 & 2 & 5 & 126 & 1421 & 154 & -67 & $0.2143 E-04$ & $0.2139 E-04$ & 0.998 & $0.1799 E-04$ & 0.840 & A $1-3$ \\
\hline
\end{tabular}


Table 1. (Continued).

\begin{tabular}{|c|c|c|c|c|c|c|c|c|c|c|c|c|}
\hline 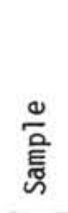 & ذँ & 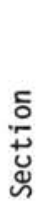 & 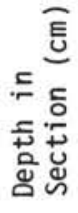 & $\begin{array}{l}\therefore \text { 틉 } \\
\text { 훙임우 }\end{array}$ & 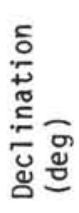 & 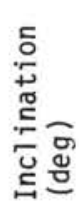 & NRM (emu) & ${ }^{3} 100(\mathrm{emu})$ & ${ }^{3} 100^{/ J_{0}}$ & ${ }^{J} 200(\mathrm{emu})$ & ${ }^{3} 200^{/ J} 0$ & Note \\
\hline 01 & 2 & 5 & 146 & 1441 & 185 & -84 & $0.2224 \mathrm{E}-04$ & $0.1966 E-04$ & 0.884 & $0.1736 E-04$ & 0.781 & 4 \\
\hline 102 & 2 & 6 & 6 & 1451 & 249 & -68 & & & 1.066 & & 882 & \\
\hline 103 & 2 & 6 & 16 & 1461 & 75 & -79 & & & 0.973 & & & \\
\hline 104 & 2 & 6 & 26 & 1 & 13 & -61 & 0 & 4 & & & 891 & \\
\hline 105 & 2 & 6 & 36 & 1481 & 254 & -43 & 0 & & & & & 2 \\
\hline 106 & 2 & 6 & 46 & 1491 & 6 & 29 & $8 E-05$ & $72 E-05$ & 547 & & 528 & \\
\hline 07 & 2 & 6 & 56 & 1501 & 343 & 38 & $0.5924 E-05$ & $729 E-05$ & 67 & $0.3919 E-05$ & 662 & A $1-2$ \\
\hline 08 & 2 & 6 & 66 & 1511 & 4 & 34 & $0.4720 E-05$ & $0.4060 E-05$ & 0.860 & $0.2946 E-05$ & 0.624 & A $1-2$ \\
\hline 09 & 2 & 6 & 76 & 1521 & 22 & 14 & $0.3792 E-05$ & $0.4242 E-05$ & & $3337 E-05$ & 880 & A $1-3$ \\
\hline 10 & 2 & 6 & 86 & 1531 & 20 & -20 & $0.1421 \mathrm{E}-04$ & $0.1262 E-04$ & 0.888 & $0.9781 E-05$ & 688 & A $1-3$ \\
\hline 111 & 2 & 6 & 96 & 1541 & 6 & 28 & $0.1448 E-04$ & $0.1215 E-04$ & 839 & $0.9639 E-05$ & 666 & A $1-3$ \\
\hline 113 & 2 & 6 & 116 & 1561 & 329 & 19 & $0.9358 E-05$ & $0.8217 E-05$ & 0.878 & 0.71 & & \\
\hline 114 & 2 & 6 & 126 & 1571 & 335 & 19 & 0 . & & & & & \\
\hline 115 & 2 & 6 & 136 & 1581 & 32 & 51 & & & & & 593 & \\
\hline 17 & 2 & 7 & 6 & 16 & 352 & 45 & & & & & 20 & -2 \\
\hline 118 & 2 & 7 & 16 & 1611 & 185 & -53 & & $E-05$ & 123 & 0 . & 994 & -2 \\
\hline 119 & 2 & 7 & 26 & 1621 & 198 & -56 & $E-05$ & $E-05$ & 0.865 & $0.3012 E-05$ & .416 & A $1-2$ \\
\hline 21 & 2 & 7 & 46 & 1641 & 153 & -24 & $4 E-05$ & $7 E-05$ & 98 & $0.2156 E-05$ & .076 & A $1-2$ \\
\hline 123 & 3 & 1 & 36 & 1021 & 288 & -58 & $0.6702 E-05$ & $0.7439 E-05$ & 1.110 & $0.6139 E-05$ & 916 & A $1-2$ \\
\hline 25 & 3 & 1 & 56 & 1041 & 285 & -49 & $0.1256 E-04$ & $0.2136 E-04$ & 1.701 & -04 & .893 & A $1-2$ \\
\hline 27 & 3 & 1 & 76 & 1061 & 291 & -45 & $E-05$ & $E-04$ & 82 & & 068 & $1-2$ \\
\hline 29 & 3 & 1 & 96 & 1081 & 281 & -62 & -05 & -05 & & & & \\
\hline 31 & 3 & 1 & 116 & 1101 & 278 & -38 & 0 . & -04 & 4 & & 1 & \\
\hline 33 & 3 & 1 & 136 & 1121 & 281 & -50 & 0. & 0. & 1 & & & \\
\hline 3 & 3 & 2 & 6 & 1141 & 311 & -46 & 0 . & 0 & 2 & & & \\
\hline 7 & 3 & 2 & 26 & 1 & 296 & -57 & & & & & & \\
\hline 39 & 3 & 2 & 46 & 1 & 296 & -52 & 0. & & & & 33 & -2 \\
\hline 41 & 3 & 2 & 66 & 1 & 2 & -48 & 0. & 0 & 5 & 04 & 304 & A $1-2$ \\
\hline 4. & 3 & 2 & 86 & 1221 & 291 & -47 & $E-04$ & $E-04$ & 32 & E -04 & 867 & -2 \\
\hline 4. & 3 & 2 & 106 & 1241 & 291 & -54 & $0.1870 E-04$ & $7 E-04$ & 1. & -04 & 845 & A $1-2$ \\
\hline 147 & 3 & 2 & 126 & 1261 & 281 & -42 & $0.1428 E-04$ & 0.143 & 003 & -04 & 1 & $1-2$ \\
\hline 149 & 3 & 2 & 146 & 1281 & 261 & -58 & $0.2827 E-04$ & $E-04$ & 906 & 0 . & 04 & -2 \\
\hline 151 & 3 & 3 & 16 & 1301 & 288 & -46 & $E-04$ & $E-04$ & 53 & 0. & 6 & \\
\hline 153 & 3 & 3 & 36 & 1321 & 276 & -48 & -04 & 0. & & & 5 & \\
\hline 5 & 3 & 3 & 56 & & 268 & -57 & 0. & & & & & \\
\hline 57 & 3 & 3 & 76 & 1. & 299 & -46 & & & & & & \\
\hline 159 & 3 & 3 & 6 & 1 & 271 & -47 & & & & & 4 & A $1-2$ \\
\hline 161 & 3 & 3 & 116 & 1401 & 275 & -50 & 0 & 0 & 5 & & 940 & A $1-2$ \\
\hline 6 & 3 & 3 & 136 & 1421 & 273 & -43 & 0 . & -04 & 44 & 4 & 929 & A $1-2$ \\
\hline 165 & 3 & 4 & 6 & 1441 & 284 & -51 & $E-04$ & $E-04$ & 34 & $E-04$ & 397 & -2 \\
\hline 167 & 3 & 4 & 26 & 1461 & 273 & -53 & $E-04$ & $E-04$ & 24 & 4 & 35 & -2 \\
\hline 169 & 3 & 4 & 56 & 1491 & 290 & -53 & $3 E-04$ & $E-04$ & 17 & 4 & 1 & -2 \\
\hline 7 & 3 & 4 & 76 & 1511 & 108 & 42 & $D E-04$ & $E-04$ & 97 & 4 & 3 & \\
\hline 17 & 3 & 4 & 6 & 1481 & 284 & -57 & $E-04$ & $E-04$ & 00 & 4 & 7 & \\
\hline 17 & 3 & 4 & 6 & 1 & 100 & 56 & 0. & $E-04$ & 821 & 4 & 523 & \\
\hline 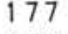 & 3 & 4 & 6 & 1 & 106 & 50 & $E-C$ & $E-04$ & & & 3 & \\
\hline 17 & 3 & 4 & 146 & 1581 & 162 & 40 & $E-05$ & $E-06$ & 4 & 6 & 5 & \\
\hline 18 & 3 & 5 & 16 & 160 & 281 & -54 & 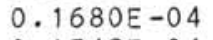 & -04 & 102 & 4 & 46 & \\
\hline 18 & 3 & * & 6 & 162 & 187 & -68 & 0. & 4 & & & 69 & \\
\hline 18 & 3 & * & 26 & 1643 & 190 & -66 & $6 E-04$ & $E-04$ & & 4 & & 2 \\
\hline 8 & 4 & 1 & 6 & & & & 0 & 5 & & & & \\
\hline 8 & 4 & 1 & 6 & & & & 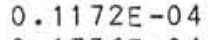 & & & & & $\mathrm{F}$ \\
\hline 18 & 4 & 1 & 26 & & & & 0 & 0 . & & & 0.514 & $\mathrm{~F}$ \\
\hline 18 & 4 & 1 & 76 & 1856 & & & $0.6859 E-05$ & $0.4927 E-05$ & 18 & $0.3259 E-05$ & 0.475 & U \\
\hline 19 & 4 & 1 & 86 & 1866 & 152 & 38 & $0.1306 E-04$ & $0.5822 E-05$ & 46 & $0.4637 E-05$ & 0.355 & A $1-2$ \\
\hline 19 & 4 & 1 & 96 & 1876 & 152 & 58 & $89 E-04$ & $0.1915 E-04$ & & $0.1481 E-04$ & 0.479 & A $1-2$ \\
\hline 19 & 4 & 1 & 106 & 1886 & 146 & 51 & $0.3843 E-04$ & $746 E-04$ & & $0.1952 E-04$ & 508 & A $1-4$ \\
\hline 19 & 4 & 1 & 116 & 1896 & 146 & 55 & $0.2768 E-$ & $053 E-04$ & & $0.1586 E-04$ & & A. 1 \\
\hline 19 & 4 & 1 & 26 & 1906 & 155 & 56 & $1100-04$ & $3500-04$ & 0.639 & $0.10100-04$ & 0.478 & A $1-2$ \\
\hline 195 & 4 & 1 & 136 & 916 & 143 & 54 & BE- & E- & & 7100 & 0.488 & A $1-2$ \\
\hline
\end{tabular}




\begin{tabular}{|c|c|c|c|c|c|c|c|c|c|c|c|c|}
\hline 咅 & ذँّ & 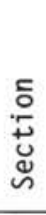 & 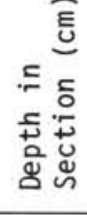 & 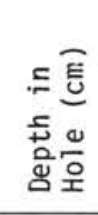 & 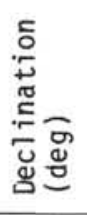 & 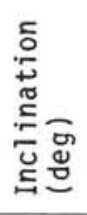 & NRM (emu) & ${ }^{J} 100(\mathrm{emu})$ & ${ }^{{ }^{3}} 100^{/ J_{0}}$ & $\mathrm{~J}_{200}(\mathrm{emu})$ & ${ }^{\mathrm{J}} 200^{/ \mathrm{J}_{0}}$ & Note \\
\hline 196 & 4 & 1 & 146 & 926 & 211 & -54 & $0.3501 E-04$ & $0.2311 \mathrm{E}-04$ & 0.660 & $0.1693 E-04$ & 0.484 & A $1-3$ \\
\hline 197 & 4 & 2 & 5 & 935 & 148 & 56 & $125 E-04$ & $284 E-04$ & & $0.1657 E-04$ & 0.530 & \\
\hline 198 & 4 & 2 & 16 & 1946 & 151 & 55 & $050 E-04$ & $868 E-04$ & 12 & $E-04$ & 439 & \\
\hline 199 & 4 & 2 & 26 & 1956 & 154 & 52 & $E-04$ & $103 E-04$ & 03 & 0.1 & 0.417 & \\
\hline 200 & 4 & 2 & 36 & 1966 & 150 & 48 & & -04 & & 0.18 & 0.505 & \\
\hline 201 & 4 & 2 & 46 & 1976 & 149 & 55 & 0. & 4 & 10 & 0.1 & 447 & \\
\hline 202 & 4 & 2 & 56 & 1986 & 341 & -49 & 0. & 0. & 88 & 05 & 180 & \\
\hline 203 & 4 & 2 & 66 & 1996 & 324 & -39 & 0.243 & 0. & 76 & $0.7875 E-05$ & 3.233 & A $1-2$ \\
\hline 204 & 4 & 2 & 76 & 2006 & 305 & -58 & $0.6574 E-05$ & 0 & 50 & $0.1046 E-04$ & .591 & A $1-2$ \\
\hline 205 & 4 & 2 & 86 & 2016 & 157 & 47 & $0.2272 E-04$ & $1103 E-04$ & 486 & $0.6830 E-05$ & .301 & A $1-2$ \\
\hline 206 & 4 & 2 & 96 & 2026 & 151 & 54 & $0.2405 E-04$ & $0.1227 E-04$ & 0.510 & $0.8846 E-05$ & 0.368 & A $1-2$ \\
\hline 207 & 4 & 2 & 106 & 2036 & 210 & -49 & $0.2257 E-04$ & $0.1092 E-04$ & 0.484 & $0.7020 E-05$ & 0.311 & A $1-2$ \\
\hline 208 & 4 & 2 & 116 & 2046 & 343 & -44 & $9 E-05$ & -05 & & $5 E-05$ & 0.345 & A $1-2$ \\
\hline 209 & 4 & 2 & 126 & 2056 & 334 & -59 & -05 & $40 E-04$ & 2.549 & 0.79 & 1.941 & \\
\hline 210 & 4 & 2 & 136 & 2066 & 347 & -54 & -05 & $=-05$ & 0.696 & 0. & 0.598 & \\
\hline 81 & 4 & 2 & 146 & 2076 & 158 & 47 & $E-04$ & -05 & 0 & 0.2 & .152 & \\
\hline 212 & 4 & 3 & 6 & 2086 & 169 & 45 & -04 & -04 & 5 & 0. & 420 & \\
\hline 215 & 4 & 3 & 16 & 2096 & 163 & 51 & $2 E-04$ & 0 & 31 & 0. & .467 & \\
\hline 14 & 4 & 3 & 26 & 2106 & 171 & 48 & $0.3024 E-04$ & 0 . & 0.510 & 0.1 & 0.360 & \\
\hline 15 & 4 & 3 & 36 & 2116 & 165 & 54 & & $10 E-04$ & & 0 & & \\
\hline 16 & 4 & 3 & 46 & 2126 & 163 & 52 & -04 & 0 & 17 & 0. & 0.438 & \\
\hline 217 & 4 & 3 & 56 & 2136 & 187 & 61 & 0 . & 0. & 28 & 0 & .254 & \\
\hline 218 & 4 & 3 & 66 & 2146 & 348 & -47 & 0. & 0 & 285 & 0. & .977 & \\
\hline 219 & 4 & 3 & 76 & 2156 & 7 & 51 & 0. & & & 5 & .644 & -6 \\
\hline 220 & 4 & 3 & 86 & 2166 & 345 & -52 & -05 & 0 & 98 & E & 2.571 & A $1-2$ \\
\hline 221 & 4 & 3 & 96 & 2176 & 354 & -55 & 0. & 4 & 367 & $0.1260 E-04$ & 1.938 & A $1-5$ \\
\hline 222 & 4 & 3 & 106 & 2186 & 348 & -57 & 0 . & 0 & 55 & $0.1156 E-04$ & 1.998 & A $1-2$ \\
\hline 223 & 4 & 3 & 116 & 2196 & 339 & -48 & $0.3430 E-05$ & $0.1190 E-04$ & 3.465 & $0.9210 E-05$ & 2.685 & A $1-2$ \\
\hline 224 & 4 & 3 & 126 & 2206 & 353 & -54 & $0.1057 E-04$ & $0.1896 E-04$ & 1.793 & $O E-$ & 1.362 & A $1-2$ \\
\hline 225 & 4 & 3 & 136 & 2216 & 338 & -54 & -05 & -04 & 96 & $6 E-$ & 1.957 & \\
\hline 226 & 4 & 3 & 146 & 2226 & 353 & -55 & -04 & -04 & 73 & $E-04$ & 1.214 & \\
\hline 227 & 4 & 4 & 6 & 2236 & 333 & -55 & $E-05$ & $0.1127 E-04$ & 2.851 & $E-05$ & 2.286 & A $1-2$ \\
\hline 228 & 4 & 4 & 16 & 2246 & 339 & -54 & -05 & $42 E-04$ & 590 & 0.1 & 1.171 & \\
\hline 229 & 4 & 4 & 6 & 2256 & 343 & -54 & $E-05$ & $E-04$ & 392 & 0.7 & 2.787 & \\
\hline 230 & 4 & 4 & 36 & 2266 & 338 & -56 & $1 E-05$ & 0 . & 29 & 0.9 & .873 & -2 \\
\hline 231 & 4 & 4 & 6 & 2276 & 187 & 54 & $E-04$ & 0. & 92 & $E-05$ & .169 & A $1-2$ \\
\hline 232 & 4 & 4 & 56 & 2286 & 164 & 57 & -04 & 0 . & 22 & 5 & .223 & A $1-2$ \\
\hline 233 & 4 & 4 & 66 & 2296 & 314 & -54 & $0.6774 E-05$ & 0. & 56 & 5 & .613 & A $1-2$ \\
\hline 234 & 4 & 4 & 76 & 2306 & 350 & -61 & $0.7140 E-05$ & 0 . & 45 & 5 & .656 & A $1-2$ \\
\hline 235 & 4 & 4 & 86 & 2316 & 339 & -56 & 0 . & 0. & 79 & $72 \mathrm{E}-$ & .033 & A $1-2$ \\
\hline 236 & 4 & 4 & 96 & 326 & 351 & -59 & $325 E-05$ & 0. & 48 & $0.9025 E-$ & 1.695 & A $1-2$ \\
\hline 237 & 4 & 4 & 06 & 336 & 182 & 50 & $510 E-04$ & $10 E-05$ & 20 & $70 E-05$ & 0.164 & A $1-2$ \\
\hline 238 & 4 & 4 & 6 & 346 & 174 & 57 & $0.2218 E-04$ & $051 E-04$ & 74 & $4 E-05$ & 319 & A $1-2$ \\
\hline 239 & 4 & 4 & 126 & 356 & 336 & -56 & $0.3885 E-05$ & $80 E-05$ & 9 & $B E-05$ & 788 & A $1-2$ \\
\hline 240 & 4 & 4 & 136 & 366 & 354 & -57 & $.2981 E-05$ & $04 E-05$ & 3 & $E-$ & 48 & A $1-2$ \\
\hline 241 & 4 & 4 & 146 & 2376 & 308 & -4 & $E-05$ & -04 & 5 & $E-$ & 83 & L \\
\hline 242 & 4 & 5 & 6 & 2386 & 355 & -52 & $94 E-05$ & -05 & 5 & $E-$ & 177 & \\
\hline 243 & 4 & 5 & 16 & 2396 & 182 & 47 & -04 & -05 & & & 0.283 & \\
\hline 244 & 4 & 5 & 26 & 2406 & 361 & -44 & $0.6492 E-05$ & -05 & 1.084 & 5 & 840 & \\
\hline 245 & 4 & 5 & 36 & 2416 & 356 & -53 & $0.1844 E-05$ & $0.9002 E-05$ & 4.881 & $0.6547 E-05$ & .550 & A $1-2$ \\
\hline 246 & 4 & 5 & 46 & 2426 & 354 & -58 & $0.9185 E-05$ & $70 E-05$ & 0 . & $0.4489 E$ & .489 & \\
\hline 24 & 4 & 5 & 56 & 2436 & 177 & 51 & $2 E-04$ & $21 \mathrm{E}-$ & & 0.9 & .366 & A 1 \\
\hline 248 & 4 & 5 & 66 & 2446 & 175 & 57 & $0.23005-04$ & $0.1023 E-04$ & 0 . & $0.7020 E-05$ & 0.305 & A $1-2$ \\
\hline 249 & 4 & 5 & 76 & 2456 & 174 & 52 & $0.1862 E-04$ & $0.8785 E-05$ & 0.472 & $0.5941 E-05$ & 0.319 & A $1-2$ \\
\hline 250 & 4 & 5 & 86 & 2466 & 185 & 52 & $0.2713 E-04$ & $0.1023 E-04$ & 0.377 & $0.6835 E-05$ & 0.252 & A $1-2$ \\
\hline 251 & 4 & 5 & 96 & 2476 & 338 & -68 & $0.7109 E-05$ & $0.3792 E-05$ & 0.5 & $0.2859 E-05$ & 0.402 & A $1-2$ \\
\hline 252 & 4 & 5 & 106 & 486 & 304 & -3 & $0.5865 E-05$ & $0.4772 E-05$ & 0.814 & $0.3833 E-05$ & 654 & \\
\hline 25 & 4 & 5 & & 96 & 5 & -53 & $473 E-05$ & $0.3784 E-05$ & & $0.2994 E-05$ & 462 & \\
\hline 254 & 4 & 5 & 126 & 2506 & 3 & -52 & $5017 E-05$ & $0.1350 E-04$ & 2 . & $0.9285 E-05$ & 1.851 & A $1-2$ \\
\hline 255 & 4 & 5 & 36 & 516 & 3 & -54 & $E-($ & & & $197 E-$ & 1.918 & A $1-2$ \\
\hline
\end{tabular}


Table 1. (Continued).

\begin{tabular}{|c|c|c|c|c|c|c|c|c|c|c|c|c|}
\hline 高 & ¿̀ & 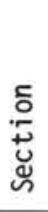 & 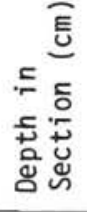 & $\begin{array}{l}\subseteq \bar{E} \\
\text { 흥 } \\
\text { 웜오 }\end{array}$ & 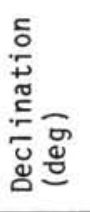 & 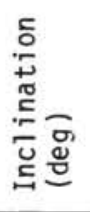 & NRN (emu) & ${ }^{J} 100(\mathrm{emu})$ & ${ }^{\mathrm{J}} 100^{/ J_{0}}$ & $\mathrm{~J}_{200}(\mathrm{emu})$ & ${ }^{\mathrm{J}} 200^{/ \mathrm{J}_{0}}$ & Note \\
\hline 256 & 4 & 5 & 146 & 2526 & 360 & -52 & $0.6473 E-05$ & $0.8143 E-05$ & 1.258 & $0.7055 E-05$ & 1.090 & A $1-2$ \\
\hline 257 & 4 & 6 & 6 & 2536 & 341 & -53 & $0.4195 E-05$ & $0.8681 E-05$ & 2.070 & $0.6251 \mathrm{E}-05$ & 1.490 & A $1-2$ \\
\hline 258 & 4 & 6 & 16 & 2546 & 349 & -53 & $0.7811 \mathrm{E}-05$ & $0.1071 E-04$ & 1.371 & $0.8373 E-05$ & 1.072 & A $1-3$ \\
\hline 259 & 4 & 6 & 26 & 2556 & 351 & -50 & $0.3809 E-05$ & $0.1025 E-04$ & 2.692 & $0.7322 \mathrm{E}-05$ & 1.922 & A $1-2$ \\
\hline 260 & 4 & 6 & 36 & 2566 & 350 & -48 & $0.7718 E-05$ & $0.1255 E-04$ & 1.626 & $0.9998 E-05$ & 1.295 & A $1-2$ \\
\hline 261 & 4 & 6 & 46 & 2576 & 333 & -21 & $0.1197 E-04$ & $0.9133 E-05$ & 0.763 & $0.6255 E-05$ & 0.523 & A $1-2$ \\
\hline 262 & 4 & 6 & 56 & 2586 & 353 & -45 & $0.10705-04$ & $0.1026 E-04$ & 0.959 & $0.7739 E-05$ & 0.723 & A $1-3$ \\
\hline 263 & 4 & 6 & 66 & 2596 & 333 & -30 & $0.1940 E-04$ & $0.4439 E-05$ & 0.229 & $0.3402 E-05$ & 0.175 & A $1-3$ \\
\hline 264 & 4 & 6 & 76 & 2606 & 340 & -20 & $0.2007 E-04$ & $0.7299 E-05$ & 0.364 & $0.5903 E-05$ & 0.294 & A $1-4$ \\
\hline 265 & 4 & 6 & 86 & 2616 & 9 & -66 & $0.1018 E-04$ & $0.1165 E-04$ & 1.145 & $0.1042 E-04$ & 1.024 & A $1-4$ \\
\hline 266 & 4 & 6 & 96 & 2626 & 335 & -45 & $0.2680 E-04$ & $0.4657 E-05$ & 0.174 & $0.3894 E-05$ & 0.145 & A 1 \\
\hline 267 & 4 & 6 & 106 & 2636 & 286 & -38 & $0.2784 E-04$ & $6 E-05$ & 0.054 & $E-05$ & 0.044 & A $2-3$ \\
\hline 268 & 4 & 6 & 116 & 2646 & 21 & -13 & $50 E-04$ & $E-05$ & 0.210 & $0.3871 E-05$ & 0.157 & A $1-4$ \\
\hline 269 & 4 & 6 & 126 & 2656 & 294 & -24 & $0.8764 E-06$ & $0.3429 E-06$ & 0.391 & $0.2684 E-06$ & 0.306 & L3 \\
\hline 270 & 4 & 6 & 136 & 2666 & 281 & -10 & $0.3355 E-04$ & $0.1105 E-05$ & 0.033 & $0.1019 E-05$ & 0.030 & L3 \\
\hline 271 & 4 & 6 & 146 & 2676 & 309 & -48 & $0.2471 E-05$ & $0.1155 E-06$ & 0.047 & $0.1431 \mathrm{E}-06$ & 0.058 & L3 \\
\hline 272 & 4 & 7 & 6 & 2686 & 282 & -7 & $0.3009 E-04$ & $0.4030 E-05$ & 0.134 & $0.2337 E-05$ & 0.078 & 13 \\
\hline 273 & 4 & 7 & 16 & 2696 & 265 & -20 & $0.1983 E-05$ & $0.3099 E-06$ & 0.156 & $0.2335 E-06$ & 0.118 & A $1-3$ \\
\hline 274 & 4 & 7 & 26 & 2706 & 357 & -40 & $0.2575 E-04$ & $0.1507 E-05$ & 0.059 & $0.1591 E-05$ & 0.062 & L3 \\
\hline 275 & 4 & 7 & 36 & 2716 & 275 & -14 & $0.1873 E-05$ & $0.2403 E-06$ & 0.128 & $0.9944 E-07$ & 0.053 & L 2.5 \\
\hline 276 & 4 & 7 & 46 & 2726 & 77 & 17 & & $0.2343 E-05$ & & $0.2260 E-05$ & & A $2-4$ \\
\hline 277 & 5 & 1 & 36 & & & & $E-04$ & $0.1094 E-04$ & 0.492 & 0.706 & 0.318 & $\mathrm{~F}$ \\
\hline 278 & 5 & 1 & 46 & 2866 & 148 & -78 & $0.9231 E-05$ & $0.8187 E-05$ & 0.887 & $0.6140 E-05$ & 0.665 & -4 \\
\hline 279 & 5 & 1 & 56 & 2876 & 243 & -66 & $0.7358 E-05$ & 0.14 & 1.988 & $0.9413 E-05$ & 1.279 & A $1-2$ \\
\hline 280 & 5 & 1 & 66 & 2886 & 220 & -69 & 0 . & $E-04$ & 1.082 & $0.7999 E-05$ & 0.763 & A $1-3$ \\
\hline 281 & 5 & 1 & 76 & 2896 & 225 & -71 & $0.5771 \mathrm{E}-05$ & $0.1108 E-04$ & 1.919 & $0.3188 E-04$ & 5.525 & -3 \\
\hline 282 & 5 & 1 & 86 & 2906 & 233 & -78 & $0.8572 E-05$ & $0.8915 E-05$ & 1.040 & $0.6218 E-05$ & 0.725 & A $1-3$ \\
\hline 283 & 5 & 1 & 96 & 2916 & 228 & -64 & $0.8385 E-05$ & $E-05$ & 1.134 & $0.6085 E-05$ & 0.726 & A $1-2$ \\
\hline 284 & 5 & 1 & 106 & 2926 & 64 & 13 & $0.1837 E-04$ & $0.4820 E-05$ & 0.262 & $0.3260 E-05$ & 0.178 & -3 \\
\hline 285 & 5 & 1 & 116 & 2936 & 71 & 6 & $0.1356 E-04$ & $E-05$ & 0.330 & 0.23 & 0.175 & A \\
\hline 286 & 5 & 1 & 126 & 2946 & 95 & -37 & $0.1402 E-04$ & $E-05$ & 0.175 & $0.1442 E-05$ & 0.103 & A $1.5-3$ \\
\hline 287 & 5 & 1 & 136 & 2956 & 87 & -4 & $E-04$ & $E-05$ & 0.223 & 0.14 & 0.133 & A $1-4$ \\
\hline 288 & 5 & 1 & 146 & 2966 & 48 & 3 & $0.1463 E-04$ & $E-05$ & 0.220 & 0.21 & 0.146 & A $1-3$ \\
\hline 289 & 5 & 2 & 56 & & & & $E-05$ & 0.5 & 1.164 & $\begin{array}{lll}0 & 31\end{array}$ & 0.718 & $F$ \\
\hline 290 & 5 & 2 & 116 & & & & $\varepsilon-$ & E- & 01 & 5 & 0.421 & F \\
\hline 291 & 5 & 3 & 23 & & & & E- & $E-$ & 68 & $E-05$ & 0.496 & $\mathrm{~F}$ \\
\hline 292 & 5 & 3 & 99 & & & & 4 & 0 . & 36 & $2296 E-05$ & 0.192 & $\mathrm{~F}$ \\
\hline 293 & 5 & 4 & 76 & & & & 5 & $E-05$ & 1.450 & $0.4248 E-05$ & 1.037 & $\mathrm{~F}$ \\
\hline 294 & 5 & 5 & 46 & & & & $0.1002 E-04$ & $235-04$ & 1.021 & $0.7204 E-05$ & 0.719 & $\mathrm{~F}$ \\
\hline 295 & 5 & 5 & 56 & 3166 & 30 & -45 & $0.1377 \subseteq-04$ & $0.1717 E-05$ & 0.125 & $0.1124 E-05$ & 0.082 & L 2 \\
\hline 296 & 5 & 5 & 66 & 3176 & 21 & 45 & $0.1507 E-04$ & $0.1925 \varepsilon-05$ & 28 & $0.6429 E-06$ & 0.043 & A $1-2$ \\
\hline 297 & 5 & 5 & 76 & 3186 & 305 & -50 & $0.1102 E-04$ & $O E-06$ & 0.086 & $0.4319 E-06$ & 0.039 & L2 \\
\hline 298 & 5 & 5 & 86 & 3196 & 352 & -53 & $0.2570 E-04$ & $4 E-05$ & 0.140 & $0.1510 E-05$ & 0.059 & L 2 \\
\hline 299 & 5 & 5 & 96 & 3206 & 360 & 52 & $0.2001 E-04$ & $0.5148 E-05$ & 0.257 & $0.3276 E-05$ & 0.164 & A $1-2$ \\
\hline 300 & 5 & 5 & 106 & 3216 & 316 & -38 & $0.1751 E-04$ & 0.12 & 0.071 & 0.428 & .024 & L 2 \\
\hline 301 & 5 & 5 & 119 & 3229 & 352 & 43 & 0.3 & 0 . & 6 & $5 E-05$ & 0.067 & A $1-2$ \\
\hline 302 & 5 & 5 & 130 & 3240 & 308 & -5 & $0.2796 \mathrm{E}-04$ & 0. & 19 & $0.5360 E-05$ & 0.192 & A $1-4$ \\
\hline 303 & 5 & 5 & 146 & 3256 & 4 & 50 & $0.4197 E-04$ & $0.9771 \mathrm{E}-05$ & 0.233 & $0.6490 E-05$ & 0.155 & A $1-2$ \\
\hline 304 & 5 & 6 & 6 & 3266 & 330 & 55 & $0.2569 E-04$ & $0.5202 E-05$ & 0.202 & $0.3078 E-05$ & 0.120 & A $1-2$ \\
\hline 305 & 5 & 6 & 16 & 3276 & 287 & 4 & $0.2975 E-04$ & $0.3134 E-05$ & 0.105 & $0.2204 E-05$ & 0.074 & L3 \\
\hline 306 & 5 & 6 & 26 & 3286 & 255 & 14 & $0.2359 E-04$ & $0.3432 E-05$ & 0.145 & $0.2357 E-05$ & 0.100 & L 3 \\
\hline 307 & 5 & 6 & 36 & 3296 & 352 & 48 & $0.3866 E-04$ & $0.8010 E-05$ & 0.207 & $0.4833 E-05$ & 0.125 & A $1-2$ \\
\hline 308 & 5 & 6 & 46 & 3306 & 305 & 30 & $0.2188 E-04$ & $0.4753 E-05$ & 0.217 & $0.2879 E-05$ & 0.132 & L 2 \\
\hline 309 & 5 & 6 & 56 & 3316 & 315 & -14 & $0.2352 E-04$ & $0.3845 E-05$ & 0.1 .63 & $0.2694 E-05$ & 0.115 & A 3-4 \\
\hline 310 & 5 & 6 & 66 & 3326 & 336 & 36 & $0.1820 E-04$ & $0.4230 E-05$ & 0.230 & $0.2320 E-05$ & 0.127 & A $1-2$ \\
\hline 311 & 5 & 6 & 76 & 3336 & 294 & -22 & $0.2021 E-04$ & $0.2613 E-05$ & 0.129 & $0.2189 E-05$ & 0.108 & L3 \\
\hline 312 & 5 & 6 & 86 & 3346 & 293 & -9 & $0.1467 E-04$ & $0.1967 E-05$ & 0.134 & $0.1028 E-05$ & 0.070 & L3 \\
\hline 313 & 5 & 6 & 96 & 3356 & 301 & 26 & $0.2851 \mathrm{E}-04$ & $0.3488 E-05$ & 0.122 & $0.19935-05$ & 0.070 & L3 \\
\hline 314 & 5 & 6 & 107 & 3367 & 265 & -9 & $0.1136 E-04$ & $0.1953 E-05$ & 0.172 & $0.1395 E-05$ & 0.123 & A $3-4$ \\
\hline 315 & 5 & 6 & 116 & 3376 & 303 & 21 & $0.2381 E-04$ & $0.3597 E-05$ & 0.151 & $0.2165 E-05$ & 0.091 & L3 \\
\hline
\end{tabular}


Table 1. (Continued).

\begin{tabular}{|c|c|c|c|c|c|c|c|c|c|c|c|c|}
\hline 吕 & ذّ & 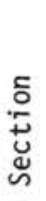 & 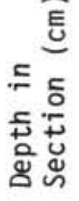 & 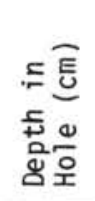 & 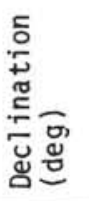 & 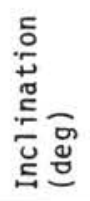 & NRM (emu) & ${ }^{J} 100(\mathrm{emu})$ & ${ }^{\mathrm{J}} 100^{/ J_{0}}$ & $\mathrm{~J}_{200}(\mathrm{emu})$ & ${ }^{J_{200}}{ }^{/ J_{0}}$ & Note \\
\hline 316 & 5 & 6 & 126 & 386 & 346 & 18 & $0.1622 E-04$ & $0.3762 E-05$ & 0.232 & $0.2371 E-05$ & 0.146 & A $2-3$ \\
\hline 317 & 5 & 6 & 135 & 995 & 339 & 39 & $0.2411 E-04$ & $.5267 E-05$ & 0.218 & $0.3423 E-05$ & 0.142 & L 2 \\
\hline 318 & 5 & 7 & 145 & 3405 & 325 & 38 & $0.1594 E-04$ & $0.4964 E-05$ & 0.311 & $0.2478 E-05$ & 0.155 & L 2 \\
\hline 319 & 5 & 7 & 6 & 3416 & 305 & 31 & $0.1947 E-04$ & $0.2338 E-05$ & 0.120 & $0.1538 E-05$ & 0.079 & L 2 \\
\hline 320 & 5 & 7 & 16 & 3426 & 345 & 39 & $0.1970 E-04$ & $0.3460 E-05$ & 0.176 & $0.1680 E-05$ & 0.085 & L 2 \\
\hline 321 & 5 & 7 & 26 & 3436 & 296 & -5 & $0.2181 E-04$ & $0.2481 E-05$ & 0.114 & $0.2162 E-05$ & 0.099 & $\llcorner 2$ \\
\hline 322 & 5 & 7 & 36 & 3446 & 342 & 55 & $0.2310 E-04$ & $0.3881 E-05$ & 0.168 & $0.1804 E-05$ & 0.078 & A $1-2$ \\
\hline 323 & 5 & 7 & 45 & 3455 & 295 & 37 & $0.2354 E-04$ & $0.1720 E-05$ & 0.073 & $0.1031 E-05$ & 0.044 & A $1-2$ \\
\hline 324 & 6 & 1 & 131 & & & & $0.3565 E-04$ & $0.3462 E-04$ & 0.971 & $0.2303 E-04$ & 0.646 & $F$ \\
\hline 325 & 6 & 1 & 141 & & & & $0.6250 E-04$ & $0.3430 E-04$ & 0.549 & $0.2305 E-04$ & 0.369 & F \\
\hline 326 & 6 & 2 & 121 & & & & $568 E-04$ & $0.1145 E-04$ & 0.321 & $.6468 E-$ & 81 & $\mathrm{~F}$ \\
\hline 327 & 6 & 2 & 131 & & & & $E-04$ & $0.7762 E-05$ & 0.170 & 0.47 & 5 & $\mathrm{~F}$ \\
\hline 328 & 6 & 2 & 141 & & & & -04 & $E-05$ & 0.332 & 0.60 & & $F$ \\
\hline 329 & 6 & 3 & 6 & & & & 0 . & 5 & 0.225 & 0.58 & 144 & n \\
\hline 330 & 6 & 3 & 26 & & & & $0.1941 E-04$ & -05 & 0.494 & 0.66 & 341 & $\mathrm{~F}$ \\
\hline 331 & 6 & 3 & 16 & & & & $0.4196 E-04$ & -04 & 0.241 & 0.64 & 154 & $r$ \\
\hline 332 & 6 & 3 & 36 & & & & $0.2396 E-04$ & $0.9235 E-05$ & 0.385 & $0.5967 E-05$ & .249 & $F$ \\
\hline 333 & 6 & 3 & 46 & & & & $0.1408 E-04$ & $0.8356 E-05$ & 0.594 & $0.5899 E-05$ & 0.419 & $\mathrm{~F}$ \\
\hline 334 & 6 & 3 & 56 & & & & $9 E-04$ & 0 & 0.455 & .64 & 0.288 & $F$ \\
\hline 335 & 6 & 3 & 66 & & & & $0.3231 E-04$ & $0.9009 E-05$ & 0.279 & $0.5245 E-05$ & 0.162 & $\mathrm{~F}$ \\
\hline 336 & 6 & 3 & 76 & 3516 & 121 & 47 & $0.2965 E-04$ & $0.9127 E-05$ & 0.308 & 0.595 & 0.201 & A $1-2$ \\
\hline 337 & 6 & 3 & 86 & 3526 & 215 & -54 & $0.2608 E-04$ & $0.8938 E-05$ & 0.343 & 0.60 & 0.233 & $1-2$ \\
\hline 338 & 6 & 3 & 96 & 3536 & 139 & 58 & $0.3078 E-04$ & $0.9072 E-05$ & 0.295 & $=-05$ & 0.234 & $1-2$ \\
\hline 339 & 6 & 3 & 106 & 3546 & 135 & 59 & 0.31 & $0.9718 E-05$ & 0.304 & 0.59 & 0.186 & $1-2$ \\
\hline 340 & 6 & 3 & 116 & 3556 & 260 & -47 & -04 & -05 & 0.090 & -05 & 0.080 & $1-4$ \\
\hline 341 & 6 & 3 & 126 & 3566 & 129 & 37 & -04 & 0.77 & 0.181 & 0.44 & 04 & $1-2$ \\
\hline 342 & 6 & 3 & 136 & 3576 & 164 & 29 & -04 & 0.858 & 0.294 & 0.54 & 0.189 & -4 \\
\hline 343 & 6 & 3 & 146 & 35 & 205 & -37 & $1 E-04$ & & & & & \\
\hline 344 & 6 & 4 & 6 & 3596 & 210 & -34 & -04 & $0.8571 E-05$ & 0.440 & 0.63 & 0.324 & $1-3$ \\
\hline 345 & 6 & 4 & 16 & 3606 & 219 & -56 & -04 & & & & & A $4-5$ \\
\hline 346 & 6 & 4 & 26 & 3616 & 212 & -52 & 0. & $0.2921 E-05$ & 0.131 & $0.2902 E-05$ & 0.130 & L 2 \\
\hline 347 & 6 & 4 & 36 & 3626 & 46 & 8 & 0 & & & & & A 5-8 \\
\hline 348 & 6 & 4 & 46 & 3636 & 65 & 45 & 0. & $0.1491 E-04$ & 0.348 & $0.1045 E-04$ & 0.244 & A $1-2$ \\
\hline 349 & 6 & 4 & 56 & 3646 & 106 & 13 & $0.5334 E-04$ & & & & & L 6 \\
\hline 350 & 6 & 4 & 66 & 3656 & 44 & 50 & $0.2894 E-04$ & $0.6069 E-05$ & 0.210 & 0.37 & 0.130 & A $1-3$ \\
\hline 351 & 6 & 4 & 76 & 3666 & 47 & 49 & $0.2629 E-04$ & & & $4 E-05$ & .244 & A $1-2$ \\
\hline 352 & 6 & 4 & 86 & 3676 & 72 & 52 & $0.1697 E-04$ & $0.4195 E-05$ & 0.247 & 0.36 & 0.213 & A $1-3$ \\
\hline 353 & 6 & 4 & 96 & 3686 & 50 & 11 & $0.2631 E-04$ & & & $0.2778 E-05$ & 0.106 & A 4-6 \\
\hline 354 & 6 & 4 & 106 & 3696 & 223 & -65 & $0.1374 E-04$ & 0.32 & 0.236 & $0.3811 E-05$ & 0.277 & A $1-2$ \\
\hline 355 & 6 & 4 & 116 & 3706 & 245 & -50 & $0.2596 E-04$ & 0.158 & 0.061 & 0.12 & 0.047 & $\llcorner 2$ \\
\hline 356 & 6 & 4 & 126 & 3716 & 54 & 20 & -04 & $0.1284 E-04$ & 0.385 & 0.57 & 0.173 & A $1-2$ \\
\hline 357 & 6 & 4 & 136 & 3726 & & & $0.2235 E-04$ & $0.7593 E-05$ & 0.340 & 0.31 & 0.140 & 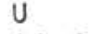 \\
\hline 358 & 6 & 4 & 146 & & 86 & 32 & -04 & 0.14 & 0.417 & 0.66 & 90 & -2 \\
\hline 359 & 6 & 5 & 6 & & 81 & 40 & -04 & $E-04$ & 0.456 & 0.89 & 69 & $1-2$ \\
\hline 360 & 6 & 5 & 16 & & 91 & 47 & -04 & $=-04$ & 0.532 & 077 & & $1-2$ \\
\hline 361 & 6 & 5 & 26 & 6 & 111 & 60 & 0 & $E-05$ & 0.757 & 0 & 55 & \\
\hline 362 & 6 & 5 & 36 & 6 & 96 & 41 & $17 E-04$ & $6 E-04$ & 0.449 & 5 & 79 & -2 \\
\hline 363 & 6 & 5 & 46 & & 93 & 57 & 0 & -05 & 0.455 & 0.44 & 299 & $1-2$ \\
\hline 364 & 6 & 5 & 56 & 6 & 73 & 64 & 0 & -05 & 0.567 & $6 E-05$ & 359 & A $1-2$ \\
\hline 365 & 6 & 5 & 66 & 6 & 88 & 62 & 0 & -05 & 43 & $0.5693 E-05$ & 0.303 & A $1-2$ \\
\hline 366 & 6 & 5 & 76 & 16 & 83 & 45 & $1874 E-04$ & $0.3066 E-05$ & 0.164 & $0.2315 E-05$ & 0.123 & A $1-2$ \\
\hline 367 & 6 & 5 & 86 & 826 & 90 & 36 & $1490 E-04$ & $0.1050 E-04$ & 0.704 & $0.7100 E-05$ & 0.476 & $1-2$ \\
\hline 368 & 6 & 5 & 96 & 836 & 109 & 14 & $1858 E-04$ & $0.3941 E-05$ & 0.212 & $0.2326 E-05$ & 0.125 & $1-2$ \\
\hline 369 & 6 & 5 & 106 & 846 & 116 & 36 & $315 E-04$ & $E-05$ & 0.406 & 0.39 & 302 & $1-2$ \\
\hline 370 & 6 & 5 & 116 & 3856 & 121 & 51 & $0.1423 E-04$ & $E-05$ & 0.529 & $0.5212 E-05$ & 0.366 & A $1-2$ \\
\hline 371 & 6 & 5 & 126 & 3866 & 123 & 44 & $0.1203 E-04$ & $0.6856 E-05$ & 0.570 & $0.4613 E-05$ & 0.384 & A $1-2$ \\
\hline 372 & 6 & 5 & 136 & 3876 & 142 & 34 & $0.2162 E-04$ & $0.4798 E-05$ & 0.222 & $0.2556 \subseteq-05$ & 0.118 & A $1-2$ \\
\hline 373 & 6 & 5 & 146 & 3886 & 125 & -13 & $0.2207 E-04$ & $0.9216 E-05$ & 0.418 & $0.15445-05$ & & -8 \\
\hline 374 & 6 & 6 & 6 & 3896 & 183 & -43 & $0.2958 E-04$ & $0.1074 E-04$ & 0.363 & $0.4622 E-05$ & 0.156 & A $1-2$ \\
\hline 375 & 6 & 6 & 16 & 3906 & 211 & 8 & $0.1659 E-04$ & $0.6864 E-05$ & 0.414 & $0.2950 E-05$ & 0.178 & A $1-2$ \\
\hline
\end{tabular}


Table 1. (Continued).

\begin{tabular}{|c|c|c|c|c|c|c|c|c|c|c|c|c|}
\hline 总 & ঠे & 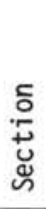 & 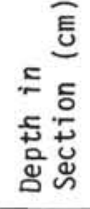 & 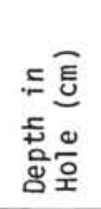 & 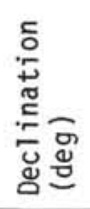 & 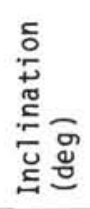 & NRM (emu) & ${ }^{J} 100(\mathrm{emu})$ & ${ }^{3} 100^{/{ }^{3}} 0$ & ${ }^{J} 200(\mathrm{emu})$ & ${ }^{\mathrm{J}} 200^{/ \mathrm{J}} 0$ & Note \\
\hline 376 & 6 & 6 & 26 & 3916 & & & $0.1122 E-04$ & & & $.2300 E-05$ & 0.205 & U \\
\hline 377 & 6 & 6 & 36 & 3926 & 183 & 33 & $0.1956 E-04$ & $0.5581 E-05$ & 0.285 & -05 & 0.122 & L3 \\
\hline 378 & 6 & 6 & 46 & 3936 & 216 & -43 & $0.3572 E-04$ & & & $1265 E-04$ & 0.354 & \\
\hline 379 & 6 & 6 & 56 & 3946 & 52 & 34 & $0.13795-04$ & $0.3150 E-05$ & 0.228 & $.3120 E-05$ & 0.226 & A $1-2$ \\
\hline 380 & 6 & 6 & 66 & 3956 & 42 & 61 & $0.2211 E-04$ & $0.7257 E-05$ & 0.328 & $E-05$ & 0.211 & $1-2$ \\
\hline 381 & 6 & 6 & 76 & 3966 & 245 & -32 & $B E-04$ & $.2535 E-05$ & 0.200 & & 0.255 & $\llcorner 2$ \\
\hline 382 & 6 & 6 & 86 & 3976 & 31 & 46 & 0 & 0,14 & .398 & & & \\
\hline 383 & 6 & 6 & 96 & 986 & 299 & 48 & 0 & 47 & 0.209 & 5 & 0.058 & L 2 \\
\hline 384 & 6 & 6 & 106 & 3996 & 290 & 27 & $0.3123 E-04$ & $0.9583 E-05$ & .307 & 5 & 0.112 & L3 \\
\hline 385 & 6 & 6 & 116 & 4006 & 30 & 11 & $0.3396 E-04$ & $0.1887 E-04$ & .556 & -04 & 0.336 & 1.2 \\
\hline 386 & 6 & 6 & 126 & 4016 & 230 & -39 & $0.1473 E-04$ & $0.2056 E-04$ & 1.396 & $435 E-04$ & 0.975 & A $1-2$ \\
\hline 387 & 6 & 6 & 136 & 4026 & 203 & -29 & $0.1920 E-04$ & $0.1439 E-04$ & 0.744 & $1043 E-04$ & 0.540 & A $1-2$ \\
\hline 388 & 6 & 6 & 146 & 4036 & 3 & 15 & $0.2742 E-04$ & $0.9641 E-05$ & 0.352 & 57 & 0.167 & A $1-3$ \\
\hline 389 & 6 & 6 & 6 & 4046 & 255 & 12 & $0.2650 E-04$ & 0.36 & 0.137 & 5 & 0.130 & L2 \\
\hline 390 & 6 & 7 & 16 & 4056 & 347 & 25 & $E-04$ & -05 & 405 & & 311 & \\
\hline 391 & 6 & 7 & 26 & 4066 & 306 & 27 & $E-04$ & -05 & 329 & 5 & 0.261 & \\
\hline 392 & 6 & 7 & 36 & 4076 & 347 & 34 & 0 & 13 & 435 & & 294 & \\
\hline 393 & 6 & 7 & 44 & 4084 & 324 & 38 & & $0.94 \quad-1$ & 303 & & 0.228 & 4 \\
\hline 394 & 7 & 1 & 9 & 4469 & & & & 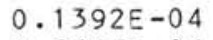 & 580 & & 089 & $\mathrm{U}$ \\
\hline 395 & 7 & 1 & 16 & 6 & 295 & -39 & & 4 & 951 & & 4 & \\
\hline 396 & 7 & 1 & 26 & 36 & 357 & -52 & & $t$ & 308 & & 4 & \\
\hline 397 & 7 & 1 & 36 & & 167 & -61 & & & 89 & & 06 & \\
\hline 398 & 7 & 1 & 46 & 6 & 211 & -45 & & 3 & 11 & & 484 & -6 \\
\hline 399 & 7 & 1 & 56 & 16 & 93 & -44 & 0 & 0.41 & 1.121 & 4 & 0.652 & $1-2$ \\
\hline 400 & 7 & 1 & 66 & 26 & 130 & -25 & $E-04$ & 0.44 & 955 & -04 & 0.552 & 3 \\
\hline 401 & 7 & 1 & 76 & 36 & 280 & 44 & $E-04$ & 0.12 & 363 & -05 & 0.225 & -2 \\
\hline 402 & 7 & 1 & 86 & 6 & 274 & 39 & & & & & 0.150 & A $1-2$ \\
\hline 403 & 7 & 1 & 96 & 56 & 270 & -49 & -04 & 5 & 01 & & & \\
\hline 404 & 7 & 1 & 106 & 4566 & 302 & 14 & $E-04$ & $E-04$ & 470 & & 4 & \\
\hline 405 & 7 & 1 & 116 & & 137 & -20 & -04 & & .509 & & 83 & \\
\hline 406 & 7 & 1 & 6 & 6 & 261 & 28 & 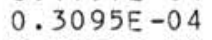 & 5 & 0 & & 9 & -2 \\
\hline 407 & 7 & 1 & 136 & 96 & 101 & -28 & 0 & $0.1298 E-05$ & .060 & & 19 & A $1-2$ \\
\hline 408 & 7 & 1 & 146 & 4606 & 283 & 1 & 0 & & & 4 & 0.407 & A $2-5$ \\
\hline 409 & 7 & 2 & 5 & & & & $=-05$ & $7 E-04$ & 2.752 & -04 & 1.903 & $\mathrm{~F}$ \\
\hline 410 & 7 & 2 & 26 & & & & $0.2066 E-04$ & & & & & F \\
\hline 411 & 7 & 2 & 36 & & & & $0.1528 \varepsilon-04$ & & & 6 & 0.059 & $F$ \\
\hline 41 & 7 & 2 & 56 & 516 & 98 & 26 & $0.2455 E-04$ & - & 1.223 & 4 & 0.570 & -2 \\
\hline 41 & 7 & $2+2+3$ & 66 & 626 & 118 & 25 & $2 E-04$ & $3141 E-04$ & 1.302 & -04 & 0.651 & -2 \\
\hline 41 & 7 & 2 & 76 & 536 & 119 & -19 & $188 E-04$ & $E-04$ & 0.819 & -04 & 98 & -2 \\
\hline 41 & 7 & 2 & 86 & 646 & 114 & -25 & $O E-$ & 42 & 1.060 & 4 & 4 & -3 \\
\hline 41 & 7 & 2 & 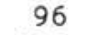 & 4656 & 111 & -38 & $E-0$ & $E-0$ & .310 & & & $1-2$ \\
\hline 41 & 7 & 2 & 108 & 6 & 272 & 57 & $t$ & 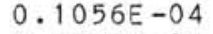 & 300 & & & -2 \\
\hline 418 & 7 & 2 & & & 290 & 33 & -04 & -04 & 468 & & & \\
\hline 41 & 7 & 2 & 126 & & 253 & 35 & 0 & $E-05$ & .215 & & & \\
\hline 42 & 7 & 2 & & & 272 & 36 & & & & & 4 & A \\
\hline 42 & 7 & 2 & 46 & & & 20 & 4 & 4 & & 5 & 0.193 & \\
\hline 422 & 7 & 3 & 5 & 6 & 258 & 23 & -04 & $E-05$ & 0.127 & -05 & 0.049 & 1 \\
\hline 42 & 7 & 3 & 14 & 6 & 116 & -32 & $=-04$ & $1113 E-04$ & 0.891 & -05 & 0.515 & A $1-2$ \\
\hline 42 & 7 & 3 & 26 & 136 & 242 & 12 & $E-04$ & $5187 E-05$ & 90 & 5 & 8 & A $2-4$ \\
\hline 42 & 7 & 3 & 36 & 746 & 159 & -23 & $756 \mathrm{~F}-0$ & $6388-05$ & 21 & -05 & 0 & -5 \\
\hline 42 & 7 & 3 & 46 & 4756 & 223 & 18 & $423 F-04$ & $2587 E-05$ & 07 & $1738 E-05$ & 72 & $4-5$ \\
\hline 42 & 7 & 3 & 56 & 766 & 286 & -23 & $g F_{-}$ & SE-05 & 0.110 & & & L5 \\
\hline 42 & 7 & 3 & 66 & 6 & 109 & -38 & $F-6+2-3$ & $E=04$ & 2.277 & & & \\
\hline 42 & 7 & 3 & 6 & 6 & 272 & 25 & $F_{-}\left(t_{1}+2\right)$ & $F-(-1)>-1$ & 35 & 5 & 0.068 & A 2 \\
\hline 43 & 7 & 3 & 86 & 6 & 89 & .33 & $F=-04$ & - & 0 & 5 & 67 & A $1-2$ \\
\hline 43 & 7 & 3 & & 806 & 99 & 46 & $F_{-}$ & $\left.F-t_{1}+2\right)$ & $0.487 \quad-$ & -05 & 0.361 & \\
\hline 43 & 7 & 3 & 06 & 4816 & 305 & -40 & $15=$ & $.6747 F-05$ & 0.297 & $4 E-05$ & 0.281 & $3-5$ \\
\hline 4 & 7 & 3 & & & & 32 & & $E-04$ & 0.387 & $1185 E-04$ & 0.263 & $1-2$ \\
\hline 43 & 7 & 3 & 126 & 4836 & 283 & 31 & $154 E-04$ & $0.2876 E-04$ & 0.402 & $0.1846 E-04$ & 0.258 & A $1-3$ \\
\hline 435 & 7 & 3 & 136 & 4846 & 277 & 34 & $0.6464 E-04$ & $0.2780 E-04$ & 0.430 & $0.1811 E-04$ & 0.280 & A $1-2$ \\
\hline
\end{tabular}


Table 1. (Continued).

\begin{tabular}{|c|c|c|c|c|c|c|c|c|c|c|c|c|}
\hline 竞 & ذั & 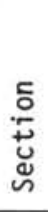 & 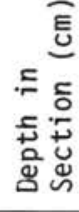 & 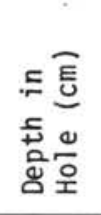 & 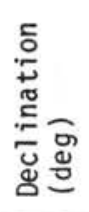 & 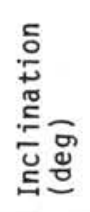 & NRM (emu) & ${ }^{\mathrm{J}} 100(\mathrm{emu})$ & ${ }^{\mathrm{J}} 100^{/ \mathrm{J}_{0}}$ & ${ }^{J_{200}}(\mathrm{emu})$ & ${ }^{J_{200}}{ }^{/ J_{0}}$ & Note \\
\hline 436 & 7 & 3 & 146 & 4856 & 275 & 36 & $0.7666 \mathrm{E}-04$ & $0.2546 E-04$ & 0.332 & $0.1650 \Xi-04$ & 0.215 & A $1-3$ \\
\hline 437 & 7 & 4 & 6 & 4866 & 272 & 30 & $0.7949 \Xi-04$ & $0.4434 \mathrm{E}-04$ & 0.558 & $0.2997 E-04$ & 0.377 & \\
\hline 438 & 7 & 4 & 16 & 4876 & 266 & 34 & $0.8817 E-04$ & $.3812 E-04$ & 0.432 & $0.2467 E-04$ & 0.280 & \\
\hline 439 & 7 & 4 & 26 & 4886 & 263 & 42 & $3 E-04$ & $1264 E-04$ & 0.277 & & 152 & \\
\hline 440 & 7 & 4 & 36 & 4896 & 278 & 40 & 0 . & $8 E-05$ & 0.073 & & 38 & \\
\hline 441 & 7 & 4 & 46 & 4906 & 270 & 36 & $E-$ & $\vec{E}-05$ & & & & \\
\hline 442 & 7 & 4 & 56 & 4916 & 251 & 49 & E- & $E-$ & 081 & & 45 & \\
\hline 443 & 7 & 4 & 66 & 4926 & 242 & 46 & $5 E-04$ & $E-05$ & 171 & & 4 & \\
\hline 444 & 7 & 4 & 76 & 4936 & 114 & -24 & $4 E-$ & E - & 518 & 5 & 07 & \\
\hline 445 & 7 & 4 & 86 & 4946 & 231 & 39 & $E-04$ & 0 & & 5 & 059 & A $1-2$ \\
\hline 446 & 7 & 4 & 96 & 4956 & 61 & -25 & 0 & 0 & 86 & 5 & 0.158 & A $2-3$ \\
\hline 447 & 7 & 4 & 106 & 4966 & 71 & -26 & $0.2411 \mathrm{E}-$ & 5 & 335 & $5789 E-05$ & 0.282 & L3 \\
\hline 448 & 7 & 4 & 116 & 4976 & 238 & 26 & $0.4212 E-04$ & 5 & 128 & $0.2747 E-05$ & 0.065 & A $1-2$ \\
\hline 449 & 7 & 4 & 126 & 4986 & 265 & 42 & $0.3993 E-04$ & $597 E-05$ & 0.190 & $0.4415 E-05$ & 0.111 & A $1-2$ \\
\hline 450 & 7 & 4 & 136 & 4996 & 62 & 17 & $0.3510 E-04$ & $0.5517 E-05$ & 0.157 & $09 E-05$ & 094 & A $1-2$ \\
\hline 451 & 7 & 4 & 146 & 5006 & 91 & -36 & 0.31 & $0.1585 E-04$ & 0.509 & -05 & 99 & \\
\hline 452 & 7 & 5 & 6 & 5016 & 76 & -6 & $0.1980 E-04$ & 0.10 & 0.510 & $=-05$ & 302 & -2 \\
\hline 453 & 7 & 5 & 16 & 5026 & 115 & -16 & 0 . & -05 & 0.135 & 0.3 & & \\
\hline 454 & 7 & 5 & 26 & 5036 & 274 & 40 & 0. & 5 & & & & \\
\hline 455 & 7 & 5 & 36 & 5046 & 260 & 51 & 0. & 4 & 226 & 0 & & \\
\hline 456 & 7 & 5 & 46 & 5056 & 255 & 24 & 0 . & 4 & & 0 & 36 & \\
\hline 457 & I & 5 & 56 & 5066 & 267 & 48 & E- & - & 0 & 5 & 6 & \\
\hline 458 & 1 & 5 & 66 & 5076 & 272 & 62 & $E-04$ & $E-$ & 77 & 5 & 09 & \\
\hline 459 & 7 & 5 & 76 & 5086 & 229 & 27 & E- & $E-$ & 45 & 5 & 97 & A3-4 \\
\hline 460 & 1 & 5 & 86 & 5096 & 242 & 34 & $E-04$ & 0 & 2 & 5 & 99 & $L$ \\
\hline 461 & 1 & 5 & 96 & 5106 & 238 & 46 & $5 E-04$ & E- & 3 & 5 & 20 & -4 \\
\hline 462 & 7 & 5 & 106 & 5116 & 76 & -40 & $5-$ & $E-$ & 223 & $E-05$ & 139 & A $1-2$ \\
\hline 463 & 7 & 5 & 116 & 5126 & 250 & 55 & $0.2939 E-04$ & $0.65665-05$ & 0.223 & $E-05$ & 170 & 2 \\
\hline 464 & 7 & 5 & 126 & 5136 & 238 & 48 & $0.4695 E-04$ & $0.7424 E-05$ & 0.158 & $0.4708 E-05$ & 0.100 & A $1-2$ \\
\hline 465 & 7 & 5 & 136 & 5146 & 79 & -18 & $0.1764 E-04$ & $0.1472 E-04$ & .834 & $E-05$ & 16 & L3 \\
\hline 466 & 7 & 5 & 146 & 5156 & 100 & -40 & $0.2715 E-04$ & $0.98095-05$ & 0.361 & $E-05$ & 70 & L \\
\hline 467 & 7 & 6 & 6 & 5166 & 258 & 70 & $0.4191 \mathrm{E}-04$ & $5377 E-05$ & 0.128 & $E-05$ & 35 & A $1-2$ \\
\hline 468 & 7 & 6 & 16 & 76 & 216 & 64 & $E-C$ & $E-05$ & .120 & -05 & 7 & \\
\hline 469 & 7 & 6 & 26 & 86 & 119 & -17 & $E-0$ & - & 4 & 5 & & \\
\hline 470 & 7 & 6 & 36 & 5196 & 268 & 55 & 0 . & -0 & 9 & 0 & & \\
\hline 471 & 7 & 6 & 46 & 5206 & 102 & 8 & 0 & -( & 7 & & & \\
\hline 472 & 7 & 6 & 56 & 6 & 94 & -28 & 0 & 4 & & & & \\
\hline 473 & 7 & 6 & 66 & 5226 & 296 & 42 & & 5 & & & & -2 \\
\hline 474 & 7 & 6 & 76 & 5236 & 83 & 26 & 0 & & & & 39 & 2 \\
\hline 475 & 7 & 6 & 86 & 5246 & & & $E-04$ & -05 & & $E-05$ & 54 & U \\
\hline 476 & 7 & 6 & 96 & 5256 & 242 & 45 & $718 E-04$ & $3 E-05$ & 48 & $3 E-05$ & 62 & -2 \\
\hline 477 & 7 & 6 & 106 & 5266 & 283 & 35 & $0.4264 E-04$ & $9500 E-05$ & 0.220 & $E-05$ & 2 & -4 \\
\hline 478 & 7 & 6 & 116 & 5276 & 284 & 33 & $0.4412 E-04$ & $1057 E-04$ & 240 & $=-05$ & 0 & A $1-2$ \\
\hline 479 & 7 & 6 & 126 & 5286 & 48 & -19 & $0.3240 E-04$ & $5910 E-05$ & 13 & -05 & 0 & 1 \\
\hline 480 & 7 & 6 & 136 & 996 & 94 & -29 & $E-04$ & -04 & 5 & 5 & & \\
\hline 481 & 7 & 6 & 146 & 6 & 108 & -15 & $E-0$ & -0 & 7 & & & A $1-4$ \\
\hline 482 & 7 & 7 & 6 & 16 & 100 & -21 & $E-04$ & $E-04$ & 5 & 4 & & L2 \\
\hline 48 & 7 & 7 & 16 & 5 & 127 & -26 & $E-04$ & $E-05$ & 1 & & & \\
\hline 4 & 7 & 7 & 6 & & 293 & 51 & $E-04$ & $=-05$ & & & & \\
\hline 485 & 7 & 7 & 36 & 5346 & 259 & 45 & -04 & -05 & & -05 & & A $2-3$ \\
\hline 486 & 7 & 7 & 46 & 5356 & 37 & -35 & $0.3210 E-04$ & $81 E-05$ & & $E-05$ & & \\
\hline 487 & 8 & 1 & 26 & 5436 & 146 & -51 & $0.2103 E-04$ & $0.2761 E-04$ & 1.313 & $E-04$ & & \\
\hline 488 & 8 & 1 & 36 & 5446 & 118 & -67 & $0.3259 E-04$ & $0.13145-04$ & 0.403 & $E-05$ & 0.300 & A $1-5$ \\
\hline & 8 & 1 & 46 & 5456 & 143 & -18 & $0.7447 E-04$ & $0.3549 E-04$ & 0.477 & $0.2502 E-04$ & 0.336 & L5 \\
\hline 490 & 8 & 1 & 56 & 5466 & 318 & -53 & $0.2850 E-04$ & $0.1308 E-04$ & 0.459 & $0.1030 E-04$ & 0.361 & A $1-5$ \\
\hline 491 & 8 & 1 & 66 & 5476 & 312 & -41 & $0.3130 E-04$ & & & $0.1094 E-04$ & 0.349 & A $1-3$ \\
\hline 92 & 8 & 1 & 76 & 5486 & 319 & -40 & $0.4522 E-04$ & $E-0$ & & $1554 E-04$ & 344 & A $1-2$ \\
\hline 03 & 8 & 1 & 86 & & 327 & .45 & $797 E-04$ & & & $1266 E-04$ & 334 & A $1-2$ \\
\hline 494 & 8 & 1 & 96 & 5506 & 190 & 1 & $0.8664 E-04$ & $0.1168 E-04$ & 0.135 & $0.5045 E-05$ & 0.058 & L 2 \\
\hline 495 & 8 & 1 & 106 & 5516 & 327 & -13 & $0.3277 E-04$ & $0.2405 E-04$ & 0.734 & $807 E-04$ & 0.552 & A $1-3$ \\
\hline
\end{tabular}


Table 1. (Continued).

\begin{tabular}{|c|c|c|c|c|c|c|c|c|c|c|c|c|}
\hline 兽 & ؛ั & 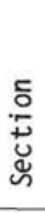 & 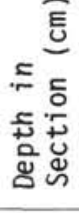 & $\begin{array}{l}\text { 드틀 } \\
\text { 등 } \\
\text { 염우 }\end{array}$ & 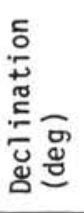 & 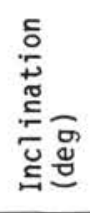 & NRM (emu) & ${ }^{J} 100(\mathrm{emu})$ & ${ }^{{ }^{3}} 100^{/ J_{0}}$ & $\mathrm{~J}_{200}$ (emu) & ${ }^{\mathrm{J}} 200^{/ \mathrm{J}} 0$ & Note \\
\hline 496 & 8 & 1 & 116 & 5526 & 328 & -34 & $0.4651 E-04$ & $0.2001 \mathrm{E}-04$ & 0.430 & $0.1615 E-04$ & 0.347 & A $1-2$ \\
\hline 497 & 8 & 1 & 26 & 5536 & 340 & -18 & $0.1956 E-04$ & $0.3853 E-04$ & 1.959 & $2857 E-$ & 1.460 & 2 \\
\hline 498 & 8 & 1 & 136 & 5546 & 190 & -3 & $0.6552 E-04$ & $0.1249 E-04$ & 0.191 & $773 E-05$ & 0.134 & A $1-3$ \\
\hline 499 & 8 & 1 & 146 & 5556 & 321 & -20 & $0.2987 E-04$ & $0.2493 E-04$ & 0.835 & $0.1628 E-04$ & 0.545 & A $1-3$ \\
\hline 500 & 8 & 2 & 5 & 5565 & 137 & 15 & $0.9755 E-04$ & $0.4520 E-04$ & 0.463 & $0.3423 E-04$ & 0.351 & A $1-4$ \\
\hline 501 & 8 & 2 & 22 & 5582 & 319 & -12 & $0.3062 E-04$ & $0.15435-04$ & 0.504 & $0.1151 E-04$ & 0.376 & A $1-2$ \\
\hline 502 & 8 & 2 & 32 & 5592 & 141 & 17 & $0.7556 E-04$ & $0.26395-04$ & 0.349 & $0.2145 E-04$ & 0.284 & A $1-4$ \\
\hline 503 & 8 & 2 & 67 & 5627 & 178 & 49 & $0.4953 E-04$ & $0.5108 E-05$ & 0.103 & $0.3312 E-05$ & 0.067 & A $1-3$ \\
\hline 504 & 8 & 2 & 78 & 5638 & 312 & -35 & $0.3043 E-04$ & $0.1294 E-04$ & 0.425 & $0.8765 E-05$ & 0.288 & A $1-2$ \\
\hline 505 & 8 & 2 & 106 & 5666 & 140 & 19 & $0.7197 E-04$ & $0.2976 E-04$ & 0.413 & $2353 \subseteq-04$ & 327 & A 1 \\
\hline 506 & 8 & 2 & 116 & 5676 & 317 & -19 & $0.1928 E-04$ & $0.3857 E-04$ & 2.000 & $0.2609 E-04$ & 1.353 & A $1-2$ \\
\hline 507 & 8 & 2 & 126 & 5686 & 292 & -9 & 0.54 & $365 E-05$ & 98 & E- & 068 & \\
\hline 508 & 8 & 2 & 136 & 96 & 313 & -17 & .04 & 4 & & 0 & 6 & -2 \\
\hline 509 & 8 & 2 & 146 & 5706 & 304 & 54 & $0.3881 E-04$ & $0.3727 E-05$ & 0.096 & 0 & 0.066 & L2 \\
\hline 510 & 8 & 3 & 4 & 14 & 132 & 21 & 0 & & & 04 & 242 & A $1-2$ \\
\hline 511 & 8 & 3 & 66 & 5776 & 123 & -20 & $0.3812 E-04$ & $6045 E-05$ & 159 & $0.2732 E-05$ & 0.072 & L3 \\
\hline 512 & 8 & 3 & 92 & 5802 & 249 & -27 & $0.5272 E-04$ & $0.9811 E-05$ & 0.186 & $0.4145 E-05$ & 0.079 & A $1-2$ \\
\hline 513 & 8 & 3 & 101 & 5811 & 146 & 18 & $0.5164 E-04$ & & & $0.3351 E-05$ & 0.065 & A $2.5-3$ \\
\hline 514 & 8 & 3 & 147 & 5857 & 311 & -14 & $0.3123 E-04$ & $0.1034 E-04$ & 0.331 & $0.94795-05$ & 0.304 & L 1 \\
\hline 515 & 8 & 4 & 3 & 5863 & 132 & -18 & $0.1948 E-04$ & $0.1337 E-04$ & 0.686 & $0.1121 E-04$ & 0.576 & L2 \\
\hline 516 & 8 & 4 & 13 & 5873 & 318 & 27 & $0.5460 E-04$ & $0.2600 E-04$ & 0.476 & $0.1740 E-04$ & 0.318 & A $1-2$ \\
\hline 517 & 8 & 4 & 23 & 5883 & 114 & 9 & $0.2491 E-04$ & $0.9294 E-05$ & 0.373 & $0.5722 \mathrm{E}-05$ & 0.230 & L 4 \\
\hline 519 & 8 & 5 & 84 & 6094 & & & $0.78160-05$ & $0.5142 E-05$ & 0.658 & $0.3232 E-05$ & 0.414 & U \\
\hline 521 & 8 & 6 & 77 & 6237 & & & & $0.5670 E-04$ & & $0.4068 E-04$ & & U \\
\hline 522 & 8 & 6 & 90 & 6250 & 345 & -14 & 0.318 & $0.4845 E-04$ & 1. & & 1.067 & A $1-4$ \\
\hline 523 & 8 & 7 & 39 & 6349 & 157 & -12 & $0.2280 E-04$ & $0.2657 E-05$ & 0.117 & $0.2048 E-05$ & 0.090 & A $2-3$ \\
\hline 524 & 8 & * & 6 & 6359 & 328 & 6 & $0.3526 E-04$ & $0.7382 E-05$ & 0.209 & $0.6771 E-05$ & 0.192 & 42 \\
\hline
\end{tabular}

Note: $A=$ vector average of declination and inclination values at demagnetization 1 evels $(x 100)$ shown.

$L=$ demagnetization level $(x, 100)$ used to define declination and inclination. $U=$ magnetically unstable sample.

$F=$ flow-in material (based on shipboard visual descriptions).

variable values below about $45 \mathrm{~m}$. In contrast, $J_{100}$ values tend to decrease downcore, but again with a marked increase in variance below about $45 \mathrm{~m}$. The $J_{100} / J_{0}$ ratio (Fig. 4) emphasizes the contrast, with a major trend from values near 1 above about $18 \mathrm{~m}$ to close to zero below about $29 \mathrm{~m}$. Demagnetization curves above (Fig. 5A, B, C) and below (Fig. 5D, E, F) the lithologic change illustrate the marked change in magnetic properties. The shallower samples show a steady decrease in intensity with increasing AF strength, with little change in the direction of magnetization. Even at $400 \mathrm{Oe}, J_{400} / J_{0}$ values exceed 0.25 . In contrast, the deeper samples show marked intensity changes (Fig. 5D) or a rapid $J / J_{0}$ decrease to values of 0.1 or less at AF strengths of $100 \mathrm{Oe}$ or more (Fig. 5E, F).

The coincidence of the transitional change in lithology, loss of paleomagnetic stratigraphy, and decrease in $J_{100}$ (both absolute and relative to NRM) point to a common sedimentological cause for these phenomena.

\section{Site 578}

Site 578 yielded an excellent magnetic record (Tables 6 and 7). Even on the Challenger, it was clear that all the magnetic events in the first four chrons were present (see Site 578 chapter, this volume; Fig. 6). Subsequent shore- based laboratory studies suggest that, with the exception of a 600,000 -yr. hiatus from 8.2 to 8.8 m.y. ago, all reversals from $15 \mathrm{~m} . \mathrm{y}$. ago to the present were recovered (Table 8). In the complex interval from the base of the Gilbert Epoch to Anomaly 5B (Fig. 7), only five samples from Site 578 (indicated by asterisks in Table 8) do not fit the standard stratigraphy.

The sequence of 60 identifiable reversals yields an extremely detailed age-depth curve (Fig. 8), the upper part of which is generally similar in form to the Site $\mathbf{5 7 6}$ curve (Fig. 2).

As at Site 576, the reversal stratigraphy is based primarily on inelination data. The declinations support these picks, but the absolute values are unknown and rotations within a single HPC can exceed $120^{\circ}$ (Fig. 9). The depth at which the reversal stratigraphy breaks down $(\sim 145 \mathrm{~m})$ again corresponds to the level at which the sedimentation rate drops below about $2 \mathrm{~m} / \mathrm{m}$.y. At Site 578 , this level lies well within a dark brown unit that is magnetically uninterpretable at Site 576, suggesting that sedimentation rate has a greater influence on the stability of detrital remanence than does gross lithology. The brown to dark brown boundary at about $125 \mathrm{~m}$ marks the point at which the sedimentation rate drops below about $4 \mathrm{~m} / \mathrm{m}$.y., but the detrital sedimentation rate was 
Table 2. Magnetic properties of samples from Hole 576B.

\begin{tabular}{|c|c|c|c|c|c|c|c|c|c|c|c|c|}
\hline 吕 & ঠั่ & 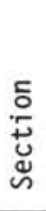 & 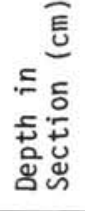 & 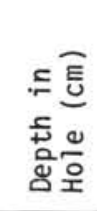 & 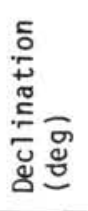 & 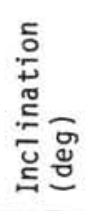 & NRM (emu) & ${ }^{J} 100$ (emu) & ${ }^{\mathrm{J}} 100^{/ \mathrm{J}_{0}}$ & $\mathrm{~J}_{200}(\mathrm{emu})$ & $\mathrm{J}_{200} / \mathrm{J}_{0}$ & Note \\
\hline 551 & 1 & 1 & 6 & 6 & 230 & 44 & $0.2352 E-04$ & $0.2050 E-04$ & 0.872 & $0.1564 E-04$ & 0.665 & L $1-2$ \\
\hline 553 & 1 & 1 & 26 & 26 & 231 & 49 & $0.36695-04$ & $0.3131 E-04$ & 0.853 & $0.2903 E-04$ & 0.791 & \\
\hline 555 & 1 & 1 & 46 & 46 & 234 & 43 & $0.3506 E-04$ & $0.26475-04$ & 0.755 & $0.2207 E-04$ & 0.630 & \\
\hline 557 & 1 & 1 & 66 & 66 & 230 & 51 & $0.3593 E-04$ & $0.3210 E-04$ & 0.893 & $0.2375 E-04$ & 0.661 & ᄂ $1-2$ \\
\hline 559 & 1 & 1 & 86 & 86 & 254 & 57 & $0.3930 E-04$ & $0.34765-04$ & 0.884 & $0.3009 E-04$ & 0.766 & L $1-2$ \\
\hline 561 & 1 & 1 & 106 & 106 & 260 & 62 & $0.3764 E-04$ & $0.3352 E-04$ & 0.891 & $0.2432 E-04$ & 0.646 & L $1-2$ \\
\hline 563 & 1 & 1 & 126 & 126 & 228 & 49 & $0.2240 E-04$ & $0.1724 E-04$ & 0.770 & $0.1362 E-04$ & 0.608 & L $1-2$ \\
\hline 565 & 1 & 2 & 146 & 146 & 265 & 50 & $0.3510 E-04$ & $0.29735-04$ & 0.847 & $0.2397 E-04$ & 0.683 & 1. $1-2$ \\
\hline 567 & 1 & 2 & 16 & 166 & 272 & 47 & $0.3218 E-04$ & $0.2940 E-04$ & 0.913 & $0.2502 E-04$ & 0.778 & $\lfloor 1-2$ \\
\hline 569 & 1 & 2 & 36 & 186 & 267 & 49 & $0.2631 E-04$ & $0.2488 E-04$ & 0.946 & $0.1917 E-04$ & 0.729 & L $1-2$ \\
\hline 571 & 1 & 2 & 56 & 206 & 260 & 54 & $0.3129 E-04$ & $0.2721 \Xi-04$ & 0.870 & $0.2439 E-04$ & 0.780 & L $1-2$ \\
\hline 573 & 1 & 2 & 76 & 226 & 270 & 45 & $0.2212 E-04$ & $0.2129 E-04$ & 0.962 & $0.1625 E-04$ & 0.735 & 11 \\
\hline 575 & 1 & 2 & 116 & 266 & 274 & 58 & $0.3380 E-04$ & $0.2795 E-04$ & 0.827 & $0.2424 E-04$ & 0.717 & -2 \\
\hline 577 & 1 & 2 & 96 & 246 & 269 & 58 & $0.2500 E-04$ & $0.2136 E-04$ & 0.854 & $3 E-04$ & 0.755 & $\lfloor 1-2$ \\
\hline 579 & 1 & 2 & 136 & 286 & 256 & 60 & $0.2554 \mathrm{E}-04$ & $0.21045-04$ & 0.824 & $35 E-$ & 0.621 & L $1-2$ \\
\hline 581 & 1 & 3 & 6 & 306 & 278 & 60 & $0.2005 E-04$ & $0.1854 E-04$ & 0.924 & $0.1460 E-04$ & 0.728 & L $1-2$ \\
\hline 583 & 1 & 3 & 26 & 326 & 293 & 62 & $0.7175 E-05$ & $0.5257 E-05$ & 0.733 & $0.4895 E-05$ & 0.682 & L $1-2$ \\
\hline 585 & 1 & 3 & 47 & 347 & 262 & 41 & $0.3212 E-04$ & $0.2195 E-04$ & 0.684 & $0.1716 E-04$ & 0.534 & L $1-2$ \\
\hline 587 & 1 & 3 & 66 & 366 & 248 & 44 & $0.2489 E-04$ & $0.2149 E-04$ & 0.863 & $0.1796 E-04$ & 0.722 & L $1-2$ \\
\hline 589 & 1 & 3 & 86 & 386 & 240 & 39 & $0.1582 E-04$ & $0.1328 E-04$ & 0.839 & $0.1101 E-04$ & 0.696 & L 1 \\
\hline 591 & 1 & 3 & 106 & 406 & 274 & 74 & $0.1839 E-04$ & $0.1492 E-04$ & 0.812 & $25-04$ & 0.708 & L $1-2$ \\
\hline 593 & 1 & 3 & 126 & 426 & 233 & 59 & $0.2469 E-04$ & $0.1791 E-04$ & 0.726 & $6 E-04$ & 0.602 & L $1-2$ \\
\hline 595 & 1 & 3 & 146 & 446 & 225 & 55 & $0.2510 E-04$ & $0.2241 E-04$ & 0.893 & $0.1856 E-04$ & 0.740 & $\lfloor 1-2$ \\
\hline 597 & 1 & 4 & 16 & 466 & 213 & 34 & $0.2199 E-04$ & $0.1767 E-04$ & 0.803 & $0.1431 E-04$ & 0.651 & $\llcorner 1-2$ \\
\hline 599 & 1 & 4 & 36 & 486 & 218 & 45 & $0.2335 E-04$ & $0.2144 \mathrm{E}-04$ & 0.918 & $0.1918 E-04$ & 0.821 & L $1-2$ \\
\hline 601 & 1 & 4 & 56 & 506 & 204 & 41 & $0.2787 E-04$ & $0.2181 E-04$ & 0.782 & $0.1887 E-04$ & 0.677 & $\llcorner 1-2$ \\
\hline 603 & 1 & 4 & 76 & 526 & 249 & 30 & $0.2734 E-04$ & $0.2301 E-04$ & 0.841 & $0.1897 E-04$ & 0.694 & ᄂ $1-2$ \\
\hline 605 & 1 & 4 & 96 & 546 & 212 & 41 & $0.2244 E-04$ & $0.1948 E-04$ & 0.868 & $0 E-04$ & 0.686 & $\llcorner 1-2$ \\
\hline 607 & 1 & 4 & 116 & 566 & 221 & 41 & $0.3107 E-04$ & $0.2849 E-04$ & 0.917 & $E-04$ & 0.753 & -2 \\
\hline 609 & 1 & 4 & 136 & 586 & 214 & 47 & $0.2882 E-04$ & $0.2566 E-04$ & 0.890 & $E-04$ & 0.704 & -2 \\
\hline 610 & 1 & 4 & 146 & 596 & 213 & 42 & $0.3409 E-04$ & $0.2821 E-04$ & 0.828 & $E-04$ & 0.647 & $\llcorner 1-2$ \\
\hline 617 & 1 & 5 & 6 & 606 & 217 & 12 & $0.2261 E-04$ & $0.1863 E-04$ & 0.824 & $E-04$ & 0.664 & $\llcorner 1-3$ \\
\hline 619 & 1 & 5 & 26 & 626 & 190 & 38 & $0.1320 E-04$ & $0.1115 E-04$ & 0.845 & $0.9069 E-05$ & 0.687 & $\llcorner 1-2$ \\
\hline 620 & 1 & 5 & 36 & 636 & 209 & -3 & $0.2120 E-04$ & $0.1424 E-04$ & 0.672 & $0.1094 E-04$ & 0.516 & $\lfloor-1-2$ \\
\hline 621 & 1 & 5 & 46 & 646 & 207 & 28 & $0.2362 E-04$ & $0.1662 E-04$ & 0.703 & $0.1419 E-04$ & 0.601 & L $1-2$ \\
\hline 622 & 1 & 5 & 56 & 656 & 201 & 25 & $0.3032 E-04$ & $0.2318 E-04$ & 0.765 & $0.2028 E-04$ & 0.669 & L $1-2$ \\
\hline 623 & 1 & 5 & 66 & 666 & 204 & -12 & $0.2282 E-04$ & $0.2111 \mathrm{E}-04$ & 0.925 & $0.1811 E-04$ & 0.794 & L $1-2$ \\
\hline 624 & 1 & 5 & 76 & 676 & 207 & -24 & $0.1960 E-04$ & $0.1571 E-04$ & 0.801 & $0.1300 E-04$ & 0.663 & $\lfloor 1-2$ \\
\hline 625 & 1 & 5 & 86 & 686 & 230 & -2 & $0.7069 E-05$ & $0.8025 E-05$ & 1.135 & $78 E-05$ & 0.832 & $\llcorner 5$ \\
\hline 626 & 1 & 5 & 96 & 696 & 276 & -45 & $0.4260 E-05$ & $0.5473 E-05$ & 1.285 & $0.5042 E-05$ & 1.184 & L $1-3$ \\
\hline 627 & 1 & 5 & 106 & 706 & 257 & -51 & $0.7136 E-05$ & $0.8119 E-05$ & 1.138 & $0.8212 E-05$ & 1.151 & $\lfloor 1-2$ \\
\hline 629 & 1 & 5 & 126 & 726 & 249 & -28 & $0.6293 E-05$ & $0.7374 \mathrm{E}-05$ & 1.172 & $0.6298 E-05$ & 1.001 & L $1-2$ \\
\hline 631 & 1 & 5 & 146 & 746 & 263 & -38 & $0.5295 E-05$ & $0.5652 E-05$ & 1.067 & $0.4454 E-05$ & 0.841 & $\lfloor 1-2$ \\
\hline 611 & 1 & 6 & 6 & 756 & 271 & -50 & $0.1245 E-04$ & $0.1510 E-04$ & 1.213 & $0.1173 E-04$ & 0.942 & $-1-3$ \\
\hline 613 & 1 & 6 & 26 & 776 & 271 & -50 & $0.1867 E-04$ & $0.1914 E-04$ & 1.025 & $0.1469 E-04$ & 0.787 & L $1-2$ \\
\hline 615 & 1 & 6 & 46 & 796 & 287 & -51 & $0.1865 E-04$ & $0.2002 E-04$ & 1.073 & $0.1543 E-04$ & 0.827 & $\llcorner 1-2$ \\
\hline 616 & 1 & 6 & 54 & 804 & 282 & -48 & $0.1572 E-04$ & $0.1477 \mathrm{E}-04$ & 0.939 & $0.1338 E-04$ & 0.851 & $\leq 1-2$ \\
\hline 632 & 2 & 1 & 11 & 171 & 27 & -31 & $0.1078 E-04$ & $0.1050 E-04$ & 0.974 & $13 E-04$ & 0.939 & L1-2, \\
\hline 633 & 2 & 1 & 19 & 1179 & 29 & -44 & $0.1477 E-04$ & $0.1693 E-04$ & 1.146 & $0.1391 E-04$ & 0.942 & เ $1-2$ \\
\hline 635 & 2 & 1 & 36 & 1196 & 34 & $-5 T$ & $0.2128 E-04$ & $0.2088 E-04$ & 0.981 & $0.1750 E-04$ & 0.822 & L $1-2$ \\
\hline 637 & 2 & 1 & 56 & 1216 & 21 & -42 & $0.2047 E-04$ & $0.2182 E-04$ & 1.066 & $0.1770 E-04$ & 0.865 & L $1-2$ \\
\hline 639 & 2 & 1 & 76 & 1236 & 24 & -56 & $0.1025 E-04$ & $0.91725-05$ & 0.895 & $0.1001 E-04$ & 0.977 & L $1-2$ \\
\hline 641 & 2 & 1 & 96 & 1256 & 29 & -50 & $0.2546 E-04$ & $0.2589 E-04$ & 1.017 & $0.1962 E-04$ & 0.770 & $L 1-2$ \\
\hline 643 & 2 & 1 & 116 & 1276 & 31 & -40 & $0.1574 E-04$ & $0.1830 E-04$ & 1.163 & $0.1358 E-04$ & 0.863 & $\llcorner 1-2$ \\
\hline 645 & 2 & 1 & 136 & 1296 & 26 & -34 & $0.1232 E-04$ & $0.1390 E-04$ & 1.128 & $0.1150 E-04$ & 0.933 & L $1-2$ \\
\hline 647 & 2 & 2 & 6 & 1316 & 19 & -31 & $0.1191 E-04$ & $0.1376 \mathrm{E}-04$ & 1.156 & $0.1191 E-04$ & 1.001 & $\llcorner 1-2$ \\
\hline 649 & 2 & 2 & 26 & 1336 & 37 & -48 & $0.9595 E-05$ & $0.1018 E-04$ & 1.061 & $0.9212 E-05$ & 0.960 & $\llcorner 1-2$ \\
\hline 651 & 2 & 2 & 46 & 1356 & 32 & -39 & $0.1742 E-04$ & $0.1991 \mathrm{E}-04$ & 1.143 & $0.1521 E-04$ & 0.873 & L $1-2$ \\
\hline 653 & 2 & 2 & 66 & 1376 & 19 & -53 & $0.1250 E-04$ & $0.1145 E-04$ & 0.916 & $0.1021 E-04$ & 0.817 & L $1-2$ \\
\hline 655 & 2 & 2 & 86 & 1396 & 27 & -48 & $0.2542 E-04$ & $0.2544 E-04$ & 1.001 & $0.2083 E-04$ & 0.819 & $\lfloor 1-2$ \\
\hline
\end{tabular}


Table 2. (Continued).

\begin{tabular}{|c|c|c|c|c|c|c|c|c|c|c|c|c|}
\hline 吕 & ذँ & 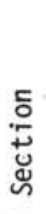 & 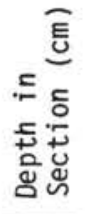 & 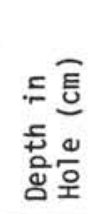 & 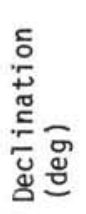 & 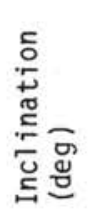 & NRN (emu) & ${ }^{J_{100}}(\mathrm{emu})$ & ${ }^{\mathrm{J}} 100^{/]_{0}}$ & $\mathrm{~J}_{200}$ (emu) & $\mathrm{J}_{200} / \mathrm{J}_{0}$ & Note \\
\hline 657 & 2 & 2 & 106 & 1416 & 25 & -49 & $0.1801 E-04$ & $0.2198 E-04$ & 1.220 & $0.1756 E-04$ & 0.975 & $\llcorner 1-2$ \\
\hline 659 & 2 & 2 & 126 & 1436 & 29 & -49 & $0.20175-04$ & $0.2135 E-04$ & 1.059 & $0.1770 E-04$ & 0.877 & L $1-2$ \\
\hline 661 & 2 & 2 & 146 & 1456 & 26 & -51 & $0.1156 E-04$ & $0.1279 E-04$ & 1.106 & $0.1156 E-04$ & 0.999 & L $1-2$ \\
\hline 663 & 2 & 3 & 15 & 1475 & 357 & -46 & $0.5785 E-05$ & $0.8371 E-05$ & 1.447 & $0.6624 E-05$ & 1.145 & L $1-2$ \\
\hline 664 & 2 & 3 & 26 & 1486 & 10 & -47 & $0.1105 E-04$ & $0.1287 E-04$ & 1.165 & $0.9473 E-05$ & 0.857 & $\lfloor 1-2$ \\
\hline 665 & 2 & 3 & 36 & 1496 & 168 & 13 & $0.8638 E-05$ & $0.4473 E-05$ & 0.518 & $0.3425 E-05$ & 396 & $\lfloor 1-2$ \\
\hline 667 & 2 & 3 & 56 & 1516 & 189 & 49 & $0.2157 E-04$ & $0.1943 E-04$ & 0.900 & $0.1406 E-04$ & 0.652 & $\lfloor 1-2$ \\
\hline 669 & 2 & 3 & 76 & 1536 & 181 & 13 & $0.2631 \mathrm{E}-04$ & $0.2115 E-04$ & 0.804 & $0.1806 E-04$ & 0.687 & $\leq 1-2$ \\
\hline 673 & 2 & 4 & 46 & 1656 & 196 & -48 & $0.2022 E-04$ & $0.2223 E-04$ & 1.099 & $0.1728 E-04$ & 0.855 & $\llcorner 1-2$ \\
\hline 674 & 2 & 4 & 56 & 1666 & 193 & -46 & $0.1308 E-04$ & $0.1492 E-04$ & 1.141 & $0.1037 E-04$ & 0.793 & L $1-2$ \\
\hline 675 & 2 & 4 & 66 & 1676 & 189 & -49 & $0.1750 E-04$ & $0.2045 E-04$ & 1.168 & $0.1697 E-04$ & 0.970 & $1-1-2$ \\
\hline 676 & 2 & 4 & 76 & 1686 & 182 & -54 & $0.1505 E-04$ & $0.1475 E-04$ & 0.981 & $0.1231 E-04$ & 0.818 & L $1-2$ \\
\hline 677 & 2 & 4 & 86 & 1696 & 184 & -41 & $0.1552 E-04$ & $0.1962 E-04$ & 1.265 & $0.1449 E-04$ & 0.934 & L $1-2$ \\
\hline 678 & 2 & 4 & 96 & 1706 & 163 & -49 & $0.2233 E-04$ & $0.2051 \leq-04$ & 0.918 & $0.1735 E-04$ & 0.777 & L 1 \\
\hline 679 & 2 & 4 & 106 & 1716 & 188 & -53 & $0.1512 E-04$ & $0.1487 E-04$ & 0.984 & $0.1183 E-04$ & 0.782 & L $1-2$ \\
\hline 680 & 2 & 4 & 116 & 1726 & 188 & -44 & $0.2453 E-04$ & $0.2465 E-04$ & 1.005 & $0.1914 \mathrm{E}-04$ & 0.780 & $\lfloor 1-2$ \\
\hline 681 & 2 & 4 & 126 & 1736 & 184 & -51 & $0.1784 E-04$ & $0.18335-04$ & 1.028 & $0.1521 E-04$ & 0.853 & $1-1-2$ \\
\hline 682 & 2 & 4 & 136 & 1746 & 194 & -68 & $0.2104 E-04$ & $0.1764 E-04$ & 0.838 & $0.1417 E-04$ & 0.674 & L $1-2$ \\
\hline 683 & 2 & 4 & 146 & 1756 & 171 & -48 & $0.1604 E-04$ & $0.1551 E-04$ & 0.967 & $0.1305 E-04$ & 0.814 & L $1-2$ \\
\hline 684 & 2 & 5 & 6 & 1766 & 164 & -68 & $0.8324 E-05$ & $0.96735-05$ & 1.162 & $0.7930 E-05$ & 0.953 & $1-1-2$ \\
\hline 685 & 2 & 5 & 16 & 1776 & 158 & -87 & $0.3707 E-05$ & $0.6104 E-05$ & 1.647 & $0.4410 E-05$ & 1.190 & L $1-3$ \\
\hline 686 & 2 & 5 & 25 & 1786 & 200 & -36 & $0.7980 E-05$ & $0.9018 E-05$ & 1.130 & $0.6499 E-05$ & 0.814 & L $1-2$ \\
\hline 687 & 2 & 5 & 36 & 1796 & 188 & -53 & $0.1391 E-04$ & $0.1539 E-04$ & 1.107 & $0.1239 E-04$ & 0.891 & ᄂ $1-2$ \\
\hline 688 & 2 & 5 & 46 & 1806 & 299 & -72 & $0.1354 E-04$ & $0.1666 E-04$ & 1.230 & $0.1155 E-04$ & 0.853 & L1 -3 \\
\hline 689 & 2 & 5 & 56 & 1816 & 298 & -71 & $0.1067 E-04$ & $0.1317 E-04$ & 1.235 & $0.1127 E-04$ & 1.056 & L $1-2$ \\
\hline 690 & 2 & 5 & 66 & 1826 & 139 & -80 & $0.1002 E-04$ & $0.1309 E-04$ & 1.306 & $0.8905 E-05$ & 0.889 & L $1-2$ \\
\hline 691 & 2 & 5 & 76 & 1836 & 131 & -63 & $0.1352 E-05$ & $0.1759 E-05$ & 1.300 & $0.1262 E-05$ & 0.933 & L $1-2$ \\
\hline 692 & 2 & 5 & 86 & 1846 & 30 & 46 & $0.1361 E-04$ & $0.9511 E-05$ & 0.699 & $0.7171 E-05$ & 0.527 & L 1 \\
\hline 693 & 2 & 5 & 96 & 1856 & 5 & 40 & $0.2262 E-04$ & $0.1549 E-04$ & 0.685 & $0.1261 E-04$ & 0.557 & $11-2$ \\
\hline 694 & 2 & 5 & 106 & 1866 & 3 & 49 & $0.1896 E-04$ & $0.1098 E-04$ & 0.579 & $0.9542 E-05$ & 0.503 & L $1-2$ \\
\hline 695 & 2 & 5 & 116 & 1876 & 4 & 47 & $0.1756 E-04$ & $0.1239 E-04$ & 0.706 & $0.1070 E-04$ & 0.609 & L $1-2$ \\
\hline 696 & 2 & 5 & 126 & 1886 & 13 & 36 & $0.2871 \mathrm{E}-04$ & $0.2372 E-04$ & 0.826 & $0.1633 E-04$ & 0.569 & $\llcorner 1-2$ \\
\hline 697 & 2 & 5 & 136 & 1896 & 357 & 50 & $0.2859 E-04$ & $0.2381 E-04$ & 0.833 & $0.1962 E-04$ & 0.686 & L $1-2$ \\
\hline 698 & 2 & 5 & 146 & 1906 & 5 & 50 & $0.1472 E-04$ & $0.1212 E-04$ & 0.823 & $0.6915 E-05$ & 0.470 & L $1-2$ \\
\hline 699 & 2 & 6 & 6 & 1916 & 3 & 51 & $0.2533 E-04$ & $0.1826 E-04$ & 0.721 & & & L $1-2$ \\
\hline 700 & 2 & 6 & 16 & 1926 & 6 & 55 & $0.2626 E-04$ & $0.2161 E-04$ & 0.823 & $0.1568 E-04$ & 0.597 & L $1-2$ \\
\hline 701 & 2 & 6 & 26 & 1936 & 3 & 41 & $0.2142 E-04$ & $668 E-04$ & 0.779 & $0.1250 E-04$ & 0.584 & L $1-2$ \\
\hline 702 & 2 & 6 & 36 & 1946 & 8 & 41 & $0.2510 E-04$ & $0.1921 \mathrm{E}-04$ & 0.765 & $0.1393 E-04$ & 0.555 & ᄂ $1-2$ \\
\hline 703 & 2 & 6 & 46 & 1956 & 5 & 42 & $0.2327 E-04$ & $0.1834 E-04$ & 0.788 & $0.1253 E-04$ & 0.538 & L $1-2$ \\
\hline 704 & 2 & 6 & 56 & 1966 & 4 & 47 & $0.2256 E-04$ & $0.1510 E-04$ & 0.669 & $0.1198 E-04$ & 0.531 & $\lfloor 1-2$ \\
\hline 705 & 2 & 6 & 66 & 1976 & 360 & 51 & $0.3137 E-04$ & $0.2370 E-04$ & 0.756 & $0.1902 E-04$ & 0.606 & L $1-2$ \\
\hline 706 & 2 & 6 & 76 & 1986 & 4 & 46 & $0.1875 E-04$ & $0.1141 \mathrm{E}-04$ & 0.608 & $0.8260 E-05$ & 0.441 & L $1-2$ \\
\hline 707 & 2 & 6 & 86 & 1996 & 168 & -47 & $0.3568 E-05$ & $0.4439 E-05$ & 1.244 & $0.4510 E-05$ & 1.264 & L $1-2$ \\
\hline 708 & 2 & 6 & 96 & 2006 & 179 & -47 & $0.7811 \mathrm{E}-05$ & $0.1175 E-04$ & 1.505 & $0.9735 E-05$ & 1.246 & L $1-2$ \\
\hline 709 & 2 & 6 & 106 & 2016 & 7 & 49 & $0.1830 \mathrm{E}-04$ & $0.1102 E-04$ & 0.602 & $0.6503 E-05$ & 0.355 & L $1-2$ \\
\hline 710 & 2 & 6 & 116 & 2026 & 0 & 52 & $0.2458 E-04$ & $1520 E-$ & 619 & $0.9541 \mathrm{E}$ & 388 & $\llcorner 1-2$ \\
\hline 711 & 2 & 6 & 126 & 2036 & 119 & -19 & $0.7439 E-05$ & $8339 E-06$ & 0.112 & $0.5363 E-06$ & 0.072 & L3 \\
\hline 712 & 2 & 6 & 136 & 2046 & 170 & -53 & $0.6789 E-05$ & $0.1039 E-04$ & 1.531 & $0.9320 E-05$ & 1.373 & $\llcorner 1-2$ \\
\hline 713 & 2 & 6 & 146 & 2056 & 56 & -62 & $0.5867 E-05$ & $0.6582 E-06$ & 0.112 & $0.4942 E-06$ & 0.084 & L $2-3$ \\
\hline 714 & 2 & 7 & 6 & 2066 & 341 & 66 & $0.1066 E-04$ & $0.7012 E-05$ & 0.658 & $0.4429 E-05$ & 0.416 & L $1-5$ \\
\hline 715 & 2 & 7 & 16 & 2076 & 346 & 48 & $0.7562 E-05$ & $0.5553 E-05$ & 0.734 & $0.4033 E-05$ & 0.533 & L $1-2$ \\
\hline 716 & 2 & 7 & 26 & 2086 & 288 & 77 & $0.14 .25 E-04$ & $9334 E-05$ & 0.655 & $0.6670 E-05$ & 0.468 & L $1-7$ \\
\hline 717 & 2 & 7 & 36 & 2096 & 347 & 66 & $0.2024 E-04$ & $0.13775-04$ & 0.681 & $0.9337 E-05$ & 0.461 & L $1-2$ \\
\hline 718 & 3 & 1 & 6 & 2116 & 298 & 33 & $0.9963 E-05$ & $0.4398 E-05$ & 0.441 & $0.2794 E-05$ & 0.280 & L $1-2$ \\
\hline 719 & 3 & 1 & 16 & 2126 & 309 & 42 & $0.1476 E-04$ & $0.6263 E-05$ & 0.424 & $0.4549 \varepsilon-05$ & 0.308 & $-1-2$ \\
\hline 720 & 3 & 1 & 26 & 2136 & 120 & -65 & $0.3283 E-05$ & $0.4179 E-05$ & 1.273 & $0.2981 E-05$ & 0.908 & L $1-2$ \\
\hline 721 & 3 & 1 & 36 & 2146 & 128 & -54 & $0.8681 E-06$ & $0.5440 E-05$ & 6.266 & $0.3948 \Xi-05$ & 4.548 & ᄂ $1-2$ \\
\hline 722 & 3 & 1 & 86 & 2196 & 329 & 45 & $0.9741 E-05$ & $0.6017 E-05$ & 0.618 & $0.3296 E-05$ & 0.338 & $\llcorner 1-2$ \\
\hline 723 & 3 & 1 & 46 & 2156 & 146 & -50 & $0.2864 E-05$ & $0.6617 E-05$ & 2.310 & $0.5712 E-05$ & 1.994 & L $1-2$ \\
\hline 724 & 3 & 1 & 56 & 2166 & 142 & -56 & $0.2264 E-05$ & $0.6562 E-05$ & 2.898 & $0.5742 E-05$ & 2.536 & L $1-2$ \\
\hline
\end{tabular}


Table 2. (Continued).

\begin{tabular}{|c|c|c|c|c|c|c|c|c|c|c|c|c|}
\hline 兽 & ذِّ & 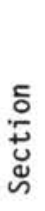 & 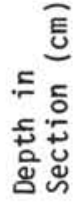 & 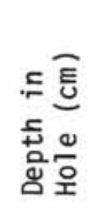 & 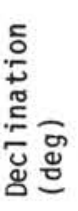 & 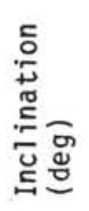 & NRM (emu) & ${ }^{\mathrm{J}} 100$ (emu) & ${ }^{3}{ }_{100} / J_{0}$ & ${ }^{J}{ }_{200}(\mathrm{emu})$ & ${ }^{\mathrm{J}} 200^{/ \mathrm{J}_{0}}$ & Note \\
\hline 725 & 3 & 1 & 66 & 2176 & 140 & -53 & $0.2922 E-05$ & $0.6975 E-05$ & 2.387 & $0.5628 \mathrm{E}-05$ & 1.926 & ᄂ $1-2$ \\
\hline 726 & 3 & 1 & 76 & 1996 & 169 & -73 & $0.5738 E-05$ & $0.3001 E-06$ & 0.052 & $0.36895-06$ & 0.064 & \\
\hline 727 & 3 & 1 & 96 & 2016 & 342 & 42 & $0.9242 E-05$ & $0.4551 E-05$ & 0.492 & $0.2827 E-05$ & 306 & \\
\hline 728 & 3 & 1 & 106 & 2026 & 134 & -53 & $55 E-05$ & $64 E-05$ & 665 & $77 E-05$ & 517 & $\llcorner 1-2$ \\
\hline 729 & 3 & 1 & 116 & 2036 & 152 & -54 & $0.1359 E-05$ & $0.5320 E-05$ & 3.915 & $0.3883 E-05$ & 2.858 & L $1-2$ \\
\hline 730 & 3 & 1 & 126 & 2046 & 149 & -42 & $0.2032 E-05$ & $0.7549 E-05$ & 3.715 & $0.58705-05$ & 2.889 & $41-2$ \\
\hline 731 & 3 & 1 & 136 & 2056 & 154 & -54 & $0.2386 E-05$ & $0.6185 E-05$ & 2.592 & $0.4220 E-05$ & 1.769 & $\llcorner 1-2$ \\
\hline 732 & 3 & 1 & 146 & 2066 & 332 & 37 & $0.1283 E-04$ & $0.5884 E-05$ & 0.459 & $0.4390 E-05$ & 0.342 & $\llcorner 1-2$ \\
\hline 733 & 3 & 2 & 6 & 2076 & 351 & 41 & $0.1747 E-04$ & $0.9174 E-05$ & 0.525 & $0.5492 E-05$ & 0.314 & $\llcorner 1-2$ \\
\hline 734 & 3 & 2 & 16 & 2086 & 337 & 45 & & $0.6428 E-05$ & & $0.4346 E-05$ & & $1-2$ \\
\hline 735 & 3 & 2 & 26 & 2096 & 347 & 53 & $0.1141 E-04$ & $0.6032 E-05$ & 0.529 & $0.3613 E-05$ & 0.317 & L $1-2$ \\
\hline 736 & 3 & 2 & 36 & 2106 & 345 & 39 & $0.1153 E-04$ & $0.6126 E-05$ & 0.531 & $0.4434 E-05$ & 0.385 & \\
\hline 737 & 3 & 2 & 46 & 2116 & 352 & 47 & $0.1324 E-04$ & $0.7074 E-05$ & 0.534 & $0.5032 E-05$ & 0.380 & \\
\hline 738 & 3 & 2 & 56 & 2126 & 351 & 52 & $0.1262 E-04$ & $7451 E-05$ & 0.590 & $27 E-05$ & 0.303 & \\
\hline 739 & 3 & 2 & 66 & 2136 & 161 & -49 & $0.4161 E-05$ & $8 E-05$ & 0.394 & $8 E-05$ & 0.346 & -2 \\
\hline 740 & 3 & 2 & 76 & 2206 & 153 & -49 & $9 E-05$ & $8 E-05$ & 0.603 & E- & 0.553 & $\perp 1-2$ \\
\hline 741 & 3 & 2 & 86 & 2216 & 146 & -52 & $0.2801 E-05$ & $5 E-05$ & .787 & $E-$ & 1.281 & $\perp 1-2$ \\
\hline 742 & 3 & 2 & 96 & 2226 & 153 & -49 & $9 E-05$ & $76 E-05$ & 2.627 & $0.2650 E-05$ & 2.192 & ᄂ $1-2$ \\
\hline 743 & 3 & 2 & 106 & 2236 & 162 & -43 & $0.1169 E-$ & $7 E-05$ & 3.241 & $0.3408 E-05$ & 2.916 & $L 1-2$ \\
\hline 744 & 3 & 2 & 116 & 2246 & 169 & -56 & $E-05$ & $0.5882 E-05$ & 5.403 & $0.4196 E-05$ & 3.854 & $\leq 1-2$ \\
\hline 745 & 3 & 2 & 126 & 2256 & 161 & -36 & $0.1544 \subseteq-05$ & $E-05$ & 2.854 & $0.3693 E-05$ & 2.392 & L $1-2$ \\
\hline 746 & 3 & 2 & 136 & 2266 & 161 & -37 & $0.2920 E-05$ & $1 E-05$ & 2.230 & $0.4978 E-05$ & 1.705 & $\llcorner 1-2$ \\
\hline 747 & 3 & 2 & 146 & 2276 & 162 & -38 & $0.2444 E-05$ & $6 E-05$ & 2.855 & $0.5204 E-05$ & 2.129 & $\llcorner 1-2$ \\
\hline 748 & 3 & 3 & 6 & 2286 & 186 & 41 & $0.5170 E-05$ & $0.9350 E-05$ & 1.808 & $0.5610 E-05$ & 1.085 & L $1-2$ \\
\hline 749 & 3 & 3 & 16 & 2296 & 182 & -47 & $0.2157 E-05$ & $0.2741 E-05$ & 1.271 & $0.2042 E-05$ & 0.947 & \\
\hline 750 & 3 & 3 & 26 & 2306 & 161 & -37 & $0.2856 E-05$ & $0.1763 E-05$ & 0.617 & $0.1365 E-05$ & 0.478 & \\
\hline 751 & 3 & 3 & 36 & 2316 & 158 & -33 & $0.4506 \mathrm{E}-05$ & $0.7157 \mathrm{E}-05$ & 1.588 & $0.5529 E-05$ & 1.227 & \\
\hline 752 & 3 & 3 & 46 & 2326 & 106 & 16 & $0.9670 E-05$ & $0.6476 E-05$ & 0.670 & 0.4 & 6 & \\
\hline 765 & 3 & 6 & 26 & 2452 & 355 & 54 & $E-04$ & $0.1671 E-05$ & 0.133 & 0 . & 053 & \\
\hline 766 & 3 & 6 & 46 & 2462 & 131 & -44 & 0. & 550 & 1.402 & 5 & 1.179 & -2 \\
\hline 767 & 3 & 6 & 66 & 2492 & 63 & 74 & $0.1237 \mathrm{E}-04$ & $3 E-06$ & 0.081 & $0.3768 E-06$ & 0.030 & L 1 \\
\hline 768 & 3 & 6 & 86 & 2512 & 316 & 55 & $0.1670 E-04$ & $0.5049 E-05$ & 0.302 & $0.3067 E-05$ & 0.184 & L $1-2$ \\
\hline 769 & 3 & 6 & 106 & 2532 & 134 & -46 & $0.7143 E-05$ & $0.5366 E-05$ & 0.751 & $0.4295 E-05$ & 0.601 & $L 1-2$ \\
\hline 770 & 3 & 6 & 126 & 2552 & 139 & 4 & $0.1578 E-04$ & $0.1296 E-05$ & 0.082 & $0.9644 E-06$ & 0.061 & $L$ \\
\hline 771 & 3 & 6 & 146 & 2572 & 117 & -25 & $0.7950 E-05$ & $0.2499 E-05$ & 0.314 & $0.2611 E-05$ & 0.328 & $41-2$ \\
\hline 772 & 3 & 7 & 6 & 2582 & 120 & 66 & $0.1303 E-04$ & $0.1070 E-05$ & 0.082 & $0.3924 E-06$ & 0.030 & 1 \\
\hline 773 & 3 & 7 & 26 & 2602 & 345 & 63 & $0.1582 E-04$ & $0.1490 E-05$ & 0.094 & $0.5079 E-06$ & 0.032 & \\
\hline 774 & 3 & 7 & 46 & 2622 & 324 & 52 & $0.1407 E-04$ & $0.3479 E-05$ & 0.2 .47 & $0.1844 E-05$ & 0.131 & \\
\hline 775 & 4 & 3 & 106 & 3416 & 12 & 18 & $0.14805-04$ & $0.6264 E-05$ & 0.423 & 0. & & \\
\hline 776 & 4 & 3 & 126 & 3436 & 7 & -11 & $0.8827 E-05$ & $0.5145 E-05$ & 0.583 & 0. & 7 & 3 \\
\hline 777 & 4 & 3 & 146 & 3456 & 7 & 19 & $44 E-04$ & $0.3097 E-05$ & 0.230 & 0. & 18 & 3 \\
\hline 778 & 4 & 4 & 6 & 3466 & 2 & 16 & $E-04$ & $E-05$ & 0.238 & 5 & 0.121 & $\lfloor 1-3$ \\
\hline 779 & 4 & 4 & 26 & 3486 & 3 & 12 & & 5 & .247 & $4 E-05$ & 0.126 & $-1-3$ \\
\hline 780 & 4 & 4 & 46 & 3506 & 353 & 23 & $0.1517 E-04$ & $0.3571 E-05$ & 0.235 & $0.1805 E-05$ & 0.119 & $\llcorner 1-3$ \\
\hline 781 & 4 & 4 & 66 & 3526 & 5 & 22 & $0.1199 \mathrm{E}-04$ & $0.2473 E-05$ & 0.206 & $0.1354 \subseteq-05$ & 0.113 & L $1-3$ \\
\hline 782 & 4 & 4 & 86 & 3546 & 337 & 32 & $0.1223 E-04$ & $0.3474 E-05$ & 0.284 & $0.1906 E-05$ & 0.156 & $\llcorner 1-3$ \\
\hline 783 & 4 & 4 & 106 & 3566 & 342 & 29 & $0.1447 E-04$ & $0.3357 E-05$ & 0.232 & $0.1996 \mathrm{E}-05$ & 0.138 & $L 1-3$ \\
\hline 784 & 4 & 4 & 126 & 3586 & 342 & 37 & $0.1759 E-04$ & $0.4362 E-05$ & 0.248 & $0.2146 \mathrm{E}-05$ & 22 & $L$ \\
\hline 785 & 4 & 4 & 146 & 3606 & 11 & 17 & $0.1496 E-04$ & $3091 \leq-05$ & 207 & 5 & & \\
\hline 786 & 4 & 5 & 6 & 3616 & 327 & -15 & $0.1456 E-04$ & $0.5362 E-05$ & 0.368 & 5 & 0.208 & \\
\hline 787 & 4 & 5 & 26 & 3636 & 355 & 17 & $0.1528 E-04$ & $.3090 E-05$ & 0.202 & $0.1789 \mathrm{E}-05$ & 0.117 & \\
\hline 788 & 4 & 5 & 46 & 3656 & 349 & 24 & $0.2030 E-04$ & $29245-05$ & 0.144 & $0.1784 E-05$ & 0.088 & $-1-3$ \\
\hline 789 & 4 & 5 & 66 & 3676 & 18 & -18 & $0.1606 \mathrm{E}-04$ & $0.2439 E-05$ & 0.152 & $0.1842 E-05$ & 0.115 & $1-1-3$ \\
\hline 790 & 4 & 5 & 86 & 3696 & 27 & -25 & $0.16535-04$ & $0.40225-05$ & 0.243 & $0.3460 E-05$ & 0.209 & $\llcorner 1-3$ \\
\hline 791 & 4 & 5 & 106 & 3716 & 5 & -18 & $0.1594 \mathrm{E}-04$ & $0.6014 \subseteq-05$ & 0.377 & $0.4083 E-05$ & 0.256 & L $1-3$ \\
\hline 792 & 4 & 5 & 126 & 3736 & & & & $9 E-05$ & & & & $U$ \\
\hline 793 & 4 & 5 & 146 & 3756 & 354 & 15 & $0.1007 E-04$ & $0.5496 E-05$ & 0.546 & $0.3969 E-05$ & 0.394 & L $1-3$ \\
\hline 794 & 4 & 6 & 6 & 3766 & 360 & -11 & $0.1045 E-04$ & $0.5869 E-05$ & 0.562 & $0.3308 \mathrm{E}-05$ & 0.317 & L $1-3$ \\
\hline 795 & 4 & 6 & 26 & 3786 & 6 & -14 & $0.8672 E-05$ & $0.5609 E-05$ & 0.647 & $0.3583 E-05$ & 0.413 & L $1-3$ \\
\hline 796 & 4 & 6 & 46 & 3806 & 352 & -10 & $0.8328 E-05$ & $0.7404 E-05$ & 0.889 & $0.46295-05$ & 0.556 & ᄂ $1-4$ \\
\hline
\end{tabular}


Table 2. (Continued).

\begin{tabular}{|c|c|c|c|c|c|c|c|c|c|c|c|c|}
\hline 咅 & ذั) & 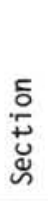 & 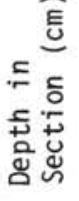 & 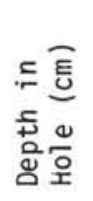 & 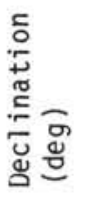 & 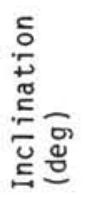 & NRM (emu) & ${ }^{3} 100$ (emu) & ${ }^{\mathrm{J}} 100^{/{ }^{3}} 0$ & ${ }^{J} 200(\mathrm{emu})$ & ${ }^{{ }} 200^{/ J_{0}}$ & Note \\
\hline 797 & 4 & 6 & 66 & 3826 & 2 & -49 & $0.8676 E-05$ & $0.8458 E-05$ & 0.975 & $0.5881 E-05$ & 0.678 & L $1-2$ \\
\hline 798 & 4 & 6 & 86 & 3846 & 10 & -33 & $0.1688 E-05$ & $0.5970 E-05$ & 3.536 & $0.4756 E-05$ & 2.817 & \\
\hline 799 & 4 & 6 & 106 & 3866 & 31 & -15 & $0.6964 E-05$ & $0.6753 E-05$ & 0.970 & $0.4012 E-05$ & 0.576 & \\
\hline 800 & 4 & 6 & 126 & 3886 & 26 & -5 & $0.1244 E-04$ & $0.1066 E-04$ & 0.857 & $0.69705-05$ & 0.560 & \\
\hline 801 & 4 & 6 & 146 & 3906 & 270 & 12 & $0.3239 E-04$ & $0.1363 E-04$ & 0.421 & $0.9374 E-05$ & 0.289 & L $1-7$ \\
\hline 802 & 4 & 7 & 6 & 3915 & 269 & 56 & $0.3634 E-04$ & $0.1803 E-04$ & 0.496 & $0.1285 E-04$ & 0.353 & $1.1-2$ \\
\hline 803 & 4 & 7 & 26 & 3936 & 336 & 37 & $0.2354 \mathrm{E}-04$ & $384 E-05$ & 0.229 & $0.3126 E-05$ & 0.133 & L $1-2$ \\
\hline 804 & 4 & 7 & 46 & 3957 & 54 & 42 & $0.1938 \mathrm{E}-04$ & $0.6843 E-05$ & 0.353 & $0.5074 E-05$ & 0.262 & ! $1-2$ \\
\hline 806 & 5 & 2 & 6 & 4016 & 103 & 29 & $0.1632 E-04$ & $0.8228 \mathrm{E}-05$ & 0.504 & $0.6278 E-05$ & 0.385 & $\llcorner 1-3$ \\
\hline 807 & 5 & 2 & 26 & 4036 & 105 & 46 & $0.3435 E-04$ & $0.1370 E-04$ & 0.399 & $0.9721 \mathrm{E}-05$ & 0.283 & L $1-2$ \\
\hline 808 & 5 & 2 & 46 & 4056 & 97 & 49 & $0.3040 E-04$ & $0.1011 E-04$ & 0.333 & $0.5747 E-05$ & 0.189 & L $1-2$ \\
\hline 809 & 5 & 2 & 67 & 4077 & 90 & 16 & $0.23315-04$ & $0.8137 E-05$ & 0.349 & $0.47945-05$ & 0.206 & ᄂ $1-2$ \\
\hline 810 & 5 & 2 & 87 & 4097 & 110 & 18 & $0.2265 E-04$ & $0.76025-05$ & 0.336 & $0.5233 E-05$ & 0.231 & L $1-3$ \\
\hline 811 & 5 & 2 & 107 & 4117 & 105 & 32 & $0.1540 E-04$ & $0.6051 \mathrm{E}-05$ & 0.393 & 0.35 & 0.228 & L $1-2$ \\
\hline 812 & 5 & 2 & 126 & 4136 & 121 & 28 & $24 E-04$ & $0.3696 E-05$ & 0.214 & $E-05$ & 0.145 & $-1-2$ \\
\hline 813 & 5 & 2 & 146 & 4156 & 98 & 60 & 0.26 & $0.6526 E-05$ & 0.247 & $0.4259 E-05$ & 0.161 & $\llcorner 1-2$ \\
\hline 814 & 5 & 3 & 6 & 4166 & 103 & 58 & $0.2998 E-04$ & $0.9982 E-05$ & 0.333 & $0.6253 E-05$ & 0.209 & $\perp-1-2$ \\
\hline 815 & 5 & 3 & 26 & 4186 & 3 & 64 & $0.1633 E-04$ & $0.5688 E-05$ & 0.348 & $0.3578 E-05$ & 0.219 & $1-1-2$ \\
\hline 816 & 5 & 3 & 46 & 4206 & 79 & -23 & $0.1483 E-04$ & $0.5437 E-05$ & 0.367 & $0.2913 E-05$ & 0.196 & L 1 \\
\hline 817 & 5 & 3 & 66 & 4226 & 261 & 2 & $0.1789 E-04$ & $0.9223 E-05$ & 0.516 & $0.2755 E-05$ & 0.154 & $\llcorner 1-3$ \\
\hline 818 & 5 & 3 & 86 & 4246 & 89 & -13 & $0.1618 E-04$ & $0.5585 E-05$ & 0.345 & $0.3848 E-05$ & 0.238 & $41-3$ \\
\hline 819 & 5 & 3 & 106 & 4266 & 97 & 20 & $0.4027 E-04$ & $0.1294 E-04$ & 0.321 & $0.5921 \mathrm{E}-05$ & 0.147 & L \\
\hline 820 & 5 & 3 & 126 & 4286 & 289 & 44 & $0.1613 E-04$ & $0.5407 E-05$ & 0.335 & $0.5262 E-05$ & 0.326 & 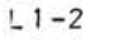 \\
\hline 821 & 5 & 3 & 146 & 4306 & 74 & 24 & $0.1564 E-04$ & $0.12705-04$ & 0.812 & 0.98 & 0.629 & $\llcorner 1-2$ \\
\hline 823 & 5 & 4 & 26 & 4336 & 107 & 32 & $E-04$ & $0.1154 E-04$ & 0.278 & $0.5199 E-05$ & 0.125 & $\llcorner 1-2$ \\
\hline 824 & 5 & 4 & 46 & 4356 & 85 & 36 & $0.2586 E-04$ & $0.9017 E-05$ & 0.349 & $0.4917 E-05$ & 0.190 & L $1-2$ \\
\hline 825 & 5 & 4 & 66 & 4376 & & & $0.1242 E-04$ & $1.1595 E-05$ & 0.128 & $2.8791 E-06$ & 0.071 & $u$ \\
\hline 826 & 5 & 4 & 86 & 4396 & 273 & -13 & 0.266 & $0.25765-04$ & 0.965 & $0.1256 E-04$ & 0.471 & $\bigsqcup 1-2$ \\
\hline 827 & 5 & 4 & 106 & 4416 & 96 & 13 & 0. & $0.4960 E-05$ & 0.187 & $0.7229 E-06$ & 0.027 & L 1 \\
\hline 828 & 5 & 4 & 126 & 4436 & 96 & 41 & 0 . & $0.8733 E-05$ & 0.286 & $0.2449 \subseteq-05$ & 0.080 & $\lfloor 1-2$ \\
\hline 829 & 5 & 4 & 146 & 4456 & 315 & 44 & $0.2864 E-04$ & $0.5956 E-05$ & 0.208 & $0.4706 E-05$ & 0.164 & $\llcorner 1-2$ \\
\hline 830 & 5 & 5 & 6 & 4466 & 61 & 43 & $0.2244 E-04$ & $0.2971 \subseteq-05$ & 0.132 & $0.8105 E-06$ & 0.036 & -1 \\
\hline 831 & 5 & 5 & 26 & 4486 & 94 & 53 & $0.2914 E-04$ & $0.1132 E-04$ & 0.389 & $0.4192 \mathrm{E}-05$ & 0.144 & \\
\hline 832 & 5 & 5 & 46 & 4506 & 301 & 2 & $0.7380 E-05$ & $0.3889 E-05$ & 0.527 & $0.3905 E-05$ & 0.529 & ᄂ $1-2$ \\
\hline 833 & 5 & 5 & 66 & 4526 & 8 & -46 & $0.1286 E-04$ & $0.1913 E-05$ & 0.149 & $7 E-05$ & 0.162 & $\llcorner 1-2$ \\
\hline 834 & 5 & 5 & 86 & 4546 & 273 & -6 & $0.1232 E-04$ & $0.1129 E-04$ & 0.917 & 0.67 & 0.552 & $\checkmark 1$ \\
\hline 835 & 5 & 5 & 107 & 4567 & 109 & 13 & $0.4942 E-04$ & $0.2692 E-04$ & 0.545 & $E=04$ & 0.331 & 11 \\
\hline 836 & 5 & 5 & 126 & 4586 & 257 & -9 & 0.1 & 0.34076 & 0.269 & $7 E-05$ & 0.172 & L $1-4$ \\
\hline 837 & 5 & 5 & 146 & 4606 & 281 & -34 & 0. & $0.1212 E-04$ & 1.256 & $0.5552 E-05$ & 0.575 & L $1-2$ \\
\hline 838 & 5 & 6 & 6 & 4616 & 262 & -17 & $0.1514 \mathrm{E}-04$ & $0.1603 E-04$ & 1.059 & $0.1013 E-04$ & 0.669 & L. $1-3$ \\
\hline 839 & 5 & 6 & 26 & 4636 & 259 & 1 & $0.1811 E-04$ & $0.1821 E-04$ & 1.006 & $0.1091 \mathrm{E}-04$ & 0.603 & $41-3$ \\
\hline 840 & 5 & 6 & 46 & 4656 & 283 & 0 & $0.1976 E-04$ & $0.2064 E-04$ & 1.045 & $0.1061 E-04$ & 0.537 & $-1-2$ \\
\hline 841 & 5 & 6 & 66 & 4676 & 280 & -6 & $0.2812 E-04$ & $0.2301 E-04$ & 0.818 & $0.15175-04$ & 0.540 & ᄂ $1-2$ \\
\hline 842 & 5 & 6 & 86 & 4696 & 243 & -3 & $0.1420 E-04$ & $0.9403 E-05$ & 0.662 & $0.4469 E-05$ & 0.315 & ᄂ $1-2$ \\
\hline 843 & 5 & 6 & 106 & 4716 & 57 & 19 & $0.2690 E-04$ & $0.6006 E-05$ & 0.223 & $0.4380 E-05$ & 0.163 & L 1 \\
\hline 844 & 6 & 1 & 126 & 5122 & 26 & -57 & $0.2155 E-04$ & $0.8238 E-05$ & 0.382 & $0.6188 E-05$ & 0.287 & L 1 \\
\hline 845 & 6 & 1 & 146 & 5142 & 263 & -22 & $0.3091 E-04$ & $0.6020 E-05$ & 0.195 & $0.3932 E-05$ & 0.127 & $41-2$ \\
\hline 846 & 6 & 2 & 6 & 5162 & 312 & -8 & $0.2003 E-04$ & $521 E-05$ & 76 & $E-05$ & 0.171 & ᄂ $1-2$ \\
\hline 847 & 6 & 2 & 26 & 5182 & 47 & -12 & $0.3040 \mathrm{E}-0.4$ & $0.7982 E-05$ & 0.263 & $0.4810 E-05$ & 0.158 & ᄂ $1-2$ \\
\hline 848 & 6 & 2 & 46 & 5202 & 232 & 30 & $0.5203 E-04$ & $0.1352 E-04$ & 0.260 & $0.83745-05$ & 0.161 & L $1-2$ \\
\hline 849 & 6 & 2 & 66 & 5222 & 237 & 17 & $0.5246 E-04$ & $0.1408 E-04$ & 0.268 & $0.1014 \mathrm{E}-04$ & 0.193 & ᄂ 1-2 \\
\hline 850 & 6 & 2 & 86 & 5242 & 278 & -13 & $0.3722 E-04$ & $0.4717 E-05$ & 0.127 & $0.2590 E-05$ & 0.070 & L $1-2$ \\
\hline 851 & 6 & 2 & 106 & 5262 & 244 & 46 & $0.3808 E-04$ & $0.5654 E-05$ & 0.148 & $0.3243 E-05$ & 0.085 & $41-2$ \\
\hline 852 & 6 & 2 & 126 & 5282 & 261 & 20 & $0.3825 E-04$ & $0.3968 \varepsilon-05$ & 0.104 & $0.2852 E-05$ & 0.075 & L $1-4$ \\
\hline 853 & 6 & 2 & 146 & 5302 & 52 & 7 & $0.32575-04$ & $0.3611 E-05$ & 0.111 & $0.2009 E-05$ & 0.062 & $41-2$ \\
\hline 854 & 6 & 3 & 6 & 5312 & 27 & -19 & $0.2570 E-04$ & $0.7921 E-05$ & 0.308 & $0.5690 E-05$ & 0.221 & $-1-2$ \\
\hline 855 & 6 & 3 & 26 & 5322 & 257 & 40 & $0.3434 E-04$ & $0.4186 E-05$ & 0.122 & $0.3185 E-05$ & 0.093 & $\lfloor 1-2$ \\
\hline 856 & 6 & 3 & 46 & 5342 & 131 & 58 & $0.3327 \varepsilon-04$ & $0.3897 E-05$ & 0.117 & $0.2475 E-05$ & 0.074 & $-1-2$ \\
\hline 857 & 6 & 3 & 67 & 5363 & 260 & 20 & $0.4743 E-04$ & $0.1023 \subseteq-04$ & 0.216 & $0.6504 E-05$ & 0.137 & ᄂ $1-2$ \\
\hline
\end{tabular}




\begin{tabular}{|c|c|c|c|c|c|c|c|c|c|c|c|c|}
\hline 号 & ปั้ & 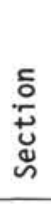 & 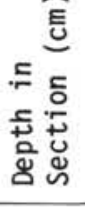 & $\begin{array}{l}\text { 틍 } \\
\text { 동 } \\
\text { 웜우 }\end{array}$ & 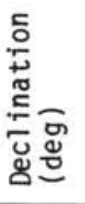 & 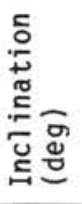 & NRM (emu) & ${ }^{3}{ }_{100}(\mathrm{emu})$ & ${ }^{3}{ }_{100} /{ }^{3} 0$ & ${ }^{J_{200}}(\mathrm{emu})$ & ${ }^{\mathrm{J}} 200^{/ \mathrm{J}_{0}}$ & Note \\
\hline 858 & 6 & 3 & 86 & 5382 & 32 & 2 & $0.3146 E-04$ & $0.6622 E-05$ & 0.211 & $0.3959 E-05$ & 0.126 & $1-1-2$ \\
\hline 859 & 6 & 3 & 106 & 5402 & 48 & -13 & $0.2716 \mathrm{E}-04$ & $0.7798 E-05$ & 0.287 & $0.5935 \bar{\varepsilon}-05$ & 0.219 & L $1-2$ \\
\hline 860 & 6 & 3 & 126 & 5422 & 70 & -25 & $0.2424 E-04$ & $0.1590 E-04$ & 0.656 & $0.1150 E-04$ & 0.474 & $\lfloor 1-2$ \\
\hline 861 & 6 & 3 & 146 & 5442 & 262 & -55 & $0.3211 E-04$ & $0.5615 E-05$ & 0.175 & $0.2364 E-05$ & 0.074 & $1-1-2$ \\
\hline 862 & 6 & 4 & 6 & 5452 & 324 & 13 & $0.2343 E-04$ & $0.4006 E-05$ & 0.171 & $0.2551 \mathrm{E}-05$ & 0.109 & -1 \\
\hline 863 & 6 & 4 & 26 & 5472 & 215 & 38 & $0.3452 E-04$ & $0.3926 E-05$ & 0.114 & $0.1129 E-05$ & 0.033 & $\llcorner 1$ \\
\hline 864 & 6 & 4 & 46 & 5492 & 258 & 12 & $0.5312 E-04$ & $0.1479 E-04$ & 0.278 & $0.10705-04$ & 0.201 & -2 \\
\hline 865 & 6 & 4 & 66 & 5512 & 87 & -22 & $0.3780 E-04$ & $0.8017 E-06$ & 0.021 & $0.2888 E-05$ & 0.076 & \\
\hline 866 & 6 & 4 & 86 & 5532 & 253 & -5 & $0.4840 \bar{E}-04$ & $0.4384 E-05$ & 0.091 & $0.15425-05$ & 0.032 & ᄂ 1 \\
\hline 867 & 6 & 4 & 106 & 5552 & 304 & 27 & $0.4988 \varepsilon-04$ & $0.1199 E-04$ & 0.240 & $2 E-05$ & 0.173 & ᄂ $1-2$ \\
\hline 868 & 6 & 4 & 146 & 5592 & 275 & 17 & $0.7992 E-04$ & $0.4446 E-04$ & 0.556 & $0.3180 E-04$ & 0.398 & $-1-2$ \\
\hline 869 & 6 & 5 & 55 & 5651 & 273 & 22 & $0.5306 E-04$ & $0.1666 E-04$ & 0.314 & $0.1231 \mathrm{E}-04$ & 0.232 & $\leq 1-2$ \\
\hline 870 & 6 & 5 & 86 & 5682 & 275 & 19 & $0.7436 E-04$ & $0.35355-04$ & 0.475 & $0.2620 E-04$ & 0.352 & $\llcorner 1-2$ \\
\hline 871 & 6 & 5 & 106 & 5702 & 306 & 27 & $0.3562 E-04$ & $0.5017 E-05$ & 0.141 & $0.2708 E-05$ & 0.076 & ! $1-2$ \\
\hline 872 & 6 & 5 & 126 & 5722 & 83 & -11 & $0.2257 E-04$ & $0.1265 E-04$ & 0.561 & $0.9275 E-05$ & .411 & $\llcorner 1-2$ \\
\hline 873 & 6 & 5 & 144 & 5740 & 290 & 7 & $0.3917 E-04$ & $0.1761 \mathrm{E}-04$ & 0.450 & $0.1277 \mathrm{E}-04$ & 0.326 & $\lfloor 1-2$ \\
\hline 874 & 6 & 6 & 47 & 5793 & 278 & 4 & $0.40975-04$ & $0.1252 E-04$ & 0.306 & $0.6730 E-05$ & 0.164 & 1 \\
\hline 875 & 6 & 6 & 76 & 5822 & 57 & -6 & $0.35265-04$ & $0.1775 E-05$ & 0.050 & $0.3466 E-05$ & 0.098 & \\
\hline 876 & 6 & 6 & 136 & 5882 & 69 & -24 & $8 \subseteq-04$ & $3 E-05$ & 0.145 & 5 & 68 & $\llcorner 1-2$ \\
\hline 877 & 7 & 3 & 145 & 6245 & 162 & -21 & $E-04$ & $0.4494 E-04$ & 1.494 & 0.31 & 1.050 & $-1-2$ \\
\hline 878 & 7 & 4 & 91 & 6341 & 152 & -4 & $0.2487 E-04$ & $0.6307 E-05$ & 0.254 & $0.6090 E-05$ & 0.245 & $L 1-2$ \\
\hline 879 & 7 & 4 & 128 & 6378 & 339 & 12 & $0.3786 E-04$ & $0.5319 E-05$ & 0.140 & $0.2401 E-05$ & 0.063 & 41 \\
\hline 880 & 7 & 5 & 79 & 6479 & 119 & -14 & $0.2868 E-04$ & $0.2459 E-04$ & 0.858 & $0.1776 E-04$ & 0.619 & $\lfloor 1-2$ \\
\hline 881 & 7 & 6 & 26 & 6576 & 103 & -17 & $0.1395 E-04$ & $0.2372 E-04$ & 1.701 & $0.1821 E-04$ & 1.305 & $\llcorner 1-2$ \\
\hline 882 & 7 & 6 & 46 & 6596 & 115 & 70 & $0.1495 E-04$ & $0.1041 \Xi-04$ & 0.697 & $0.9306 E-05$ & 0.623 & $\lfloor 1-2$ \\
\hline 883 & 7 & 6 & 89 & 6639 & 275 & 5 & $0.3872 E-04$ & $0.1461 E-04$ & 0.377 & $0.8647 E-05$ & 0.223 & $-1-2$ \\
\hline 884 & 7 & 7 & 30 & 6730 & 260 & -5 & $0.1769 E-04$ & $0.1442 E-04$ & 0.815 & $0.1187 E-04$ & 0.671 & $-1-2$ \\
\hline 885 & 8 & 2 & 81 & 6976 & 293 & 3 & $0.4411 E-04$ & $0.2268 \mathrm{E}-04$ & 0.514 & $0.1260 E-04$ & 0.286 & -2 \\
\hline 886 & 8 & 3 & 29 & 7074 & 318 & -2 & $0.3063 E-04$ & $0.8459 E-05$ & 0.276 & $0.4129 E-05$ & 0.135 & -2 \\
\hline 887 & 8 & 3 & 65 & 7110 & 316 & 5 & $0.3969 \varepsilon-04$ & $0.1472 E-04$ & 0.371 & $0.7835 E-05$ & 0.197 & \\
\hline 888 & 8 & 3 & 96 & 7141 & 310 & 13 & $0.5152 E-04$ & $0.2096 E-04$ & 0.407 & $0.1303 E-04$ & 0.253 & $-1-2$ \\
\hline 889 & 8 & 4 & 21 & 7216 & 333 & 8 & $0.6136 E-04$ & $0.3187 E-04$ & 0.519 & $0.1644 E-04$ & 0.268 & $\llcorner 1-2$ \\
\hline 890 & 8 & 4 & 41 & 7236 & 326 & 13 & $0.4531 \mathrm{E}-04$ & $0.1506 E-04$ & 0.332 & $0.7976 E-05$ & 0.176 & L $1-2$ \\
\hline 891 & 8 & 4 & 99 & 7294 & 132 & -6 & $0.1723 E-04$ & $0.3324 E-05$ & 0.193 & $0.2161 \mathrm{E}-05$ & 0.125 & $\llcorner 1-2$ \\
\hline 892 & 8 & 5 & 58 & 7403 & 142 & -20 & $0.3787 E-04$ & $0.3818 E-04$ & 1.008 & $0.2331 E-04$ & 0.615 & L $1-2$ \\
\hline 893 & 8 & 5 & 142 & 7487 & 136 & -15 & $0.1176 E-04$ & $0.7574 \mathrm{E}-05$ & 0.644 & $0.5679 E-05$ & 0.483 & L $1-2$ \\
\hline 894 & 8 & 6 & 44 & 7539 & 330 & 13 & $0.1350 E-04$ & $0.2052 \mathrm{E}-04$ & 1.520 & $0.1207 E-04$ & 0.894 & ᄂ $1-2$ \\
\hline
\end{tabular}

Note: $\mathrm{L}=$ demagnetization level or levels (x 100; vector averages) used to define declination and inclination.

$U=$ magnetically unstable samples. $F=$ flow-in, based on shipboard visual descriptions.

still high enough to prevent the "swamping" of detrital remanence by chemical remanence, as appears to have occurred at Site 576.

The NRM $\left(J_{0}\right)$ and $J_{100}$ values correlate very well for the magnetically stable samples above about $145 \mathrm{~m}$ (Fig. 10). At greater depths, however, NRM values increase, whereas $J_{100}$ values approach zero. The $J_{100} / J_{0}$ ratio (Fig. 10) shows a tight clustering of values above about $100 \mathrm{~m}$, where sedimentation rates exceed $12 \mathrm{~m} / \mathrm{m} . \mathrm{y}$., increased scatter but a good continuation of the shallower trend to about $145 \mathrm{~m}$, then scattered, very low values to the base of the section.

\section{DISCUSSION}

The magnetic data at both Sites 576 and 578 yield detailed age-depth curves (Figs. 2, 8). Differentiation of these curves yields sedimentation rates as a function of time (Fig. 11).

In both cases, the past $2 \mathrm{~m}$.y. has been a period of increased sedimentation, a phenomenon that has been observed across the North Pacific and that has been attributed to eolian transport of fine-grained glacial debris from Asia and, to a lesser extent, from North America. This explanation is supported by the remarkable similarity of the rate increases at Sites 576 and 578 (about $5-6 \mathrm{~m} / \mathrm{m} . \mathrm{y}^{2}$ during the Quaternary, in each case). Such a uniform increase is unlikely to reflect either changes in bottom transport, given the separation of the two sites by Shatsky Rise, or changes in surface transport, given the upstream distance to source areas, particularly at Site 576. The uniformity of the increase also is in striking contrast to the very different accumulation rate his- 
Table 3. Magnetization of Site 576 samples after AF demagnetization at intensities above 200 Oe.

\begin{tabular}{|c|c|c|c|c|c|c|c|c|c|}
\hline$\frac{\frac{0}{a}}{\frac{\text { E⿱ }}{n}}$ & ؛े & 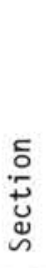 & 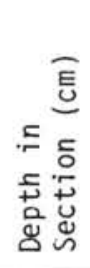 & 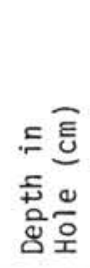 & 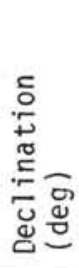 & 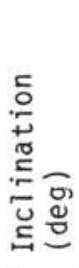 & 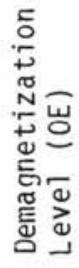 & J (emu) & $\mathrm{J} / \mathrm{J}_{0}$ \\
\hline 36 & 2 & 1 & 96 & 791 & 47 & -51 & $\begin{array}{l}300 \\
400\end{array}$ & $\begin{array}{l}0.1745 E-04 \\
0.1310 E-04\end{array}$ & $\begin{array}{l}0.689 \\
0.517\end{array}$ \\
\hline 45 & 2 & 2 & 36 & 881 & 263 & 19 & 300 & $0.1244 E-05$ & 0.199 \\
\hline 49 & 2 & 2 & 76 & 921 & 278 & 54 & $\begin{array}{l}300 \\
400\end{array}$ & $\begin{array}{l}0.1560 E-04 \\
0.1185 E-04\end{array}$ & $\begin{array}{l}0.582 \\
0.442\end{array}$ \\
\hline 53 & 2 & 2 & 116 & 961 & 267 & -38 & $\begin{array}{l}250 \\
300 \\
400\end{array}$ & $\begin{array}{l}0.6133 E-06 \\
0.3696 E-06 \\
0.6096 E-06\end{array}$ & $\begin{array}{l}0.214 \\
0.129 \\
0.212\end{array}$ \\
\hline 97 & 2 & 5 & 106 & 1401 & 287 & -69 & 300 & $0.5571 E-05$ & 0.756 \\
\hline 99 & 2 & 5 & 126 & 1421 & 154 & -67 & 300 & $0.1380 E-04$ & 0.644 \\
\hline 101 & 2 & 5 & 146 & 1441 & 185 & -84 & $\begin{array}{l}300 \\
400\end{array}$ & $\begin{array}{l}0.1349 E-04 \\
0.1066 E-04\end{array}$ & $\begin{array}{l}0.606 \\
0.479\end{array}$ \\
\hline 103 & 2 & 6 & 16 & 1461 & 75 & -79 & 300 & $0.1429 E-04$ & 0.656 \\
\hline 109 & 2 & 6 & 76 & 1521 & 22 & 14 & 300 & $0.1893 E-05$ & 0.499 \\
\hline 110 & 2 & 6 & 86 & 1531 & 20 & -20 & 300 & $0.7121 E-05$ & 0.501 \\
\hline 111 & 2 & 6 & 96 & 1541 & 6 & 28 & 300 & $0.6299 E-05$ & 0.435 \\
\hline 113 & 2 & 6 & 116 & 1561 & 329 & 19 & 300 & $0.5379 E-05$ & 0.575 \\
\hline 114 & 2 & 6 & 126 & 1571 & 335 & 19 & $\begin{array}{l}300 \\
400\end{array}$ & $\begin{array}{l}0.2906 E-05 \\
0.1669 E-05\end{array}$ & $\begin{array}{l}0.497 \\
0.286\end{array}$ \\
\hline 179 & 3 & 4 & 146 & 1581 & 162 & 40 & 300 & $0.3612 E-06$ & 0.065 \\
\hline 189 & 4 & 1 & 76 & 1856 & & & $\begin{array}{l}300 \\
400 \\
500 \\
600 \\
700 \\
800\end{array}$ & $\begin{array}{l}0.2121 E-05 \\
0.1489 E-04 \\
0.1091 E-05 \\
0.1138 E-05 \\
0.8307 E-06 \\
0.6931 E-06\end{array}$ & $\begin{array}{l}0.309 \\
2.171 \\
0.159 \\
0.166 \\
0.121 \\
0.101\end{array}$ \\
\hline 192 & 4 & 1 & 106 & 1886 & 146 & 51 & $\begin{array}{l}300 \\
400\end{array}$ & $\begin{array}{l}0.1328 E-04 \\
0.8800 E-05\end{array}$ & $\begin{array}{l}0.346 \\
0.229\end{array}$ \\
\hline 196 & 4 & 1 & 146 & 1926 & 211 & -54 & 300 & $0.1115 E-04$ & 0.319 \\
\hline 215 & 4 & 3 & 36 & 2116 & 165 & 54 & $\begin{array}{l}300 \\
400\end{array}$ & $\begin{array}{l}0.68200-05 \\
0.40900-05\end{array}$ & \\
\hline 219 & 4 & 3 & 76 & 2156 & 7 & 51 & $\begin{array}{l}300 \\
400 \\
500 \\
600\end{array}$ & $\begin{array}{l}0.4559 E-05 \\
0.2523 E-05 \\
0.1432 E-05 \\
0.1092 E-05\end{array}$ & $\begin{array}{l}1.791 \\
0.991 \\
0.562 \\
0.429\end{array}$ \\
\hline 221 & 4 & 3 & 96 & 2176 & 354 & -55 & $\begin{array}{l}300 \\
400 \\
500\end{array}$ & $\begin{array}{l}0.7906 E-05 \\
0.4623 E-05 \\
0.3139 E-05\end{array}$ & $\begin{array}{l}1.212 \\
0.709 \\
0.481\end{array}$ \\
\hline 241 & 4 & 4 & 146 & 2376 & 308 & -4 & $\begin{array}{l}300 \\
400 \\
500 \\
600 \\
700\end{array}$ & $\begin{array}{l}0.5650 E-05 \\
0.3054 E-05 \\
0.2011 E-05 \\
0.1389 E-05 \\
0.1316 E-05\end{array}$ & $\begin{array}{l}2.212 \\
1.196 \\
0.787 \\
0.544 \\
0.515\end{array}$ \\
\hline 252 & 4 & 5 & 106 & 2486 & 304 & -3 & 300 & $0.2457 E-05$ & 0.419 \\
\hline 254 & 4 & 5 & 126 & 2506 & 3 & -52 & 300 & $0.5584 E-05$ & 1.113 \\
\hline 256 & 4 & 5 & 146 & 2526 & 360 & -52 & 300 & $0.4065 E-05$ & 0.628 \\
\hline 258 & 4 & 6 & 16 & 2546 & 349 & -53 & 300 & $0.5221 E-05$ & 0.668 \\
\hline 262 & 4 & 6 & 56 & 2586 & 353 & -45 & 300 & $0.4812 \mathrm{E}-05$ & 0.450 \\
\hline 263 & 4 & 6 & 66 & 2596 & 333 & -30 & 300 & $0.2324 E-05$ & 0.120 \\
\hline 264 & 4 & 6 & 76 & 2606 & 340 & -20 & $\begin{array}{l}300 \\
400\end{array}$ & $\begin{array}{l}0.3839 E-05 \\
0.1731 E-05\end{array}$ & $\begin{array}{l}0.191 \\
0.086\end{array}$ \\
\hline 265 & 4 & 6 & 86 & 2616 & 9 & -66 & $\begin{array}{l}300 \\
400\end{array}$ & $\begin{array}{l}0.5058 E-05 \\
0.3182 E-05\end{array}$ & $\begin{array}{l}0.497 \\
0.313\end{array}$ \\
\hline 266 & 4 & 6 & 96 & 2626 & 335 & -45 & 300 & $0.2418 E-05$ & 0.090 \\
\hline 267 & 4 & 6 & 106 & 2636 & 286 & -38 & 300 & $0.1486 E-05$ & 0.053 \\
\hline 268 & 4 & 6 & 116 & 2646 & 21 & -13 & $\begin{array}{l}300 \\
400\end{array}$ & $\begin{array}{l}0.2102 E-05 \\
0.7508 E-06\end{array}$ & $\begin{array}{l}0.085 \\
0.031\end{array}$ \\
\hline 269 & 4 & 6 & 126 & 2656 & 294 & -24 & 300 & $0.2051 E-06$ & 0.234 \\
\hline
\end{tabular}


Table 3. (Continued).

\begin{tabular}{|c|c|c|c|c|c|c|c|c|c|}
\hline 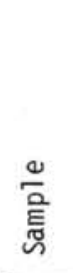 & 这 & 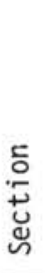 & 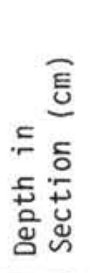 & 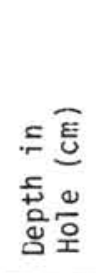 & 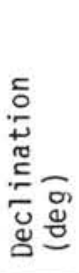 & 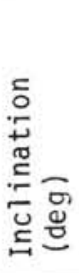 & 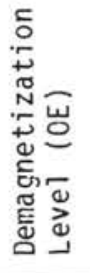 & $J(e m u)$ & $\mathrm{J}^{\mathrm{J}} / \mathrm{J}_{0}$ \\
\hline 270 & 4 & 6 & 136 & 2666 & 281 & -10 & 300 & $0.9917 E-06$ & 0.030 \\
\hline 271 & 4 & 6 & 146 & 2676 & 309 & -48 & 300 & $0.1365 E-06$ & 055 \\
\hline 272 & 4 & 7 & 6 & 2686 & 282 & -7 & 300 & $0.2052 E-05$ & 0.068 \\
\hline 273 & 4 & 7 & 16 & 2696 & 265 & -20 & 300 & $0.23105-06$ & 0.116 \\
\hline 274 & 4 & 7 & 26 & 2706 & 357 & -40 & 300 & $0.1101 E-05$ & 0.043 \\
\hline 275 & 4 & 7 & 36 & 2716 & 284 & -14 & $\begin{array}{l}300 \\
250 \\
300\end{array}$ & $\begin{array}{l}0.1790 E-05 \\
0.1656 E-06 \\
0.8989 E-06\end{array}$ & $\begin{array}{l}0.097 \\
0.088 \\
0.480\end{array}$ \\
\hline 276 & 4 & 7 & 46 & 2726 & 77 & 17 & $\begin{array}{l}300 \\
400 \\
400 \\
500 \\
500\end{array}$ & $\begin{array}{l}0.1772 E-05 \\
0.1774 E-05 \\
0.2033 E-05 \\
0.1865 E-05 \\
0.1994 E-05\end{array}$ & $\begin{array}{l}0.064 \\
0.064 \\
0.073 \\
0.067 \\
0.072\end{array}$ \\
\hline 278 & 5 & 1 & 46 & 2866 & 148 & -78 & $\begin{array}{l}300 \\
400\end{array}$ & $\begin{array}{l}0.3144 E-05 \\
0.1339 E-05\end{array}$ & $\begin{array}{l}0.341 \\
0.145\end{array}$ \\
\hline 280 & 5 & 1 & 66 & 2886 & 220 & -69 & 300 & $0.4670 E-05$ & 0.445 \\
\hline 281 & 5 & 1 & 76 & 2896 & 225 & -71 & 300 & 0 . & .898 \\
\hline 282 & 5 & 1 & 86 & 2906 & 233 & -78 & 300 & 0 . & 545 \\
\hline 284 & 5 & 1 & 106 & 2926 & 64 & 13 & 300 & 0 . & .097 \\
\hline 285 & 5 & 1 & 116 & 2936 & 71 & 6 & $\begin{array}{l}300 \\
300 \\
400\end{array}$ & $\begin{array}{l}-05 \\
-05 \\
-05\end{array}$ & $\begin{array}{l}0.090 \\
0.128 \\
0.084\end{array}$ \\
\hline 286 & 5 & 1 & 126 & 2946 & 95 & -37 & 300 & 0.77 & 0.055 \\
\hline 287 & 5 & 1 & 136 & 2956 & 87 & -4 & $\begin{array}{l}300 \\
400\end{array}$ & $\begin{array}{l}0 . \\
0 .\end{array}$ & $\begin{array}{l}0.072 \\
0.058\end{array}$ \\
\hline 288 & 5 & 1 & 146 & 2966 & 48 & 3 & 300 & 0. & 0.066 \\
\hline 302 & 5 & 5 & 130 & 3240 & 308 & -5 & $\begin{array}{l}300 \\
400\end{array}$ & 0. & $\begin{array}{l}0.107 \\
0.051\end{array}$ \\
\hline 305 & 5 & 6 & 16 & 3276 & 287 & 4 & 300 & -06 & 0.028 \\
\hline 306 & 5 & 6 & 26 & 3286 & 255 & 14 & 300 & 0 . & 64 \\
\hline 308 & 5 & 6 & 46 & 3306 & 305 & 30 & $\begin{array}{l}300 \\
400\end{array}$ & $\begin{array}{l}0.1280 E-05 \\
0.9700 E-06\end{array}$ & $\begin{array}{l}0.058 \\
0.044\end{array}$ \\
\hline 309 & 5 & 6 & 56 & 3316 & 315 & -14 & $\begin{array}{l}300 \\
400 \\
500\end{array}$ & $\begin{array}{l}0.1652 E-05 \\
0.55435-06 \\
0.1300 E-05\end{array}$ & $\begin{array}{l}0.070 \\
0.024 \\
0.055\end{array}$ \\
\hline 311 & 5 & 6 & 76 & 3336 & 294 & -22 & 300 & $0.1126 E-05$ & 0.056 \\
\hline 312 & 5 & 6 & 86 & 46 & 293 & -9 & 300 & 6 & 46 \\
\hline 313 & 5 & 6 & 96 & 3356 & 301 & 26 & 300 & $=-06$ & 0.029 \\
\hline 314 & 5 & 6 & 107 & 3367 & 265 & -9 & $\begin{array}{l}300 \\
400 \\
400\end{array}$ & $\begin{array}{l}0.6934 E-06 \\
0.3996 E-06 \\
0.7917 E-06\end{array}$ & $\begin{array}{l}0.061 \\
0.035 \\
0.070\end{array}$ \\
\hline 315 & 5 & 6 & 116 & 76 & 303 & 21 & 300 & $4 E-05$ & 0.044 \\
\hline 316 & 5 & 6 & 126 & 86 & 346 & 18 & 300 & -05 & 0.078 \\
\hline 317 & 5 & 6 & 135 & 3395 & 339 & 39 & 300 & 0.125 & 0.052 \\
\hline 321 & 5 & 7 & 26 & 3436 & 296 & -6 & 300 & 0.90038 & 0.041 \\
\hline 325 & 6 & 1 & 141 & & & & $\begin{array}{l}300 \\
400 \\
450\end{array}$ & $\begin{array}{l}0.1166 E-04 \\
0.4557 E-05 \\
0.2356 E-05\end{array}$ & $\begin{array}{l}0.187 \\
0.073 \\
0.038\end{array}$ \\
\hline 340 & 6 & 3 & 116 & 3556 & 260 & 47 & $\begin{array}{l}300 \\
400\end{array}$ & $\begin{array}{l}0.9980 E-06 \\
0.1341 E-05\end{array}$ & $\begin{array}{l}0.040 \\
0.050\end{array}$ \\
\hline 342 & 6 & 3 & 136 & 3576 & 164 & 29 & $\begin{array}{l}300 \\
400\end{array}$ & $\begin{array}{l}0.2420 F-05 \\
0.1561 E-05\end{array}$ & $\begin{array}{l}0.083 \\
0.054\end{array}$ \\
\hline 343 & 6 & 3 & 146 & 3586 & 205 & -37 & $\begin{array}{l}400 \\
500 \\
600\end{array}$ & $\begin{array}{l}0.1929 E-05 \\
0.1308 E-05 \\
0.1188 E-05\end{array}$ & $\begin{array}{l}0.094 \\
0.063 \\
0.058\end{array}$ \\
\hline 34 & 6 & 4 & 6 & 3596 & 210 & -34 & 300 & $0.3350 E-05$ & 0.172 \\
\hline 34 & 6 & 4 & 16 & 3606 & 219 & -56 & $\begin{array}{l}400 \\
500\end{array}$ & $\begin{array}{l}0.3452 E-05 \\
0.2173 E-05\end{array}$ & $\begin{array}{l}0.205 \\
0.129\end{array}$ \\
\hline
\end{tabular}


Table 3. (Continued).

\begin{tabular}{|c|c|c|c|c|c|c|c|c|c|}
\hline 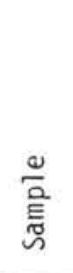 & ؛ั & 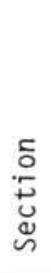 & 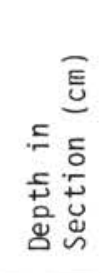 & 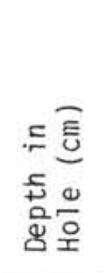 & 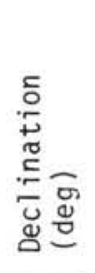 & 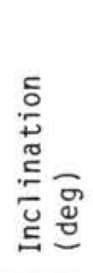 & 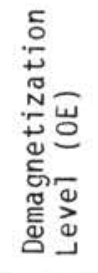 & $J(e m u)$ & $\mathrm{J} / \mathrm{J}_{0}$ \\
\hline 347 & 6 & 4 & 36 & 3626 & 46 & 8 & $\begin{array}{l}500 \\
600 \\
700 \\
800\end{array}$ & $\begin{array}{l}0.2490 E-05 \\
0.2787 E-05 \\
0.2872 E-05 \\
0.2223 E-05\end{array}$ & $\begin{array}{l}0.057 \\
0.064 \\
0.066 \\
0.051\end{array}$ \\
\hline 349 & 5 & 4 & 56 & 3646 & 106 & 13 & $\begin{array}{l}500 \\
600 \\
700\end{array}$ & $\begin{array}{l}0.2871 E-05 \\
0.2915 E-05 \\
0.2339 E-05\end{array}$ & $\begin{array}{l}0.054 \\
0.055 \\
0.044\end{array}$ \\
\hline 351 & 6 & 4 & 76 & 3666 & 47 & 49 & 300 & $0.4827 E-05$ & 0.184 \\
\hline 353 & 6 & 4 & 95 & 3686 & 50 & 11 & $\begin{array}{l}300 \\
400 \\
500 \\
600\end{array}$ & $\begin{array}{l}0.2240 E-05 \\
0.2622 E-05 \\
0.2168 E-05 \\
0.1755 E-05\end{array}$ & $\begin{array}{l}0.085 \\
0.100 \\
0.082 \\
0.067\end{array}$ \\
\hline 355 & 6 & 4 & 116 & 3706 & 245 & -50 & 300 & $0.1180 E-05$ & 0.045 \\
\hline 357 & 6 & 4 & 136 & 3726 & & & $\begin{array}{l}300 \\
400 \\
400 \\
500\end{array}$ & $\begin{array}{l}0.1233 E-05 \\
0.1212 E-05 \\
0.6656 E-06 \\
0.9008 E-06\end{array}$ & $\begin{array}{l}0.055 \\
0.054 \\
0.030 \\
0.040\end{array}$ \\
\hline 373 & 6 & 5 & 146 & 3886 & 125 & -13 & $\begin{array}{r}300 \\
400 \\
500 \\
600 \\
800 \\
1000 \\
1000\end{array}$ & $\begin{array}{l}0.1312 E-05 \\
0.1856 E-05 \\
0.3103 E-05 \\
0.3332 E-05 \\
0.3900 E-05 \\
0.4200 E-05 \\
0.4400 E-05\end{array}$ & $\begin{array}{l}0.059 \\
0.084 \\
0.141 \\
0.151 \\
0.177 \\
0.190 \\
0.199\end{array}$ \\
\hline 376 & 6 & 6 & 26 & 3916 & & & $\begin{array}{l}300 \\
300 \\
400 \\
400\end{array}$ & $\begin{array}{l}0.4524 E-06 \\
0.7400 E-06 \\
0.1510 E-05 \\
0.5800 E-06\end{array}$ & $\begin{array}{l}0.040 \\
0.066 \\
0.135 \\
0.052\end{array}$ \\
\hline 377 & 6 & 6 & 36 & 3926 & 183 & 33 & 300 & $0.9439 E-06$ & 0.048 \\
\hline 378 & 6 & 6 & 46 & 3936 & 216 & -43 & 300 & $0.7285 E-05$ & 0.204 \\
\hline 381 & 6 & 6 & 76 & 3966 & 245 & -32 & $\begin{array}{l}300 \\
400 \\
500\end{array}$ & $\begin{array}{l}0.3289 E-05 \\
0.4064 E-05 \\
0.5379 E-05\end{array}$ & $\begin{array}{l}0.259 \\
0.321 \\
0.424\end{array}$ \\
\hline 383 & 6 & 6 & 96 & 3986 & 299 & 48 & $\begin{array}{l}300 \\
400\end{array}$ & $\begin{array}{l}0.3170 E-05 \\
0.4420 E-05\end{array}$ & $\begin{array}{l}0.141 \\
0.197\end{array}$ \\
\hline 384 & 6 & 6 & 106 & 3996 & 290 & 27 & 300 & $0.3155 E-05$ & 0.101 \\
\hline 385 & 6 & 6 & 116 & 4006 & 30 & 11 & $\begin{array}{l}300 \\
400\end{array}$ & $\begin{array}{l}0.4908 E-05 \\
0.2875 E-05\end{array}$ & $\begin{array}{l}0.145 \\
0.085\end{array}$ \\
\hline 388 & 6 & 6 & 146 & 4036 & 3 & 15 & 300 & $0.3472 E-05$ & 0.127 \\
\hline 389 & 6 & 6 & 6 & 4046 & 255 & 12 & $\begin{array}{l}300 \\
400 \\
500 \\
600\end{array}$ & $\begin{array}{l}0.5214 E-05 \\
0.6814 E-05 \\
0.7657 E-05 \\
0.8285 E-05\end{array}$ & $\begin{array}{l}0.197 \\
0.257 \\
0.289 \\
0.313\end{array}$ \\
\hline 391 & 5 & 7 & 26 & 4066 & 306 & 27 & $\begin{array}{l}300 \\
400 \\
500 \\
600\end{array}$ & $\begin{array}{l}0.5510 E-05 \\
0.5310 E-05 \\
0.6320 E-05 \\
0.6830 E-05\end{array}$ & $\begin{array}{l}0.233 \\
0.224 \\
0.267 \\
0.289\end{array}$ \\
\hline 392 & 6 & 7 & 36 & 4076 & 347 & 34 & $\begin{array}{r}300 \\
400 \\
500 \\
600 \\
800 \\
1000\end{array}$ & $\begin{array}{l}0.6526 E-05 \\
0.4423 E-05 \\
0.5433 E-05 \\
0.5203 E-05 \\
0.5731 E-05 \\
0.6848 E-05\end{array}$ & $\begin{array}{l}0.208 \\
0.141 \\
0.173 \\
0.166 \\
0.182 \\
0.218\end{array}$ \\
\hline 393 & 6 & 7 & 44 & 4084 & 324 & 38 & $\begin{array}{l}300 \\
400 \\
500 \\
600 \\
700\end{array}$ & $\begin{array}{l}0.5410 E-05 \\
0.5470 E-05 \\
0.4140 E-05 \\
0.5430 E-05 \\
0.6590 E-05\end{array}$ & $\begin{array}{l}0.173 \\
0.175 \\
0.132 \\
0.173 \\
0.210\end{array}$ \\
\hline
\end{tabular}


Table 3. (Continued).

\begin{tabular}{|c|c|c|c|c|c|c|c|c|c|}
\hline$\frac{\stackrel{\varrho}{\circ}}{\text { 号 }}$ & ঠे & 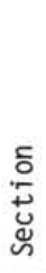 & 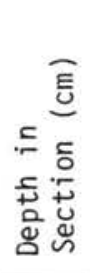 & 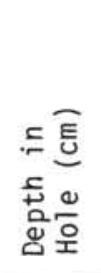 & 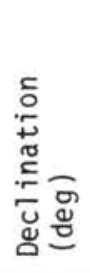 & 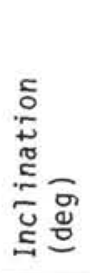 & 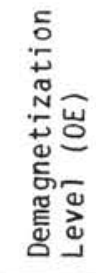 & $J$ (emu) & $J / J_{0}$ \\
\hline \multirow{4}{*}{395} & \multirow{4}{*}{7} & \multirow{4}{*}{1} & \multirow{4}{*}{16} & \multirow{4}{*}{4476} & \multirow{4}{*}{295} & \multirow{4}{*}{-39} & 800 & $0.7850 E-05$ & 0.251 \\
\hline & & & & & & & 900 & $0.5600 E-05$ & 0.179 \\
\hline & & & & & & & $\begin{array}{l}300 \\
400\end{array}$ & $\begin{array}{l}0.1054 E-04 \\
0.4661 E-05\end{array}$ & $\begin{array}{l}0.410 \\
0.181\end{array}$ \\
\hline & & & & & & & 500 & $0.3836 E-05$ & 0.149 \\
\hline \multirow[t]{6}{*}{396} & \multirow[t]{6}{*}{7} & \multirow[t]{6}{*}{1} & \multirow[t]{6}{*}{26} & \multirow[t]{6}{*}{4486} & \multirow[t]{6}{*}{357} & \multirow[t]{6}{*}{-52} & 300 & $0.2593 E-04$ & 0.292 \\
\hline & & & & & & & 400 & $0.1020 E-04$ & 0.115 \\
\hline & & & & & & & 500 & $0.6990 E-05$ & 0.079 \\
\hline & & & & & & & 600 & $0.4430 E-05$ & 0.050 \\
\hline & & & & & & & 800 & $0.3700 E-05$ & 0.042 \\
\hline & & & & & & & 1000 & $0.3980 E-05$ & 0.045 \\
\hline \multirow{4}{*}{397} & 7 & 1 & 36 & 4496 & 167 & -61 & 300 & $0.2738 E-04$ & 0.256 \\
\hline & & & & & & & 400 & $0.1313 E-04$ & 0.123 \\
\hline & & & & & & & 500 & $0.7683 E-05$ & 0.072 \\
\hline & & & & & & & 600 & $0.4709 E-05$ & 0.044 \\
\hline 398 & 7 & 1 & 46 & 4506 & 211 & -45 & 300 & $0.4884 E-04$ & 0.240 \\
\hline & & & & & & & 400 & $0.2141 E-04$ & 0.105 \\
\hline & & & & & & & 500 & $0.1352 E-04$ & 0.066 \\
\hline & & & & & & & 600 & $0.1124 E-04$ & 0.055 \\
\hline 400 & 7 & 1 & 66 & 4526 & 130 & -25 & 300 & $0.1252 E-04$ & 0.271 \\
\hline & & & & & & & 400 & $0.5118 E-05$ & 0.111 \\
\hline 405 & 7 & 1 & 116 & 4576 & 137 & -20 & 300 & $0.3051 E-05$ & 0.170 \\
\hline & & & & & & & 400 & $0.4553 E-06$ & 0.025 \\
\hline & & & & & & & 400 & $0.6295 E-06$ & 0.035 \\
\hline & & & & & & & 500 & $0.1274 E-05$ & 0.071 \\
\hline & & & & & & & 500 & $0.1924 E-05$ & 0.107 \\
\hline 408 & 7 & 1 & 146 & 4606 & 283 & 1 & 300 & $0.2743 E-05$ & 0.102 \\
\hline & & & & & & & 400 & $0.3182 E-05$ & 0.118 \\
\hline & & & & & & & 400 & $0.4367 E-05$ & 0.162 \\
\hline & & & & & & & 500 & $0.3784 E-05$ & 0.140 \\
\hline & & & & & & & 500 & $0.3832 E-05$ & 0.142 \\
\hline 410 & 7 & 2 & 26 & & & & 300 & $0.8580 E-06$ & 0.042 \\
\hline 411 & 7 & 2 & 36 & & & & 300 & $0.1439 E-05$ & 0.094 \\
\hline & & & & & & & 400 & $0.2103 E-05$ & 0.138 \\
\hline 415 & 7 & 2 & 86 & 4646 & 114 & -25 & 300 & $0.8551 E-05$ & 0.211 \\
\hline 421 & 7 & 2 & 146 & 4706 & 279 & 20 & 300 & $0.5011 \mathrm{E}-05$ & 0.119 \\
\hline & & & & & & & 400 & $0.3785 E-05$ & 0.090 \\
\hline 422 & 7 & 3 & 5 & 4716 & 258 & 23 & 300 & $0.8281 E-06$ & 0.026 \\
\hline 424 & 7 & 3 & 26 & 4736 & 242 & 12 & 150 & $0.2683 E-05$ & 0.076 \\
\hline & & & & & & & 300 & $0.1263 E-05$ & 0.036 \\
\hline & & & & & & & 300 & $0.1614 E-05$ & 0.046 \\
\hline & & & & & & & 400 & $0.1120 E-05$ & 0.032 \\
\hline & & & & & & & 400 & $0.9046 E-06$ & 0.026 \\
\hline 425 & 7 & 3 & 36 & 4746 & 159 & -23 & 300 & $0.1095 E-05$ & 0.062 \\
\hline & & & & & & & 400 & $0.2421 E-05$ & 0.138 \\
\hline & & & & & & & 400 & $0.3064 E-05$ & 0.174 \\
\hline & & & & & & & 450 & $0.2350 E-05$ & 0.134 \\
\hline & & & & & & & 500 & $0.2334 E-05$ & 0.133 \\
\hline 426 & 7 & 3 & 46 & 4756 & 223 & -18 & 300 & $0.2127 E-05$ & 0.088 \\
\hline & & & & & & & 400 & $0.1170 E-05$ & 0.048 \\
\hline & & & & & & & 500 & $0.2343 E-05$ & 0.097 \\
\hline & & & & & & & 600 & $0.1267 E-05$ & 0.052 \\
\hline & & & & & & & 600 & $0.1842 E-05$ & 0.076 \\
\hline 427 & 7 & 3 & 56 & 4766 & 286 & -23 & 400 & $0.1429 E-05$ & 0.074 \\
\hline & & & & & & & 400 & $0.9473 E-06$ & 0.049 \\
\hline & & & & & & & 500 & $0.1382 E-05$ & 0.071 \\
\hline & & & & & & & 500 & $0.1553 E-05$ & 0.080 \\
\hline 429 & 7 & 3 & 76 & 4786 & 272 & 25 & 300 & $0.2087 E-05$ & 0.074 \\
\hline
\end{tabular}


Table 3. (Continued).

\begin{tabular}{|c|c|c|c|c|c|c|c|c|c|}
\hline 寜 & ֻั & 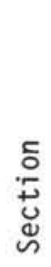 & 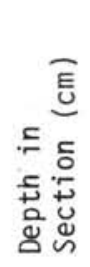 & $\begin{array}{l}\cong \bar{E} \\
\text { 들 } \\
\text { 음오 }\end{array}$ & 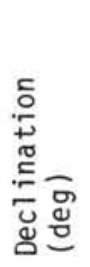 & 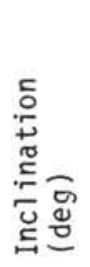 & 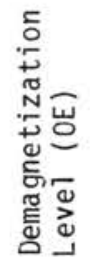 & $J(e m u)$ & $\mathrm{J} / \mathrm{J}_{0}$ \\
\hline 432 & 7 & 3 & 106 & 4816 & 305 & -40 & $\begin{array}{l}400 \\
300 \\
400 \\
500\end{array}$ & $\begin{array}{l}0.1667 E-05 \\
0.3527 E-05 \\
0.1460 E-05 \\
0.1589 E-05\end{array}$ & $\begin{array}{l}0.059 \\
0.155 \\
0.064 \\
0.070\end{array}$ \\
\hline 434 & 7 & 3 & 126 & 4836 & 283 & 31 & 300 & $0.1023 E-04$ & 0.143 \\
\hline 436 & 7 & 3 & 146 & 4856 & 275 & 36 & 300 & $0.9860 E-05$ & 0.129 \\
\hline 438 & 7 & 4 & 16 & 4876 & 266 & 34 & 300 & $0.1225 E-04$ & 0.139 \\
\hline 440 & 7 & 4 & 36 & 4896 & 278 & 40 & 300 & $0.1338 E-05$ & 0.028 \\
\hline 442 & 7 & 4 & 56 & 4916 & 251 & 49 & 300 & $0.7719 E-06$ & 021 \\
\hline 444 & 7 & 4 & 76 & 4936 & 114 & -24 & 300 & $0.5653 E-05$ & 0.181 \\
\hline 446 & 7 & 4 & 96 & 4956 & 61 & -25 & $\begin{array}{l}300 \\
400 \\
500 \\
600\end{array}$ & $\begin{array}{l}0.1366 E-05 \\
0.1862 E-05 \\
0.3275 E-05 \\
0.3413 E-05\end{array}$ & $\begin{array}{l}0.046 \\
0.063 \\
0.110 \\
0.115\end{array}$ \\
\hline 447 & 7 & 4 & 106 & 4966 & 71 & -25 & 300 & $0.25895-05$ & 0 \\
\hline 450 & 7 & 4 & 136 & 4996 & 62 & 17 & $\begin{array}{l}300 \\
300 \\
400 \\
500\end{array}$ & $\begin{array}{l}E-06 \\
E-06 \\
E-05 \\
E-05\end{array}$ & $\begin{array}{l}0.017 \\
0.026 \\
0.040 \\
0.086\end{array}$ \\
\hline $\begin{array}{l}451 \\
453\end{array}$ & $\begin{array}{l}7 \\
7\end{array}$ & $\begin{array}{l}4 \\
5\end{array}$ & $\begin{array}{r}146 \\
16\end{array}$ & $\begin{array}{l}5006 \\
5026\end{array}$ & $\begin{array}{r}91 \\
115\end{array}$ & $\begin{array}{l}-36 \\
-16\end{array}$ & $\begin{array}{l}300 \\
300\end{array}$ & $\begin{array}{l}0.4135 E-05 \\
0.9808 E-06\end{array}$ & $\begin{array}{l}0.133 \\
0.033\end{array}$ \\
\hline 459 & 7 & 5 & 76 & 5086 & 229 & 27 & $\begin{array}{l}300 \\
400\end{array}$ & $\begin{array}{l}0.2097 E-05 \\
0.1594 E-05\end{array}$ & $\begin{array}{l}0.067 \\
0.051\end{array}$ \\
\hline 460 & 7 & 5 & 86 & 5096 & 242 & 34 & 300 & $0.2234 E-05$ & 0.058 \\
\hline 46 & 7 & 5 & 96 & 5106 & 238 & 46 & $\begin{array}{l}300 \\
400 \\
500\end{array}$ & $\begin{array}{l}0.1666 E-05 \\
0.2485 E-05 \\
0.2982 E-05\end{array}$ & $\begin{array}{l}0.095 \\
0.142 \\
0.171\end{array}$ \\
\hline 465 & 7 & 5 & 136 & 5146 & 79 & -18 & $\begin{array}{l}250 \\
300\end{array}$ & $\begin{array}{l}0.6459 E-05 \\
0.4089 E-05\end{array}$ & $\begin{array}{l}0.366 \\
0.232\end{array}$ \\
\hline 466 & 7 & 5 & 146 & 5156 & 100 & -40 & $\begin{array}{l}300 \\
400 \\
400 \\
500\end{array}$ & $\begin{array}{l}0.1431 E-05 \\
0.2179 E-05 \\
0.6568 E-06 \\
0.1013 E-05\end{array}$ & $\begin{array}{l}0.053 \\
0.080 \\
0.024 \\
0.037\end{array}$ \\
\hline 469 & 7 & 6 & 26 & 5186 & 119 & -17 & 300 & $0.1176 E-05$ & 0.034 \\
\hline 471 & 7 & 6 & 46 & 5206 & 102 & 8 & $\begin{array}{l}250 \\
300\end{array}$ & $\begin{array}{l}0.1576 E-05 \\
0.4906 E-06\end{array}$ & $\begin{array}{l}0.049 \\
0.015\end{array}$ \\
\hline 472 & 7 & 6 & 56 & 5216 & 94 & -28 & 300 & $0.3531 E-05$ & 0.169 \\
\hline & 7 & 6 & 86 & 5246 & & & $\begin{array}{l}300 \\
400 \\
500\end{array}$ & $\begin{array}{l}0.1829 E-05 \\
0.2760 E-05 \\
0.2992 E-05\end{array}$ & $\begin{array}{l}0.053 \\
0.079 \\
0.086\end{array}$ \\
\hline 477 & 7 & 6 & 106 & 5266 & 283 & 35 & $\begin{array}{l}300 \\
400\end{array}$ & $\begin{array}{l}0.2785 E-05 \\
0.2138 E-05\end{array}$ & $\begin{array}{l}0.065 \\
0.050\end{array}$ \\
\hline 479 & 7 & 6 & 126 & 5286 & 48 & -19 & 300 & $0.1420 E-05$ & 0.040 \\
\hline 480 & 7 & 6 & 136 & 5296 & 94 & -29 & $\begin{array}{l}300 \\
400 \\
500\end{array}$ & $\begin{array}{l}0.3077 E-05 \\
0.8370 E-06 \\
0.2507 E-05\end{array}$ & $\begin{array}{l}0.143 \\
0.039 \\
0.116\end{array}$ \\
\hline 481 & 7 & 6 & 146 & 5306 & 108 & 15 & $\begin{array}{l}300 \\
400\end{array}$ & $\begin{array}{l}0.1290 E-04 \\
0.4970 E-05\end{array}$ & $\begin{array}{l}0.374 \\
0.144\end{array}$ \\
\hline 482 & 7 & 7 & 6 & 5316 & 100 & 21 & $\begin{array}{l}300 \\
400\end{array}$ & $\begin{array}{l}0.6480 E-05 \\
0.2221 E-05\end{array}$ & $\begin{array}{l}0.306 \\
0.105\end{array}$ \\
\hline 483 & 7 & 7 & 16 & 5326 & 127 & 26 & $\begin{array}{l}300 \\
400 \\
400\end{array}$ & $\begin{array}{l}0.1830 E-05 \\
0.1830 E-05 \\
0.2130 E-05\end{array}$ & $\begin{array}{l}0.048 \\
0.048 \\
0.056\end{array}$ \\
\hline 484 & 7 & 7 & 26 & 5336 & 293 & 51 & $\begin{array}{l}300 \\
300\end{array}$ & $\begin{array}{l}0.1230 E-05 \\
0.9400 E-06\end{array}$ & $\begin{array}{l}0.030 \\
0.023\end{array}$ \\
\hline 485 & 7 & 7 & 36 & 5346 & 259 & 45 & $\begin{array}{l}300 \\
400 \\
500\end{array}$ & $\begin{array}{l}0.2310 E-05 \\
0.1940 E-05 \\
0.2960 E-05\end{array}$ & $\begin{array}{l}0.058 \\
0.049 \\
0.075\end{array}$ \\
\hline
\end{tabular}


Table 3. (Continued).

\begin{tabular}{|c|c|c|c|c|c|c|c|c|c|}
\hline 总 & ذِ & 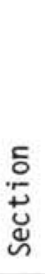 & 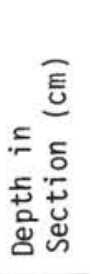 & 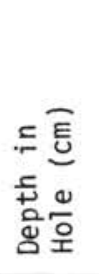 & 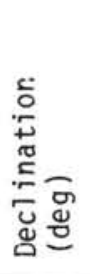 & 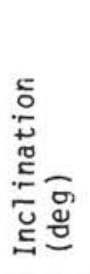 & 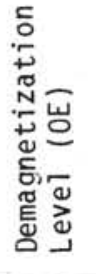 & J (emu) & $3 /]_{0}$ \\
\hline & & & & & & & $\begin{array}{l}600 \\
800\end{array}$ & $\begin{array}{l}0.2810 E-05 \\
0.3370 E-05\end{array}$ & $\begin{array}{l}0.071 \\
0.085\end{array}$ \\
\hline & & & & & & & 1000 & $0.3340 E-05$ & 0.084 \\
\hline & & & & & & & 1000 & $0.3510 \mathrm{E}-$ & 0.089 \\
\hline \multirow[t]{5}{*}{487} & 8 & 1 & 26 & 5436 & 146 & -51 & 300 & $0.1048 E-04$ & 0.498 \\
\hline & & & & & & & 400 & $0.3773 E-05$ & 0.179 \\
\hline & & & & & & & 500 & 0 . & 0.171 \\
\hline & & & & & & & 500 & 5 & 0.182 \\
\hline & & & & & & & 600 & 5 & 0.178 \\
\hline \multirow[t]{3}{*}{488} & 8 & 1 & 36 & 5446 & 118 & -67 & 300 & $0.6513 E-05$ & 0.200 \\
\hline & & & & & & & 400 & 0. & 0.132 \\
\hline & & & & & & & 500 & $0.4006 E-05$ & 0.123 \\
\hline \multirow[t]{4}{*}{489} & 8 & 1 & 46 & 5456 & 143 & -18 & 300 & $0.1440 E-04$ & 0.193 \\
\hline & & & & & & & 400 & $0.7524 \varepsilon-05$ & 0.101 \\
\hline & & & & & & & 500 & $0.4269 E-05$ & 0.057 \\
\hline & & & & & & & 600 & $0.3166 E-05$ & 0.043 \\
\hline \multirow[t]{3}{*}{490} & 8 & 1 & 56 & 5466 & 318 & -53 & 300 & $0.5509 E-05$ & 0.193 \\
\hline & & & & & & & 400 & $0.1884 E-05$ & 0.066 \\
\hline & & & & & & & 500 & $0.2255 E-05$ & 0.079 \\
\hline 491 & 8 & 1 & 66 & 5476 & 312 & -41 & 300 & $0.5083 E-05$ & 0.162 \\
\hline \multirow[t]{2}{*}{494} & 8 & 1 & 96 & 5506 & 190 & 1 & 300 & $0.26425-05$ & 0.030 \\
\hline & & & & & & & 300 & $0.2751 E-05$ & 0.032 \\
\hline 495 & 8 & 1 & 106 & 5516 & 327 & -13 & 300 & $0.9768 E-05$ & 0.298 \\
\hline \multirow[t]{2}{*}{498} & 8 & 1 & 136 & 5546 & 190 & -3 & 300 & $0.4784 E-05$ & 0.073 \\
\hline & & & & & & & 400 & $0.1724 E-05$ & 0.026 \\
\hline 499 & 8 & 1 & 146 & 5556 & 321 & -20 & 300 & $0.9775 E-05$ & 0.327 \\
\hline \multirow[t]{4}{*}{500} & 8 & 2 & 5 & 5565 & 137 & 15 & 300 & $0.2161 E-04$ & 0.222 \\
\hline & & & & & & & 400 & $0.10645-04$ & 0.109 \\
\hline & & & & & & & 500 & $E-05$ & 0.049 \\
\hline & & & & & & & 500 & $E-05$ & 0.049 \\
\hline \multirow[t]{2}{*}{502} & 8 & 2 & 32 & 5592 & 141 & 17 & 300 & $0.1069 E-04$ & $\begin{array}{l}0.142 \\
0.089\end{array}$ \\
\hline & & & & & & & 500 & $0.2200 E-05$ & 0.029 \\
\hline 503 & 8 & 2 & 67 & 5627 & 178 & 49 & 300 & $0.2262 E-05$ & 0.046 \\
\hline 505 & 8 & 2 & 106 & 5666 & 140 & 19 & 300 & $0.1180 E-04$ & 0.164 \\
\hline \multirow[t]{2}{*}{507} & 8 & 2 & 126 & 5686 & 292 & -9 & 300 & $0.3371 \mathrm{E}-05$ & 0.062 \\
\hline & & & & & & & 400 & 0 & 0.022 \\
\hline \multirow[t]{2}{*}{509} & 8 & 2 & 146 & 5706 & 304 & 54 & 300 & $0.1592 E-05$ & 0.041 \\
\hline & & & & & & & 400 & $0.8995 E-$ & 0.023 \\
\hline 511 & 8 & 3 & 66 & 5776 & 123 & -20 & 300 & $0.1494 E-05$ & 0.039 \\
\hline \multirow[t]{2}{*}{513} & 8 & 3 & 101 & 5811 & 146 & 18 & 250 & $0.2269 E-05$ & 0.044 \\
\hline & & & & & & & 300 & $0.1811 \mathrm{E}-$ & 0.035 \\
\hline \multirow[t]{2}{*}{514} & 8 & 3 & 147 & 5857 & 311 & -14 & 300 & $0.4948 E-05$ & 0.158 \\
\hline & & & & & & & 400 & 0.106 & 0.034 \\
\hline \multirow[t]{2}{*}{515} & 8 & 4 & 3 & 5863 & 132 & -18 & 300 & $0.6623 E-05$ & 0.340 \\
\hline & 8 & 4 & 23 & 5883 & 114 & & 40 & & $\begin{array}{l}0.121 \\
0.137\end{array}$ \\
\hline 517 & 0 & 4 & 23 & 5883 & 4 & 9 & $\begin{array}{l}500 \\
400\end{array}$ & $0.2093 E-05$ & 0.084 \\
\hline \multirow[t]{4}{*}{521} & 8 & 6 & 77 & 6237 & & & 300 & $0.2245 E-04$ & 1.029 \\
\hline & & & & & & & 40 & $E-05$ & 9 \\
\hline & & & & & & & 500 & $0.4326 E-05$ & 0.198 \\
\hline & & & & & & & 50 & 0 . & 0.199 \\
\hline \multirow[t]{2}{*}{522} & 8 & 6 & 90 & 6250 & 345 & -14 & 300 & $0.2051 E-04$ & 0.643 \\
\hline & 8 & 7 & & & & & 400 & $0.8676 E-05$ & 0.272 \\
\hline & 0 & 1 & 3. & & 157 & -12 & 300 & $0.9922 E-06$ & 0.044 \\
\hline
\end{tabular}


Table 4. Magnetization of Hole 576B samples after AF demagnetization at intensities above $100 \mathrm{Oe}$.

\begin{tabular}{|c|c|c|c|c|c|c|c|}
\hline 怘 & ذّ & 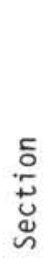 & 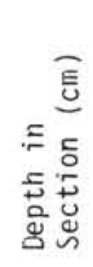 & 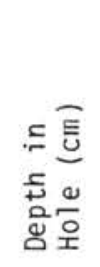 & 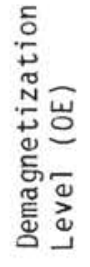 & J (emu) & $\mathrm{J} / \mathrm{J}_{0}$ \\
\hline 617 & 1 & 5 & 6 & 606 & 300 & $0.1255 E-04$ & 0.555 \\
\hline 624 & 1 & 5 & 76 & 676 & 201 & $0.1309 E-04$ & 0.668 \\
\hline 625 & 1 & 5 & 86 & $\begin{array}{l}686 \\
686 \\
686 \\
686 \\
686\end{array}$ & $\begin{array}{l}300 \\
301 \\
400 \\
500 \\
600\end{array}$ & $\begin{array}{l}0.6085 E-05 \\
0.4694 E-05 \\
0.4220 E-05 \\
0.3285 E-05 \\
0.2845 E-05\end{array}$ & $\begin{array}{l}0.861 \\
0.664 \\
0.597 \\
0.465 \\
0.402\end{array}$ \\
\hline 611 & 1 & 5 & 6 & 756 & 300 & $0.9197 E-05$ & 0.739 \\
\hline 632 & 2 & 1 & 11 & 1171 & 201 & $0.9128 E-05$ & 0.846 \\
\hline 635 & 2 & 1 & 36 & 1196 & 201 & $0.1590 E-04$ & 0.747 \\
\hline 685 & 2 & 5 & 16 & 1776 & 300 & $0.3081 E-05$ & 0.831 \\
\hline 688 & 2 & 5 & 46 & 1806 & 300 & $0.8236 E-05$ & 0.608 \\
\hline 707 & 2 & 6 & 86 & 1996 & 201 & $0.4483 E-05$ & 1.257 \\
\hline 711 & 2 & 6 & 126 & $\begin{array}{l}2036 \\
2036\end{array}$ & $\begin{array}{l}202 \\
300\end{array}$ & $\begin{array}{l}0.5460 E-06 \\
0.5960 E-06\end{array}$ & $\begin{array}{l}0.073 \\
0.080\end{array}$ \\
\hline 713 & 2 & 6 & 146 & 2056 & 300 & $0.4284 E-06$ & 0.073 \\
\hline 714 & 2 & 7 & 6 & $\begin{array}{l}2066 \\
2066 \\
2066 \\
2066 \\
2066\end{array}$ & $\begin{array}{l}201 \\
300 \\
400 \\
500 \\
600\end{array}$ & $\begin{array}{l}0.6357 E-05 \\
0.4061 E-05 \\
0.2057 E-05 \\
0.1327 E-05 \\
0.1174 E-05\end{array}$ & $\begin{array}{l}0.596 \\
0.381 \\
0.193 \\
0.125 \\
0.110\end{array}$ \\
\hline 716 & 2 & 7 & 26 & $\begin{array}{l}2086 \\
2086 \\
2086 \\
2086 \\
2086 \\
2086\end{array}$ & $\begin{array}{l}300 \\
301 \\
400 \\
500 \\
600 \\
700\end{array}$ & $\begin{array}{l}0.4193 E-05 \\
0.5282 E-05 \\
0.2936 E-05 \\
0.1678 E-05 \\
0.1240 E-05 \\
0.7874 E-06\end{array}$ & $\begin{array}{l}0.294 \\
0.371 \\
0.206 \\
0.118 \\
0.087 \\
0.055\end{array}$ \\
\hline 752 & 3 & 3 & 46 & $\begin{array}{l}2326 \\
2326\end{array}$ & $\begin{array}{l}300 \\
400\end{array}$ & $\begin{array}{l}0.2409 E-05 \\
0.15475-05\end{array}$ & $\begin{array}{l}0.249 \\
0.160\end{array}$ \\
\hline 770 & 3 & 6 & 126 & $\begin{array}{l}2552 \\
2552\end{array}$ & $\begin{array}{l}300 \\
400\end{array}$ & $\begin{array}{l}0.6193 E-06 \\
0.3523 E-06\end{array}$ & $\begin{array}{l}0.039 \\
0.022\end{array}$ \\
\hline 775 & 4 & 3 & 106 & $\begin{array}{l}3416 \\
3416\end{array}$ & $\begin{array}{l}300 \\
400\end{array}$ & $\begin{array}{l}0.1801 E-05 \\
0.9116 E-06\end{array}$ & $\begin{array}{l}0.122 \\
0.062\end{array}$ \\
\hline 776 & 4 & 3 & 126 & 3436 & 300 & $0.16725-05$ & 0.189 \\
\hline 777 & $\begin{array}{l}4 \\
4\end{array}$ & 3 & $\begin{array}{r}146 \\
6\end{array}$ & $\begin{array}{l}3456 \\
3466\end{array}$ & 300 & $0.46225-06$ & $\begin{array}{l}0.034 \\
0.060\end{array}$ \\
\hline $\begin{array}{l}778 \\
779\end{array}$ & $\begin{array}{l}4 \\
4\end{array}$ & $\begin{array}{l}4 \\
4\end{array}$ & $\begin{array}{r}6 \\
26\end{array}$ & $\begin{array}{l}3466 \\
3486\end{array}$ & $\begin{array}{l}300 \\
300\end{array}$ & $\begin{array}{l}0.1027 E-05 \\
0.7908 E-06\end{array}$ & $\begin{array}{l}0.060 \\
0.055\end{array}$ \\
\hline 780 & 4 & 4 & 46 & 3506 & 300 & $0.7645 E-06$ & 0.050 \\
\hline 781 & 4 & 4 & 66 & 3526 & 300 & $0.7230 E-06$ & 0.060 \\
\hline 782 & 4 & 4 & 86 & 3546 & 300 & $0.7836 \tilde{z}-06$ & 0.064 \\
\hline 783 & 4 & 4 & 106 & 3566 & 300 & $0.8954 E-06$ & 0.062 \\
\hline 785 & 4 & 4 & 146 & 3606 & 300 & $0.7728 E-06$ & 0.052 \\
\hline 786 & 4 & 5 & 6 & $\begin{array}{l}3616 \\
3615\end{array}$ & $\begin{array}{l}300 \\
401\end{array}$ & $\begin{array}{l}0.14455-05 \\
0.6454 E-06\end{array}$ & $\begin{array}{l}0.099 \\
0.044\end{array}$ \\
\hline 787 & 4 & 5 & 26 & 3636 & 300 & $0.6849 \equiv-06$ & 0.045 \\
\hline 788 & 4 & 5 & 46 & 3656 & 300 & $0.8751 E-06$ & 0.043 \\
\hline 789 & 4 & 5 & 66 & 3676 & 300 & $0.9333 E-06$ & 0.058 \\
\hline 790 & 4 & 5 & 86 & 3696 & 300 & $0.1566 E-05$ & 0.095 \\
\hline 791 & 4 & 5 & 106 & 3716 & 300 & $0.2116 E-05$ & 0.133 \\
\hline 792 & 4 & 5 & 126 & $\begin{array}{l}3736 \\
3736\end{array}$ & $\begin{array}{l}300 \\
400\end{array}$ & $\begin{array}{l}0.1622 E-05 \\
0.9335 E-06\end{array}$ & $\begin{array}{l}0.000 \\
0.000\end{array}$ \\
\hline 793 & 4 & 5 & 146 & $\begin{array}{l}3756 \\
3756\end{array}$ & $\begin{array}{l}300 \\
400\end{array}$ & $\begin{array}{l}0.1806 E-05 \\
0.83065-06\end{array}$ & $\begin{array}{l}0.179 \\
0.082\end{array}$ \\
\hline 794 & 4 & 6 & 6 & 3766 & 300 & $0.1663 E-05$ & 0.159 \\
\hline 795 & 4 & 6 & 26 & 3786 & 300 & $0.1433 E-05$ & 0.165 \\
\hline 795 & 4 & 6 & 46 & 3806 & 300 & $0.2593 E-05$ & 0.311 \\
\hline 800 & 4 & 6 & 126 & $\begin{array}{l}3806 \\
3886\end{array}$ & $\begin{array}{l}400 \\
300\end{array}$ & $\begin{array}{l}0.1095 E-05 \\
0.3337 E-05\end{array}$ & $\begin{array}{l}0.132 \\
0.268\end{array}$ \\
\hline
\end{tabular}


Table 4. (Continued).

\begin{tabular}{|c|c|c|c|c|c|c|c|}
\hline 高 & 엉 & 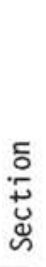 & 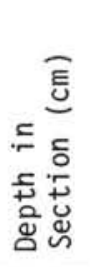 & $\begin{array}{l}\approx \widehat{E} \\
\text { 흘 } \\
\text { 웜우 }\end{array}$ & 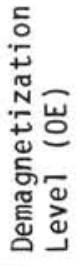 & J (emu) & $\mathrm{J} / \mathrm{J}_{0}$ \\
\hline \multirow[t]{5}{*}{801} & 4 & 6 & 146 & 3906 & 300 & $0.5924 \subseteq-05$ & 0.183 \\
\hline & & & & 3906 & 400 & $0.3753 E-05$ & 0.116 \\
\hline & & & & 3906 & 500 & $0.3010 E-05$ & 0.093 \\
\hline & & & & 3906 & 600 & $0.3041 E-05$ & 0.094 \\
\hline & & & & 3906 & 700 & $0.2625 E-05$ & 0.081 \\
\hline 806 & 5 & 2 & 5 & 4016 & 300 & $0.30725-05$ & 0.188 \\
\hline 809 & 5 & 2 & 57 & 4077 & 300 & $0.1872 \varepsilon-05$ & 0.080 \\
\hline 810 & 5 & 2 & 87 & 4097 & 300 & $0.1948 \subseteq-05$ & 0.086 \\
\hline \multirow[t]{2}{*}{817} & 5 & 3 & 66 & 4226 & 300 & $0.7327 E-06$ & 0.041 \\
\hline & & & & 4226 & 301 & $0.1094 \Xi-05$ & 0.061 \\
\hline 818 & 5 & 3 & 86 & 4246 & 300 & $0.2446 E-05$ & 0.151 \\
\hline 820 & 5 & 3 & 125 & 4286 & 201 & $0.5567 E-05$ & 0.345 \\
\hline 821 & 5 & 3 & 146 & 4306 & 201 & $0.1076 \subseteq-04$ & 0.688 \\
\hline 835 & 5 & 5 & 107 & 4567 & 300 & $0.5734 E-05$ & 0.116 \\
\hline \multirow[t]{2}{*}{836} & 5 & 5 & 126 & 4586 & 300 & $0.3053 E-05$ & 0.241 \\
\hline & & & & 4586 & 400 & $0.40375-05$ & 0.318 \\
\hline 838 & 5 & 6 & 6 & 4616 & 300 & $0.7755 E-05$ & 0.512 \\
\hline 839 & 5 & 6 & 26 & 4636 & 300 & $0.9557 E-05$ & 0.523 \\
\hline 850 & 6 & 2 & 86 & 5242 & 300 & $0.1668 \varepsilon-05$ & 0.045 \\
\hline 852 & 6 & 2 & 126 & 5282 & 300 & $0.1843 E-05$ & 0.048 \\
\hline 865 & 6 & 4 & 66 & 5512 & 300 & $0.1478 E-05$ & 0.039 \\
\hline \multirow[t]{3}{*}{874} & 6 & 6 & 47 & 5793 & 300 & $0.3731 E-05$ & 0.091 \\
\hline & & & & 5793 & 400 & $0.2211 \mathrm{E}-05$ & 0.054 \\
\hline & & & & 5793 & 500 & $0.23325-05$ & 0.057 \\
\hline 893 & 8 & 5 & 142 & 7487 & 300 & $0.3206 E-05$ & 0.273 \\
\hline 894 & 8 & 6 & 44 & 7539 & 300 & $0.5708 E-05$ & 0.423 \\
\hline
\end{tabular}

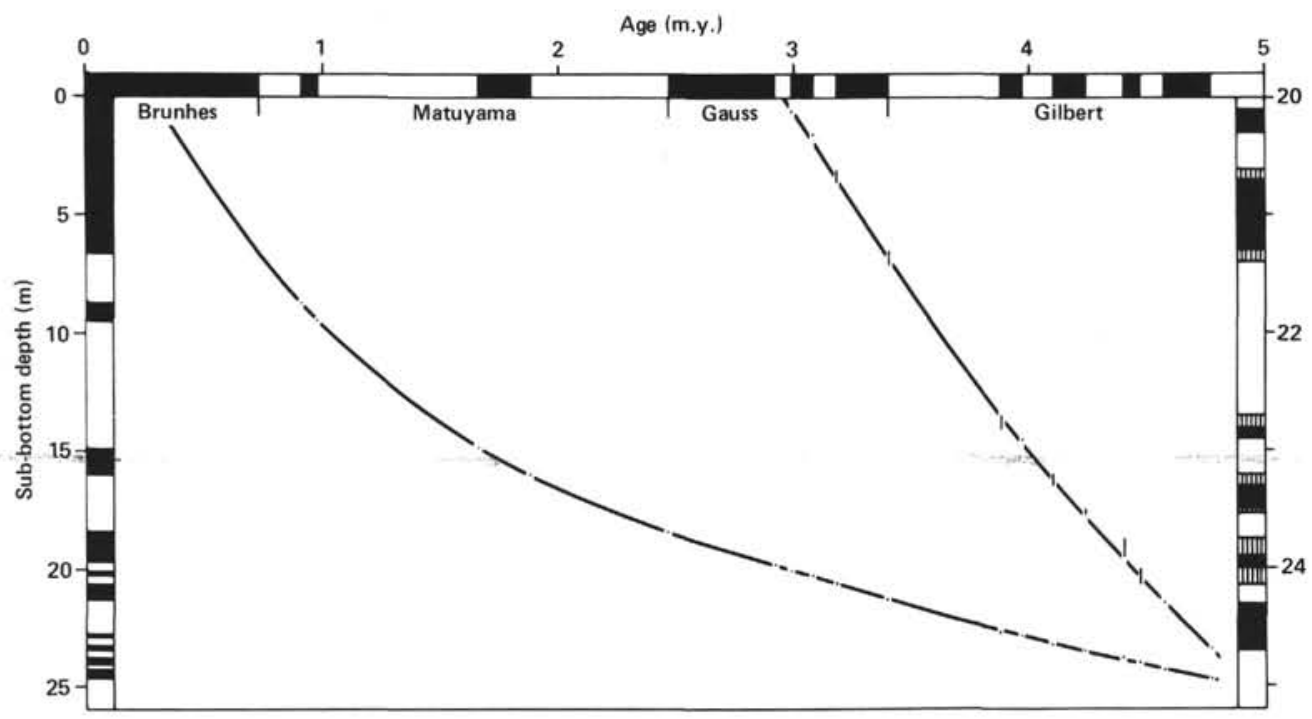

Figure 2. Correlation of the paleomagnetic polarity stratigraphy of Site 576 with the time scale of Berggren et al. (in press). The Gauss-Gilbert interval is replotted at an enlarged vertical scale (right-hand curve and depth scale; partly filled bars show intervals of uncertain polarity). 
Table 5. Magnetostratigraphy for Site 576.

\begin{tabular}{lrcl}
\hline & \multicolumn{2}{c}{ Depth (m) } & \\
\cline { 2 - 3 } $\begin{array}{c}\text { Age } \\
(\mathrm{m} . \mathrm{y} .)\end{array}$ & Hole 576 & Hole 576B & Boundary or event \\
\hline 0.73 & $6.61 \pm 0.05$ & $6.61 \pm 0.04$ & Brunhes/Matuyama \\
0.91 & $8.76 \pm 0.04$ & \pm & Jaramillo \\
0.98 & $9.51 \pm 0.09$ & - & \\
1.66 & $14.91 \pm 0.04$ & $14.91 \pm 0.04$ & Olduvai \\
1.88 & $16.01 \pm 0.04$ & - & Matuyama/Gauss \\
2.47 & - & $18.41 \pm 0.04$ & Kana \\
2.92 & $19.81 \pm 0.04$ & $19.91 \pm 0.04$ & Kaena \\
2.99 & $20.11 \pm 0.04$ & $20.11 \pm 0.04$ & \\
3.08 & $20.31 \pm 0.04$ & $20.31 \pm 0.04$ & Mammoth \\
3.18 & $20.71 \pm 0.04$ & $20.61 \pm 0.04$ & \\
3.40 & $21.41 \pm 0.04$ & $21.31 \pm 0.04$ & Gauss/Gilbert \\
3.88 & $22.71 \pm 0.04$ & $22.81 \pm 0.04$ & Cochiti \\
3.97 & $22.91 \pm 0.04$ & $22.91 \pm 0.04$ & \\
4.10 & $23.31 \pm 0.04$ & \pm & Nunivak \\
4.24 & $23.51 \pm 0.04$ & - & \\
4.40 & $23.91 \pm 0.04$ & - & Sidufjall \\
4.47 & $24.01 \pm 0.04$ & \pm & \\
4.57 & $24.31 \pm 0.04$ & - & Thvera \\
4.77 & $24.71 \pm 0.04$ & $24.61 \pm 0.04$ & \\
\hline
\end{tabular}

Note: - means not recovered.

${ }^{\mathrm{a}}$ From Berggren et al. (in press).

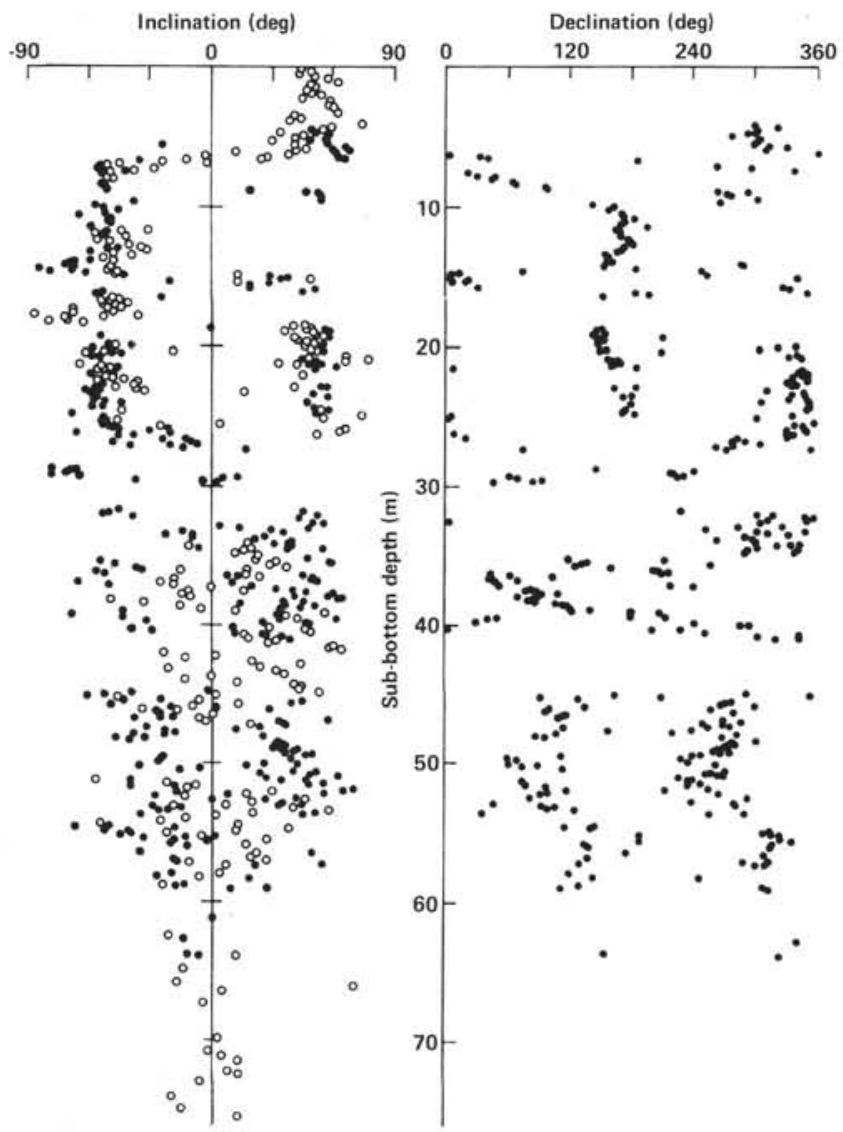

Figure 3. Inclination (filled circles, Hole 576; open circles, Hole 576B) and declination (Hole 576 only) of magnetic samples as a function of depth at Site 576.

tories at the two sites from the middle Miocene through the Pliocene (Fig. 11).

Prior to 16 m.y. ago at Site 578 and 5 m.y. at Site 576 , the age-depth curves are constrained only by ich- thyolith (see Doyle and Riedel, this volume) and limited foraminiferal (see D'Agostino, this volume) stratigraphy. The uncertainties in age assignments and relatively wide spacings of the control points conceal any short-term rate changes (i.e., $<5-10 \mathrm{~m}$.y.).

Within the uncertainties of the initial ichthyolith stratigraphies, the accumulation rates at the two sites from 16 to 70 m.y. ago are identical and uniform at about $0.4 \mathrm{~m} / \mathrm{m} . \mathrm{y}$.

From about 16 to 2 m.y. ago, sediment accumulated at Site 578 about five times as fast as at Site 576. The process responsible for this difference has not been identified, but the sharpness of the isopach gradient near Site 578 (see Jacobi et al., this volume) suggests that transport by bottom currents to this site, rather than introduction of excess sediment at the sea surface, is a likely explanation.

\section{CONCLUSIONS}

Sites 576 and 578 both appear to contain essentially complete Cenozoic sections of pelagic clay. At Site 576, the accumulation rate increases gradually from about $0.4 \mathrm{~m} / \mathrm{m}$.y. prior to $15 \mathrm{~m}$.y. ago to about $4 \mathrm{~m} / \mathrm{m}$.y. at 2 m.y. ago. An influx of Pleistocene eolian debris then results in a rapid increase to a rate in excess of $15 \mathrm{~m} /$ m.y. today. Stable detrital remanence yields a good paleomagnetic record for the past $5 \mathrm{~m}$.y., during which period the accumulation rate has exceeded $2 \mathrm{~m} / \mathrm{m}$.y.

At Site 578, the accumulation rate prior to $16 \mathrm{~m} . \mathrm{y}$. ago was $0.3-0.4 \mathrm{~m} / \mathrm{m}$.y. It then increased abruptly to 2-4 m/m.y. for the interval from 16 to $9 \mathrm{~m}$.y. ago. Following a hiatus from 8.2 to $8.8 \mathrm{~m} . \mathrm{y}$. ago, the rate increased fairly uniformly to the surface, reaching a maximum value of about $38 \mathrm{~m} / \mathrm{m}$.y. The rate increase during the Quaternary is very similar to the pattern at Site $\mathbf{5 7 6 .}$

Site 578 contains a remarkable record of detrital remanence spanning the past $16 \mathrm{~m}$.y. About 60 reversals can be correlated to the standard paleomagnetic stratigraphic section, yielding a detailed age-depth curve for the site. As at Site 576, the magnetic stratigraphy breaks down when the accumulation rate drops below about $2 \mathrm{~m} / \mathrm{m}$.y., owing, apparently, to "swamping" of the detrital remanence by the unstable chemical remanence of authigenic oxyhydroxides.

\section{ACKNOWLEDGMENTS}

We are grateful to the members of the scientific party of Leg 86 for many stimulating discussions. Bob Karlin, Dennis Schultz, Dave Rea, Carlos Lopez, Chris Dymond, and Dick Kovar helped enter, plot, and cross-check data. Bob Karlin designed the sampler that worked so well at sea, and built the mu-metal boxes. Ronald Merrill and Dennis Kent reviewed this manuscript. To all these people, our sincere thanks.

Support for the shore-based studies came from Sandia National Laboratories Contract SAN26-6611.

\section{REFERENCES}

Barton, C., and Sopher, C., 1982. Paleomagnetic study of areas B1, $\mathrm{C} 1$ and E3. In Hinga, K. R. (Ed.), Subseabed Disposal Program Annual Report January to September 1981 (Vol. II, App. L): Albuquerque, NM (Sandia National Laboratories), Rept. SAND820664/II, 235-334.

Berggren, W. A., Kent, D. V., and Flynn, J. J., in press. Paleogene geochronology and chronostratigraphy. In Snelling, N. J. (Ed.), 
Geochronology and the Geological Record. Spec. Publ. Geol. Soc. London.

Chase, T. E., 1975. Topography of the oceans. Univ. Calif. San Diego, Scripps Inst. Oceanog., Inst. Mar. Resources Tech. Rept., Ser. TR57.

Kent, D. V., and Lowrie, W., 1974. Origin of magnetic instability in sediment cores from the central North Pacific. J. Geophys. Res., 79:2987-3000.

Leinen, M., and Heath, G. R., 1981. Sedimentary indicators of atmospheric circulation in the northern hemisphere during the Cenozoic. Palaeogeogr., Palaeoclimat., Palaeoecol., 36:1-21.

McDougall, I., 1977. The present status of the geomagnetic polarity time scale. Publ. Aust. Natl. Univ., Res. Sch. Earth Sci., 1288.

Moore, T. C., Jr., and Heath, G. R., 1978. Sea-floor sampling techniques. In Riley, J. P., and Chester, R. (Eds.), Chemical Oceanography (Vol. 7): New York (Academic Press), 75-126.
Ness, G., Levi, S., and Couch, R., 1980. Marine magnetic anomaly timescales for the Cenozoic and Late Cretaceous: a precis, critique, and synthesis. Rev. Geophys. Space Phys., 18:753-770.

Opdyke, N. D., and Foster, J. H., 1970. Paleomagnetism of cores from the North Pacific. In Hays, J. D. (Ed.), Geological Investigations of the North Pacific. Mem. Geol. Soc. Am., 126:83-119.

Prince, R. A., Heath, G. R., and Kominz, M., 1980. Paleomagnetic studies of central North Pacific sediment cores: stratigraphy, sedimentation rates, and the origin of magnetic instability. Geol. Soc. Am. Bull., 91(II):1789-1835.

Date of Initial Receipt: 7 November 1984 Date of Acceptance: 14 June 1984
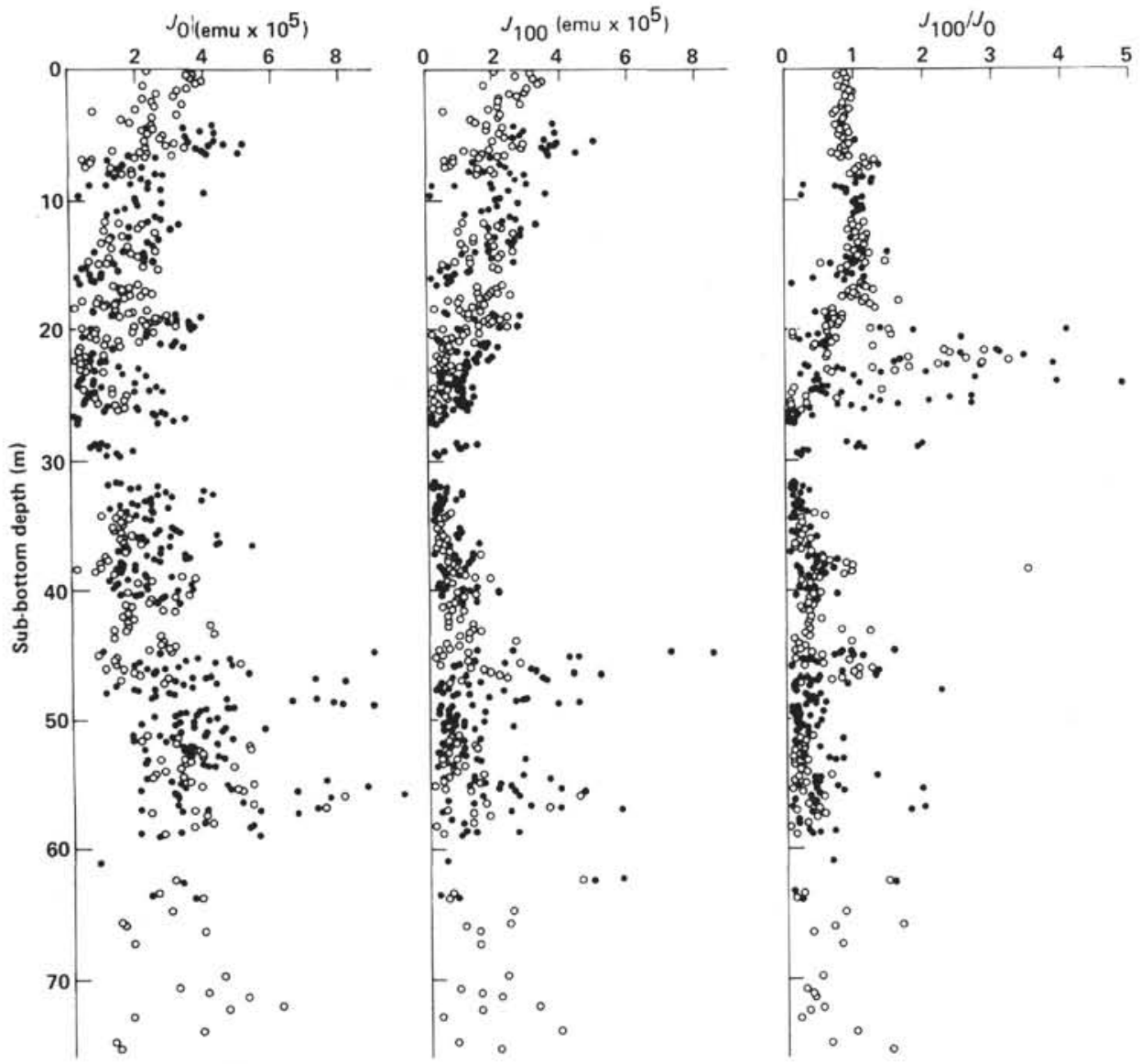

Figure 4. Natural remanent magnetization $\left(J_{0}\right)$, remanent intensity after AF demagnetization at $100 \mathrm{Oe}$ $\left(J_{100}\right)$, and $J_{100} / J_{0}$ for samples from Site 576. Filled circles, Hole 576; open circles, Hole 576B. 


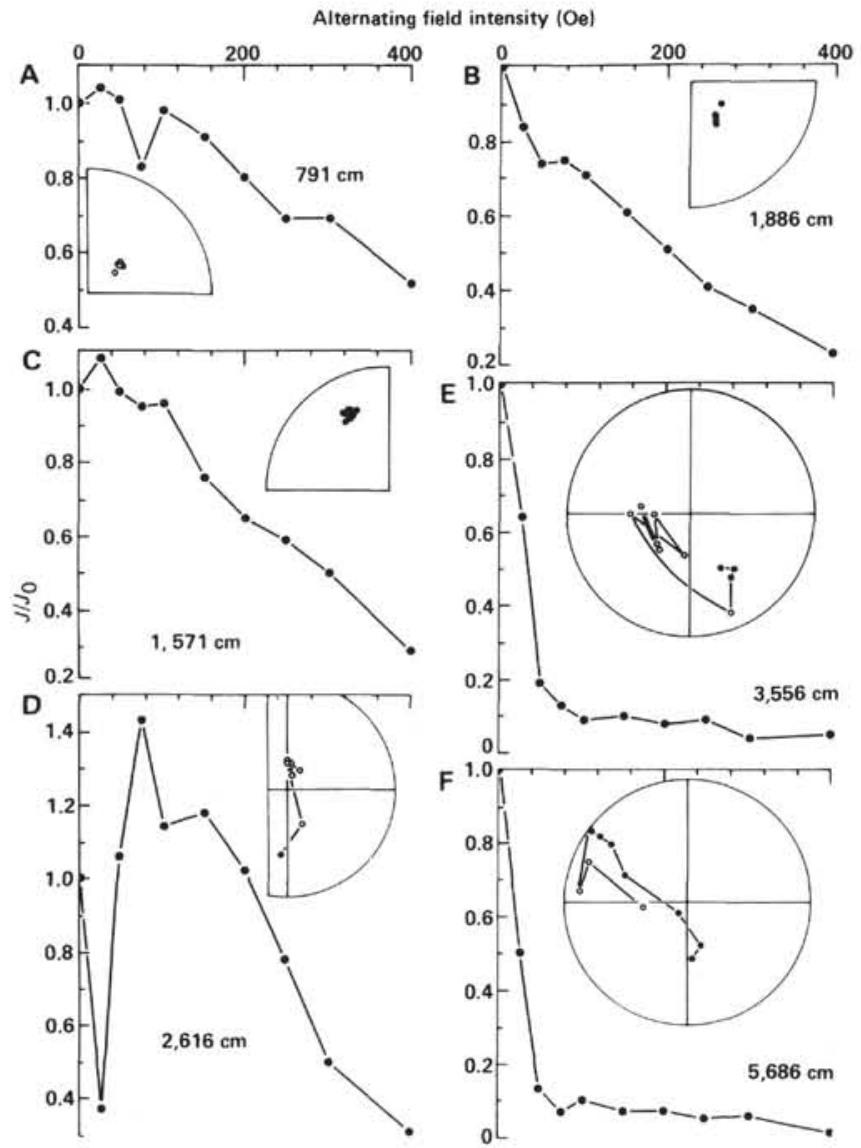

Figure 5. Demagnetization curves for typical samples from Site 576. (A) Sample 576-2-1, $96 \mathrm{~cm}$, (B) Sample 576-4-1, $106 \mathrm{~cm}$ and (C) Sample 576-2-6, $126 \mathrm{~cm}$ yield stable estimates of detrital remanence, whereas (D) Sample 576-4-6, $86 \mathrm{~cm}$, (E) Sample 576-6-3, $116 \mathrm{~cm}$, and (F) Sample 576-8-2, $126 \mathrm{~cm}$ from the dark brown manganese-rich clays do not yield reliable detrital remanence values. 
Table 6. Magnetic properties of samples from Site 578.

\begin{tabular}{|c|c|c|c|c|c|c|c|c|c|c|}
\hline 竞 & ปั & 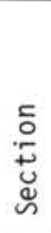 & 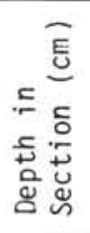 & 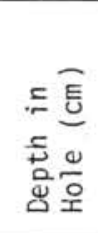 & 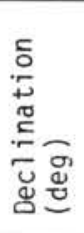 & 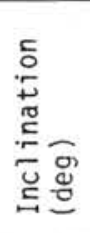 & NRP (emu) & ${ }^{3}, 00$ (emu) & ${ }^{J_{100}}{ }^{/ J_{0}}$ & Note \\
\hline 901 & 1 & 1 & 16 & 15 & 173 & 47 & $0.9823=-04$ & $0.88135-04$ & 0.897 & 11 \\
\hline 903 & 1 & 1 & 53 & 53 & 150 & 53 & $0.8811 E-04$ & $0.7592 E-04$ & 0.862 & L 1 \\
\hline 905 & 1 & 1 & 86 & 86 & 116 & 44 & $0.1419 E-04$ & $0.1039 E-04$ & 0.732 & 41 \\
\hline 906 & 1 & 1 & 106 & 106 & 96 & 52 & $0.4563 E-04$ & $0.39435-04$ & 0.864 & L 1 \\
\hline 907 & 1 & 1 & 126 & 126 & & & $0.5234 E-04$ & & & \\
\hline 908 & 1 & 1 & 146 & 146 & 91 & 55 & $0.63195-04$ & $0.5353 E-04$ & 0.847 & $1-1$ \\
\hline 909 & 1 & 2 & 6 & 156 & 97 & 55 & $0.5295 E-04$ & $0.4980 E-04$ & 0.940 & $\div 1$ \\
\hline 911 & 1 & 2 & 50 & 200 & 79 & 50 & $0.5279 E-04$ & $0.38965-04$ & 0.738 & $\llcorner 1$ \\
\hline 913 & 1 & 2 & 86 & 236 & 96 & 55 & $0.2965 \varepsilon-04$ & $0.28095-04$ & 0.948 & $\llcorner 1$ \\
\hline 915 & 1 & 2 & 126 & 276 & 112 & 64 & $0.4481 E-04$ & $0.3993 E-04$ & 0.891 & -1 \\
\hline 916 & 1 & 2 & 146 & 296 & 82 & 54 & $0.49555-04$ & $0.3969 E-04$ & 0.801 & -1 \\
\hline 917 & 1 & 3 & 6 & 306 & 117 & 48 & & $0.5108 E-04$ & & $\perp 1$ \\
\hline 918 & 1 & 3 & 27 & 327 & 136 & 47 & $0.7817 E-04$ & $0.6858 E-04$ & 0.877 & $\leq 1$ \\
\hline 919 & 1 & 3 & 46 & 346 & 126 & 51 & & $0.7448 E-04$ & & L 1 \\
\hline 920 & 1 & 3 & 66 & 366 & 130 & 30 & $0.58945-04$ & $0.4499 E-04$ & 0.763 & L 1 \\
\hline 921 & 1 & 3 & 86 & 386 & 149 & 41 & $0.5645 E-04$ & $0.4376 E-04$ & 0.775 & L 1 \\
\hline 923 & 1 & 3 & 126 & 426 & 153 & 44 & $0.6618 E-04$ & $0.55425-04$ & 0.837 & L1 \\
\hline 925 & 2 & 1 & 6 & 486 & 201 & 40 & $0.6386 E-04$ & $0.5296 E-04$ & 0.829 & L 1 \\
\hline 927 & 2 & 1 & 43 & 523 & 219 & 56 & $0.5751 E-04$ & $0.4656 E-04$ & 0.810 & 11 \\
\hline 929 & 2 & 1 & 86 & 566 & 218 & 30 & $0.3148 E-04$ & $0.2548 E-04$ & 0.809 & L 1 \\
\hline 931 & 2 & 1 & 126 & 606 & 251 & 62 & $0.5760 E-04$ & $0.5276 E-04$ & 0.916 & L 1 \\
\hline 932 & 2 & 1 & 146 & 626 & 232 & 48 & $0.5948 E-04$ & $0.5291 E-04$ & 0.890 & L 1 \\
\hline 933 & 2 & 2 & 5 & 635 & 232 & -28 & $0.3430 E-04$ & $0.26365-04$ & 0.768 & L 1 \\
\hline 934 & 2 & 2 & 26 & 656 & 237 & 42 & $0.5290 E-04$ & $0.4363 E-04$ & 0.825 & L 1 \\
\hline 935 & 2 & 2 & 46 & 676 & 222 & 54 & $0.4485 E-04$ & $0.3730 E-04$ & 0.832 & 11 \\
\hline 937 & 2 & 2 & 86 & 716 & 242 & 54 & $0.5689 E-04$ & $0.4919 E-04$ & 0.865 & L 1 \\
\hline 939 & 2 & 2 & 126 & 756 & 235 & 64 & $0.5176 E-04$ & $0.45325-04$ & 0.876 & L 1 \\
\hline 941 & 2 & 3 & 16 & 796 & 243 & 37 & $0.2839 E-04$ & $0.2359 E-04$ & 0.831 & $L 1, F$ \\
\hline 942 & 2 & 3 & 41 & 821 & 234 & 48 & $0.1158 E-03$ & $0.8305 E-04$ & 0.717 & 11 \\
\hline 943 & 2 & 3 & 66 & 846 & 189 & 19 & $0.6138 E-04$ & $0.5086 z-04$ & 0.829 & $\div 1$ \\
\hline 944 & 2 & 3 & 86 & 866 & 1.76 & 55 & $0.5493 E-04$ & $0.4996 E-04$ & 0.909 & L1 \\
\hline 945 & 2 & 3 & 106 & 886 & 178 & 51 & $0.66495-04$ & $0.5105 E-04$ & 0.768 & L 1 \\
\hline 947 & 2 & 3 & 144 & 924 & 172 & 54 & $0.45975-04$ & $0.3624 E-04$ & 0.788 & L 1 \\
\hline 949 & 2 & 4 & 26 & 956 & 167 & 59 & $0.86495-04$ & $0.7093 E-04$ & 0.820 & L 1 \\
\hline 951 & 2 & 4 & 64 & 994 & 159 & 61 & $0.80735-04$ & $0.6583 E-04$ & 0.815 & L 1 \\
\hline 953 & 2 & 4 & 106 & 1036 & 174 & 49 & $0.5319 E-04$ & $0.4363 E-04$ & 0.820 & 11 \\
\hline 955 & 2 & 4 & 146 & 1076 & 178 & 49 & $0.5833 E-05$ & $0.4645 \Xi-05$ & 0.796 & 41 \\
\hline 957 & 2 & 5 & 26 & 1106 & 177 & 59 & $0.6167 E-04$ & $0.5225 E-04$ & 0.847 & L 1 \\
\hline 959 & 2 & 5 & 66 & 1146 & 187 & 60 & $0.7992 E-04$ & $0.68155-04$ & 0.853 & L 1 \\
\hline 961 & 2 & 5 & 106 & 1186 & 180 & 59 & $0.8308 \vec{E}-04$ & $0.5693 E-04$ & 0.806 & 41 \\
\hline 963 & 2 & 6 & 6 & 1236 & 186 & 55 & $0.9513 E-04$ & $0.7451 E-04$ & 0.783 & L 1 \\
\hline 965 & 3 & 1 & 4 & 1434 & 351 & 45 & $0.1195 E-03$ & $0.9947 E-04$ & 0.832 & 11 \\
\hline 967 & 3 & 1 & 46 & 1476 & 3 & 64 & $0.8910 E-05$ & $0.7434 E-05$ & 0.834 & $\llcorner 1$ \\
\hline 969 & 3 & 1 & 86 & 1516 & 355 & 54 & $0.20575-04$ & $0.1716 E-04$ & 0.834 & L 1 \\
\hline 970 & 3 & 1 & 106 & 1536 & 5 & 53 & $0.4809 E-04$ & $0.4028 E-04$ & 0.838 & L 1 \\
\hline 971 & 3 & 1 & 126 & 1556 & 5 & -58 & $0.3841 E-04$ & $0.3398 E-04$ & 0.885 & L 1 \\
\hline 972 & 3 & 1 & 146 & 1576 & 357 & 47 & $0.4936 \varepsilon-04$ & $0.4115 E-04$ & 0.825 & $\llcorner 1$ \\
\hline 973 & 3 & 2 & 6 & 1586 & 9 & 55 & $0.4534 E-04$ & $0.3737 E-04$ & 0.824 & L 1 \\
\hline 975 & 3 & 2 & 46 & 1626 & 358 & 51 & $0.2706 E-04$ & $0.2394 E-04$ & 0.885 & -1 \\
\hline 977 & 3 & 2 & 86 & 1666 & 8 & 60 & $0.6184 E-04$ & $0.5196 E-04$ & 0.840 & L 1 \\
\hline 979 & 3 & 2 & 126 & 1706 & 18 & 58 & $0.5784 E-04$ & $0.4808 E-04$ & 0.831 & $\llcorner 1$ \\
\hline 980 & 3 & 2 & 146 & 1726 & 23 & 54 & $0.5837 E-04$ & $0.49575-04$ & 0.849 & 11 \\
\hline 981 & 3 & 3 & 6 & 1736 & 52 & -13 & $0.5258 E-04$ & $0.4256 E-04$ & 0.809 & L 1 \\
\hline 982 & 3 & 3 & 26 & 1756 & 15 & 53 & $0.8643 E-04$ & $0.7252 E-04$ & 0.839 & L 1 \\
\hline 983 & 3 & 3 & 46 & 1776 & 28 & 55 & $0.5266 E-04$ & $0.4174 E-04$ & 0.793 & L 1 \\
\hline 985 & 3 & 3 & 83 & 1813 & 22 & 56 & $0.5009 E-04$ & $0.5335 E-04$ & 0.888 & L 1 \\
\hline 987 & 3 & 3 & 126 & 1856 & 38 & 54 & $0.7511 E-04$ & $0.6474 E-04$ & 0.862 & $L 1$ \\
\hline 989 & 3 & 4 & 6 & 1886 & 34 & 52 & $0.6767 E-04$ & $0.5842 E-04$ & 0.863 & L 1 \\
\hline 991 & 3 & 4 & 46 & 1926 & 44 & 54 & $0.5153 E-04$ & $0.4372 E-04$ & 0.848 & 41 \\
\hline 993 & 3 & 4 & 86 & 1966 & 43 & 54 & $0.5618 E-04$ & $0.5377 E-04$ & 0.957 & $L 1$ \\
\hline
\end{tabular}


Table 6. (Continued).

\begin{tabular}{|c|c|c|c|c|c|c|c|c|c|c|}
\hline $\begin{array}{l}\frac{\Delta}{a} \\
\text { 怘 }\end{array}$ & ปั & 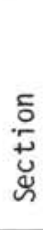 & 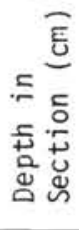 & 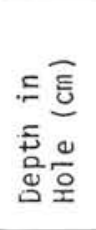 & 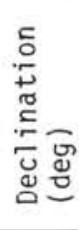 & 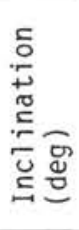 & NRM (erinu) & ${ }^{J} 100$ (emu) & ${ }^{3} 100^{1}{ }_{0}$ & Note \\
\hline 995 & 3 & 4 & 126 & 2006 & 49 & 57 & $0.7995 E-04$ & $0.7084 E-04$ & 0.886 & L 1 \\
\hline 997 & 3 & 5 & 6 & 2036 & 50 & 48 & $0.9282 E-04$ & $0.8056 E-04$ & 0.868 & \pm 1 \\
\hline 999 & 3 & 5 & 46 & 2076 & 48 & 54 & $0.7261 \mathrm{E}-04$ & $0.6503 E-04$ & 0.896 & L 1 \\
\hline 1001 & 3 & 5 & 86 & 2116 & 58 & 58 & $0.7631 E-04$ & $0.6187 E-04$ & 0.811 & L 1 \\
\hline 1003 & 3 & 5 & 126 & 2156 & 67 & 52 & $0.1058 E-03$ & $0.93295-04$ & 0.882 & L 1 \\
\hline 1005 & 3 & 6 & 6 & 2186 & 79 & 48 & $0.4716 E-04$ & $0.38935-04$ & 0.825 & -1 \\
\hline 1007 & 3 & 6 & 50 & 2230 & 79 & 66 & $0.8473 E-05$ & $0.6610 E-05$ & 0.780 & ᄂ 1 \\
\hline 1009 & 3 & 6 & 86 & 2266 & 63 & 56 & $0.2053 E-05$ & $0.1705 E-05$ & 0.830 & $\llcorner 1$ \\
\hline 1011 & 3 & 7 & 6 & 2336 & 67 & 49 & $0.4198 E-04$ & $0.3244 E-04$ & 0.773 & L 1 \\
\hline 1013 & 3 & 7 & 46 & 2376 & 66 & 53 & $0.3954 E-04$ & $0.3326 E-04$ & 0.841 & $1-1$ \\
\hline 1015 & 4 & 1 & 26 & 2405 & 333 & 75 & $0.9327 E-05$ & $0.7909 E-05$ & 0.848 & 11 \\
\hline 1017 & 4 & 1 & 66 & 2446 & 329 & 47 & $0.4799 E-04$ & $0.4128 E-04$ & 0.860 & L 1 \\
\hline 1019 & 4 & 1 & 113 & 2493 & 340 & 47 & $0.4362 E-04$ & $0.3854 E-04$ & 0.883 & $\llcorner 1$ \\
\hline 1021 & 4 & 1 & 145 & 2525 & 329 & 53 & $0.4006 E-04$ & $0.3469 E-04$ & 0.866 & L 1 \\
\hline 1023 & 4 & 2 & 25 & 2555 & 328 & 46 & $0.4658 E-04$ & $0.3951 E-04$ & 0.848 & L 1 \\
\hline 1025 & 4 & 2 & 66 & 2596 & 321 & 51 & $0.6295 E-04$ & $0.5487 E-04$ & 0.872 & L 1 \\
\hline 1027 & 4 & 2 & 108 & 2638 & 329 & 58 & $0.6493 E-04$ & $0.5450 E-04$ & 0.839 & L 1 \\
\hline 1029 & 4 & 2 & 146 & 2676 & 332 & 56 & $0.5844 \Xi-04$ & $0.5307 E-04$ & 0.908 & $\measuredangle 1$ \\
\hline 1039 & 4 & 3 & 26 & 2706 & 312 & 50 & $0.4358 E-04$ & $0.3634 E-04$ & 0.834 & $\llcorner 1$ \\
\hline 1041 & 4 & 3 & 66 & 2746 & 314 & 29 & $0.7841 E-04$ & $0.6466 \mathrm{E}-04$ & 0.825 & $1-1$ \\
\hline 1042 & 4 & 3 & 86 & 2766 & 266 & 49 & $0.4281 E-04$ & $0.3593 E-04$ & 0.839 & $L 1$ \\
\hline 1043 & 4 & 3 & 105 & 2785 & 116 & -12 & $0.6468 E-05$ & $0.8880 E-05$ & 1.373 & A $2-3$ \\
\hline 1031 & 4 & 4 & 25 & 2855 & 99 & -58 & $0.4112 E-04$ & $0.4355 E-04$ & 1.059 & LI \\
\hline 1033 & 4 & 4 & 65 & 2895 & 65 & -29 & $9 E-06$ & $0.6415 E-06$ & 1.243 & L 1 \\
\hline 1035 & 4 & 4 & 110 & 29 & 65 & -50 & $491 E-06$ & $0.9051 E-06$ & 0.954 & A 3-4 \\
\hline 1037 & 4 & 4 & 146 & 2976 & 68 & -52 & $0.6026 E-05$ & $5643 \leq-05$ & 0.937 & 11 \\
\hline 1044 & 4 & 3 & 126 & 2806 & 95 & -61 & $0.3614 E-04$ & $0.3452 E-04$ & 0.955 & -1 \\
\hline 1045 & 4 & 3 & 146 & 2826 & 79 & -52 & $0.5822 E-04$ & $0.6056 E-04$ & 1.040 & L 1 \\
\hline 1047 & 4 & 5 & 32 & 3012 & 50 & -50 & $0.2102 E-04$ & $0.2156 E-04$ & 1.026 & L 1 \\
\hline 1049 & 4 & 5 & 66 & 3046 & 49 & -46 & $0.9623 E-05$ & $0.9706 E-05$ & 1.009 & L 1 \\
\hline 1051 & 4 & 5 & 106 & 3086 & 41 & -59 & $0.1535 E-04$ & $0.1903 E-04$ & 1.240 & L 1 \\
\hline 1053 & 4 & 5 & 146 & 3126 & 22 & -54 & $0.4646 E-04$ & $0.5007 E-04$ & 1.078 & L 1 \\
\hline 1055 & 4 & 6 & 26 & 3156 & 10 & -55 & $0.3728 E-04$ & $0.4142 \mathrm{E}-04$ & 1.111 & L 1 \\
\hline 1056 & 4 & 6 & 46 & 3176 & 10 & -51 & $0.3980 E-04$ & $0.4380 E-04$ & 1.101 & -1 \\
\hline 1057 & 4 & 6 & 66 & 3196 & 2 & 57 & $0.2730 E-04$ & $0.3323 E-04$ & 1.217 & L 1 \\
\hline 1059 & 4 & 6 & 114 & 3244 & 161 & 56 & $0.5555 E-04$ & $0.4381 E-04$ & 0.789 & L 1 \\
\hline 1061 & 5 & 1 & 47 & 3377 & 330 & 48 & $0.5012 E-04$ & $0.4458 E-04$ & 0.889 & L 1 \\
\hline 1062 & 5 & 1 & 106 & 3436 & 2 & 67 & $0.1931 E-04$ & $0.1509 E-04$ & 0.781 & L 1 \\
\hline 1063 & 5 & 1 & 126 & 3456 & 154 & -57 & $0.3798 E-04$ & $0.3878 E-04$ & 1.021 & L 1 \\
\hline 1065 & 5 & 2 & 6 & 3486 & 162 & -47 & $0.6264 E-04$ & $0.5965 E-04$ & 0.952 & 11 \\
\hline 1067 & 5 & 2 & 51 & 3531 & 156 & -42 & $0.4311 E-04$ & $0.4515 E-04$ & 1.047 & 11 \\
\hline 1069 & 5 & 2 & 85 & 3565 & 143 & -39 & $0.1035 E-04$ & $0.1277 E-04$ & 1.234 & L 1 \\
\hline 1071 & 5 & 2 & 126 & 3606 & 167 & -49 & $0.5329 E-04$ & $0.5386 E-04$ & 1.011 & L 1 \\
\hline 1073 & 5 & 3 & 6 & 3636 & 165 & -49 & $0.6879 E-04$ & $0.6906 E-04$ & 1.004 & L 1 \\
\hline 1075 & 5 & 3 & 45 & 3675 & 168 & -51 & $0.4353 E-04$ & $0.4475 E-04$ & 1.028 & L 1 \\
\hline 1077 & 5 & 3 & 86 & 3716 & 167 & -52 & $0.3510 E-04$ & $0.3907 E-04$ & 1.113 & L 1 \\
\hline 1078 & 5 & 3 & 105 & 3735 & 178 & -44 & $0.1619 E-04$ & $0.1990 \mathrm{E}-04$ & 1.230 & L 1 \\
\hline 1079 & 5 & 3 & 128 & 3758 & 356 & 44 & $0.2183 E-04$ & $0.1661 E-04$ & 0.761 & L 1 \\
\hline 1080 & 5 & 3 & 146 & 3776 & 46 & -45 & $0.4044 E-04$ & $0.4543 E-05$ & 0.112 & 11 \\
\hline 1081 & 5 & 4 & 6 & 3786 & 167 & -38 & $0.1102 E-04$ & $0.1270 E-04$ & 1.153 & L 1 \\
\hline 1083 & 5 & 4 & 46 & 3826 & 176 & -46 & $0.3321 E-04$ & $0.3177 E-04$ & 0.957 & L 1 \\
\hline 1085 & 5 & 4 & 86 & 3866 & 174 & -48 & $0.3601 \mathrm{E}$ & $3666 E-04$ & 1.018 & L 1 \\
\hline 1087 & 5 & 4 & 126 & 3906 & 181 & -52 & $0.1873 E-04$ & $0.1999 E-04$ & 1.067 & L 1 \\
\hline 1089 & 5 & 5 & 6 & 3936 & 151 & -52 & $0.6993 E-06$ & $0.5485 E-06$ & 0.784 & L 1 \\
\hline 1091 & 5 & 5 & 46 & 3976 & 184 & -54 & $0.9561 E-05$ & $0.8065 E-05$ & 0.844 & L 1 \\
\hline 1093 & 5 & 5 & 86 & 4015 & 197 & -52 & $0.2632 E-04$ & $0.2318 E-04$ & 0.881 & L 1 \\
\hline 1095 & 5 & 5 & 128 & 4058 & 175 & -51 & $0.1753 E-04$ & $0.1544 E-04$ & 0.881 & 1.1 \\
\hline 1097 & 5 & 6 & 6 & 4086 & 188 & -52 & $0.2485 E-04$ & $0.2129 E-04$ & 0.857 & L 1 \\
\hline 1099 & 5 & 6 & 46 & 4126 & 177 & -52 & $0.6102 \mathrm{E}-04$ & $0.5938 E-04$ & 0.973 & L 1 \\
\hline 1101 & 5 & 6 & 86 & 4166 & 191 & -54 & $0.7963 E-04$ & $0.7240 E-04$ & 0.909 & L 1 \\
\hline
\end{tabular}


Table 6. (Continued).

\begin{tabular}{|c|c|c|c|c|c|c|c|c|c|c|}
\hline 吕 & ¿ั่ & $\begin{array}{l}\text { 듬 } \\
+\frac{1}{4} \\
\text { ज }\end{array}$ & 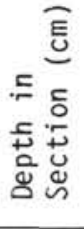 & 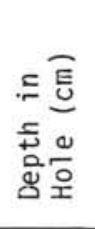 & 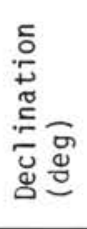 & 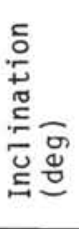 & NRR (emu) & ${ }^{\mathrm{J}} 100$ (emu) & ${ }_{100} / J_{0}$ & Note \\
\hline 1103 & 5 & 6 & 126 & 4206 & 194 & -55 & $0.7699 E-04$ & $0.7254 \mathrm{E}-04$ & 0.942 & -1 \\
\hline 1105 & 5 & 7 & 22 & 4252 & 190 & -56 & $0.4073 E-04$ & $0.3743 E-04$ & 0.919 & ᄂ 1 \\
\hline 1107 & 6 & 1 & 6 & 4286 & & & $0.45705-05$ & $0.2415 E-05$ & 0.529 & $F$ \\
\hline 1109 & 6 & 1 & 67 & 4347 & 31 & -51 & $0.3309 E-04$ & $0.3485 E-04$ & 1.053 & $\llcorner 1$ \\
\hline 1111 & 6 & 1 & 106 & 4386 & 43 & -57 & $0.4049 E-04$ & $0.35985-04$ & 0.889 & $\llcorner 1$ \\
\hline 1113 & 6 & 1 & 146 & 4426 & 63 & -56 & $0.1237 \vec{z}-04$ & $0.1056 E-04$ & 0.854 & $\llcorner 1$ \\
\hline 1115 & 6 & 2 & 26 & 4456 & 35 & -48 & $0.3326 E-04$ & $0.3226 F-04$ & 0.970 & L 1 \\
\hline 1117 & 6 & 2 & 66 & 4496 & 53 & -51 & $0.3450 E-04$ & $0.3643 E-04$ & 1.056 & $\llcorner 1$ \\
\hline 1119 & 6 & 2 & 106 & 4536 & 56 & -41 & $0.5831 \overrightarrow{\mathrm{e}}-04$ & $0.5671 \mathrm{E}-04$ & 0.972 & LI \\
\hline 1121 & 6 & 2 & 146 & 4576 & 71 & -46 & $0.7053 E-04$ & $0.7330 E-04$ & 1.039 & L 1 \\
\hline 1123 & 6 & 3 & 26 & 4606 & 66 & -55 & $0.50795-04$ & $0.4799 E-04$ & 0.945 & -1 \\
\hline 1125 & 6 & 3 & 63 & 4643 & 81 & -54 & $0.7311 E-04$ & $0.7222 E-04$ & 0.988 & L 1 \\
\hline 1127 & 6 & 3 & 102 & 4682 & 88 & -53 & $45-04$ & $4 E-04$ & 0.976 & L 1 \\
\hline 1129 & 6 & 3 & 146 & 4726 & 63 & -57 & $0.6839 E-06$ & 0.69 & 1.012 & ᄂ 1 \\
\hline 1131 & 6 & 4 & 24 & 4756 & 81 & -48 & $0.2404 E-05$ & $0.2218 E-05$ & 0.923 & L 1 \\
\hline 1133 & 6 & 4 & 66 & 4796 & 86 & -52 & $0.1841 E-04$ & $0.16995-04$ & 0.923 & -1 \\
\hline 1135 & 6 & 4 & 106 & 4836 & 83 & -51 & $0.2761 E-04$ & $0.2668 E-04$ & 0.966 & ᄂ 1 \\
\hline 1137 & 6 & 4 & 146 & 4876 & 99 & -55 & $0.2893 \Xi-04$ & $0.3367 E-04$ & 1.164 & $1-1$ \\
\hline 1139 & 6 & 5 & 26 & 4906 & 90 & -55 & $0.6737 E-04$ & $0.6803 E-04$ & 1.010 & $\llcorner 1$ \\
\hline 1141 & 6 & 5 & 66 & 4946 & 68 & -59 & $0.3964 E-04$ & $0.4335 E-04$ & 1.094 & $\llcorner 1$ \\
\hline 1143 & 6 & 5 & 106 & 4986 & 67 & -52 & $0.2143 E-04$ & $0.2457 E-04$ & 1.146 & $\longrightarrow 1$ \\
\hline 1145 & 6 & 5 & 146 & 5026 & 79 & -49 & $0.2629 E-04$ & $0.2353 E-04$ & 0.895 & 1 \\
\hline 1147 & 6 & 6 & 34 & 5064 & 72 & -37 & $0.1374 E-05$ & $0.1244 E-05$ & 0.906 & L 1 \\
\hline 1149 & 6 & 6 & 86 & 5116 & 75 & -50 & $0.2321 E-04$ & $0.2111 E-04$ & 0.910 & L 1 \\
\hline 1151 & 6 & 7 & 3 & 5183 & 73 & -56 & $0.4278 E-04$ & $0.4016 E-04$ & 0.939 & 11 \\
\hline 1153 & 6 & 7 & 43 & 5223 & 70 & -57 & $0.2653 E-04$ & $0.2431 \mathrm{E}-04$ & 0.916 & ᄂ 1 \\
\hline 1155 & 7 & 1 & 46 & 5266 & 301 & -38 & $0.3578 E-04$ & $0.3165 E-04$ & 0.884 & L 1 \\
\hline 1157 & 7 & 1 & 86 & 5306 & 298 & -60 & $0.72815-04$ & $0.6341 \mathrm{E}-04$ & 0.871 & 1.1 \\
\hline 1159 & 7 & 1 & 126 & 5346 & 298 & -61 & $0.1145 E-04$ & $0.1157 E-04$ & 1.011 & L 1 \\
\hline 1160 & 7 & 1 & 146 & 5366 & 122 & 50 & $0.3663 E-04$ & $0.3182 E-04$ & 0.869 & $\llcorner 1$ \\
\hline 1161 & 7 & 2 & 6 & 5376 & 123 & 58 & $0.3285 E-04$ & $0.2974 E-04$ & 0.905 & L 1 \\
\hline 1162 & 7 & 2 & 28 & 5398 & 263 & -44 & $0.1595 \subseteq-04$ & $0.1418 E-04$ & 0.889 & 41 \\
\hline 1163 & 7 & 2 & 46 & 5416 & 114 & 47 & $0.3948 E-04$ & $0.3696 E-04$ & 0.936 & 41 \\
\hline 1165 & 7 & 2 & 86 & 5456 & 133 & 47 & $0.6829 E-04$ & $0.5882 E-04$ & 0.861 & LI \\
\hline 1167 & 7 & 2 & 131 & 5501 & 122 & 49 & $0.5317 E-04$ & $0.4489 E-04$ & 0.844 & $\llcorner 1$ \\
\hline 1169 & 7 & 3 & 6 & 5526 & 116 & 46 & $0.4255 \Xi-04$ & $0.3519 E-04$ & 0.827 & L. 1 \\
\hline 1171 & 7 & 3 & 46 & 5566 & 129 & 46 & $0.4265 E-04$ & $0.3646 \mathrm{E}-04$ & 0.855 & $\llcorner 1$ \\
\hline 1173 & 7 & 3 & 86 & 5606 & 122 & 58 & $0.6792 E-04$ & $8 \Xi-04$ & 1.026 & -1 \\
\hline 1175 & 7 & 3 & 126 & 5646 & 125 & 58 & $0.6372 E-04$ & -04 & 0.836 & 11 \\
\hline 1177 & 7 & 4 & 6 & 5676 & 143 & 57 & $0.8232 E-04$ & $7665 E-04$ & 0.931 & L 1 \\
\hline 1179 & 7 & 4 & 26 & 5717 & 140 & 52 & $0.5154 \mathrm{E}-04$ & $0.4050 E-04$ & 0.786 & L 1 \\
\hline 1181 & 7 & 4 & 86 & 5756 & 148 & 58 & $0.8095 E-04$ & $0.7638 E-04$ & 0.944 & -1 \\
\hline 1183 & 7 & 4 & 126 & 5796 & 160 & 50 & $0.2698 E-04$ & $0.2158 E-04$ & 0.800 & L 1 \\
\hline 1184 & 7 & 4 & 146 & 5816 & 325 & -56 & $0.2340 E-04$ & $0.2715 E-04$ & 1.160 & L 1 \\
\hline 1185 & 7 & 5 & 6 & 5826 & 328 & -55 & $0.3487 E-04$ & $0.3819 E-04$ & 1.095 & 41 \\
\hline 1187 & 7 & 5 & 46 & 5866 & 316 & -78 & $0.1187 E-04$ & $0.1923 E-04$ & 1.619 & L 1 \\
\hline 1189 & 7 & 5 & 66 & 5906 & 321 & -49 & $0.6215 E-04$ & $0.6744 E-04$ & 1.085 & L 1 \\
\hline 1191 & 7 & 5 & 126 & 5946 & 312 & -56 & $0.6257 E-04$ & $0.6426 E-04$ & 1.027 & L 1 \\
\hline 1193 & 7 & 6 & 6 & 5976 & 306 & -46 & $0.4563 E-04$ & $0.4633 E-04$ & 1.015 & L 1 \\
\hline 1195 & 7 & 6 & 45 & 6015 & 315 & -48 & $0.5123 E-04$ & $0.5166 E-04$ & 1.008 & L 1 \\
\hline 1197 & 7 & 6 & 86 & 6056 & 323 & -47 & $0.4669 E-04$ & $0.5263 E-04$ & 1.127 & L 1 \\
\hline 1199 & 7 & 6 & 126 & 6096 & 308 & -50 & $0.4198 E-04$ & $0.4282 E-04$ & 1.020 & L 1 \\
\hline 1201 & 7 & 7 & 11 & 6131 & 304 & -58 & $0.5380 E-04$ & $0.5343 E-04$ & 0.993 & -1 \\
\hline 1202 & 7 & 7 & 46 & 6166 & 295 & -59 & $0.4657 E-04$ & $0.4697 E-04$ & 1.009 & L 1 \\
\hline 1203 & 8 & 1 & 26 & 6206 & 165 & 54 & $0.1504 E-04$ & $0.1333 E-04$ & 0.886 & L 1 \\
\hline 1204 & 8 & 1 & 46 & 6226 & 315 & -62 & $0.1115 E-05$ & $0.1064 E-05$ & 0.954 & $\llcorner 1$ \\
\hline 1205 & 8 & 1 & 66 & 6246 & 272 & 42 & $0.1202 E-04$ & $0.1035 E-04$ & 0.861 & 1.1 \\
\hline 1206 & 8 & 1 & 87 & 6267 & 75 & -59 & $0.74725-06$ & $0.6568 E-06$ & 0.879 & 41 \\
\hline 1207 & 8 & 1 & 92 & 6272 & 92 & -50 & $0.1924 \varepsilon-05$ & $0.1868 E-05$ & 0.971 & L 1 \\
\hline 1209 & 8 & 1 & 126 & 6306 & 81 & -53 & $0.1483 E-05$ & $0.1404 E-05$ & 0.947 & L 1 \\
\hline
\end{tabular}


Table 6. (Continued).

\begin{tabular}{|c|c|c|c|c|c|c|c|c|c|c|}
\hline$\frac{\frac{0}{0}}{\stackrel{5}{心}}$ & ১े & 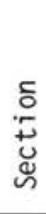 & 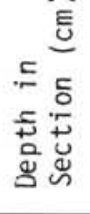 & $\begin{array}{l}\subseteq \text { 틉 } \\
\text { 동 } \\
\text { 응웅 }\end{array}$ & 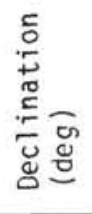 & 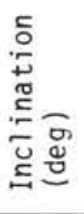 & NRM (emu) & ${ }^{J} 100(\mathrm{emu})$ & ${ }^{3} 100^{/ 3} 0$ & Note \\
\hline 1211 & 8 & 2 & 6 & 6336 & 73 & -45 & $0.2489 E-05$ & $0.2406 E-05$ & 0.967 & L 1 \\
\hline 1213 & 8 & 2 & 46 & 6376 & 61 & -51 & $0.4387 E-04$ & $0.39525-04$ & 0.901 & L 1 \\
\hline 1215 & 8 & 2 & 86 & 6415 & 64 & -49 & $0.6411 \mathrm{E}-04$ & $0.5885 E-04$ & 0.918 & L 1 \\
\hline 1217 & 8 & 2 & 126 & 6456 & 54 & -55 & $0.6024 \sqsubseteq-04$ & $0.5416 E-04$ & 0.899 & $\llcorner 1$ \\
\hline 1219 & 8 & 3 & $\sigma$ & 6486 & 68 & -56 & $0.6618 E-04$ & $0.6401 E-04$ & 0.967 & $\llcorner 1$ \\
\hline 1221 & 8 & 3 & 46 & 6526 & 64 & -56 & $0.7144 \mathrm{E}-04$ & $0.6561 E-04$ & 0.918 & L 1 \\
\hline 1223 & 8 & 3 & 86 & 6566 & 65 & -50 & $0.5553 E-04$ & $0.5890 E-04$ & 1.061 & L 1 \\
\hline 1225 & 8 & 3 & 126 & 6606 & 65 & -46 & $0.4465 E-04$ & $0.4530 E-04$ & 1.014 & -1 \\
\hline 1227 & 8 & 4 & 6 & 6636 & 48 & -43 & $0.4288 E-04$ & $0.4636 E-04$ & 1.081 & $\llcorner 1$ \\
\hline 1229 & 8 & 4 & 48 & 6678 & 52 & -51 & $0.6347 E-04$ & $0.6089 E-04$ & 959 & L 1 \\
\hline 1231 & 8 & 4 & 86 & 6716 & 59 & -34 & $0.4446 E-04$ & $0.4295 E-04$ & 0.966 & $\llcorner 1$ \\
\hline 1233 & 8 & 4 & 126 & 6756 & 64 & -44 & $0.4565 \Xi-04$ & $0.45795-04$ & 1.003 & 41 \\
\hline 1235 & 8 & 5 & 6 & 6786 & 61 & -41 & $0.4855 E-04$ & $0.4737 E-04$ & 0.976 & L 1 \\
\hline 1237 & 8 & 5 & 46 & 6826 & 70 & -43 & $0.4080 E-04$ & $0.40015-04$ & 0.981 & L 1 \\
\hline 1239 & 8 & 5 & 86 & 6866 & 73 & -45 & $0.1511 E-04$ & $0.1806 E-04$ & 1.195 & $\llcorner 1$ \\
\hline 1241 & 8 & 5 & 123 & 6903 & 78 & -50 & $0.5791 \mathrm{E}-04$ & $0.6302 E-04$ & 1.088 & $\llcorner 1$ \\
\hline 1243 & 8 & 6 & 6 & 6936 & 82 & -49 & $0.3933 E-04$ & $0.4270 E-04$ & 1.086 & L 1 \\
\hline 1245 & 8 & 6 & 46 & 6976 & 73 & -56 & $0.1046 E-03$ & $0.1023 E-03$ & 0.977 & L 1 \\
\hline 1247 & 8 & 6 & 91 & 7021 & 83 & -60 & $0.7549 E-04$ & $0.7968 E-04$ & 1.056 & $\leftarrow 1$ \\
\hline 1249 & 8 & 6 & 126 & 7056 & 75 & -47 & $0.6629 E-04$ & $0.6296 E-04$ & 0.950 & L 1 \\
\hline 1251 & 8 & 7 & 5 & 7085 & 75 & -49 & $0.8053 E-04$ & $0.7936 E-04$ & 0.985 & L 1 \\
\hline 1253 & 8 & 7 & 36 & 7116 & 88 & -55 & $0.8623 E-04$ & $0.9006 \mathrm{E}-04$ & 1.044 & $\downarrow 1$ \\
\hline 1255 & 9 & 1 & 31 & 7161 & 77 & -43 & $0.16025-04$ & $0.1723 E-04$ & 1.076 & L 1 \\
\hline 1257 & 9 & 1 & 66 & 7196 & 61 & -56 & $0.4017 E-04$ & $0.4508 E-04$ & 1.122 & -1 \\
\hline 1258 & 9 & 1 & 86 & 7216 & 67 & 32 & $0.5689 E-04$ & $0.6237 E-04$ & 1.096 & $\llcorner 1$ \\
\hline 1259 & 9 & 1 & 106 & 7236 & 71 & -42 & $0.6136 \mathrm{E}-04$ & $0.6231 \mathrm{E}-04$ & 1.015 & -1 \\
\hline 1260 & 9 & 1 & 126 & 7256 & 58 & -45 & $0.1512 \mathrm{E}-0.4$ & $0.1930 E-04$ & 1.276 & L 1 \\
\hline 1261 & 9 & 2 & 146 & 7276 & 249 & 50 & $0.4425 E-04$ & $0.3795 E-04$ & 0.858 & -1 \\
\hline 1263 & 9 & 2 & 26 & 7306 & 61 & 48 & $0.7538 \mathrm{E}-04$ & $0.7155 E-04$ & 0.949 & L 1 \\
\hline 1265 & 9 & 2 & 67 & 7347 & 49 & 55 & $0.5244 E-04$ & $0.5327 E-04$ & 0.853 & L 1 \\
\hline 1267 & 9 & 2 & 106 & 7386 & 78 & 61 & $0.3821 E-04$ & $0.3532 E-04$ & 0.924 & LI \\
\hline 1269 & 9 & 2 & 146 & 7426 & 61 & 49 & $0.1010 E-03$ & $0.8029 E-04$ & 0.795 & 1.1 \\
\hline 1271 & 9 & 3 & 26 & 7456 & 112 & 42 & $0.86635-04$ & $0.8172 E-04$ & 0.943 & $\checkmark 1$ \\
\hline 1273 & 9 & 3 & 65 & 7496 & 99 & 49 & $0.4996 E-04$ & $0.4038 E-04$ & 0.808 & L 1 \\
\hline 1275 & 9 & 3 & 106 & 7536 & 104 & 37 & $0.6787 E-04$ & $0.6201 \mathrm{E}-04$ & 0.914 & $L 1$ \\
\hline 1277 & 9 & 3 & 146 & 7576 & 117 & 45 & $0.1579 E-04$ & $0.1211 \mathrm{E}-04$ & 0.767 & $\llcorner 1$ \\
\hline 1279 & 9 & 4 & 26 & 7606 & 297 & 50 & $0.1086 E-03$ & $0.1057 E-03$ & 974 & L 1 \\
\hline 1281 & 9 & 4 & 66 & 7646 & 312 & 41 & $0.7633 E-04$ & $0.6769 \mathrm{E}-$ & 87 & -1 \\
\hline 1283 & 9 & 4 & 106 & 7686 & 313 & 48 & $0.69435-04$ & $0.5788 \varepsilon-04$ & 0.834 & 41 \\
\hline 1285 & 9 & 4 & 146 & 7726 & 314 & 50 & $0.5620 \mathrm{E}-04$ & $0.4888 E-04$ & 0.870 & L! \\
\hline 1287 & 9 & 5 & 25 & 7755 & 327 & 53 & $0.1161 E-03$ & $0.1040 E-03$ & 0.895 & L 1 \\
\hline 1289 & 9 & 5 & 66 & 7796 & 322 & 57 & $0.7050 E-04$ & $0.5330 E-04$ & 0.756 & L 1 \\
\hline 1291 & 9 & 5 & 100 & 7830 & 334 & 51 & $0.8589 E-04$ & $0.7122 E-04$ & 0.829 & L 1 \\
\hline 1293 & 9 & 6 & 6 & 7886 & 316 & 47 & $0.6791 E-04$ & $0.6092 E-04$ & 0.897 & $\llcorner 1$ \\
\hline 1295 & 9 & 6 & 46 & 7926 & 315 & 54 & $0.5598 E-04$ & $0.5325 E-04$ & 0.951 & -1 \\
\hline 1297 & 9 & 6 & 86 & 7966 & 325 & 54 & $0.5560 E-04$ & $0.4729 E-04$ & 0.851 & LI \\
\hline 1299 & 9 & 6 & 126 & 8006 & 340 & 48 & $0.8341 E-04$ & $0.7401 E-04$ & 0.887 & $\llcorner 1$ \\
\hline 1301 & 9 & 7 & 6 & 8036 & 336 & 50 & $0.8154 E-04$ & $0.7387 E-04$ & 0.906 & L 1 \\
\hline 1302 & 9 & 7 & 26 & 8056 & 305 & 59 & $0.1581 E-04$ & $0.1061 E-04$ & 0.671 & L 1 \\
\hline 1303 & 9 & 7 & 48 & 8078 & 141 & -42 & $0.1182 E-04$ & $0.1174 \mathrm{E}-04$ & 0.993 & L 1 \\
\hline 1304 & 10 & 1 & 28 & 8108 & 31 & 55 & $0.4943 E-04$ & $0.4307 E-04$ & 0.871 & $\llcorner 1$ \\
\hline 1305 & 10 & 1 & 46 & 8126 & 34 & 38 & $0.3978 \mathrm{E}-04$ & $0.3666 E-04$ & 0.922 & 1 \\
\hline 1306 & 10 & 1 & 78 & 8158 & 235 & -46 & $0.6801 \mathrm{E}-04$ & $0.7009 E-04$ & 1.031 & $\llcorner 1$ \\
\hline 1307 & 10 & 1 & 96 & 8176 & 221 & -37 & $0.2774 \mathrm{E}-04$ & $0.3139 E-04$ & 1.131 & L 1 \\
\hline 1309 & 10 & 1 & 130 & 8210 & 223 & -45 & $0.5719 \mathrm{E}-04$ & $0.6044 E-04$ & 1.057 & L 1 \\
\hline 1310 & 10 & 1 & 146 & 8226 & 217 & -49 & $0.5144 E-04$ & $0.5110 E-04$ & 0.993 & L 1 \\
\hline 1311 & 10 & 2 & 6 & 8236 & 196 & -43 & $0.3538 E-04$ & $0.3561 \mathrm{E}-04$ & 1.007 & L 1 \\
\hline 1312 & 10 & 2 & 24 & 8254 & 11 & 48 & $0.4338 E-04$ & $0.3761 \mathrm{E}-04$ & 0.867 & L 1 \\
\hline 1313 & 10 & 2 & 46 & 8276 & 11 & 45 & $0.8454 E-04$ & $0.6420 E-04$ & 0.759 & L 1 \\
\hline 1315 & 10 & 2 & 86 & 8316 & 8 & 52 & $0.7406 E-04$ & $0.6796 E-04$ & 0.918 & L 1 \\
\hline
\end{tabular}


Table 6. (Continued).

\begin{tabular}{|c|c|c|c|c|c|c|c|c|c|c|}
\hline 吕 & ذँّ & 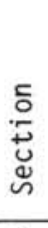 & 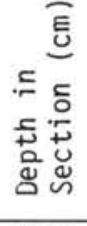 & 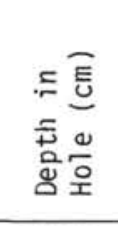 & 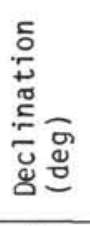 & 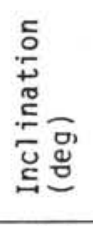 & NRM (emu) & ${ }^{J_{100}}(\mathrm{emu})$ & ${ }^{3}{ }_{100} /{ }^{3} 0$ & Note \\
\hline 1317 & 10 & 2 & 126 & 8356 & 19 & 50 & $0.4981 E-04$ & $0.4010 E-04$ & 0.805 & $\llcorner 1$ \\
\hline 1318 & 10 & 2 & 146 & 8376 & 183 & -53 & $0.1745 E-04$ & $0.26625-04$ & 1.525 & L 1 \\
\hline 1319 & 10 & 3 & 6 & 8386 & 180 & -45 & $0.4050 E-04$ & $0.4079 E-04$ & 1.007 & $\leftarrow 1$ \\
\hline 1321 & 10 & 3 & 46 & 8426 & 176 & -38 & $0.3214 E-04$ & $0.3143 E-04$ & 0.978 & L1 \\
\hline 1323 & 10 & 3 & 86 & 8466 & 178 & -42 & $0.7631 E-04$ & $0.6928 E-04$ & 0.908 & L1 \\
\hline 1324 & 10 & 3 & 106 & 8486 & 170 & -47 & $0.1547 E-04$ & $0.1733 E-04$ & 1.121 & LI \\
\hline 1325 & 10 & 3 & 126 & 8506 & 354 & 54 & $0.6684 E-04$ & $0.60425-04$ & 0.904 & $\llcorner 1$ \\
\hline 1327 & 10 & 4 & 6 & 8536 & 299 & 45 & $0.4472 E-04$ & $0.3356 E-04$ & 0.750 & L \\
\hline 1329 & 10 & 4 & 46 & 8576 & 311 & 48 & $0.6309 E-04$ & $0.5494 E-04$ & 0.871 & 11 \\
\hline 1331 & 10 & 4 & 86 & 8616 & 318 & 50 & $0.6750 E-04$ & $0.5654 E-04$ & 0.838 & L \\
\hline 1333 & 10 & 4 & 126 & 8656 & 307 & 46 & $0.60165-04$ & $0.4713 E-04$ & 0.783 & L 1 \\
\hline 1335 & 10 & 5 & 6 & 8686 & 297 & 44 & $0.6861 E-04$ & $0.5820 E-04$ & 0.848 & L 1 \\
\hline 1337 & 10 & 5 & 46 & 8726 & 294 & 46 & $E-04$ & $0.5166 E-04$ & 0.866 & L 1 \\
\hline 1338 & 10 & 5 & 66 & 8746 & 294 & 45 & $0.2063 E-04$ & $0.1487 E-04$ & 0.721 & LI \\
\hline 1339 & 10 & 5 & 86 & 8766 & 123 & -44 & $0.3724 E-04$ & $0.3765 E-04$ & 1.011 & $\llcorner 1$ \\
\hline 1340 & 10 & 5 & 106 & 8786 & 108 & -46 & $25 E-04$ & $0.4965 z-04$ & 0.950 & L 1 \\
\hline 1341 & 10 & 5 & 126 & 8806 & 108 & -46 & $0.3914 E-04$ & $0.4185 E-04$ & 1.069 & 41 \\
\hline 1343 & 10 & 6 & 6 & 8836 & 76 & -47 & $0.5016 \varepsilon-04$ & $0.4799 E-04$ & 0.957 & L 1 \\
\hline 1345 & 10 & 6 & 46 & 8876 & 64 & -41 & $0.3957 E-04$ & $0.4588 E-04$ & 1.159 & -1 \\
\hline 1347 & 10 & 6 & 86 & 8916 & 62 & -37 & $0.4167 E-04$ & $0.46655-04$ & 1.120 & L 1 \\
\hline 1349 & 11 & 1 & 26 & 9056 & 199 & -54 & $0.6015 E-04$ & $0.5868 E-04$ & 0.976 & 41 \\
\hline 1351 & 11 & 1 & 66 & 9096 & 196 & -55 & $0.5362 E-04$ & $0.5684 E-04$ & 1.060 & L 1 \\
\hline 1353 & 11 & 1 & 106 & 9136 & 196 & -52 & $0.5078 E-04$ & $0.5196 E-04$ & 1.023 & L 1 \\
\hline 1355 & 11 & 1 & 143 & 9176 & 192 & -49 & $0.2599 E-04$ & $0.2794 E-04$ & 1.075 & L 1 \\
\hline 1357 & 11 & 2 & 26 & 9206 & 196 & -51 & $0.2544 E-04$ & $0.25015-04$ & 0.983 & 1 \\
\hline 1359 & 11 & 2 & 66 & 9246 & 198 & -33 & $0.3502 E-04$ & $0.33625-04$ & 0.960 & -1 \\
\hline 1361 & 11 & 2 & 106 & 9286 & 200 & -45 & $0.4665 E-04$ & $15-04$ & 1.066 & L 1 \\
\hline 1363 & 11 & 2 & 146 & 9326 & 203 & -58 & $0.4504 E-04$ & $0.4817 E-04$ & 1.069 & -1 \\
\hline 1364 & 11 & 3 & 6 & 9336 & 225 & -53 & $0.3167 E-04$ & $0.3390 E-04$ & 1.070 & L 1 \\
\hline 1365 & 11 & 3 & 26 & 9356 & 26 & 55 & $0.4916 E-04$ & $0.3987 E-04$ & 0.811 & L 1 \\
\hline 1367 & 11 & 3 & 66 & 9396 & 22 & 47 & $0.4220 E-04$ & $0.3502 E-04$ & 0.830 & L 1 \\
\hline 1369 & 11 & 3 & 106 & 9436 & 17 & 47 & $0.6359 E-04$ & $0.5831 E-04$ & 0.917 & L 1 \\
\hline 1370 & 11 & 3 & 126 & 9456 & 14 & 54 & $0.3733 E-04$ & $0.3120 E-04$ & 0.836 & L 1 \\
\hline 1371 & 11 & 3 & 146 & 9476 & 200 & -29 & $0.6256 E-05$ & $0.7830 E-05$ & 1.252 & $\llcorner 1$ \\
\hline 1373 & 11 & 4 & 26 & 9506 & 205 & -47 & $0.4083 E-04$ & $0.4364 E-04$ & 1.069 & $\llcorner 1$ \\
\hline 1375 & 11 & 4 & 66 & 9546 & 210 & -42 & $0.2594 \subseteq-04$ & $0.2678 E-04$ & 1.033 & L 1 \\
\hline 1377 & 11 & 4 & 106 & 9586 & 208 & -52 & $0.2329 E-04$ & $0.2467 E-04$ & 1.059 & $\lfloor 1$ \\
\hline 1379 & 11 & 4 & 146 & 9626 & 199 & -52 & $0.2965 z-04$ & $0.3123 E-04$ & 1.053 & L 1 \\
\hline 1380 & 11 & 5 & 6 & 9636 & 196 & -57 & $0.8611 E-05$ & $0.1054 E-04$ & 1.223 & L 1 \\
\hline 1381 & 11 & 5 & 26 & 9656 & 42 & 51 & $0.2645 \equiv-04$ & $0.2101 E-04$ & 0.794 & 41 \\
\hline 1383 & 11 & 5 & 66 & 9696 & 42 & 47 & $0.3562 E-04$ & $0.2986 \varepsilon-04$ & 0.838 & $L 1$ \\
\hline 1385 & 11 & 5 & 97 & 9727 & 41 & 49 & $0.4141 E-04$ & $0.3415 E-04$ & 0.825 & L 1 \\
\hline 1386 & 11 & 5 & 116 & 9746 & 85 & 4 & $0.8046 E-05$ & $0.3718 E-05$ & 0.462 & $A 2,4$ \\
\hline 1387 & 11 & 5 & 134 & 9764 & 207 & -48 & $0.4231 E-04$ & $0.42795-04$ & 1.011 & L 1 \\
\hline 1389 & 11 & 6 & 26 & 9806 & 228 & -53 & $0.2945 \varepsilon-04$ & $0.3219 E-04$ & 1.093 & L 1 \\
\hline 1391 & 11 & 6 & 66 & 9846 & 228 & -42 & $0.3202 E-04$ & $0.3241 E-04$ & 1.012 & 11 \\
\hline 1393 & 11 & 6 & 106 & 9886 & 225 & -47 & $0.4696 E-04$ & $0.4246 \vec{E}-04$ & 0.904 & L 1 \\
\hline 1394 & 11 & 6 & 126 & 9906 & 217 & -44 & $0.2680 E-04$ & $0.2554 E-04$ & 0.953 & $\llcorner 1$ \\
\hline 1395 & 11 & 6 & 146 & 9926 & 45 & 56 & $0.3465 E-04$ & $0.2612 E-04$ & 0.754 & L 1 \\
\hline 1397 & 11 & 7 & 21 & 9951 & 44 & 45 & $0.4694 E-04$ & $0.3983 E-04$ & 0.849 & $\llcorner 1$ \\
\hline 1399 & 12 & 1 & 96 & 10013 & 160 & 32 & $0.37095-04$ & $0.3111 \mathrm{E}-04$ & 0.839 & L 1 \\
\hline 1401 & 12 & 1 & 146 & 10054 & 145 & 36 & $0.1900 \mathrm{E}-04$ & $0.1476 E-04$ & 0.777 & L 1 \\
\hline 1402 & 12 & 2 & 4 & 10062 & 169 & 28 & $0.6665 E-05$ & $0.4931 E-05$ & 0.740 & L 1 \\
\hline 1403 & 12 & 2 & 34 & 10092 & 306 & -27 & $0.3246 \tilde{z}-04$ & $0.3797 E-04$ & 1.170 & $\downarrow 1$ \\
\hline 1405 & 12 & 2 & 67 & 10125 & 311 & -22 & $0.8694 E-05$ & $0.7190 E-05$ & 0.827 & A $3-4$ \\
\hline 1407 & 12 & 2 & 106 & 10164 & 314 & -49 & $0.2826 E-04$ & $0.3213 E-04$ & 1.137 & -1 \\
\hline 1408 & 12 & 2 & 126 & 10184 & 142 & 55 & $0.3917 E-04$ & $0.2985 E-04$ & 0.762 & $\llcorner 1$ \\
\hline 1409 & 12 & 2 & 146 & 10204 & 142 & 47 & $0.5624 E-04$ & $0.4447 E-04$ & 0.791 & $\llcorner 1$ \\
\hline 1411 & 12 & 3 & 26 & 10234 & 133 & 49 & $0.4837 E-04$ & $0.4022 E-04$ & 0.832 & $\llcorner 1$ \\
\hline 1413 & 12 & 3 & 66 & 10274 & 136 & 50 & $0.4737 E-04$ & $0.3580 E-04$ & 0.756 & 11 \\
\hline
\end{tabular}


Table 6. (Continued).

\begin{tabular}{|c|c|c|c|c|c|c|c|c|c|c|}
\hline 苞 & ذे & $\begin{array}{l}\stackrel{5}{0} \\
\stackrel{0}{0} \\
\mathscr{W}\end{array}$ & 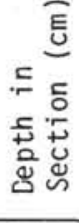 & 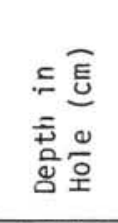 & 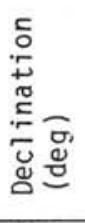 & 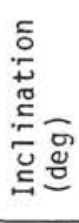 & NRM (emu) & ${ }^{J} 100$ (emu) & ${ }^{3} 100^{/ 3} 0$ & Note \\
\hline 1415 & 12 & 3 & 111 & 10319 & 139 & 52 & $0.4742 E-04$ & $0.3598 E-04$ & 0.759 & L 1 \\
\hline 1416 & 12 & 4 & 6 & 10364 & 145 & 44 & $0.2173 E-04$ & $0.1056 \bar{E}-04$ & 0.485 & L 1 \\
\hline 1417 & 12 & 4 & 26 & 10384 & 313 & -51 & $0.2152 E-04$ & $0.2701 E-04$ & 1.255 & L 1 \\
\hline 1419 & 12 & 4 & 66 & 10424 & 327 & -38 & $0.20765-04$ & $3 E-04$ & 1.163 & L 1 \\
\hline 1421 & 12 & 4 & 106 & 10464 & 307 & -58 & $0.1631 E-04$ & $0.2127 E-04$ & 1.304 & $\llcorner 1$ \\
\hline 1423 & 12 & 4 & 146 & 10504 & 313 & -49 & $0.2302 E-04$ & $2513 E-04$ & 1.092 & $\llcorner 1$ \\
\hline 1425 & 12 & 5 & 26 & 10534 & 310 & -44 & $0.1929 \varepsilon-04$ & $0.22995-04$ & 1.192 & L 1 \\
\hline 1427 & 12 & 5 & 66 & 10574 & 320 & -51 & $0.2865 z-04$ & $0.3227 E-04$ & 1.126 & L 1 \\
\hline 1429 & 12 & 5 & 106 & 10614 & 316 & -31 & $0.12195-04$ & $0.1674 E-04$ & 1.373 & $L 1$ \\
\hline 1431 & 12 & 5 & 146 & 10654 & 307 & -44 & $0.19685-04$ & $0.2228 \varepsilon-04$ & 1.132 & $\llcorner 1$ \\
\hline 1433 & 12 & 6 & 86 & 10744 & 289 & -52 & $0.21398-04$ & $0.2317 E-04$ & 1.083 & L 1 \\
\hline 1435 & 12 & 6 & 123 & 10781 & 274 & -51 & $0.1586 \vec{E}-0.4$ & $0.1749 E-04$ & 1.103 & L 1 \\
\hline 1437 & 13 & 1 & 26 & 10956 & 353 & -49 & $0.8239 E-05$ & $0.1239 E-04$ & 1.503 & L 1 \\
\hline 1438 & 13 & 1 & 46 & 10976 & .159 & 48 & $0.3311 \mathrm{E}-04$ & $0.2597 E-04$ & 0.784 & L 1 \\
\hline 1439 & 13 & 1 & 66 & 10996 & 158 & 49 & $0.3535 E-04$ & $0.2780 E-04$ & 0.786 & L 1 \\
\hline 1440 & 13 & 1 & 86 & 11016 & 164 & 52 & $0.4564 E-04$ & $0.3470 E-04$ & 0.760 & L 1 \\
\hline 1441 & 13 & 1 & 106 & 11036 & 156 & 48 & $0.2597 E-04$ & $0.1777 E-04$ & 0.684 & L 1 \\
\hline 1442 & 13 & 1 & 126 & 11056 & 161 & 40 & $0.3100 E-04$ & $0.2218 E-04$ & 0.715 & L 1 \\
\hline 1443 & 13 & 1 & 146 & 11076 & 169 & 59 & $0.1494 E-04$ & $0.9174 E-05$ & 0.614 & $\llcorner 1$ \\
\hline 1444 & 13 & 2 & 6 & 11086 & 154 & 36 & $0.2060 E-04$ & $0.1534 E-04$ & 0.745 & L 1 \\
\hline 1445 & 13 & 2 & 26 & 11106 & 160 & 47 & $0.30595-04$ & $0.2351 \mathrm{E}-04$ & 0.768 & L 1 \\
\hline 1446 & 13 & 2 & 46 & 11126 & 165 & 45 & $0.3959 \varepsilon-04$ & $0.2761 E-04$ & 0.697 & -1 \\
\hline 1447 & 13 & 2 & 66 & 11146 & 157 & 44 & $0.3294 E-04$ & $0.2547 E-04$ & 0.773 & $\checkmark 1$ \\
\hline 1448 & 13 & 2 & 86 & 11166 & 159 & 46 & $0.3418 E-04$ & $0.2715 E-04$ & 0.794 & $\llcorner 1$ \\
\hline 1449 & 13 & 2 & 106 & 11186 & 172 & -60 & $0.7371 \mathrm{E}-05$ & $0.1523 E-05$ & 0.207 & A $1-3$ \\
\hline 1450 & 13 & 2 & 126 & 11206 & 340 & -51 & $0.1065 E-04$ & $0.1448 E-04$ & 1.359 & -1 \\
\hline 1451 & 13 & 2 & 146 & 11226 & 8 & -49 & $0.1968 E-04$ & $0.2173 E-04$ & 1.104 & L 1 \\
\hline 1452 & 13 & 3 & 6 & 11236 & 340 & -44 & $0.1294 E-04$ & $0.1516 E-04$ & 1.171 & L 1 \\
\hline 1453 & 13 & 3 & 26 & 11256 & 3 & -33 & $0.1418 E-05$ & $0.3569 E-05$ & 2.517 & L 1 \\
\hline 1454 & 13 & 3 & 46 & 11276 & 351 & -44 & $0.1449 E-04$ & $0.1711 \mathrm{E}-04$ & 1.181 & L 1 \\
\hline 1455 & 13 & 3 & 66 & 11296 & 356 & -33 & $0.9721 E-05$ & $0.1608 E-04$ & 1.655 & $\llcorner 1$ \\
\hline 1456 & 13 & 3 & 86 & 11316 & 358 & -47 & $0.1185 E-04$ & $0.1465 E-04$ & 1.237 & 41 \\
\hline 1457 & 13 & 3 & 106 & 11336 & 177 & 32 & $E-04$ & $0.9773 E-05$ & 552 & $\llcorner 1$ \\
\hline 1458 & 13 & 3 & 126 & 11356 & 187 & 44 & $E E-04$ & $0.1979 \varepsilon-04$ & 574 & L 1 \\
\hline 1459 & 13 & 3 & 146 & 11376 & 192 & 47 & $E-04$ & $0.2497 E-04$ & 0.682 & $L 1$ \\
\hline 1460 & 13 & 4 & 6 & 11386 & 204 & 51 & 0. & $E-04$ & 0.721 & L 1 \\
\hline 1461 & 13 & 4 & 26 & 06 & 196 & 45 & 0 . & E- - & 720 & L 1 \\
\hline 1462 & 13 & 4 & 46 & 26 & 162 & -41 & 0 & E- & 790 & 41 \\
\hline 1463 & 13 & 4 & 66 & & 208 & 40 & & 4 & 69 & $\llcorner 1$ \\
\hline 1464 & 13 & 4 & 86 & 11466 & 13 & 15 & $0.4203 E-05$ & $3235 z-05$ & 770 & A $1-3$ \\
\hline 1465 & 13 & 4 & 106 & 11486 & 212 & 47 & $1 E-04$ & $37 E-04$ & 0.671 & 11 \\
\hline 1466 & 13 & 4 & 126 & 11506 & 211 & 45 & $0.2380 E-04$ & $0.1755 E-04$ & 0.737 & L 1 \\
\hline 1467 & 13 & 4 & 146 & 11526 & 213 & 49 & $0.2796 E-04$ & $0.1977 E-04$ & 0.707 & $\llcorner 1$ \\
\hline 1468 & 13 & 5 & 6 & 11536 & 210 & 46 & $0.4190 E-04$ & $0.3183 E-04$ & 0.760 & L 1 \\
\hline 1469 & 13 & 5 & 26 & 11556 & 214 & 49 & $0.2937 E-04$ & $0.2102 E-04$ & 0.716 & $\llcorner 1$ \\
\hline 1470 & 13 & 5 & 46 & 11576 & 212 & 41 & $0.2836 E-04$ & $0.1905 E-04$ & 0.672 & 11 \\
\hline 1471 & 13 & 5 & 66 & 11596 & 56 & 64 & $0.8749 E-05$ & $0.2183 E-05$ & 0.250 & A $2-3$ \\
\hline 1472 & 13 & 5 & 86 & 11616 & 35 & -43 & $0.1354 \mathrm{E}-04$ & $0.1816 E-04$ & 1.341 & L 1 \\
\hline 1473 & 13 & 5 & 106 & 11636 & 30 & -44 & $0.1729 \varepsilon-04$ & $0.2430 E-04$ & 1.405 & 11 \\
\hline 1474 & 13 & 5 & 126 & 11656 & 32 & -45 & $0.1740 E-04$ & $0.2217 E-04$ & 1.274 & L 1 \\
\hline 1475 & 13 & 5 & 146 & 11676 & 24 & -39 & $0.1543 E-04$ & $0.1915 E-04$ & 1.241 & L 1 \\
\hline 1476 & 13 & 6 & 6 & 11686 & 27 & -34 & $0.1514 E-04$ & $0.1852 E-04$ & 1.223 & L 1 \\
\hline 1477 & 13 & 6 & 26 & 11706 & 28 & -41 & $0.1792 E-04$ & $0.2364 E-04$ & 1.319 & L 1 \\
\hline 1478 & 13 & 6 & 46 & 11726 & 30 & -45 & $0.2548 E-04$ & $0.2866 E-04$ & 1.125 & L 1 \\
\hline 1479 & 13 & 6 & 66 & 11746 & 30 & -44 & $0.1807 E-04$ & $0.2376 E-04$ & 1.315 & LI \\
\hline 1480 & 13 & 6 & 86 & 11766 & 30 & -49 & $0.1385 E-04$ & $0.1780 E-04$ & 1.284 & L 1 \\
\hline 1481 & 13 & 6 & 106 & 11786 & 16 & -44 & $0.1404 E-04$ & $0.2264 E-04$ & 1.613 & L 1 \\
\hline 1482 & 13 & 6 & 126 & 11806 & 173 & -10 & $0.25895-05$ & $0.5176 E-05$ & 1.999 & $\llcorner 1$ \\
\hline 1483 & 13 & 6 & 146 & 11826 & 29 & -39 & $0.1924 E-04$ & $0.2846 E-04$ & 1.479 & -1 \\
\hline 1484 & 13 & 7 & 6 & 11836 & 27 & -47 & $0.1312 E-04$ & $0.1708 E-04$ & 1.302 & -1 \\
\hline
\end{tabular}




\begin{tabular}{|c|c|c|c|c|c|c|c|c|c|c|}
\hline$\frac{\frac{\infty}{a}}{\text { 苞 }}$ & ঠั & 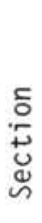 & 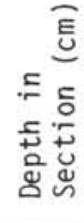 & 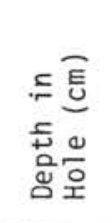 & 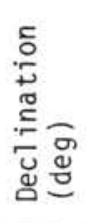 & 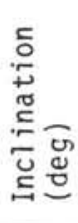 & NRM (emu) & ${ }^{J_{100}}$ (emu) & ${ }^{J_{100}}{ }^{/ J_{0}}$ & Note \\
\hline 1485 & 13 & 7 & 26 & 11856 & 22 & -48 & $0.1200 E-04$ & $0.1936 E-04$ & 1.614 & 11 \\
\hline 1486 & 14 & 1 & 31 & 11866 & 189 & 53 & $0.2533 E-04$ & $0.1723 E-04$ & 0.580 & 41 \\
\hline 1487 & 14 & 1 & 56 & 11891 & 246 & 41 & $0.46245-04$ & $0.3475 E-04$ & 0.752 & 1 \\
\hline 1488 & 14 & 1 & 86 & 11921 & 36 & -60 & $0.8273 E-05$ & $0.1264 E-04$ & 1.528 & 1 \\
\hline 1489 & 14 & 1 & 106 & 11941 & 217 & 46 & $0.4058 E-04$ & $0.2765 E-04$ & 0.681 & L 1 \\
\hline 1490 & 14 & 1 & 146 & 11981 & 37 & -54 & $0.1463 E-05$ & $0.7047 E-05$ & 4.817 & 11 \\
\hline 1491 & 14 & 2 & 6 & 11991 & 39 & -50 & $0.1304 E-04$ & $0.2080 E-04$ & 1.594 & $1-1$ \\
\hline 1492 & 14 & 2 & 26 & 12011 & 33 & -46 & $0.9752 E-05$ & $0.1474 E-04$ & 1.511 & L 1 \\
\hline 1493 & 14 & 2 & 46 & 12031 & 35 & -47 & $0.1231 E-04$ & $0.19185-04$ & 1.558 & 1 \\
\hline 1494 & 14 & 2 & 66 & 12051 & 235 & 50 & $0.2119 \mathrm{E}-04$ & $0.1194 E-04$ & 0.563 & 11 \\
\hline 1495 & 14 & 2 & 86 & 12071 & 207 & 54 & $0.1477 E-04$ & $0.7275 E-05$ & 0.493 & $1-1$ \\
\hline 1496 & 14 & 2 & 106 & 12091 & 31 & -47 & $0.9361 E-05$ & $0.13695-04$ & 1.462 & L 1 \\
\hline 1497 & 14 & 2 & 126 & 12111 & 210 & 50 & $0.2554 \mathrm{E}-04$ & $0.1493 E-04$ & 0.585 & 11 \\
\hline 1498 & 14 & 2 & 146 & 12131 & 208 & 48 & $0.3486 E-04$ & $0.2417 E-04$ & 0.693 & L 1 \\
\hline 1499 & 14 & 3 & 6 & 12141 & 215 & 44 & $0.4112 E-04$ & $0.2663 E-04$ & 0.648 & L 1 \\
\hline 1500 & 14 & 3 & 26 & 12161 & 21 & -45 & $0.3142 E-05$ & $0.8150 E-05$ & 2.594 & L 1 \\
\hline 1501 & 14 & 3 & 46 & 12181 & 214 & 45 & $0.1590 E-04$ & $0.7077 E-05$ & 0.445 & 1 \\
\hline 1502 & 14 & 3 & 66 & 12201 & 216 & 48 & $0.2341 E-04$ & $0.1442 E-04$ & 0.616 & L 1 \\
\hline 1503 & 14 & 3 & 86 & 12221 & 221 & 46 & $0.3661 E-04$ & $0.23735-04$ & 0.648 & 41 \\
\hline 1504 & 14 & 3 & 106 & 12241 & 209 & 48 & $0.5163 E-04$ & $0.4087 E-04$ & 0.792 & L 1 \\
\hline 1505 & 14 & 3 & 126 & 12261 & 218 & 45 & $0.5122 E-04$ & $0.3340 E-04$ & 0.652 & $\square 1$ \\
\hline 1506 & 14 & 3 & 146 & 12281 & 217 & 49 & $0.4075 E-04$ & $0.2651 E-04$ & 0.650 & L 1 \\
\hline 1507 & 14 & 4 & 6 & 12291 & 220 & 40 & $0.4312 E-04$ & $0.3129 E-04$ & 0.726 & 1.1 \\
\hline 1508 & 14 & 4 & 26 & 12311 & 213 & 47 & $0.3900 E-04$ & $0.3051 E-04$ & 0.782 & L 1 \\
\hline 1509 & 14 & 4 & 46 & 12331 & 206 & 47 & $0.3678=-04$ & $0.2183 E-04$ & 0.594 & $\llcorner 1$ \\
\hline 1510 & 14 & 4 & 66 & 12351 & 211 & 37 & $0.3144 E-04$ & $0.2271 E-04$ & 0.722 & L 1 \\
\hline 1511 & 14 & 4 & 86 & 12371 & 210 & 42 & $0.4121 E-04$ & $0.2822 E-04$ & 0.685 & L 1 \\
\hline 1512 & 14 & 4 & 106 & 12391 & 32 & -51 & $0.7912 E-05$ & $0.1485 E-04$ & 1.877 & $\div 1$ \\
\hline 1513 & 14 & 4 & 126 & 12411 & 23 & -47 & $0.6006 E-05$ & $0.6313 E-05$ & 1.051 & $\llcorner 1$ \\
\hline 1514 & 14 & 4 & 146 & 12431 & 8 & -43 & $0.2324 E-04$ & $0.2954 E-05$ & 0.127 & A $2-4$ \\
\hline 1515 & 14 & 5 & 6 & 12441 & 238 & 74 & $0.3502 E-04$ & $0.1038 E-04$ & 0.296 & L 1 \\
\hline 1516 & 14 & 5 & 26 & 12461 & 200 & 42 & $0.3547 E-04$ & $0.2018 E-04$ & 0.569 & L 1 \\
\hline 1517 & 14 & 5 & 46 & 12481 & 24 & -40 & $0.2395 E-04$ & $0.3157 E-04$ & 1.318 & L 1 \\
\hline 1518 & 14 & 5 & 66 & 12501 & 24 & -41 & $0.16985-04$ & $0.2228 E-04$ & 1.312 & -1 \\
\hline 1519 & 14 & 5 & 86 & 12521 & 28 & -41 & $0.2309 E-04$ & $0.3141 E-04$ & 1.360 & L 1 \\
\hline 1520 & 14 & 5 & 106 & 12541 & 21 & -41 & $0.29725-04$ & $0.3448 E-04$ & 1.160 & L. 1 \\
\hline 1521 & 14 & 5 & 126 & 12561 & 21 & -43 & $0.2532 E-04$ & $0.3161 E-04$ & 1.248 & ᄂ 1 \\
\hline 1522 & 14 & 5 & 146 & 12581 & 18 & -46 & $0.2950 E-04$ & $0.35795-04$ & 1.213 & L \\
\hline 1523 & 14 & 6 & 6 & 12591 & 18 & -39 & $0.2763 E-04$ & $0.33405-04$ & 1.209 & ᄂ. 1 \\
\hline 1524 & 14 & 6 & 26 & 12611 & 177 & 46 & $0.1797 E-04$ & $0.9230 \varepsilon-05$ & 0.514 & L 1 \\
\hline 1525 & 14 & 6 & 46 & 12631 & 15 & -35 & $0.1206 E-04$ & $0.2111 E-04$ & 1.750 & L 1 \\
\hline 1526 & 14 & 6 & 66 & 12651 & 197 & 53 & $0.5247 E-04$ & $9 E-04$ & 0.634 & 11 \\
\hline 1527 & 14 & 6 & 86 & 12671 & 201 & 44 & $0.6367 E-04$ & $0.4145 E-04$ & 0.651 & 11 \\
\hline 1528 & 14 & 6 & 106 & 12691 & 200 & 43 & $0.5489 E-04$ & $0.3569 \varepsilon-04$ & 0.650 & 11 \\
\hline 1529 & 14 & 6 & 126 & 12711 & 203 & 45 & $0.48765-04$ & $0.3162 E-04$ & 0.649 & $1-1$ \\
\hline 1530 & 14 & 6 & 138 & 12723 & 197 & 48 & $0.4790 E-04$ & $0.28925-04$ & 0.604 & L 1 \\
\hline 1531 & 14 & 7 & 6 & 12741 & 351 & -26 & $0.9530 E-05$ & $0.4193 E-05$ & 0.440 & 41 \\
\hline 1532 & 14 & 7 & 26 & 12761 & 31 & -38 & $0.1018 E-04$ & $0.2033 E-05$ & 0.200 & 1 \\
\hline 1533 & 14 & 7 & 44 & 12779 & 242 & 47 & $0.1644 \Xi-04$ & $0.2298 E-05$ & 0.140 & L 1 \\
\hline 1538 & 15 & 1 & 44 & 12844 & 10 & 35 & $0.3002 E-04$ & $0.1502 E-04$ & 0.500 & $\llcorner 1$ \\
\hline 1539 & 15 & 1 & 66 & 12866 & 12 & 50 & $0.2763 E-04$ & $0.1818 E-04$ & 0.658 & 41 \\
\hline 1540 & 15 & 1 & 86 & 12886 & 5 & 46 & $0.3703 E-04$ & $0.2156 E-04$ & 0.582 & $1-1$ \\
\hline 1541 & 15 & 1 & 108 & 12908 & 6 & 48 & $0.4506 E-04$ & $0.3042 E-04$ & 0.675 & 11 \\
\hline 1542 & 15 & 1 & 126 & 12926 & 8 & 38 & $0.3070 E-04$ & $0.1880 E-04$ & 0.612 & 11 \\
\hline 1543 & 15 & 1 & 146 & 12946 & 7 & 44 & $0.2548 E-04$ & $0.1646 E-04$ & 0.646 & -1 \\
\hline 1544 & 15 & 2 & 6 & 12956 & 18 & 45 & $0.3706 E-04$ & $0.2395 E-04$ & 0.646 & $\llcorner 1$ \\
\hline 1545 & 15 & 2 & 26 & 12976 & 21 & 40 & $0.35965-04$ & $0.2036 E-04$ & 0.566 & L 1 \\
\hline 1546 & 15 & 2 & 46 & 12996 & 17 & 43 & $0.5665 \varepsilon-04$ & $0.3519 E-04$ & 0.621 & L 1 \\
\hline 1547 & 15 & 2 & 66 & 13015 & 22 & 44 & $0.3195 E-04$ & $0.2053 E-04$ & 0.642 & -1 \\
\hline 1548 & 15 & 2 & 86 & 13036 & 25 & 43 & $0.3378 E-04$ & $0.1933=-04$ & 0.572 & $\div 1$ \\
\hline
\end{tabular}


Table 6. (Continued).

\begin{tabular}{|c|c|c|c|c|c|c|c|c|c|c|}
\hline$\frac{\infty}{\stackrel{0}{E}}$ & ذ্ & 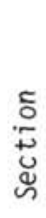 & 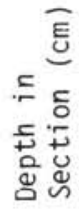 & 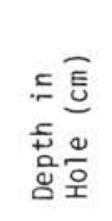 & 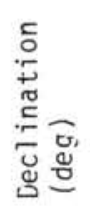 & 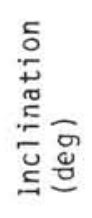 & NRpi (emu) & ${ }^{J_{100}}$ (emu) & ${ }^{\mathrm{J}}{ }_{100} \mathrm{IJ}_{0}$ & Note \\
\hline 1549 & 15 & 2 & 106 & 13056 & 30 & 39 & $0.3428 E-04$ & $0.21875-04$ & 0.638 & $\angle 1$ \\
\hline 1550 & 15 & 2 & 126 & 13076 & 18 & 43 & $0.4259 E-04$ & $0.2585 E-04$ & 0.607 & $L 1$ \\
\hline 1551 & 15 & 2 & 146 & 13096 & 28 & 43 & $0.4667 E-04$ & $0.35795-04$ & 0.767 & 41 \\
\hline 1552 & 15 & 3 & 6 & 13106 & 216 & -40 & $0.9441 E-05$ & $0.1392 E-04$ & 1.474 & 61 \\
\hline 1553 & 15 & 3 & 26 & 13126 & 215 & -45 & $0.3001=-04$ & $0.3488 E-04$ & 1.162 & 41 \\
\hline 1554 & 15 & 3 & 46 & 13146 & 39 & 39 & $0.43765-04$ & $0.3294 E-04$ & 0.753 & L 1 \\
\hline 1555 & 15 & 3 & 66 & 13166 & 224 & -42 & $0.1235 E-04$ & $0.1592 E-04$ & 1.289 & L 1 \\
\hline 1556 & 15 & 3 & 86 & 13186 & 42 & 45 & $0.2121 \mathrm{E}-04$ & $0.1063 E-04$ & 0.501 & 11 \\
\hline 1557 & 15 & 3 & 106 & 13206 & 229 & -38 & $0.49465-05$ & $0.1014 E-04$ & 2.050 & L 1 \\
\hline 1558 & 15 & 3 & 126 & 13226 & 223 & -39 & $0.68965-05$ & $0.1248 E-04$ & 1.809 & 1 \\
\hline 1559 & 15 & 3 & 146 & 13246 & 220 & -47 & $0.2387 E-04$ & $0.28875-04$ & 1.210 & L 1 \\
\hline 1560 & 15 & 4 & 6 & 13256 & 226 & -46 & $0.1605 E-04$ & $0.16575-04$ & 1.032 & 11 \\
\hline 1561 & 15 & 4 & 26 & 13275 & 228 & -46 & $0.1456 E-04$ & $0.1803 E-04$ & 1.239 & L 1 \\
\hline 1562 & 15 & 4 & 46 & 13296 & 54 & 47 & $0.3137 E-04$ & $0.2054 E-04$ & 0.655 & L 1 \\
\hline 1563 & 15 & 4 & 66 & 13316 & 189 & -35 & $0.7430 E-05$ & $0.1533 \subseteq-05$ & 0.206 & L 1 \\
\hline 1564 & 15 & 4 & 86 & 13336 & 246 & -42 & $0.3972 E-05$ & $0.1167 E-04$ & 2.939 & L 1 \\
\hline 1565 & 15 & 4 & 106 & 13356 & 231 & -42 & $0.1475 E-04$ & $0.2004 E-04$ & 1.358 & L 1 \\
\hline 1566 & 15 & 4 & 126 & 13376 & 243 & -36 & $0.8419 E-05$ & $0.1491 E-04$ & 1.771 & L 1 \\
\hline 1567 & 15 & 4 & 146 & 13396 & 236 & -39 & $0.7604 E-05$ & $0.1191 E-04$ & 1.566 & L 1 \\
\hline 1568 & 15 & 5 & 6 & 13406 & 244 & -41 & $0.1656 E-04$ & $0.2228 E-04$ & 1.345 & 41 \\
\hline 1569 & 15 & 5 & 26 & 13426 & 244 & -39 & $0.1789 E-04$ & $0.2333 E-04$ & 1.304 & $\llcorner 1$ \\
\hline 1570 & 15 & 5 & 46 & 13446 & 64 & 47 & $0.2998 \leq-04$ & $0.1630 E-04$ & 0.544 & $1-1$ \\
\hline 1571 & 15 & 5 & 66 & 13466 & 68 & 48 & $0.3657 E-04$ & $0.2274 E-04$ & 0.622 & 41 \\
\hline 1572 & 15 & 5 & 84 & 13484 & 258 & -36 & $0.4662 E-05$ & $0.3502 E-05$ & 0.751 & 11 \\
\hline 1573 & 15 & 5 & 100 & 13500 & 248 & -46 & $0.1232 E-04$ & $0.1737 E-04$ & 1.410 & 41 \\
\hline 1574 & 15 & 5 & 116 & 13516 & 70 & 42 & $0.1740 E-04$ & $0.6583 E-05$ & 0.378 & L 1 \\
\hline 1575 & 15 & 6 & 6 & 13556 & 72 & 43 & $0.4046 E-04$ & $0.2943 E-04$ & 0.727 & L 1 \\
\hline 1576 & 15 & 6 & 26 & 13576 & 109 & 13 & $0.1144 E-04$ & $0.3175 E-05$ & 0.278 & A $2-3$ \\
\hline 1577 & 15 & 6 & 46 & 13596 & 241 & -42 & $0.7020 E-05$ & $0.9208 E-05$ & 1.312 & $\llcorner 1$ \\
\hline 1578 & 15 & 6 & 66 & 13616 & 254 & -48 & $0.1224 E-04$ & $0.1810 E-04$ & 1.479 & ᄂ 1 \\
\hline 1579 & 15 & 6 & 86 & 13636 & 202 & -71 & $0.1065 E-04$ & $0.13995-04$ & 1.314 & 1.1 \\
\hline 1580 & 15 & 6 & 106 & 13656 & 249 & -43 & $0.1101 E-04$ & $0.1888 E-04$ & 1.715 & L 1 \\
\hline 1581 & 15 & 6 & 116 & 13666 & 74 & 51 & $0.2254 E-04$ & $0.1406 E-04$ & 0.624 & $1-1$ \\
\hline 1582 & 15 & 6 & 146 & 13696 & 254 & -30 & $0.7919 E-05$ & $0.1259 E-04$ & 1.591 & $\sqcup 1$ \\
\hline 1583 & 15 & 7 & 6 & 13706 & 250 & -39 & $0.7291 E-05$ & $0.1148 E-04$ & 1.574 & $\leftarrow 1$ \\
\hline 1584 & 15 & 7 & 26 & 13726 & 65 & 43 & $0.3110 E-04$ & $0.1872 E-04$ & 0.602 & 11 \\
\hline 1585 & 15 & 7 & 45 & 13745 & 137 & -72 & $0.8058 E-05$ & $0.4334 E-05$ & 0.538 & $\sqcup 1$ \\
\hline 1586 & 16 & 1 & 36 & 13811 & 175 & -58 & $0.2041 E-04$ & $0.1981 \leq-04$ & 0.971 & L 1 \\
\hline 1587 & 16 & 1 & 56 & 13831 & 180 & -69 & $0.1941 E-04$ & $0.2104 E-04$ & 1.084 & 11 \\
\hline 1588 & 16 & 1 & 76 & 13851 & & & $0.2368 E-04$ & $0.1437 E-04$ & 0.607 & $\mathrm{~F}$ \\
\hline 1589 & 16 & 1 & 96 & 13871 & & & $0.1852 E-04$ & $0.1454 E-04$ & 0.785 & $\mathrm{~F}$ \\
\hline 1590 & 16 & 1 & 116 & 13891 & & & $0.9501 E-05$ & $0.7753 E-06$ & 0.082 & $\mathrm{~F}$ \\
\hline 1591 & 16 & 1 & 136 & 13911 & 127 & -36 & $0.4586 E-05$ & $0.1184 E-04$ & 2.581 & L 1 \\
\hline 1592 & 16 & 2 & 6 & 13931 & 130 & -41 & $0.8434 E-05$ & $0.10755-04$ & 1.274 & L 1 \\
\hline 1593 & 16 & 2 & 26 & 13951 & 310 & 42 & $0.3245 E-04$ & $0.1746 E-04$ & 0.538 & L 1 \\
\hline 1594 & 16 & 2 & 46 & 13971 & 306 & 35 & $0.1881 E-04$ & $0.6758 E-05$ & 0.359 & L 1 \\
\hline 1595 & 15 & 2 & 66 & 13991 & 311 & 47 & $0.2391 E-04$ & $0.1421 E-04$ & 0.595 & L 1 \\
\hline 1596 & 16 & 2 & 86 & 14011 & 116 & -34 & $0.5894 E-05$ & $0.1342 E-04$ & 2.277 & 11 \\
\hline 1597 & 16 & 2 & 106 & 14031 & 115 & -39 & $0.1061 E-05$ & $0.9290 E-05$ & 8.760 & L 1 \\
\hline 1598 & 16 & 2 & 126 & 14051 & 122 & -39 & $0.7173 E-05$ & $0.6423 E-05$ & 0.895 & $\llcorner 1$ \\
\hline 1599 & 16 & 2 & 146 & 14071 & 307 & 38 & $0.2429 E-04$ & $0.1301 E-04$ & 0.536 & L 1 \\
\hline 1600 & 16 & 3 & 6 & 14081 & 292 & 48 & $0.3254 E-04$ & $0.1882 E-04$ & 0.578 & $\llcorner 1$ \\
\hline 1601 & 16 & 3 & 26 & 14101 & 300 & 52 & $0.2697 E-04$ & $0.1707 E-04$ & 0.633 & $\llcorner 1$ \\
\hline 1602 & 16 & 3 & 46 & 14121 & 296 & 44 & $0.3104 E-04$ & $0.1657 E-04$ & 0.534 & L 1 \\
\hline 1603 & 16 & 3 & 66 & 14141 & 299 & 43 & $0.2920 E-0.4$ & $0.1614 E-04$ & 0.553 & L 1 \\
\hline 1604 & 16 & 3 & 86 & 14151 & 299 & 48 & $0.3069 \varepsilon-04$ & $0.16725-04$ & 0.545 & $\downarrow 1$ \\
\hline 1605 & 16 & 3 & 106 & 14181 & 118 & -40 & $0.4671 E-05$ & $0.7214 E-05$ & 1.544 & $\llcorner 1$ \\
\hline 1606 & 16 & 3 & 126 & 14201 & 295 & 54 & $0.2012 E-04$ & $0.7350 E-05$ & 0.365 & $\llcorner 1$ \\
\hline 1607 & 16 & 3 & 146 & 14221 & 304 & 49 & $0.3431 E-04$ & $0.1978 E-04$ & 0.576 & $\llcorner 1$ \\
\hline 1608 & 16 & 4 & 6 & 14231 & 288 & 57 & $0.3891 E-04$ & $0.1945 \equiv-04$ & 0.500 & L i \\
\hline
\end{tabular}


Table 6. (Continued).

\begin{tabular}{|c|c|c|c|c|c|c|c|c|c|c|}
\hline 竞 & ปั & 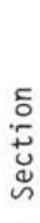 & 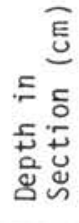 & $\begin{array}{l}\subseteq \text { 틉 } \\
\text { 동 } \\
\text { 응오 }\end{array}$ & 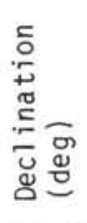 & 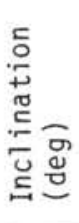 & NRM (emu) & ${ }^{3} 100(\mathrm{emu})$ & ${ }^{3}{ }_{100} /{ }^{3} 0$ & Note \\
\hline 1609 & 16 & 4 & 26 & 14251 & 295 & 49 & $0.2588 E-04$ & $0.1419 E-04$ & 0.548 & $L 1$ \\
\hline 1610 & 16 & 4 & 46 & 14271 & 302 & 52 & $0.3367 E-04$ & $0.1291 E-04$ & 0.383 & $\llcorner 1$ \\
\hline 1611 & 16 & 4 & 66 & 14291 & 304 & 47 & $0.1854 \mathrm{E}-04$ & $0.8913 E-05$ & 0.481 & 1 \\
\hline 1612 & 16 & 4 & 86 & 14311 & 305 & 53 & $0.3482 \varepsilon-04$ & $0.2341 E-04$ & 0.672 & 1.1 \\
\hline 1613 & 16 & 4 & 106 & 14331 & 307 & 55 & $0.24795-04$ & $0.1343 E-04$ & 0.542 & 11 \\
\hline 1614 & 16 & 4 & 126 & 14351 & 113 & -52 & $0.7806 E-05$ & $0.1519 E-04$ & 1.946 & $\div 1$ \\
\hline 1615 & 16 & 4 & 146 & 14371 & 121 & -48 & $0.5035 E-05$ & $0.1095 E-04$ & 2.175 & L 1 \\
\hline 1616 & 16 & 5 & 6 & 14381 & 122 & -39 & $0.3309 E-05$ & $0.78695-05$ & 2.378 & 11 \\
\hline 1617 & 16 & 5 & 26 & 14401 & 108 & -51 & $0.7526 \varepsilon-05$ & $0.13895-04$ & 1.845 & -1 \\
\hline 1618 & 16 & 5 & 46 & 14421 & 297 & 58 & $0.4191 E-04$ & $0.1792 E-04$ & 0.428 & L 1 \\
\hline 1619 & 16 & 5 & 66 & 14441 & 120 & -51 & $0.9065 E-05$ & $0.5156 E-05$ & 0.569 & L \\
\hline 1620 & 16 & 5 & 86 & 14461 & 264 & 53 & $0.3941 E-04$ & $0.1885 E-04$ & 0.478 & L 1 \\
\hline 1621 & 16 & 5 & 106 & 14481 & 295 & 54 & $0.3171 E-04$ & $0.1877 E-04$ & 0.592 & L 1 \\
\hline 1622 & 16 & 5 & 126 & 14501 & 90 & -56 & $0.9104 E-05$ & $0.1775 E-05$ & 0.195 & L 1 \\
\hline 1623 & 16 & 5 & 146 & 14521 & 101 & -56 & $0.3760 E-05$ & $0.1319 E-04$ & 3.507 & L 1 \\
\hline 1624 & 15 & 6 & 7 & 14532 & 97 & -53 & $0.2974 E-05$ & $0.1194 E-04$ & 4.016 & L 1 \\
\hline 1625 & 16 & 6 & 21 & 14546 & 120 & -55 & $O E-05$ & $0.8721 E-05$ & 2.804 & L 1 \\
\hline 1626 & 17 & 1 & 32 & 14734 & 11 & -27 & $0.5588 \varepsilon-05$ & $0.1171 E-04$ & 2.096 & $\llcorner 1$ \\
\hline 1627 & 17 & 1 & 48 & 14750 & 8 & -35 & $0.3507 \varepsilon-05$ & $0.1355 E-04$ & 3.864 & 11 \\
\hline 1628 & 17 & 1 & 67 & 14769 & 358 & -27 & & $0.5876 E-05$ & & -1 \\
\hline 1629 & 17 & 1 & 36 & 14788 & 189 & 41 & $0.2875 E-04$ & $0.8843 E-05$ & 0.308 & L 1 \\
\hline 1630 & 17 & 1 & 106 & 14808 & 188 & 32 & $0.3565 E-04$ & $0.1142 E-04$ & 0.320 & L 1 \\
\hline 1631 & 17 & 1 & 126 & 14828 & 190 & 35 & $0.3124 E-04$ & $0.1310 E-04$ & 0.419 & -1 \\
\hline 1632 & 17 & 1 & 146 & 14848 & 8 & -23 & $0.15195-04$ & $0.3254 E-05$ & 0.214 & L 1 \\
\hline 1633 & 17 & 2 & 11 & 14853 & 28 & -23 & $0.1430 E-04$ & $0.4898 E-05$ & 0.342 & L 1 \\
\hline 1634 & 17 & 2 & 43 & 14895 & 208 & 41 & $0.2591 E-04$ & $0.7429 \varepsilon-05$ & 0.287 & L 1 \\
\hline 1635 & 17 & 2 & 55 & 14907 & 209 & 33 & $0.3334 E-04$ & $0.1251 E-04$ & 0.375 & $\llcorner 1$ \\
\hline 1636 & 17 & 2 & 71 & 14923 & 209 & 34 & $0.2936 E-04$ & $0.7848 E-05$ & 0.267 & $1-1$ \\
\hline 1637 & 17 & 2 & 86 & 14938 & 212 & 31 & $0.3446 E-04$ & $0.1332 E-04$ & 0.386 & L 1 \\
\hline 1638 & 17 & 2 & 106 & 14958 & 216 & 32 & $0.3100 E-04$ & $0.8913 E-05$ & 0.288 & L 1 \\
\hline 1639 & 17 & 2 & 116 & 14978 & 213 & 36 & $0.2674 E-04$ & $0.7604 E-05$ & 0.284 & $L 1$ \\
\hline 1640 & 17 & 2 & 146 & 14998 & 24 & -37 & $0.13045-04$ & $0.4397 E-05$ & 0.337 & $1-1$ \\
\hline 1641 & 17 & 3 & 6 & 15008 & 35 & -4 & $0.1532 E-04$ & $0.2212 E-05$ & 0.144 & L 1 \\
\hline 1642 & 17 & 3 & 26 & 15028 & 36 & -26 & $0.9142 E-05$ & $0.4795 E-05$ & 0.525 & L 1 \\
\hline 1643 & 17 & 3 & 46 & 15048 & 223 & 39 & $0.2501 \mathrm{E}-04$ & $0.7120 E-05$ & 0.285 & L 1 \\
\hline 1644 & 17 & 3 & 66 & 15068 & 224 & 69 & $0.2510 E-04$ & $0.1935=-05$ & 0.077 & $\llcorner 1$ \\
\hline 1645 & 17 & 3 & 82 & 15084 & 230 & 39 & $0.2861 E-04$ & $0.4050 E-05$ & 0.142 & L 1 \\
\hline 1646 & 17 & 3 & 99 & 15101 & 222 & 26 & $0.2749 \varepsilon-04$ & $0.2729 E-05$ & 0.099 & L 1 \\
\hline 1647 & 17 & 3 & 118 & 15120 & 221 & 57 & $0.2796 E-04$ & $0.2341 E-05$ & 0.084 & $\llcorner 1$ \\
\hline 1648 & 17 & 3 & 134 & 15136 & 236 & 68 & $0.2665 E-04$ & $0.2439 E-05$ & 0.092 & $\leq 1$ \\
\hline 1649 & 17 & 4 & 6 & 15158 & & & $0.2442 E-04$ & & & \\
\hline 1650 & 17 & 4 & 26 & 15178 & 80 & 53 & $0.36385-04$ & $0.2573 E-05$ & 0.071 & $\checkmark 1$ \\
\hline 1651 & 17 & 4 & 46 & 15198 & 66 & 38 & $0.3047 E-04$ & $0.4412 E-05$ & 0.145 & 1.1 \\
\hline 1652 & 17 & 4 & 66 & 15218 & 65 & 44 & $0.3240 E-04$ & $0.4173 E-05$ & 0.129 & L 1 \\
\hline 1653 & 17 & 4 & 82 & 15234 & 60 & 40 & $0.4795 E-04$ & $0.9313 E-05$ & 0.194 & L 1 \\
\hline 1654 & 17 & 4 & 100 & 15252 & 52 & 50 & $0.5287 E-04$ & $0.8289 \mathrm{E}-05$ & 0.157 & L 1 \\
\hline 1655 & 17 & 4 & 118 & 15270 & 227 & -53 & $0.2877 E-04$ & $0.4426 E-05$ & 0.154 & L 1 \\
\hline 1656 & 17 & 4 & 136 & 15288 & 65 & 44 & $0.3212 E-04$ & $0.7608 E-05$ & 0.237 & L 1 \\
\hline 1657 & 17 & 5 & 6 & 15308 & 161 & -41 & $0.3230 E-04$ & $0.1493 E-05$ & 0.046 & $\llcorner 1$ \\
\hline 1658 & 17 & 5 & 26 & 15328 & 26 & 43 & $0.4083 E-04$ & $0.5451 E-05$ & 0.134 & L 1 \\
\hline 1659 & 17 & 5 & 46 & 15348 & 170 & -61 & $0.4079 E-04$ & $0.18715-05$ & 0.046 & -1 \\
\hline 1660 & 17 & 5 & 66 & 15368 & 26 & 47 & $0.5119 E-04$ & $0.39995-04$ & 0.781 & $\llcorner 1$ \\
\hline 1661 & 17 & 5 & 86 & 15388 & 77 & -3 & $0.37795-04$ & $0.9297 \mathrm{E}-06$ & 0.025 & $\llcorner 1$ \\
\hline 1662 & 17 & 5 & 106 & 15408 & 32 & 30 & $0.3819 E-04$ & $0.6601 E-05$ & 0.173 & L 1 \\
\hline 1663 & 17 & 5 & 126 & 15428 & 29 & 38 & $0.40745-04$ & $0.6990 E-05$ & 0.172 & -1 \\
\hline 1664 & 18 & 1 & 26 & 15686 & 108 & 19 & $0.3434 E-04$ & $0.1357 E-04$ & 0.395 & 41 \\
\hline 1665 & 18 & 1 & 46 & 15706 & 127 & 30 & $0.3803 E-04$ & $0.16265-04$ & 0.428 & L 1 \\
\hline 1666 & 18 & 1 & 66 & 15726 & 294 & -38 & $0.1403 E-04$ & $0.9060 E-05$ & 0.646 & -1 \\
\hline 1667 & 18 & 1 & 86 & 15746 & 292 & -35 & $0.1273 E-04$ & $0.1168 \mathrm{E}-04$ & 0.918 & L 1 \\
\hline 1668 & 18 & 1 & 106 & 15766 & 69 & -62 & $0.2475 E-04$ & $0.7350 E-06$ & 0.030 & -1 \\
\hline
\end{tabular}


Table 6. (Continued).

\begin{tabular}{|c|c|c|c|c|c|c|c|c|c|c|}
\hline 总 & ปั & 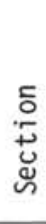 & 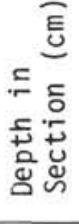 & $\begin{array}{l}\text { 틉 } \\
\text { 픙 } \\
\text { 웜오 }\end{array}$ & 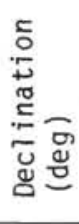 & 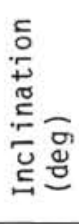 & NRM (emu) & ${ }^{J} 100$ (emu) & ${ }^{3} 100^{/ J_{0}} 0$ & Note \\
\hline 1669 & 18 & 1 & 126 & 15786 & 123 & 56 & $0.3054 E-04$ & $0.3829 E-05$ & 0.125 & $L 1$ \\
\hline 1670 & 18 & 1 & 146 & 15806 & 273 & -32 & $0.2734 E-04$ & $0.1124 E-05$ & 0.041 & L 2 \\
\hline 1671 & 18 & 2 & 6 & 15816 & 109 & 55 & $0.2195 E-04$ & $0.2413 E-05$ & 0.110 & L 1 \\
\hline 1672 & 18 & 2 & 29 & 15839 & 279 & -26 & $0.2490 E-04$ & $0.1746 E-05$ & 0.070 & L 1 \\
\hline 1673 & 18 & 2 & 46 & 15856 & 112 & 44 & $0.4851 E-04$ & $0.1534 E-04$ & 0.316 & 11 \\
\hline 1674 & 18 & 2 & 66 & 15876 & 285 & -39 & $0.1673 E-04$ & $0.9543 E-05$ & 0.570 & $\llcorner 1$ \\
\hline 1675 & 18 & 2 & 86 & 15896 & 280 & -29 & $0.0000 E+00$ & $0.2565 E-04$ & 0.000 & 1 \\
\hline 1676 & 18 & 2 & 106 & 15916 & 284 & -28 & $0.1403 E-04$ & $0.1146 \vec{E}-04$ & 0.816 & L 1 \\
\hline 1677 & 18 & 2 & 126 & 15936 & 290 & -48 & $0.1991 \subseteq-04$ & $0.2438 E-05$ & 0.122 & 11 \\
\hline 1678 & 18 & 2 & 146 & 15956 & 102 & 35 & $0.4185 E-04$ & $0.9439 E-05$ & 0.226 & L 1 \\
\hline 1679 & 18 & 3 & 6 & 15966 & 104 & 29 & $50 E-04$ & $0.2155 E-04$ & 0.427 & L \\
\hline 1680 & 18 & 3 & 26 & 15986 & 95 & 29 & $0.4334 E-04$ & $0.1840 E-04$ & 0.425 & L. 1 \\
\hline 1681 & 18 & 3 & 46 & 16006 & 93 & 43 & $0.3608 E-04$ & $0.1092 E-04$ & 0.303 & L 1 \\
\hline 1682 & 18 & 3 & 66 & 16026 & 101 & 36 & $0.4690 E-04$ & $0.1932 E-04$ & 0.412 & 11 \\
\hline 1683 & 18 & 3 & 86 & 16046 & 267 & 15 & $0.2479 E-04$ & $0.5041 E-05$ & 0.203 & L. 1 \\
\hline 1684 & 18 & 3 & 106 & 16066 & 99 & 26 & $0.40285-04$ & $0.95495-05$ & 0.237 & L 1 \\
\hline 1685 & 18 & 3 & 117 & 16077 & 307 & -46 & $0.2291 E-04$ & $0.2266 E-05$ & 0.099 & L 1 \\
\hline 1686 & 18 & 4 & 6 & 16116 & 293 & -31 & $0.3353 E-04$ & $0.1276 E-05$ & 0.038 & 1.2 \\
\hline 1687 & 18 & 4 & 26 & 16136 & 5 & -61 & $0.3110 E-04$ & $0.3020 E-05$ & 0.097 & 42 \\
\hline 1688 & 18 & 4 & 46 & 16156 & 309 & 1 & $0.2732 E-04$ & $0.1373 E-05$ & 0.050 & A $2-8$ \\
\hline 1689 & 18 & 4 & 66 & 16176 & 260 & -54 & $0.2371 E-04$ & $0.7729 E-05$ & 0.326 & $1-1$ \\
\hline 1690 & 18 & 4 & 86 & 16196 & 107 & 57 & -04 & 0.53 & 0.138 & L 1 \\
\hline 1691 & 18 & 4 & 106 & 16216 & 272 & -11 & $0.2585 E-04$ & $0.17735-05$ & 0.069 & ᄂ 2 \\
\hline 1692 & 18 & 4 & 126 & 16236 & 288 & -15 & $0.4435 E-04$ & $0.1109 E-05$ & 0.025 & $A 2,2,3$ \\
\hline 1693 & 18 & * & 5 & 16248 & 27 & 30 & $0.3709 E-04$ & $0.38475-05$ & 0.104 & L 1 \\
\hline 1694 & 18 & * & 30 & 16273 & 354 & 36 & $0.4899 E-04$ & $0.1875 E-04$ & 0.383 & L 1 \\
\hline 1695 & 19 & 1 & 47 & 16634 & 54 & -38 & $0.2905 E-04$ & $0.1927 E-04$ & 0.663 & L 1 \\
\hline 1696 & 19 & 1 & 66 & 16653 & 196 & -68 & $0.1914 E-04$ & $0.4100 E-05$ & 0.214 & $\llcorner 1$ \\
\hline 1697 & 19 & 1 & 86 & 16673 & 225 & -28 & $0.2365 E-04$ & $0.6389 E-05$ & 0.270 & L 1 \\
\hline 1698 & 19 & 1 & 106 & 16693 & 254 & -18 & $0.3959 E-04$ & $0.1108 E-05$ & 0.028 & 1.2 \\
\hline 1699 & 19 & 1 & 126 & 16713 & 87 & 31 & $0.3263 E-04$ & $0.2728 E-05$ & 0.084 & L 1 \\
\hline 1700 & 19 & 1 & 146 & 16733 & 66 & 38 & $0.5360 E-04$ & $0.1249 \mathrm{E}-04$ & 0.233 & L 1 \\
\hline 1741 & 19 & 2 & 32 & 16769 & 134 & -58 & $0.3322 E-04$ & $0.3195 E-05$ & 0.096 & L 1 \\
\hline 1742 & 19 & 2 & 48 & 16785 & 220 & -24 & $0.3112 E-04$ & $0.1861 E-04$ & 0.598 & L 1 \\
\hline 1743 & 19 & 2 & 66 & 16803 & 61 & 37 & $0.7116 E-04$ & $0.2620 E-04$ & 0.368 & L 1 \\
\hline 1744 & 19 & 2 & 86 & 16823 & 73 & 52 & $0.5539 E-04$ & $0.16185-04$ & 0.292 & 41 \\
\hline 1701 & 19 & 2 & 99 & 16836 & 58 & 38 & $0.6668 E-04$ & $0.2130 E-04$ & 0.319 & $\llcorner 1$ \\
\hline 1702 & 19 & 2 & 111 & 16848 & 236 & -24 & $0.3478 E-04$ & $0.3144 E-05$ & 0.090 & A $2-3$ \\
\hline 1703 & 19 & 2 & 125 & 16862 & 67 & 50 & $0.4658 E-04$ & $0.9889 E-05$ & 0.212 & L 1 \\
\hline 1704 & 19 & 2 & 138 & 16875 & 53 & 40 & $0.6065 E-04$ & $0.1456 E-04$ & 0.240 & L 1 \\
\hline 1705 & 19 & 3 & 6 & 16893 & 65 & 60 & $0.5155 E-04$ & $0.8085 E-05$ & 0.157 & L 1 \\
\hline 1706 & 19 & 3 & 26 & 16913 & 40 & 48 & $0.6502 E-04$ & $3 E-04$ & 288 & L 1 \\
\hline 1707 & 19 & 3 & 46 & 16933 & 40 & 42 & $0.5965 E-04$ & $0.1962 E-04$ & 0.329 & $\downarrow 1$ \\
\hline 1708 & 19 & 3 & 66 & 16953 & 58 & 45 & $0.7369 \mathrm{E}-04$ & $0.1264 E-04$ & 0.172 & L 1 \\
\hline 1709 & 19 & 3 & 86 & 16973 & 36 & 52 & $0.1058 E-03$ & $0.4526 E-04$ & 0.428 & L 1 \\
\hline 1710 & 19 & 3 & 106 & 16993 & 48 & 47 & $0.7406 E-04$ & $0.2157 E-04$ & 0.291 & L 1 \\
\hline 1711 & 19 & * & 5 & 17012 & 131 & 38 & $0.6046 E-04$ & $0.26485-04$ & 0.438 & 11 \\
\hline 1712 & 19 & $*$ & 29 & 17036 & 335 & -51 & $0.7116 E-04$ & $0.2450 E-04$ & 0.344 & L 1 \\
\hline 1534 & 20 & 1 & 6 & 17586 & 21 & -60 & $0.3479 E-04$ & $0.2473 E-04$ & 0.711 & L 1 \\
\hline 1535 & 20 & 1 & 22 & 17602 & 85 & -82 & $0.2995 E-04$ & $0.2995 E-04$ & 1.000 & 11 \\
\hline 1536 & 20 & 1 & 46 & 17626 & 19 & -2 & $0.4952 E-04$ & $0.6246 E-05$ & 0.126 & L 1 \\
\hline 1537 & 20 & 1 & 71 & 17651 & 170 & -59 & $0.3531 \equiv-04$ & $0.7584 E-05$ & 0.215 & L 1 \\
\hline
\end{tabular}

Note: $A=$ vector average of declination and inclination at demagnetization levels $(x 100)$ shown. $L=$ demagnetization level $(x 100)$ used to define declination and in. clination.

$F=$ flow-material (based on shipboard visual descriptions). 
Table 7. Magnetization of Site 578 samples after AF demagnetization at intensities above $100 \mathrm{Oe}$.

\begin{tabular}{|c|c|c|c|c|c|c|c|}
\hline 竞 & ¿ & 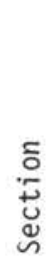 & 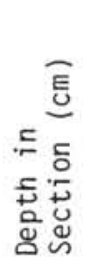 & 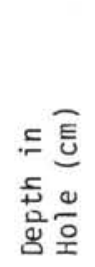 & 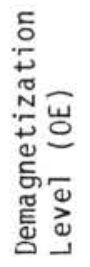 & $J$ (emu) & $\mathrm{J} / \mathrm{J}_{\mathrm{O}}$ \\
\hline 932 & 2 & 1 & 146 & 626 & $\begin{array}{l}200 \\
300 \\
400\end{array}$ & $\begin{array}{l}0.4132 E-04 \\
0.2687 E-04 \\
0.1937 E-04\end{array}$ & $\begin{array}{l}0.695 \\
0.452 \\
0.326\end{array}$ \\
\hline 933 & 2 & 2 & 5 & 635 & $\begin{array}{l}200 \\
300\end{array}$ & $\begin{array}{l}0.2073 E-04 \\
0.1406 E-04\end{array}$ & $\begin{array}{l}0.604 \\
0.410\end{array}$ \\
\hline 934 & 2 & 2 & 26 & 656 & $\begin{array}{l}200 \\
300 \\
400\end{array}$ & $\begin{array}{l}0.3296 E-04 \\
0.2351 E-04 \\
0.1602 E-04\end{array}$ & $\begin{array}{l}0.623 \\
0.444 \\
0.303\end{array}$ \\
\hline 943 & 2 & 3 & 66 & 846 & 200 & $0.3776 E-04$ & 0.615 \\
\hline 970 & 3 & 1 & 106 & 1536 & $\begin{array}{l}200 \\
300 \\
400\end{array}$ & $\begin{array}{l}0.2907 E-04 \\
0.1972 E-04 \\
0.1318 E-04\end{array}$ & $\begin{array}{l}0.604 \\
0.410 \\
0.274\end{array}$ \\
\hline 971 & 3 & 1 & 126 & 1556 & $\begin{array}{l}200 \\
300 \\
400\end{array}$ & $\begin{array}{l}0.2268 E-04 \\
0.1500 E-04 \\
0.1078 E-04\end{array}$ & $\begin{array}{l}0.590 \\
0.391 \\
0.281\end{array}$ \\
\hline 972 & 3 & 1 & 146 & 1576 & $\begin{array}{l}200 \\
300 \\
400\end{array}$ & $\begin{array}{l}0.3206 E-04 \\
0.2194 E-04 \\
0.1430 E-04\end{array}$ & $\begin{array}{l}0.643 \\
0.440 \\
0.287\end{array}$ \\
\hline 980 & 3 & 2 & 146 & 1726 & $\begin{array}{l}200 \\
300 \\
400\end{array}$ & $\begin{array}{l}0.3292 E-04 \\
0.2160 E-04 \\
0.1529 E-04\end{array}$ & $\begin{array}{l}0.564 \\
0.370 \\
0.262\end{array}$ \\
\hline 981 & 3 & 3 & 6 & 1736 & $\begin{array}{l}200 \\
300 \\
400\end{array}$ & $\begin{array}{l}0.3145 E-04 \\
0.2066 E-04 \\
0.1282 E-04\end{array}$ & $\begin{array}{l}0.598 \\
0.393 \\
0.244\end{array}$ \\
\hline 982 & 3 & 3 & 26 & 1756 & $\begin{array}{l}200 \\
300 \\
400\end{array}$ & $\begin{array}{l}0.5557 E-04 \\
0.3565 E-04 \\
0.2460 E-04\end{array}$ & $\begin{array}{l}0.643 \\
0.413 \\
0.285\end{array}$ \\
\hline 1015 & 4 & 1 & 26 & 2406 & $\begin{array}{l}200 \\
300 \\
400\end{array}$ & $\begin{array}{l}0.5280 E-05 \\
0.3119 E-05 \\
0.1905 E-05\end{array}$ & $\begin{array}{l}0.566 \\
0.334 \\
0.204\end{array}$ \\
\hline 1043 & 4 & 3 & 105 & 2785 & $\begin{array}{l}200 \\
300 .\end{array}$ & $\begin{array}{l}0.8119 E-05 \\
0.6588 E-05\end{array}$ & $\begin{array}{l}1.255 \\
1.019\end{array}$ \\
\hline 1033 & 4 & 4 & 65 & 2895 & $\begin{array}{l}200 \\
300 \\
400\end{array}$ & $\begin{array}{l}0.4803 E-06 \\
0.4005 E-06 \\
0.3983 E-06\end{array}$ & $\begin{array}{l}0.931 \\
0.776 \\
0.772\end{array}$ \\
\hline 1035 & 4 & 4 & 110 & 2940 & $\begin{array}{l}200 \\
300 \\
400\end{array}$ & $\begin{array}{l}0.7180 E-06 \\
0.6649 E-06 \\
0.4490 E-06\end{array}$ & $\begin{array}{l}0.757 \\
0.701 \\
0.473\end{array}$ \\
\hline 1057 & 4 & 6 & 66 & 3196 & $\begin{array}{l}200 \\
300\end{array}$ & $\begin{array}{l}0.2376 E-04 \\
0.1644 E-04\end{array}$ & $\begin{array}{l}0.870 \\
0.602\end{array}$ \\
\hline 1062 & 5 & 1 & 106 & 3436 & 200 & $0.8516 E-05$ & 0.441 \\
\hline 107 & 5 & 3 & 128 & 3758 & $\begin{array}{l}200 \\
300 \\
400\end{array}$ & $\begin{array}{l}0.8811 E-05 \\
0.6265 E-05 \\
0.3528 E-05\end{array}$ & $\begin{array}{l}0.404 \\
0.287 \\
0.162\end{array}$ \\
\hline 1080 & 5 & 3 & 146 & 3776 & $\begin{array}{l}200 \\
300 \\
400 \\
401 \\
500 \\
600\end{array}$ & $\begin{array}{l}0.4987 E-05 \\
0.4579 E-05 \\
0.3042 E-05 \\
0.2945 E-05 \\
0.2658 E-05 \\
0.1237 E-05\end{array}$ & $\begin{array}{l}0.123 \\
0.113 \\
0.075 \\
0.073 \\
0.066 \\
0.031\end{array}$ \\
\hline 1107 & 6 & 1 & 6 & 4286 & $\begin{array}{l}200 \\
300\end{array}$ & $\begin{array}{l}0.2013 E-05 \\
0.1515 E-05\end{array}$ & $\begin{array}{l}0.441 \\
0.332\end{array}$ \\
\hline 1129 & 6 & 3 & 146 & 4726 & $\begin{array}{l}200 \\
300\end{array}$ & $\begin{array}{l}0.5970 E-06 \\
0.4529 E-06\end{array}$ & $\begin{array}{l}0.873 \\
0.662\end{array}$ \\
\hline 1160 & 7 & 1 & 146 & 5366 & $\begin{array}{l}200 \\
300\end{array}$ & $\begin{array}{l}0.2766 E-04 \\
0.1873 E-04\end{array}$ & $\begin{array}{l}0.755 \\
0.511\end{array}$ \\
\hline 1161 & 7 & 2 & 6 & 5376 & $\begin{array}{l}200 \\
300\end{array}$ & $\begin{array}{l}0.2213 E-04 \\
0.1601 E-04\end{array}$ & $\begin{array}{l}0.674 \\
0.487\end{array}$ \\
\hline
\end{tabular}


Table 7. (Continued).

\begin{tabular}{|c|c|c|c|c|c|c|c|}
\hline 峁 & ¿ั & 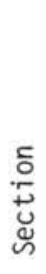 & 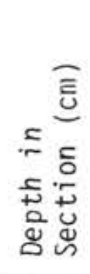 & $\begin{array}{l}=\bar{E} \\
\text { 틍 } \\
\text { 음옹 }\end{array}$ & 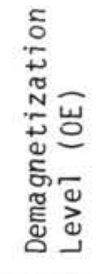 & J (emu) & $\mathrm{J} / \mathrm{J}_{0}$ \\
\hline 1187 & 7 & 5 & 46 & 5866 & 200 & $0.1821 \mathrm{E}-04$ & 1.534 \\
\hline 1203 & 8 & 1 & 26 & 6206 & $\begin{array}{l}200 \\
300 \\
400\end{array}$ & $\begin{array}{l}0.1086 E-04 \\
0.6289 E-05 \\
0.3930 E-05\end{array}$ & $\begin{array}{l}0.722 \\
0.418 \\
0.261\end{array}$ \\
\hline 1204 & 8 & 1 & 46 & 6226 & 200 & $0.9682 E-06$ & 0.868 \\
\hline 1258 & 9 & 1 & 86 & 7216 & $\begin{array}{l}200 \\
300 \\
400 \\
500 \\
600 \\
700 \\
800 \\
900 \\
1000 \\
1001\end{array}$ & $\begin{array}{l}0.4474 E-04 \\
0.3401 E-04 \\
0.2137 E-04 \\
0.1347 E-04 \\
0.9747 E-05 \\
0.6455 E-05 \\
0.4818 E-05 \\
0.3515 E-05 \\
0.1613 E-04 \\
0.2339 E-04\end{array}$ & $\begin{array}{l}0.786 \\
0.598 \\
0.376 \\
0.237 \\
0.171 \\
0.113 \\
0.085 \\
0.062 \\
0.284 \\
0.411\end{array}$ \\
\hline 1261 & 9 & 1 & 146 & 7276 & $\begin{array}{l}200 \\
300 \\
400\end{array}$ & $\begin{array}{l}0.2555 E-04 \\
0.1791 E-04 \\
0.1125 E-04\end{array}$ & $\begin{array}{l}0.578 \\
0.405 \\
0.254\end{array}$ \\
\hline 1277 & 9 & 3 & 146 & 7576 & $\begin{array}{l}200 \\
300 \\
400\end{array}$ & $\begin{array}{l}0.9401 E-05 \\
0.7193 E-05 \\
0.5297 E-05\end{array}$ & $\begin{array}{l}0.595 \\
0.456 \\
0.335\end{array}$ \\
\hline 1281 & 9 & 4 & 66 & 7646 & $\begin{array}{l}200 \\
300 \\
400\end{array}$ & $\begin{array}{l}0.5498 E-04 \\
0.3802 E-04 \\
0.2789 E-04\end{array}$ & $\begin{array}{l}0.720 \\
0.498 \\
0.365\end{array}$ \\
\hline 1303 & 9 & 7 & 6 & 8078 & 200 & $0.9738 E-05$ & 0.824 \\
\hline 1386 & 11 & 4 & 86 & 9746 & $\begin{array}{l}200 \\
400\end{array}$ & $\begin{array}{l}0.1749 E-05 \\
0.5929 E-06\end{array}$ & $\begin{array}{l}0.217 \\
0.074\end{array}$ \\
\hline 1402 & 12 & 2 & 4 & 10062 & 200 & $0.3298 E-05$ & 0.495 \\
\hline 1403 & 12 & 2 & 34 & 10092 & 200 & $0.2829 E-04$ & 0.872 \\
\hline 1405 & 12 & 2 & 67 & 10125 & $\begin{array}{l}200 \\
300 \\
400\end{array}$ & $\begin{array}{l}0.7830 E-05 \\
0.6293 E-05 \\
0.4484 E-05\end{array}$ & $\begin{array}{l}0.901 \\
0.724 \\
0.516\end{array}$ \\
\hline 1449 & 13 & 2 & 106 & 11186 & $\begin{array}{l}200 \\
300\end{array}$ & $\begin{array}{l}0.9654 E-06 \\
0.7596 E-06\end{array}$ & $\begin{array}{l}0.131 \\
0.103\end{array}$ \\
\hline 1453 & 13 & 3 & 6 & 11256 & $\begin{array}{l}200 \\
300\end{array}$ & $\begin{array}{l}0.3595 E-05 \\
0.3129 E-05\end{array}$ & $\begin{array}{l}2.535 \\
2.207\end{array}$ \\
\hline 1462 & 13 & 4 & 46 & 11426 & $\begin{array}{l}200 \\
201 \\
300 \\
400\end{array}$ & $\begin{array}{l}0.2352 E-04 \\
0.2211 E-04 \\
0.1453 E-04 \\
0.1051 E-04\end{array}$ & $\begin{array}{l}0.583 \\
0.548 \\
0.360 \\
0.260\end{array}$ \\
\hline 1464 & 13 & 4 & 86 & 11466 & $\begin{array}{l}200 \\
300 \\
400 \\
401 \\
500 \\
500\end{array}$ & $\begin{array}{l}0.2320 E-05 \\
0.1696 E-05 \\
0.1069 E-05 \\
0.1206 E-05 \\
0.8130 E-06 \\
0.8707 E-06\end{array}$ & $\begin{array}{l}0.552 \\
0.403 \\
0.254 \\
0.287 \\
0.193 \\
0.207\end{array}$ \\
\hline 1471 & 13 & 5 & 6 & 11596 & $\begin{array}{l}200 \\
300\end{array}$ & $\begin{array}{l}0.1755 E-05 \\
0.9173 E-06\end{array}$ & $\begin{array}{l}0.201 \\
0.105\end{array}$ \\
\hline 1488 & 14 & 1 & 86 & 11921 & $\begin{array}{l}200 \\
300\end{array}$ & $\begin{array}{l}0.9574 E-05 \\
0.6095 E-05\end{array}$ & $\begin{array}{l}1.157 \\
0.737 \\
3\end{array}$ \\
\hline $\begin{array}{l}1490 \\
1500\end{array}$ & $\begin{array}{l}14 \\
14\end{array}$ & $\begin{array}{l}1 \\
3\end{array}$ & $\begin{array}{r}146 \\
26\end{array}$ & $\begin{array}{l}11981 \\
12161\end{array}$ & $\begin{array}{l}200 \\
200\end{array}$ & $\begin{array}{l}0.5393 E-05 \\
0.6505 E-05\end{array}$ & $\begin{array}{l}3.686 \\
2.071\end{array}$ \\
\hline 1513 & 14 & 4 & 126 & 12411 & 200 & $0.5417 E-05$ & 0.902 \\
\hline 151 & 14 & 4 & 146 & 12431 & $\begin{array}{l}200 \\
400 \\
401\end{array}$ & $\begin{array}{l}0.2876 E-05 \\
0.2151 E-05 \\
0.1955 E-05\end{array}$ & $\begin{array}{l}0.124 \\
0.093 \\
0.084\end{array}$ \\
\hline 1515 & 14 & 5 & 6 & 12441 & $\begin{array}{l}200 \\
300\end{array}$ & $\begin{array}{l}0.3592 E-05 \\
0.1548 E-05\end{array}$ & $\begin{array}{l}0.103 \\
0.044\end{array}$ \\
\hline
\end{tabular}


Table 7. (Continued).

\begin{tabular}{|c|c|c|c|c|c|c|c|}
\hline 惫 & ป̀ & 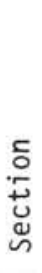 & 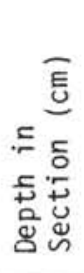 & 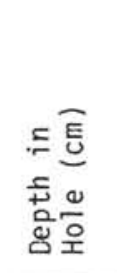 & 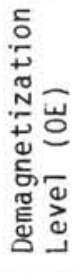 & $J$ (emu) & $\mathrm{J}^{\mathrm{J}} \mathrm{J} 0$ \\
\hline 1524 & 14 & 6 & 26 & 12611 & 200 & $0.4682 E-05$ & .261 \\
\hline 1531 & 14 & 1 & 6 & 741 & 00 & $566 E-05$ & 374 \\
\hline 1532 & 14 & 7 & 26 & 12761 & 200 & $0.2050 E-05$ & .201 \\
\hline 1538 & 15 & 1 & 44 & 12844 & 200 & $0.1030 E-04$ & .343 \\
\hline 1554 & 15 & 3 & 46 & 13146 & 200 & $0.2130 E-04$ & 0.487 \\
\hline 1557 & 15 & 3 & 106 & 13206 & 200 & $0.7991 E-05$ & 1.616 \\
\hline 1563 & 15 & 4 & 66 & 13316 & 200 & $0.2058 E-05$ & 0.277 \\
\hline 1566 & 15 & 4 & 126 & 13376 & 200 & $0.1473 E-04$ & 1.749 \\
\hline 1567 & 15 & 4 & 146 & 13396 & 200 & $0.9878 E-05$ & 1.299 \\
\hline 1572 & 15 & 5 & 84 & 13484 & 200 & $0.2857 E-05$ & 0.613 \\
\hline 1576 & 15 & 6 & 26 & 13576 & $\begin{array}{l}200 \\
300\end{array}$ & $\begin{array}{l}E-05 \\
E-06\end{array}$ & $\begin{array}{l}0.095 \\
0.071\end{array}$ \\
\hline 1577 & 15 & 6 & 46 & 13596 & 200 & 0.7 & 0.999 \\
\hline 1579 & 15 & 6 & 86 & 13636 & $\begin{array}{l}200 \\
300 \\
400\end{array}$ & $\begin{array}{l}0.9886 E-05 \\
0.5618 E-05 \\
0.3977 E-05\end{array}$ & $\begin{array}{l}0.928 \\
0.528 \\
0.373\end{array}$ \\
\hline 1582 & 15 & 6 & 146 & 13696 & 200 & $0.8980 E-05$ & 1.134 \\
\hline & 15 & 7 & 45 & 13745 & $\begin{array}{l}200 \\
300 \\
400 \\
500\end{array}$ & & $\begin{array}{l}0.415 \\
0.276 \\
0.137 \\
0.116\end{array}$ \\
\hline 1586 & 16 & 1 & 36 & 13811 & 200 & 0 . & 0.653 \\
\hline 158 & 15 & 1 & 56 & 13 & $\begin{array}{l}200 \\
300\end{array}$ & $\begin{array}{l}0.14 \\
0.78\end{array}$ & $\begin{array}{l}0.741 \\
0.402\end{array}$ \\
\hline 1590 & 16 & 1 & 116 & 13891 & 200 & 0.35 & 0.037 \\
\hline 159 & 16 & 2 & 6 & 13931 & 200 & 0.85 & 1.014 \\
\hline 1596 & 16 & 2 & 86 & 14011 & 200 & $0.9364 \mathrm{E}-05$ & 1.589 \\
\hline 1597 & 16 & 2 & 106 & 14031 & 200 & 0.7 & 7.340 \\
\hline 1605 & 16 & 3 & 105 & 14181 & 200 & 0 . & 1.079 \\
\hline 1616 & 16 & 5 & 6 & 14381 & 200 & 0 . & 1.854 \\
\hline 1619 & 16 & 5 & 66 & 14441 & 200 & 0.4 & 0.455 \\
\hline 162 & 16 & 5 & 126 & 14501 & $\begin{array}{l}200 \\
300 \\
400\end{array}$ & $\begin{array}{l}0.2322 E-05 \\
0.1419 E-05 \\
0.1134 E-05\end{array}$ & $\begin{array}{l}0.255 \\
0.156 \\
0.125\end{array}$ \\
\hline 162 & 16 & 6 & 7 & 14532 & 200 & $0.7615 E-05$ & 2.560 \\
\hline 162 & 16 & 6 & 21 & 14546 & 200 & $0.6492 E-$ & 2.087 \\
\hline 162 & 17 & 1 & 32 & 14734 & $\begin{array}{l}200 \\
300\end{array}$ & $\begin{array}{l}0.7998 E-05 \\
0.5865 E-05\end{array}$ & $\begin{array}{l}1.431 \\
1.050\end{array}$ \\
\hline 162 & 17 & 1 & 48 & 14750 & 200 & $0.9433 E-05$ & 2.689 \\
\hline 162 & 17 & 1 & 67 & 14769 & 200 & $0.4391 E-05$ & \\
\hline 1632 & 17 & 1 & 146 & 14848 & 200 & $0.3200 E-05$ & 0.211 \\
\hline 163 & 17 & 2 & 6 & 14863 & $\begin{array}{l}200 \\
300\end{array}$ & $\begin{array}{l}0.3618 E-05 \\
0.2515 E-05\end{array}$ & $\begin{array}{l}0.253 \\
0.176\end{array}$ \\
\hline 163 & 1 & 2 & 55 & 18 & 200 & $0.7296 E-05$ & 0.219 \\
\hline 163 & 1 & 2 & 86 & 4938 & 200 & 0. & 0.221 \\
\hline 164 & 1 & 2 & 146 & 14998 & 200 & 0. & 68 \\
\hline 164 & 17 & 3 & 6 & 15008 & 200 & 0. & 0.129 \\
\hline 164 & 1 & 3 & 26 & 15028 & 200 & $0.4+2+3$ & 0.463 \\
\hline 164 & 1 & 3 & 66 & 15068 & 200 & 0. & 19 \\
\hline 1646 & 17 & 3 & 99 & 15101 & 200 & 0.63 & 0.023 \\
\hline 1648 & 17 & 3 & 134 & 15136 & 200 & $0.8466 E-$ & 0 . \\
\hline 164 & 17 & 4 & 6 & 15158 & 200 & 0.80 & 0. \\
\hline 1651 & 17 & 4 & 46 & 15198 & 200 & $0.1333 E-C$ & 0 . \\
\hline 1655 & 17 & 4 & 118 & 15270 & 200 & $0.4578 E-05$ & 0.1 \\
\hline 1656 & 17 & 4 & 136 & 15288 & 200 & $0.3553 E-05$ & 0.11 \\
\hline 165 & 17 & 5 & 6 & 15308 & 200 & $0.2679 E-05$ & 0.083 \\
\hline 165 & 17 & 5 & 46 & 15348 & 200 & $0.3254 E-05$ & 0.080 \\
\hline
\end{tabular}


Table 7. (Continued).

\begin{tabular}{|c|c|c|c|c|c|c|c|}
\hline$\frac{\stackrel{0}{\frac{0}{E}}}{\text { 怘 }}$ & ปั & 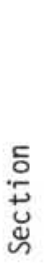 & 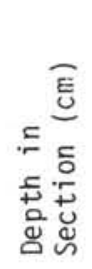 & 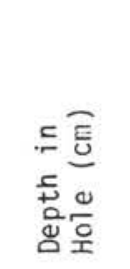 & 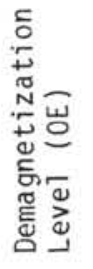 & J (emu) & $\mathrm{J}^{\mathrm{J}} \mathrm{J}_{0}$ \\
\hline 1660 & 17 & 5 & 66 & 15368 & 200 & $0.7063 E-05$ & 0.138 \\
\hline 1661 & 17 & 5 & 86 & 15388 & 200 & $0.1627 E-05$ & 0.043 \\
\hline 1664 & 18 & 1 & 26 & 15686 & $\begin{array}{l}200 \\
300 \\
400\end{array}$ & $\begin{array}{l}0.7207 E-05 \\
0.4591 E-05 \\
0.2686 E-05\end{array}$ & $\begin{array}{l}0.210 \\
0.134 \\
0.078\end{array}$ \\
\hline 1666 & 18 & 1 & 66 & 15726 & 200 & $0.7451 E-05$ & 0.531 \\
\hline 1670 & 18 & 1 & 146 & 15806 & $\begin{array}{l}200 \\
300\end{array}$ & $\begin{array}{l}0.1248 E-05 \\
0.8684 E-06\end{array}$ & $\begin{array}{l}0.046 \\
0.032\end{array}$ \\
\hline 1672 & 18 & 2 & 29 & 15839 & $\begin{array}{l}200 \\
300\end{array}$ & $\begin{array}{l}0.2420 E-05 \\
0.2190 E-05\end{array}$ & $\begin{array}{l}0.097 \\
0.088\end{array}$ \\
\hline 1674 & 18 & 2 & 66 & 15876 & 200 & $0.8124 E-05$ & 0.486 \\
\hline 1675 & 18 & 2 & 86 & 15896 & 200 & $0.1892 E-04$ & 0.000 \\
\hline 1676 & 18 & 2 & 106 & 15916 & 200 & $0.9851 E-05$ & 0.702 \\
\hline 1677 & 18 & 2 & 126 & 15936 & 200 & $0.3407 E-05$ & 0.171 \\
\hline 1680 & 18 & 3 & 26 & 15985 & $\begin{array}{l}200 \\
300\end{array}$ & $\begin{array}{l}0.1291 E-04 \\
0.6404 E-05\end{array}$ & $\begin{array}{l}0.298 \\
0.148\end{array}$ \\
\hline 1682 & 18 & 3 & 66 & 16026 & 200 & $0.1205 E-04$ & 0.257 \\
\hline & 18 & 3 & 86 & 16046 & $\begin{array}{l}200 \\
201 \\
300 \\
400 \\
500 \\
600\end{array}$ & $\begin{array}{l}0.3223 E-05 \\
0.3684 E-05 \\
0.1944 E-05 \\
0.1801 E-05 \\
0.2234 E-05 \\
0.2448 E-05\end{array}$ & $\begin{array}{l}0.130 \\
0.149 \\
0.078 \\
0.073 \\
0.090 \\
0.099\end{array}$ \\
\hline 1684 & 18 & 3 & 106 & 16066 & 200 & $0.4631 E-05$ & 0.115 \\
\hline 1685 & 18 & 3 & 117 & 16077 & 200 & $0.2795 E-05$ & 0.122 \\
\hline 1686 & 18 & 4 & 6 & 16116 & 200 & $0.1562 E-05$ & 0.047 \\
\hline 1687 & 18 & 4 & 26 & 16136 & $\begin{array}{l}200 \\
201\end{array}$ & $\begin{array}{l}0.1360 E-05 \\
0.1098 E-05\end{array}$ & $\begin{array}{l}0.044 \\
0.035\end{array}$ \\
\hline 1688 & 18 & 4 & 46 & 16156 & $\begin{array}{l}200 \\
201 \\
300 \\
400 \\
500 \\
600 \\
700 \\
800\end{array}$ & $\begin{array}{l}0.1122 E-05 \\
0.1096 E-05 \\
0.1169 E-05 \\
0.2011 E-05 \\
0.1770 E-05 \\
0.3131 E-05 \\
0.2435 E-05 \\
0.2655 E-05\end{array}$ & $\begin{array}{l}0.041 \\
0.040 \\
0.043 \\
0.074 \\
0.065 \\
0.115 \\
0.089 \\
0.097\end{array}$ \\
\hline 1689 & 18 & 4 & 66 & 16176 & 200 & $0.5579 E-05$ & 0.235 \\
\hline & 18 & 4 & 86 & 16196 & 200 & 0.2 & 0.069 \\
\hline 169 & 18 & 4 & 106 & 16216 & $\begin{array}{l}200 \\
201 \\
300\end{array}$ & $\begin{array}{l}0.2695 E-05 \\
0.2972 E-05 \\
0.1169 E-05\end{array}$ & $\begin{array}{l}0.104 \\
0.115 \\
0.045\end{array}$ \\
\hline 1692 & 18 & 4 & 126 & 16236 & $\begin{array}{l}200 \\
200\end{array}$ & $\begin{array}{l}0.1328 E-05 \\
0.1290 E-05\end{array}$ & $\begin{array}{l}0.030 \\
0.029\end{array}$ \\
\hline 1693 & 18 & * & 5 & 16248 & $\begin{array}{l}200 \\
201 \\
300\end{array}$ & $\begin{array}{l}0.4618 E-05 \\
0.4476 E-05 \\
0.1837 E-05\end{array}$ & $\begin{array}{l}0.125 \\
0.121 \\
0.050\end{array}$ \\
\hline 1694 & 18 & * & 30 & 16273 & $\begin{array}{l}200 \\
300 \\
400 \\
500 \\
600\end{array}$ & $\begin{array}{l}0.1270 E-04 \\
0.7325 E-05 \\
0.3993 E-05 \\
0.2557 E-05 \\
0.2264 E-05\end{array}$ & $\begin{array}{l}0.259 \\
0.150 \\
0.082 \\
0.052 \\
0.046\end{array}$ \\
\hline 1696 & 19 & 1 & 47 & 16553 & $\begin{array}{l}200 \\
300\end{array}$ & $\begin{array}{l}0.2600 E-05 \\
0.1071 E-05\end{array}$ & $\begin{array}{l}0.136 \\
0.056\end{array}$ \\
\hline 1697 & 19 & 1 & 86 & 16673 & 200 & $0.5269 E-05$ & 0.223 \\
\hline & 19 & 1 & 106 & 16693 & 200 & $0.2138 E-05$ & 0.054 \\
\hline 174 & 19 & 2 & 32 & 16769 & 200 & $0.3776 E-05$ & 0.114 \\
\hline 1743 & 19 & 2 & 66 & 16803 & 200 & $0.1326 E-04$ & 0.186 \\
\hline 1702 & 19 & 2 & 111 & 16848 & 200 & $0.4159 E-05$ & 0.120 \\
\hline
\end{tabular}


Table 7. (Continued).

\begin{tabular}{|c|c|c|c|c|c|c|c|}
\hline 咅 & ปั & 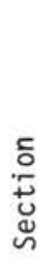 & 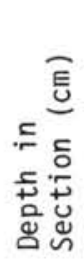 & $\begin{array}{l}\text { 들 } \\
\text { 등 } \\
\text { 음웅 }\end{array}$ & 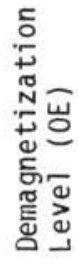 & J (emu) & $\mathrm{J} / \mathrm{J}_{0}$ \\
\hline & & & & & 300 & $0.2160 E-05$ & 0.062 \\
\hline 1534 & 20 & 1 & 6 & 17586 & 200 & $0.1453 E-04$ & 0.418 \\
\hline 1535 & 20 & 1 & 22 & 17602 & $\begin{array}{l}200 \\
300 \\
400 \\
500\end{array}$ & $\begin{array}{l}0.1834 E-04 \\
0.9067 E-05 \\
0.4177 E-05 \\
0.3763 E-05\end{array}$ & $\begin{array}{l}0.612 \\
0.303 \\
0.139 \\
0.126\end{array}$ \\
\hline 1536 & 20 & 1 & 46 & 17626 & $\begin{array}{l}200 \\
300\end{array}$ & $\begin{array}{l}0.2795 E-05 \\
0.1223 E-05\end{array}$ & $\begin{array}{l}0.056 \\
0.025\end{array}$ \\
\hline 1537 & 20 & 1 & 71 & 17651 & 200 & $0.6546 E-05$ & 0.185 \\
\hline
\end{tabular}

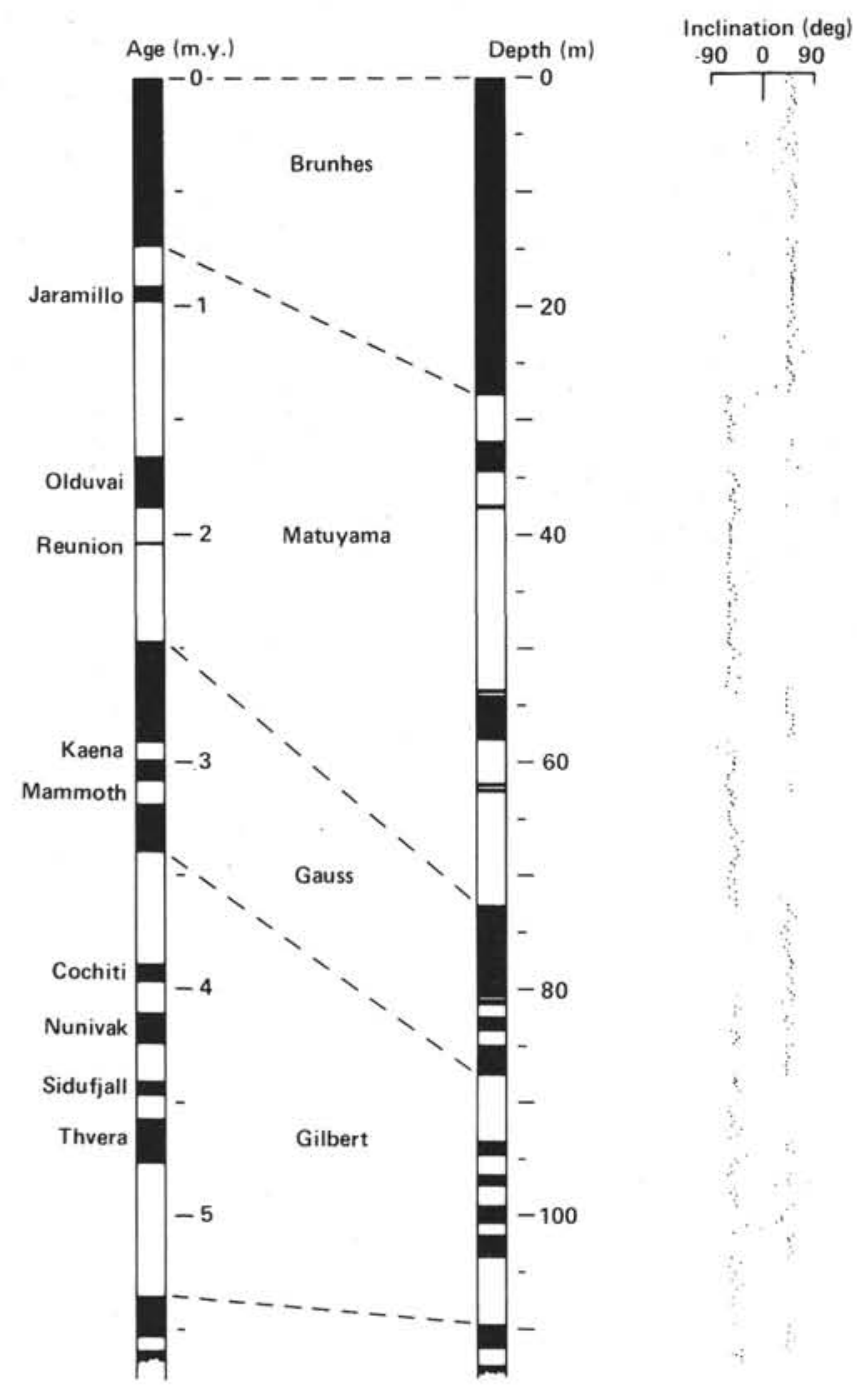

Figure 6. Correlations of the inclination data and paleomagnetic polarity stratigraphy of the upper part of Site 578 with the time scale of Berggren et al. (in press) and the age of the Reunion Event from MacDougall (1977). 
Table 8. Magnetostratigraphy for Hole 578.

\begin{tabular}{|c|c|c|c|}
\hline $\begin{array}{l}\mathrm{Age}^{\mathrm{a}} \\
(\mathrm{m} . \mathrm{y})\end{array}$ & $\begin{array}{l}\text { Depth in hole } \\
\text { (m) }\end{array}$ & Note $^{b}$ & Boundary or event \\
\hline 0.73 & $27.86 \pm 0.19$ & & Brunhes/Matuyama \\
\hline 0.91 & $31.86 \pm 0.09$ & & \\
\hline \multirow[t]{2}{*}{0.98} & $34.46 \pm 0.09$ & & Jaramillo \\
\hline & $\begin{array}{l}37.46 \pm 0.10 \\
37.67 \pm 0.08\end{array}$ & $(+)^{c}$ & \\
\hline \multirow[t]{3}{*}{1.66} & $53.56 \pm 0.09$ & & \\
\hline & $53.07 \pm 0.20$ & $(-)^{c}$ & Olduvai \\
\hline & $54.07 \pm 0.09$ & & Midurar \\
\hline 1.88 & $58.06 \pm 0.09$ & & \\
\hline \multirow[t]{2}{*}{$2.0 \mathrm{i}^{\mathrm{d}}$} & $61.86 \pm 0.19$ & & \\
\hline & $\begin{array}{l}62.16 \pm 0.09 \\
62.36 \pm 0.09\end{array}$ & $(-)^{c}$ & Reunion \\
\hline $2.04^{\mathrm{d}}$ & $62.56 \pm 0.09$ & & \\
\hline \multirow[t]{3}{*}{2.47} & $72.66 \pm 0.09$ & & Matuyama/Gauss \\
\hline & $80.67 \pm 0.10$ & $(-)^{\mathrm{c}}$ & \\
\hline & $80.93 \pm 0.14$ & $(-)^{2}$ & \\
\hline 2.92 & $81.43 \pm 0.15$ & & \\
\hline 2.99 & $82.45 \pm 0.08$ & & Kaena \\
\hline 3.08 & $83.66 \pm 0.09$ & & \\
\hline 3.18 & $84.96 \pm 0.09$ & & Mammoth \\
\hline 3.40 & $87.56 \pm 0.09$ & & Gauss/Gilbert \\
\hline 3.88 & $93.46 \pm 0.09$ & & Cochiti \\
\hline 3.97 & $94.66 \pm 0.09$ & & Cocnin \\
\hline 4.10 & $96.46 \pm 0.09$ & & Nunivak \\
\hline 4.24 & $97.46 \pm 0.18$ & & \\
\hline 4.40 & $99.16 \pm 0.09$ & & Sidufjall \\
\hline 4.47 & $100.77 \pm 0.14$ & & \\
\hline 4.57 & $101.74 \pm 0.09$ & & Thvera \\
\hline 4.77 & $103.74 \pm 0.09$ & & \\
\hline \multirow[t]{2}{*}{5.35} & $109.66 \pm 0.09$ & & $\begin{array}{l}\text { Base of Gilbert } \\
\text { Anomaly } 3.1\end{array}$ \\
\hline & $111.76 \pm 0.09$ & & \\
\hline 5.68 & $113.26 \pm 0.09$ & & \\
\hline 5.89 & $\begin{array}{l}114.16 \pm 0.09 \\
115.96 \pm 0.19\end{array}$ & $(-)^{\mathrm{c}}$ & Anomaly 3.2 \\
\hline \multirow[t]{3}{*}{6.37} & $118.61 \pm 0.04$ & & \\
\hline & $119.06 \pm 0.14$ & $(-)^{\mathrm{c}}$ & Anomaly 3.3 \\
\hline & $119.31 \pm 0.09$ & $(+)$ & \\
\hline 6.50 & $119.61 \pm 0.19$ & & \\
\hline 6.70 & $120.41 \pm 0.09$ & & \\
\hline 6.78 & $120.81 \pm 0.09$ & & \\
\hline \multirow[t]{3}{*}{6.85} & $121.01 \pm 0.09$ & $(-)$ & \\
\hline & $121.51 \pm 0.09$ & $(-)^{\mathrm{c}}$ & Anomaly 4 \\
\hline & $121.71 \pm 0.09$ & & \\
\hline 7.28 & $123.81 \pm 0.09$ & & \\
\hline 7.35 & $124.36 \pm 0.04$ & & \\
\hline 7.41 & $124.71 \pm 0.09$ & & \\
\hline \multirow[t]{2}{*}{7.90} & $126.01 \pm 0.09$ & & \\
\hline & $\begin{array}{l}126.21 \pm 0.09 \\
127.32 \pm 0.08\end{array}$ & $(-)^{\mathrm{c}}$ & Anomaly 4.1 \\
\hline $\begin{array}{l}8.21,8.80 \\
8.92\end{array}$ & & & \\
\hline 10.42 & $\begin{array}{l}127.70 \pm 0.08 \\
131.01 \pm 0.04\end{array}$ & & Anomaly 5 \\
\hline 10.54 & $131.36 \pm 0.09$ & & \\
\hline \multirow[t]{3}{*}{10.59} & $131.56 \pm 0.09$ & $(+)$ & Anomaly $5^{\prime}$ \\
\hline & $131.76 \pm 0.09$ & & \\
\hline & $131.96 \pm 0.09$ & $(+)^{c}$ & \\
\hline 11.03 & $132.86 \pm 0.09$ & $\begin{array}{l}(+) \\
(+)\end{array}$ & \\
\hline 11.09 & $133.06 \pm 0.09$ & & \\
\hline 11.55 & $134.36 \pm 0.09$ & & \\
\hline 11.73 & $134.75 \pm 0.09$ & & \\
\hline 11.86 & $135.08 \pm 0.07$ & & \\
\hline 12.12 & $135.86 \pm 0.09$ & & Anomaly $5 \mathrm{~A}$ \\
\hline 12.46 & $136.61 \pm 0.04$ & $(t)$ & \\
\hline 12.49 & $136.81 \pm 0.14$ & $(+)$ & \\
\hline 12.58 & $137.16 \pm 0.09$ & $(+)$ & \\
\hline 12.62 & $137.36 \pm 0.09$ & $(+)$ & \\
\hline 12.83 & $138.41 \pm 0.09$ & & \\
\hline 13.01 & $139.01 \pm 0.09$ & & \\
\hline 13.20 & $139.41 \pm 0.09$ & & \\
\hline 13.46 & $140.01 \pm 0.09$ & & \\
\hline 13.69 & $140.61 \pm 0.09$ & & \\
\hline 14.08 & $141.71 \pm 0.09$ & $(-)$ & \\
\hline 14.20 & $141.91 \pm 0.09$ & $(-)$ & \\
\hline 14.66 & $143.41 \pm 0.09$ & & \\
\hline 14.87 & $144.11 \pm 0.09$ & & \\
\hline 14.96 & $144.31 \pm 0.09$ & $(-)$ & Anomaly 5B \\
\hline 15.13 & $144.51 \pm 0.09$ & $(-)$ & Anomaly 5B \\
\hline 15.27 & $144.91 \pm 0.09$ & & \\
\hline
\end{tabular}

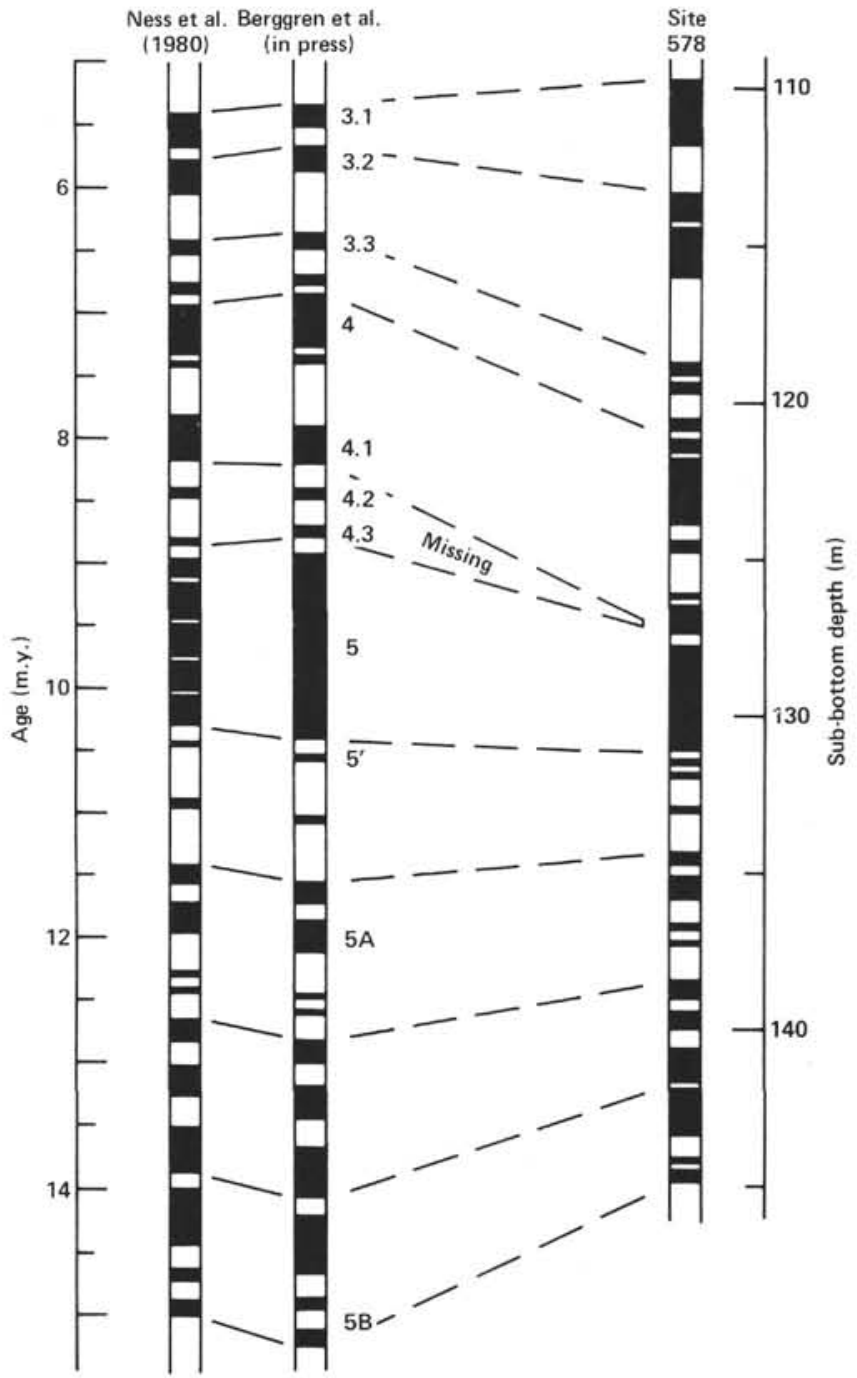

Figure 7. Correlation of the paleomagnetic polarity stratigraphy of the deeper part of Site 578 with the scales of Berggren et al. (in press) and Ness et al. (1980) and marine magnetic Anomalies 3.1 to 5B.

${ }^{a}$ From Berggren et al. (in press).

b $(+)$ or $(-)$ indicate normal or reversed intervals defined by single samples.

c Polarity interval not shown by Berggren et al. (in press).

d Reunion age from MacDougall (1977). 


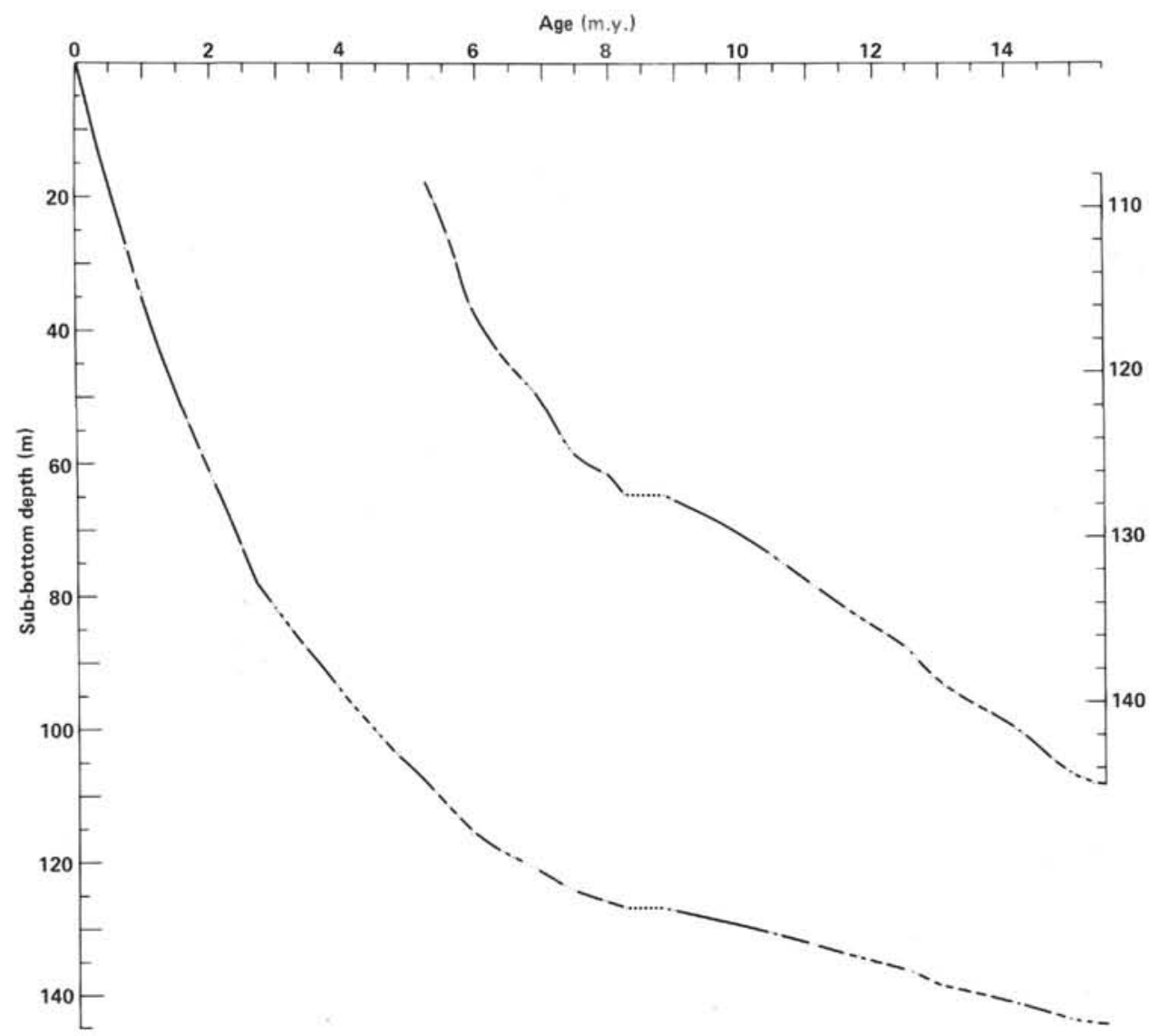

Figure 8. Age-depth curve for Site 578 based on the paleomagnetic correlations of Figures 6 and 7 and the time scale of Berggren et al. (in press). The vertical scale of the 5- to 15-m.y. section (right curve and axis) have been enlarged to show the sedimentation rate changes in more detail.
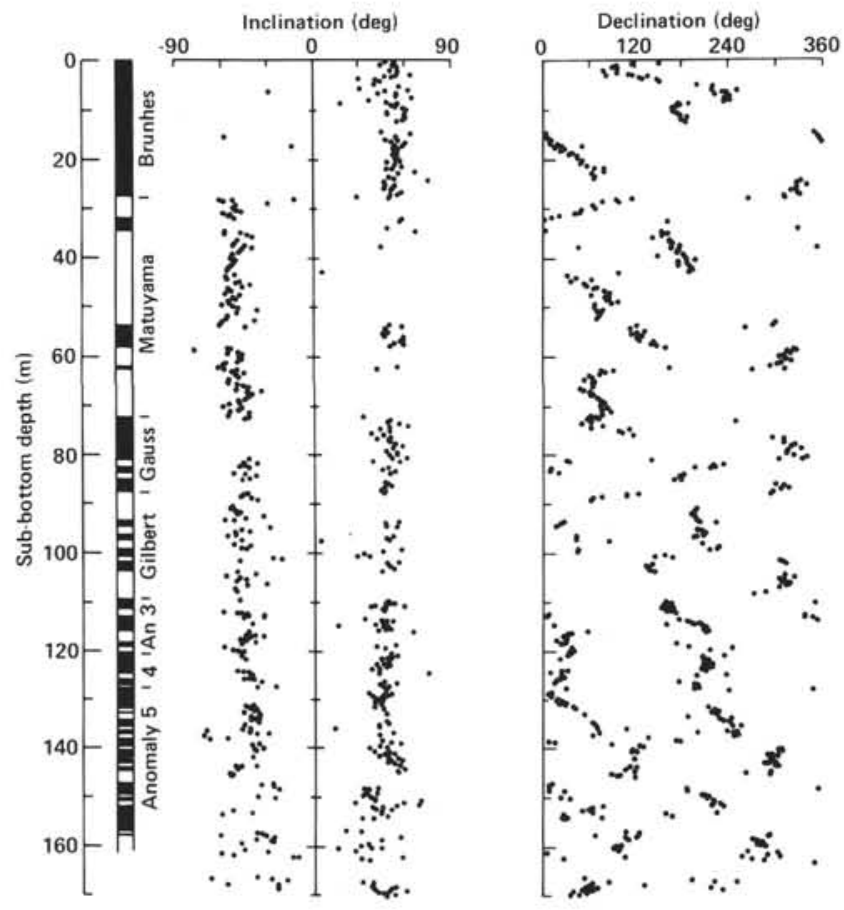

Figure 9. Inclination and declination as a function of depth at Site 578. Rotation of the hydraulic piston core as it cores the sediment is apparent in the declinations for the upper part of the section. 

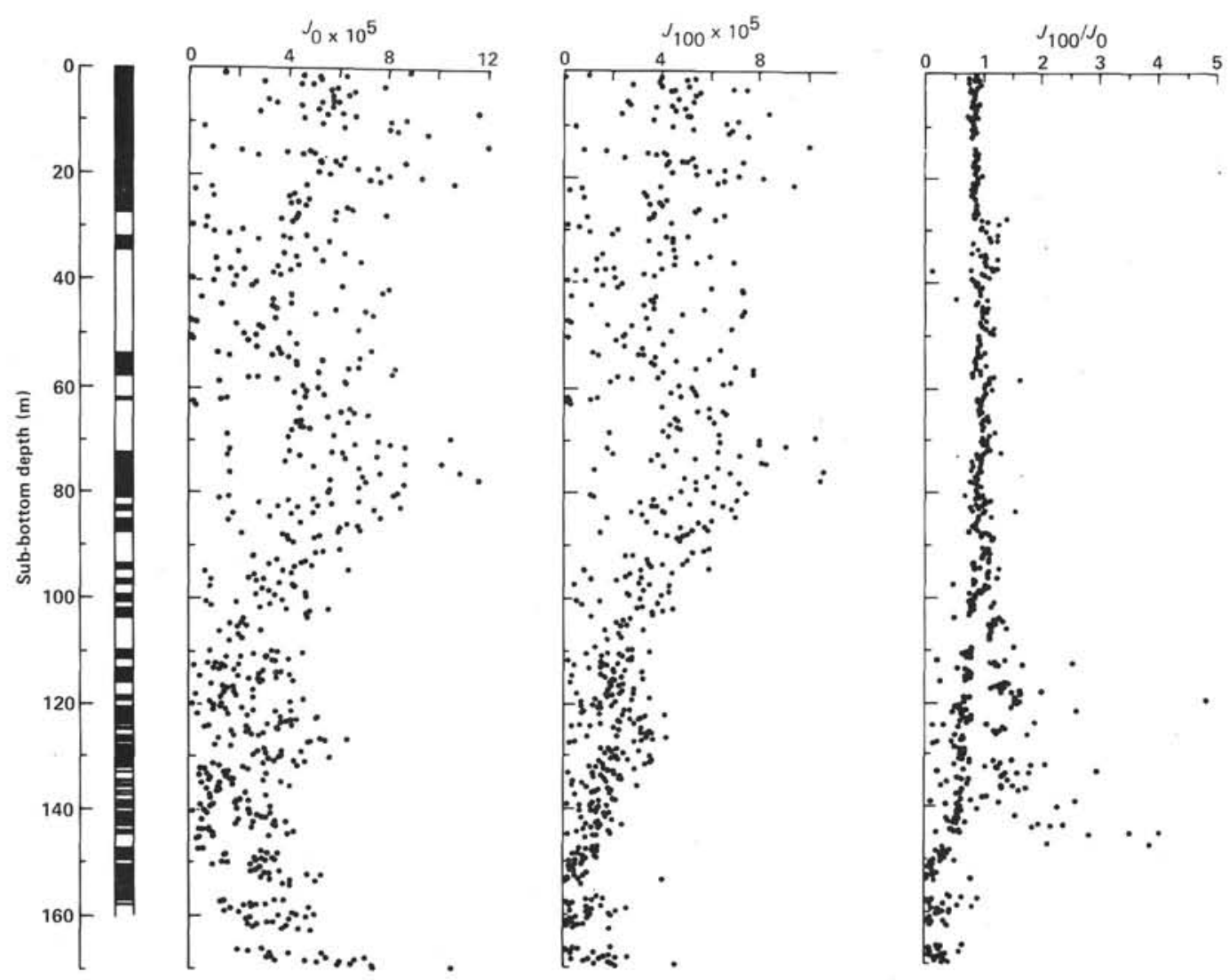

Figure 10. Natural remanent magnetization $\left(J_{0}\right)$, remanent intensity after AF demagnetization at $100 \mathrm{Oe}\left(J_{100}\right)$, and $J_{100} / J_{0}$ as a function of sub-bottom depth at Site 578.

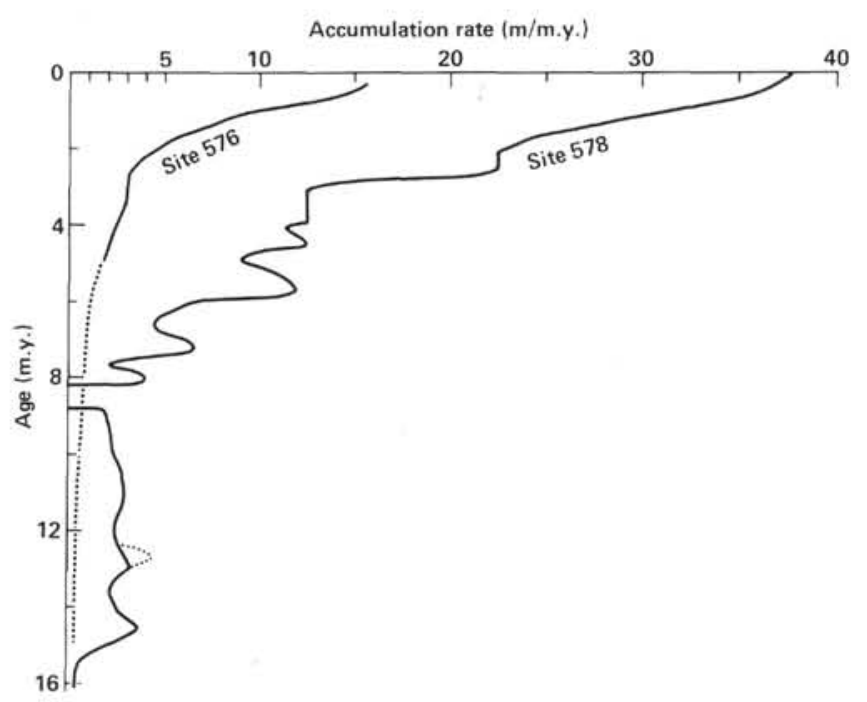

Figure 11. Sediment-accumulation rate as a function of age for the 0to 16-m.y. sections of Sites 576 and 578. These profiles are obtained by differentiating the curves of Figures 2 and 8 against age. Note the similar rates below 16 m.y. and the similar Quaternary rate accelerations at the two sites. The dotted peak at 12.5 m.y. on the Site 578 curve is believed to be an artifact caused by stretching of the sediment section during the coring process. 